\title{
Cobalt-Catalyzed Regio- and Enantioselective Allylic Alkylation of Malononitriles
}

\author{
Samir Ghorai, Sajid Ur Rehman, Wen-Bin Xu, Wen-Yu Huang, Changkun Li*
}

Shanghai Key Laboratory for Molecular Engineering of Chiral Drugs, School of Chemistry and Chemical Engineering, Frontiers Science Center for Transformative Molecules, Shanghai Jiao Tong University, China 200240

E-mail: chkli@sjtu.edu.cn

\section{Context}

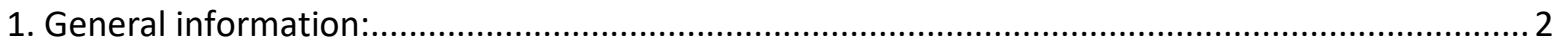

2. Synthesis of (S)-2-(2-bromo-5-methoxyphenyl)-4-methyl-4,5-dihydrooxazole (LS1): ................... 3

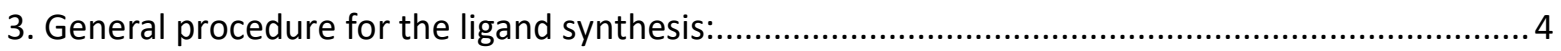

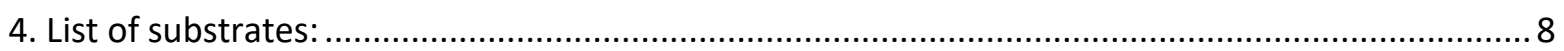

5. Representative procedure of cobalt-catalyzed allylic alkylation of malonitrile: .......................... 9

6. Large scale synthesis of 3aa using 2.5 mol\% catalyst: ............................................................. 9

7. Spectral data of allylic alkylation of malonitrile products: ....................................................... 10

8. Synthesis of (R)-2-(1-phenylallyl)malononitrile (3ja):............................................................ 18

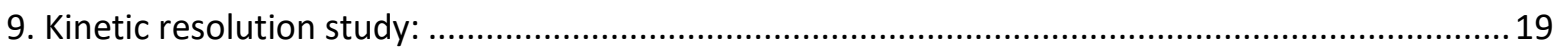

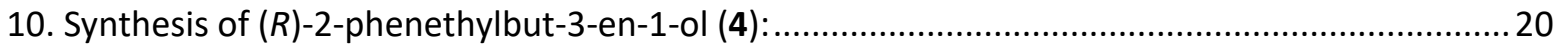

11. Synthesis (R)-2-phenethylcyclopent-3-ene-1,1-dicarbonitrile (5): .........................................21

12. Synthesis $(R)$-2-phenethyl-4-vinylcyclopent-3-ene-1,1-dicarbonitrile (6): ...............................21

13. Synthesis $(R)-5$-(5-phenylpent-1-en-3-yl)pyrimidine-2,4,6-triamine (7):.................................22

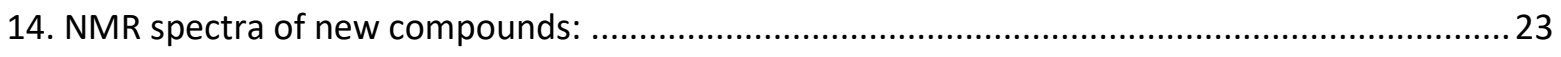

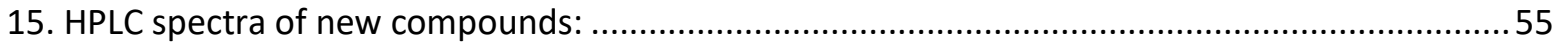

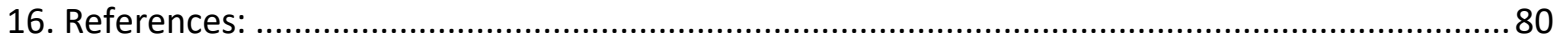




\section{General information:}

All air and moisture sensitive manipulations were carried out with standard Schlenk technique or in a nitrogenfilled glove box. Dried and oxygen free solvents were obtained from solvent purification system (Vigor YJC-7) and used thereafter. Column chromatography was performed on a column of silica gels (200-300 mesh) using either petroleum ether (PE)/ethyl acetate (EA) or dichloromethane (DCM)/MeOH as an eluent. The NMR spectra were recorded on a Bruker-400/500 MHz instrument and chemical shifts are reported in ppm relative to the residual deuterated solvents. High-resolution mass spectra (HRMS) were performed at Instrumental Analysis Center of Shanghai Jiao Tong University with electrospray spectrometer Waters Micromass Q-TOF Premier Mass Spectrometer. Enantiomers ratio were determined by Shimadzu HPLC-16 and Shimadzu HPLC-2030 instrument using DAICEL chiral column at $40{ }^{\circ} \mathrm{C}$. Melting points were measured with Hanon MP100 melting point apparatus. Optical rotations were measured on an Anton Paar MCP100 automatic polarimeter using a 100 $\mathrm{mm}$ path-length cell at $589 \mathrm{~nm}$. $\mathrm{Co}\left(\mathrm{BF}_{4}\right)_{2} \cdot 6 \mathrm{H}_{2} \mathrm{O}$ was purchased from Sigma-Aldrich. All other chemicals were purchased from TCI, Energy Chemical, Macklin or Alfa Aesar. Magnesium monoperoxyphthalate hexahydrate (MMPP) 80\% analytical grade was obtained from Bide Pharmatech, China. Solvents were obtained from tansoole, China. Liquid substrates were distilled and degassed prior to use. 


\section{Synthesis of (S)-2-(2-bromo-5-methoxyphenyl)-4-methyl-4,5-dihydrooxazole (LS1):}

LS1 compound was prepared in two steps as below:

\section{Step 1:}<smiles>COc1ccc(Br)c(C(=O)O)c1</smiles>

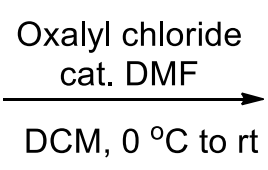<smiles>COc1ccc(Br)c(C(=O)Cl)c1</smiles>

A suspension of 2-bromo-5-methoxybenzoic acid $(4.620 \mathrm{~g}, 20 \mathrm{mmol})$ in DCM (40 mL) was carefully treated with oxalyl chloride $(5.080 \mathrm{~g}, 40 \mathrm{mmol}, 2 \mathrm{eq})$ in the presence of 2 drops of DMF at $0{ }^{\circ} \mathrm{C}$ under inert atmosphere. After stirring at room temperature for 4-5 hours, a clear solution was obtained. The reaction mixture was concentrated under vacuo to get the corresponding benzoyl chloride as light yellowish liquid. The crude product was directly used in the next step.

Step 2:

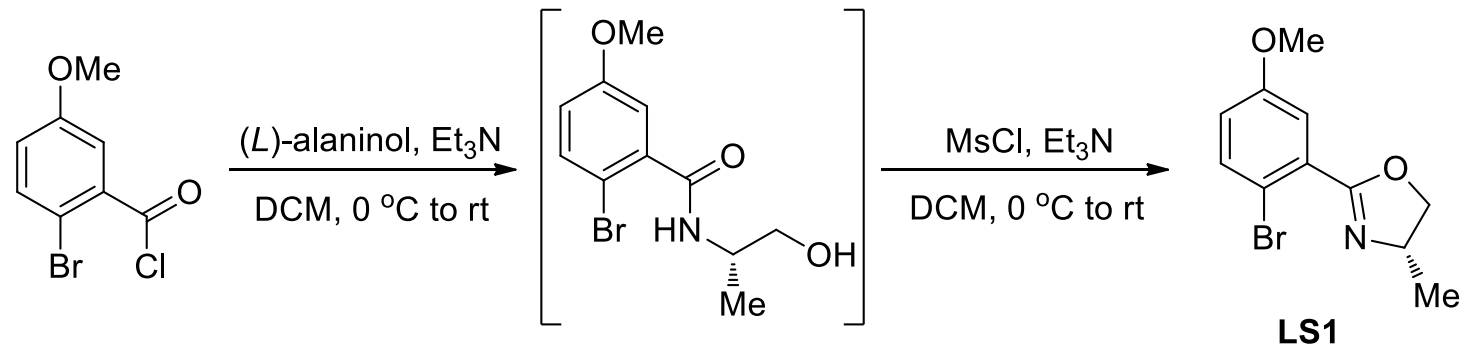

To a solution of $(L)$-alaninol $(1.577 \mathrm{~g}, 21 \mathrm{mmol})$ and $\mathrm{Et}_{3} \mathrm{~N}(8.8 \mathrm{~mL}, 3 \mathrm{eq})$ in DCM (40 mL) was added 2-bromo5-methoxybenzoyl chloride (diluted in $10 \mathrm{~mL}$ of DCM) dropwise at $0{ }^{\circ} \mathrm{C}$. The reaction mixture was stirred at room temperature for 12 hours. After that, it was cooled at $0{ }^{\circ} \mathrm{C}$. Methanesulfonyl chloride $(\mathrm{MsCl})(3.3 \mathrm{~mL}, 2$ eq) and $\mathrm{Et}_{3} \mathrm{~N}(8.8 \mathrm{~mL}, 3 \mathrm{eq})$ were added carefully. The reaction mixture was stirred at room temperature for 4-5 hours. Then, it was washed with water twice and with brine twice. Organic layer was dried by $\mathrm{Na}_{2} \mathrm{SO}_{4}$, and concentrated. The residue was purified by column chromatography (PE/EA, 6:1) to get LS1 as a colorless oil $(4.590 \mathrm{~g}, 85 \%)$.

${ }^{1} \mathbf{H}$ NMR $\left(500 \mathrm{MHz}, \mathrm{CDCl}_{3}\right) \delta 7.47(\mathrm{~d}, J=8.8 \mathrm{~Hz}, 1 \mathrm{H}), 7.20(\mathrm{~d}, J=3.1 \mathrm{~Hz}, 1 \mathrm{H}), 6.82(\mathrm{dd}, J=8.8,3.1 \mathrm{~Hz}, 1 \mathrm{H})$, $4.51(\mathrm{dd}, J=9.4,8.0 \mathrm{~Hz}, 1 \mathrm{H}), 4.39$ (tt, $J=13.4,6.6 \mathrm{~Hz}, 1 \mathrm{H}), 3.97$ (t, $J=7.8 \mathrm{~Hz}, 1 \mathrm{H}), 3.78(\mathrm{~s}, 3 \mathrm{H}), 1.37(\mathrm{~d}, J=$ $6.6 \mathrm{~Hz}, 3 \mathrm{H})$.

${ }^{13}$ C NMR $\left(126 \mathrm{MHz}, \mathrm{CDCl}_{3}\right) \delta 162.9,158.7,134.7,130.7,118.5,116.2,112.3,74.4,62.5,55.8,21.6$.

HRMS (ESI): calculated $m / z$ for $\mathrm{C}_{11} \mathrm{H}_{12} \mathrm{BrNO}_{2}[\mathrm{M}+\mathrm{H}]^{+}=270.0124$, found 270.0131. 


\section{General procedure for the ligand synthesis:}

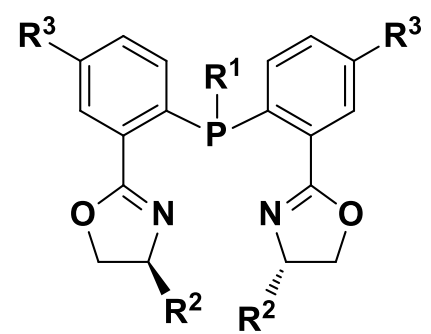

$\mathbf{L 1}\left[\mathrm{R}^{1}=\mathrm{Ph}, \mathrm{R}^{2}=\mathrm{Ph}, \mathrm{R}^{3}=\mathrm{H}\right] ; \mathbf{L} 2\left[\mathrm{R}^{1}=\mathrm{Ph}, \mathrm{R}^{2}={ }^{\mathrm{i}} \mathrm{Pr}, \mathrm{R}^{3}=\mathrm{H}\right] ; \mathbf{L} 3\left[\mathrm{R}^{1}=\mathrm{Ph}, \mathrm{R}^{2}={ }^{\mathrm{t}} \mathrm{Bu}, \mathrm{R}^{3}=\mathrm{H}\right] ; \mathbf{L} 4\left[\mathrm{R}^{1}=\mathrm{Ph}, \mathrm{R}^{2}\right.$ $\left.=\mathrm{Bn}, \mathrm{R}^{3}=\mathrm{H}\right] ; \mathbf{L 5}\left[\mathrm{R}^{1}=\mathrm{Ph}, \mathrm{R}^{2}=\mathrm{Me}, \mathrm{R}^{3}=\mathrm{H}\right]$ were synthesizd according to the reported literature procedure. ${ }^{1}$

L6 $\left[\mathrm{R}^{1}=4-\mathrm{OMe}-3,5-{ }^{\mathrm{t}} \mathrm{Bu}_{2} \mathrm{C}_{6} \mathrm{H}_{2}, \mathrm{R}^{2}=\mathrm{Me}, \mathrm{R}^{3}=\mathrm{H}\right]$, and $\mathbf{L} 7\left[\mathrm{R}^{1}=4-\mathrm{OMe}-3,5-{ }^{\mathrm{t}} \mathrm{Bu}_{2} \mathrm{C}_{6} \mathrm{H}_{2}, \mathrm{R}^{2}=\mathrm{Me}, \mathrm{R}^{3}=\mathrm{OMe}\right]$ were prepared according to the procedure General Method 1, reported in literature. ${ }^{1}$

$\mathbf{L 8}\left[\mathrm{R}^{1}=\mathrm{Me}, \mathrm{R}^{2}=\mathrm{Me}, \mathrm{R}^{3}=\mathrm{H}\right] ; \mathbf{L 9}\left[\mathrm{R}^{1}=\mathrm{Me}, \mathrm{R}^{2}=\mathrm{Me}, \mathrm{R}^{3}=\mathrm{OMe}\right]$ were prepared according to the procedure General Method 2.

\section{General Method 1:}<smiles>[R]c1ccc(Br)c(C2=N[C@@H](C)CO2)c1</smiles>

1. ${ }^{\mathrm{n} B u L i}, \mathrm{THF},-78^{\circ} \mathrm{C}, 1 \mathrm{~h}$ 2. $\mathrm{P}(\mathrm{OPh})_{3} \sim-100^{\circ} \mathrm{C}$ to $\mathrm{rt}, 5 \mathrm{~h}$

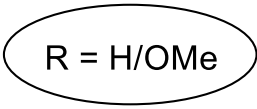<smiles>[R]c1ccc(P(c2ccc([R])cc2C2=NC(C)CO2)c2cc([R])ccc2C2=NC(C)CO2)c(C)c1</smiles>

[Phosphinite solution]<smiles>COc1c(Br)cc(Br)cc1CBr</smiles>

1. ${ }^{\mathrm{n} B u L i}, \mathrm{THF},-78^{\circ} \mathrm{C}, 1 \mathrm{~h}$

2. Phosphinite solution, $-78^{\circ} \mathrm{C}$ to rt, overnight<smiles>[R]c1ccc(C2=NC(C)CO2)c(P(c2ccc(C(C)(C)C)c(OC)c2)c2ccc([R])cc2C(C)(C)C)c1</smiles>

In a flame dried Schlenk tube, 2-bromooxazoline substrate (6 mmol, $2.0 \mathrm{eq})$ was dissolved in THF (12 mL) under an argon atmosphere and the reaction mixture was cooled down to $-78{ }^{\circ} \mathrm{C} .{ }^{n} \mathrm{BuLi}(2.2 \mathrm{eq}, 1.6 \mathrm{M}$ in hexane) was added dropwise and the mixture was stirred for 1 hour at this temperature. The reaction mixture was further 
cooled to $\sim-100{ }^{\circ} \mathrm{C}$ and was added triphenylphosphite $(0.931 \mathrm{~g}, 3 \mathrm{mmol}, 1 \mathrm{eq})$ in $2 \mathrm{~mL}$ of THF in one portion under vigorous stirring. The reaction was slowly warmed up to room temperature and stirred for 5 hours. Phosphinite reaction mixture was used in situ for further substitution to get the desired ligand.

In another flame dried Schlenk tube, lithiation of the 5-bromo-1,3-di-tert-butyl-2-methoxybenzene (1.077 $\mathrm{g}, 3.6 \mathrm{mmol}, 1.2 \mathrm{eq})$ was carried out using ${ }^{\mathrm{n}} \mathrm{BuLi}\left(1.32 \mathrm{eq}, 1.6 \mathrm{M}\right.$ in hexane) at $-78{ }^{\circ} \mathrm{C}$. Then the reaction mixture was added the phosphinite solution via syringe over 10 minutes at $-78{ }^{\circ} \mathrm{C}$. Combined reaction mixture was allowed to warm to room temperature slowly and stirred overnight. The reaction mixture was quenched with water and extracted with ethyl acetate. The solvent was removed and the residue was purified by flash column chromatography on silica gels using PE/EA as an eluent to obtain the pure product.

\section{General Method 2:}
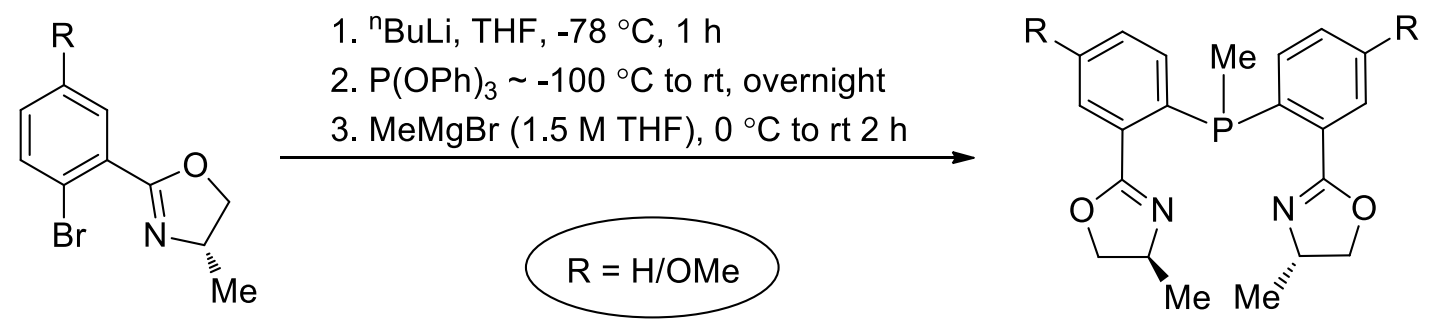

In a flame dried Schlenk tube, 2-bromooxazoline substrate (6 mmol, $2 \mathrm{eq})$ was dissolved in THF (12 mL) under an argon atmosphere and cooled down to $-78{ }^{\circ} \mathrm{C}$. ${ }^{n} \mathrm{BuLi}$ (2.2 eq, $1.6 \mathrm{M}$ in hexane) was added dropwise and the mixture was stirred for 1 hour at this temperature. The reaction mixture was further cooled down to $\sim-100{ }^{\circ} \mathrm{C}$ and was added triphenylphosphite $(0.931 \mathrm{~g}, 3 \mathrm{mmol}, 1 \mathrm{eq})$ in $2 \mathrm{~mL}$ of THF in one portion under vigorous stirring. The reaction was slowly warmed up to room temperature and stirred overnight. Then, $1.5 \mathrm{~mL}$ of $\mathrm{MeMgBr}(3 \mathrm{M}$ in THF) was added dropwise to the reaction mixture at $0{ }^{\circ} \mathrm{C}$. The ice-bath was removed and the reaction mixture was stirred for two hours further at room temperature. The recation mixture was quenced with $2 \mathrm{~mL}$ of degassed water and filtered to remove the solid. Filtrate was dried over $\mathrm{Na}_{2} \mathrm{SO}_{4}$ and solvent was removed under reduced pressure. Crude product was purified by flash column chromatography on silica gels using PE/EA as an eluent to obtain the pure product. 
L6 [R $\left.{ }^{1}=4-O M e-3,5-{ }^{-} \mathrm{Bu}_{2} \mathrm{C}_{6} \mathrm{H}_{2}, \mathbf{R}^{2}=\mathrm{Me}, \mathrm{R}^{3}=\mathrm{H}\right]$ :

White solid; Yield $0.670 \mathrm{~g}, 39 \%$.

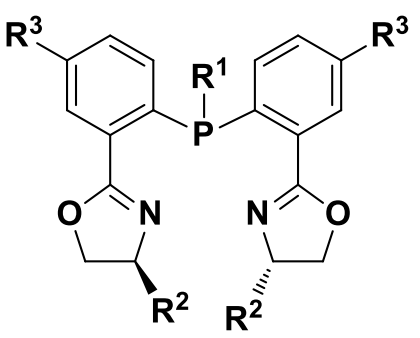

$R^{1}=4-O M e-3,5-{ }^{-} \mathrm{Bu}_{2}-\mathrm{C}_{6} \mathrm{H}_{2}$ $R^{2}=M e ; R^{3}=H$

${ }^{1} \mathbf{H}$ NMR $\left(400 \mathrm{MHz}, \mathrm{CDCl}_{3}\right) \delta 7.82(\mathrm{dd}, J=5.5,3.1 \mathrm{~Hz}, 2 \mathrm{H}), 7.34-7.24(\mathrm{~m}$, $6 \mathrm{H}), 7.01-6.93(\mathrm{~m}, 2 \mathrm{H}), 4.34-4.30(\mathrm{~m}, 1 \mathrm{H}), 4.27-4.15(\mathrm{~m}, 3 \mathrm{H}), 3.75(\mathrm{t}, J=$ $7.6 \mathrm{~Hz}, 1 \mathrm{H}), 3.68$ (s, 3H), $3.64(\mathrm{t}, J=7.7 \mathrm{~Hz}, 1 \mathrm{H}), 1.33(\mathrm{~s}, 18 \mathrm{H}), 1.10(\mathrm{~d}, J=6.5$ $\mathrm{Hz}, 3 \mathrm{H}), 1.06(\mathrm{~d}, J=6.4 \mathrm{~Hz}, 3 \mathrm{H})$.

${ }^{13}$ C NMR $\left(126 \mathrm{MHz}, \mathrm{CDCl}_{3}\right) \delta 164.1(\mathrm{~d}, J=9.5 \mathrm{~Hz}), 160.3(\mathrm{~s}), 143.4(\mathrm{~d}, J=8.0$ $\mathrm{Hz}), 140.6(\mathrm{dd}, J=24.9,14.1 \mathrm{~Hz}), 133.9$ (d, $J=5.8 \mathrm{~Hz}), 133.6(\mathrm{~d}, J=13.0 \mathrm{~Hz})$, 132.4 (dd, $J=82.0,22.0 \mathrm{~Hz}), 130.8(\mathrm{~d}, J=10.6 \mathrm{~Hz}), 130.3(\mathrm{~d}, J=13.7 \mathrm{~Hz})$, 129.9 (dd, $J=16.5,3.7 \mathrm{~Hz}), 127.8(\mathrm{~d}, J=22.0 \mathrm{~Hz}), 73.9$ (d, $J=4.5 \mathrm{~Hz}), 64.4(\mathrm{~s})$, $62.1(\mathrm{~d}, J=18.9 \mathrm{~Hz}), 36.0(\mathrm{~s}), 32.2(\mathrm{~s}), 21.29$ (d, $J=16.9 \mathrm{~Hz})$.

${ }^{31} \mathbf{P}$ NMR $\left(162 \mathrm{MHz}, \mathrm{CDCl}_{3}\right) \delta-5.70$.

$[\boldsymbol{\alpha}]_{\boldsymbol{D}}^{\mathbf{2 5}}=-26.0\left(c 0.25, \mathrm{CHCl}_{3}\right)$.

HRMS (ESI): $m / z[\mathrm{M}+\mathrm{H}]^{+}$Calcd for $\mathrm{C}_{35} \mathrm{H}_{44} \mathrm{~N}_{2} \mathrm{O}_{3} \mathrm{P}$ 571.3084; found 571.3094 .

L7 [ $\mathbf{R}^{1}=$ 4-OMe-3,5-' $\left.{ }^{\mathrm{B}} \mathrm{u}_{2} \mathrm{C}_{6} \mathrm{H}_{2}, \mathbf{R}^{2}=\mathrm{Me}, \mathbf{R}^{3}=\mathrm{OMe}\right]$ :

White solid; Yield $0.700 \mathrm{~g}, 37 \%$.

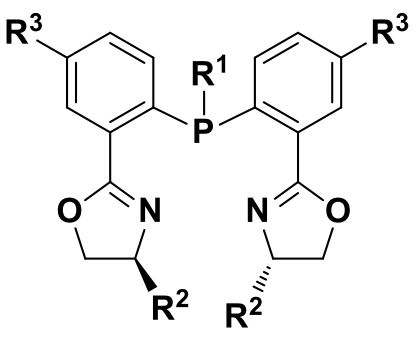

$R^{1}=4-\mathrm{OMe}-3,5-{ }^{\mathrm{t}} \mathrm{Bu}_{2}-\mathrm{C}_{6} \mathrm{H}_{2}$ $R^{2}=M e ; R^{3}=O M e$

${ }^{1} \mathbf{H}$ NMR $\left(500 \mathrm{MHz}, \mathrm{CDCl}_{3}\right) \delta 7.35(\mathrm{dt}, J=8.5,2.6 \mathrm{~Hz}, 2 \mathrm{H}), 7.25(\mathrm{~d}, J=8.0 \mathrm{~Hz}$, 2H), $6.92-6.83(\mathrm{~m}, 4 \mathrm{H}), 4.34(\mathrm{dd}, J=9.2,8.1 \mathrm{~Hz}, 1 \mathrm{H}), 4.27-4.15(\mathrm{~m}, 3 \mathrm{H})$, $3.82(\mathrm{~s}, 6 \mathrm{H}), 3.77(\mathrm{t}, J=7.7 \mathrm{~Hz}, 1 \mathrm{H}), 3.70-3.67(\mathrm{~m}, 4 \mathrm{H}), 1.33(\mathrm{~s}, 18 \mathrm{H}), 1.15(\mathrm{~d}$, $J=6.6 \mathrm{~Hz}, 3 \mathrm{H}), 1.08(\mathrm{~d}, J=6.5 \mathrm{~Hz}, 3 \mathrm{H})$.

${ }^{13}$ C NMR (126 MHz, $\left.\mathrm{CDCl}_{3}\right) \delta 164.4(\mathrm{~s}), 160.2(\mathrm{~s}), 159.3(\mathrm{~d}, J=15.7 \mathrm{~Hz}), 143.4$ (d, $J=7.8 \mathrm{~Hz}), 135.4(\mathrm{~d}, J=44.3 \mathrm{~Hz}), 134.3-133.4(\mathrm{~m}), 133.3(\mathrm{~d}, J=24.1 \mathrm{~Hz})$, $131.8-131.7$ (m), $131.7-131.5(\mathrm{~m}), 116.9$ (d, $J=7.0 \mathrm{~Hz}), 114.7$ (dd, $J=8.3$, $4.4 \mathrm{~Hz}), 74.0(\mathrm{~d}, J=6.8 \mathrm{~Hz}), 64.4(\mathrm{~s}), 62.1(\mathrm{~d}, J=15.6 \mathrm{~Hz}), 55.6(\mathrm{~d}, J=1.8 \mathrm{~Hz})$, 36.0 (s), 32.3 (s), 21.3 (d, $J=21.2 \mathrm{~Hz})$.

${ }^{31} \mathbf{P}$ NMR $\left(202 \mathrm{MHz}, \mathrm{CDCl}_{3}\right) \delta-9.32$.

$[\boldsymbol{\alpha}]_{D}^{25}=-15.6\left(c 0.25, \mathrm{CHCl}_{3}\right)$.

HRMS (ESI): $m / z[\mathrm{M}+\mathrm{H}]^{+}$Calcd for $\mathrm{C}_{37} \mathrm{H}_{48} \mathrm{~N}_{2} \mathrm{O}_{5} \mathrm{P}$ 631.3295; found 631.3307. 
L8 $\left[R^{1}=\right.$ Me, $R^{2}=$ Me, $\left.R^{3}=H\right]:$

White solid; Yield $0.418 \mathrm{~g}, 38 \%$.

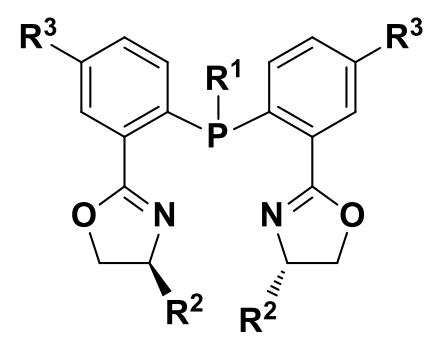

${ }^{1} \mathbf{H}$ NMR $\left(400 \mathrm{MHz}, \mathrm{CDCl}_{3}\right) \delta 7.75-7.71(\mathrm{~m}, 2 \mathrm{H}), 7.38-7.26(\mathrm{~m}, 6 \mathrm{H}), 4.35-$ $4.29(\mathrm{~m}, 2 \mathrm{H}), 4.24-4.13(\mathrm{~m}, 2 \mathrm{H}), 3.82(\mathrm{dd}, J=7.9,7.0 \mathrm{~Hz}, 1 \mathrm{H}), 3.65(\mathrm{t}, J=8.2$ $\mathrm{Hz}, 1 \mathrm{H}), 1.62(\mathrm{~d}, J=5.4 \mathrm{~Hz}, 3 \mathrm{H}), 1.16(\mathrm{dd}, J=9.2,6.6 \mathrm{~Hz}, 6 \mathrm{H})$.

${ }^{13}$ C NMR (101 MHz, $\left.\mathrm{CDCl}_{3}\right) \delta 164.2(\mathrm{~d}, J=16.1 \mathrm{~Hz}), 142.5(\mathrm{t}, J=24.7 \mathrm{~Hz})$, $132.3(\mathrm{dd}, J=40.8,22.3 \mathrm{~Hz}), 131.6(\mathrm{~d}, J=28.4 \mathrm{~Hz}), 130.6(\mathrm{~d}, J=7.9 \mathrm{~Hz}), 129.8$ $\mathbf{R}^{1}=M e ; \mathbf{R}^{2}=M e ; \mathbf{R}^{3}=H$ - $129.7(\mathrm{~m}), 127.8$ (d, $J=15.6 \mathrm{~Hz}), 74.0$ (d, $J=9.5 \mathrm{~Hz}), 62.2$ (d, $J=16.6 \mathrm{~Hz})$, $21.4(\mathrm{~d}, J=13.9 \mathrm{~Hz}), 14.3(\mathrm{~d}, J=17.3 \mathrm{~Hz})$.

${ }^{31} \mathbf{P}$ NMR $\left(162 \mathrm{MHz}, \mathrm{CDCl}_{3}\right) \delta-26.51$.

$[\boldsymbol{\alpha}]_{\boldsymbol{D}}^{\mathbf{2 5}}=-25.2\left(c 0.25, \mathrm{CHCl}_{3}\right)$.

HRMS (ESI): $m / z[\mathrm{M}+\mathrm{H}]^{+}$Calcd for $\mathrm{C}_{21} \mathrm{H}_{24} \mathrm{~N}_{2} \mathrm{O}_{2} \mathrm{P}$ 367.1570; found 367.1575.

L9 $\left[\mathbf{R}^{1}=\mathrm{Me}, \mathbf{R}^{2}=\mathrm{Me}, \mathbf{R}^{3}=\mathrm{OMe}\right]$ :

Colorless sticky oil; Yield $0.460 \mathrm{~g}, 36 \%$.

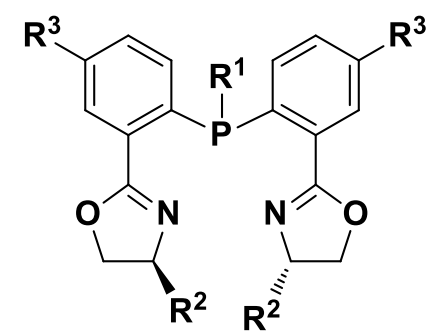

$\mathbf{R}^{1}=\mathrm{Me} ; \mathbf{R}^{2}=\mathrm{Me} ; \mathbf{R}^{3}=\mathrm{OMe}$

${ }^{1} \mathbf{H}$ NMR $\left(400 \mathrm{MHz}, \mathrm{CDCl}_{3}\right) \delta 7.28(\mathrm{dd}, J=5.4,2.7 \mathrm{~Hz}, 2 \mathrm{H}), 7.23-7.17(\mathrm{~m}$, 2H), 6.92 (ddd, $J=8.8,6.3,2.7 \mathrm{~Hz}, 2 \mathrm{H}), 4.39-4.34$ (m, 2H), $4.29-4.17$ (m, $2 \mathrm{H}), 3.85(\mathrm{t}, J=6.0 \mathrm{~Hz}, 1 \mathrm{H}), 3.81(\mathrm{~d}, J=0.6 \mathrm{~Hz}, 6 \mathrm{H}), 3.73(\mathrm{t}, J=8.2 \mathrm{~Hz}, 1 \mathrm{H})$, $1.57(\mathrm{~d}, J=5.5 \mathrm{~Hz}, 3 \mathrm{H}), 1.23(\mathrm{~d}, J=6.6 \mathrm{~Hz}, 3 \mathrm{H}), 1.19(\mathrm{~d}, J=6.6 \mathrm{~Hz}, 3 \mathrm{H})$. ${ }^{13}$ C NMR (101 MHz, $\left.\mathrm{CDCl}_{3}\right) \delta 164.3(\mathrm{~d}, J=10.9 \mathrm{~Hz}), 159.2(\mathrm{~d}, J=10.3 \mathrm{~Hz})$, $134.0-133.2(\mathrm{~m}), 133.2(\mathrm{~s}), 132.9(\mathrm{~s}), 117.0(\mathrm{~d}, J=5.6 \mathrm{~Hz}), 114.6(\mathrm{dd}, J=$ 9.2, $4.7 \mathrm{~Hz}), 74.1(\mathrm{~d}, J=6.6 \mathrm{~Hz}), 62.1(\mathrm{~d}, J=12.1 \mathrm{~Hz}), 55.5(\mathrm{~d}, J=1.5 \mathrm{~Hz})$, $21.4(\mathrm{~d}, J=15.5 \mathrm{~Hz}), 14.5(\mathrm{~d}, J=17.0 \mathrm{~Hz})$.

${ }^{31} \mathbf{P}$ NMR $\left(202 \mathrm{MHz}, \mathrm{CDCl}_{3}\right) \delta-30.91$.

$[\boldsymbol{\alpha}]_{D}^{25}=-6.4\left(c 0.25, \mathrm{CHCl}_{3}\right)$.

HRMS (ESI): $m / z[\mathrm{M}+\mathrm{H}]^{+}$Calcd for $\mathrm{C}_{23} \mathrm{H}_{28} \mathrm{~N}_{2} \mathrm{O}_{4} \mathrm{P}=427.1781$; found 427.1789. 


\section{List of substrates:}<smiles>C=CC(CCc1ccccc1)OC(C)=O</smiles>

[1a]<smiles>C=CC(CC(C)C)OC(C)=O</smiles>

[1e]<smiles>N#CCC#N</smiles>

[2a]<smiles>N#CC(C#N)Cc1ccc2ccccc2c1</smiles>

[2e]<smiles>C=CCC(C#N)C#N</smiles>

[2i]<smiles>COC(C)C=CC(C)OC</smiles>

[1b]<smiles>C=CC(OC(C)=O)C1CC1</smiles>

[1f]<smiles>C=CC(OC(C)=O)c1ccccc1</smiles>

[1i]<smiles>N#CC(C#N)Cc1ccccc1</smiles>

[2b]<smiles>Cn1cccc1CC(C#N)C#N</smiles>

[2f]<smiles>C#CCC(C#N)C#N</smiles>

[2j]<smiles>C=CC(CCCO[Se]C(C)(C)C)OC(C=C)CCCC(CCC)OC(C)=O</smiles>

[1d]<smiles>C=CC(OC(C)=O)C(C)C</smiles>

[1g]<smiles>COOC/C=C/c1ccccc1</smiles>

[1j]<smiles>N#Cc1ccc(CC(C#N)C#N)cc1</smiles>

[2c]<smiles>N#CC(C#N)Cc1ccco1</smiles>

[2g]<smiles>COC(=O)CC#N</smiles>

$\mathrm{PhO}_{2} \mathrm{~S} \smile \mathrm{SO}_{2} \mathrm{Ph}$

All the allylic carbonates were synthesized according to the known literature reported procedure. ${ }^{2} \mathbf{2 a}, 2 \mathbf{2}$ and $\mathbf{2 l}$ were purchased from commercial source directly. Compounds $\mathbf{2 b}$ to $\mathbf{2 h}$ were also prepared accordingly to the known literature procedure. ${ }^{3} \mathbf{2} \mathbf{i}$ and $\mathbf{2} \mathbf{j}$ were known compounds and prepared from malononitrile (2 eq) and corresponding bromide $(1 \mathrm{eq})$ in the presence of Hünig's base $(1 \mathrm{eq})$ in dichloromethane $(0.2 \mathrm{M})$ solvent. ${ }^{4}$ 


\section{Representative procedure of cobalt-catalyzed allylic alkylation of malonitrile:}

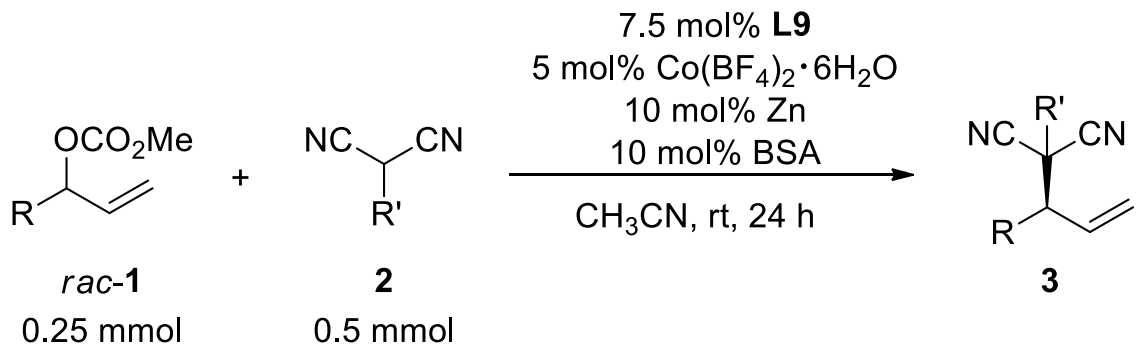

In an $\mathrm{N}_{2}$ filled glove box, $\mathbf{L 9}\left(8.0 \mathrm{mg}, 18.8 \times 10^{-3} \mathrm{mmol}, 7.5 \mathrm{~mol} \%\right)$ and $\mathrm{Co}\left(\mathrm{BF}_{4}\right)_{2} \cdot 6 \mathrm{H}_{2} \mathrm{O}\left(4.3 \mathrm{mg}, 12.6 \times 10^{-3} \mathrm{mmol}\right.$, $5 \mathrm{~mol} \%$ ) in $0.2 \mathrm{~mL}$ acetonitrile was transferred into a $10 \mathrm{~mL}$ reaction tube equipped with a Teflon stopcock and a stir bar. Zn dust $\left(1.7 \mathrm{mg}, 26.0 \times 10^{-3} \mathrm{mmol}, 10 \mathrm{~mol} \%\right)$ was added followed by addition of $0.5 \mathrm{~mL}$ acetonitrile to complete transfer of zinc. The mixture was allowed to stir for 20 minutes. Afterward, allylic carbonate $(0.25$ mmol), malonitrile derivatives ( $0.5 \mathrm{mmol}, 2 \mathrm{eq})$, bis(trimethylsilyl)acetamide (BSA) $\left(6 \mu \mathrm{L}, 25 \times 10^{-3} \mathrm{mmol}, 10\right.$ mol\%), and $0.3 \mathrm{~mL}$ of acetonitrile were added sequentially to the reaction mixture. The reaction tube was sealed and brought out from the glove box. The reaction mixture was then stirred for 24 hours at room temperature. Then, solvent was removed and column chromatography was run on a column of silica gels (200-300 mesh) using PE/EA as an eluent to get the desired product.

\section{Large scale synthesis of 3aa using 2.5 mol\% catalyst:}

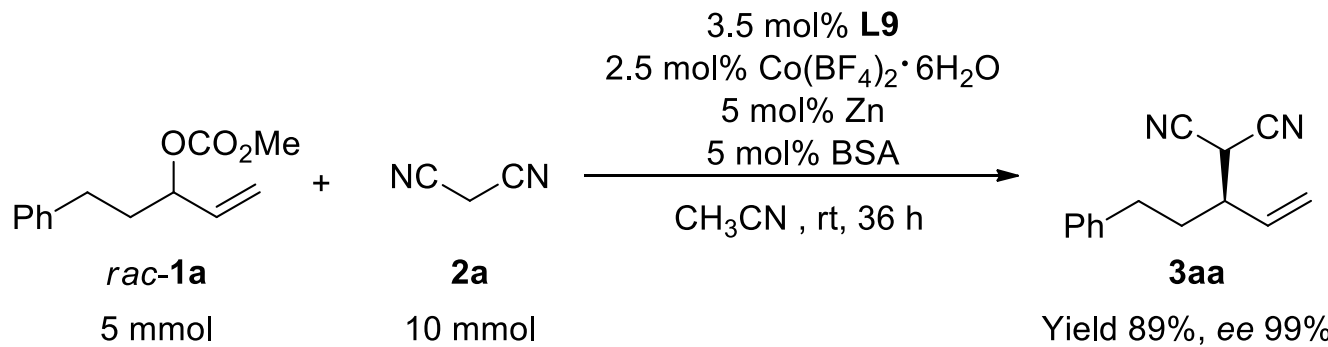

In an $\mathrm{N}_{2}$ filled glove box, a reaction tube equipped with a Teflon stopcock and a stir bar was charged with 3.5 mol\% of $\mathbf{L 9}(75 \mathrm{mg}, 0.18 \mathrm{mmol})$, and $2.5 \mathrm{~mol} \%$ of $\mathrm{Co}\left(\mathrm{BF}_{4}\right)_{2} \cdot 6 \mathrm{H}_{2} \mathrm{O}(43 \mathrm{mg}, 0.126 \mathrm{mmol})$ in $1 \mathrm{~mL}$ of acetonitrile. Zn dust (16.4 mg, $0.25 \mathrm{mmol}, 5 \mathrm{~mol} \%$ ) was added followed by addition of $1 \mathrm{~mL}$ acetonitrile to complete transfer of zinc. The mixture was stirred for 20 minutes. Then, $r a c-1 a(1.102 \mathrm{~g}, 5 \mathrm{mmol})$ and $\mathbf{2 a}(0.660 \mathrm{~g}, 10 \mathrm{mmol})$, bis(trimethylsilyl)acetamide (BSA) $(60 \mu \mathrm{L}, 5 \mathrm{~mol} \%)$ were added to the reaction mixture sequentially. Then, the reaction was monitored by TLC and stopped after 36 hours. The reaction tube was removed from the glove box and solvent was removed under reduced pressure. The residue was purified by flash column chromatography using PE/EA as an eluent. Yield: $0.930 \mathrm{~g}, 89 \%$; ee: $99 \%$. 


\section{Spectral data of allylic alkylation of malonitrile products:}

\section{(R)-2-(5-phenylpent-1-en-3-yl)malononitrile (3aa): ${ }^{5,6}$}

Clear oil; Yield: $47 \mathrm{mg}, 89 \%$.<smiles>C=CC(CCc1ccccc1)C(C#N)C#N</smiles>

TLC Rf $=0.5$ (PE:EA, 9:1).

${ }^{1} \mathbf{H}$ NMR $\left(400 \mathrm{MHz}, \mathrm{CDCl}_{3}\right) \delta 7.33(\mathrm{t}, J=7.3 \mathrm{~Hz}, 2 \mathrm{H}), 7.25(\mathrm{t}, J=7.3 \mathrm{~Hz}, 1 \mathrm{H}), 7.20-$ $7.18(\mathrm{~m}, 2 \mathrm{H}), 5.79-5.70(\mathrm{~m}, 1 \mathrm{H}), 5.45(\mathrm{dd}, J=32.7,13.5 \mathrm{~Hz}, 2 \mathrm{H}), 3.68(\mathrm{~d}, J=5.4 \mathrm{~Hz}$, $1 \mathrm{H}), 2.79$ (ddd, $J=14.0,8.8,5.3 \mathrm{~Hz}, 1 \mathrm{H}), 2.68-2.55(\mathrm{~m}, 2 \mathrm{H}), 2.08-1.90(\mathrm{~m}, 2 \mathrm{H})$.

${ }^{13}$ C NMR (101 MHz, $\left.\mathrm{CDCl}_{3}\right) \delta 140.1,133.8,128.9,128.5,126.7,122.3,111.9,111.7,44.4,33.3,32.8,28.8$.

HPLC (Shimadzu LC-2030) (Daicel Chiralpak OD-H Column, ${ }^{i} \mathrm{PrOH}: n-\mathrm{Hexane}=10.0: 90.0,1.0 \mathrm{~mL} / \mathrm{min}$ ), R $t$ $=12.95 \mathrm{~min}$ (major) and $16.04 \mathrm{~min}$ (minor), $99 \%$ ee.

$[\boldsymbol{\alpha}]_{D}^{25}=-21.6\left(c 0.05, \mathrm{CHCl}_{3}\right)$.

HRMS (ESI): $m / z[\mathrm{M}+\mathrm{K}]^{+}$Calcd for $\mathrm{C}_{14} \mathrm{H}_{14} \mathrm{~N}_{2} \mathrm{~K}=249.0794$; found 249.0710.

\section{Assignment of absolute configuration:}

Literature data ${ }^{4}$ for $(R)$-enantiomer: $[\boldsymbol{\alpha}]_{D}^{24}-8.05\left(c=0.77, \mathrm{CHCl}_{3}\right), 82 \%$ ee

Literature data ${ }^{5}$ for $(S)$-enantiomer: $[\boldsymbol{\alpha}]_{D}^{25}+21.7\left(c=1.28, \mathrm{CHCl}_{3}\right), 88 \%$ ee

By comparing the literature reported data, the absolute configuration of the product 3aa was assigned as $(\boldsymbol{R})$ and all other compounds were assigned by analogy.

\section{(R)-2-benzyl-2-(5-phenylpent-1-en-3-yl)malononitrile (3ab): 5}

White solid; Yield: $65 \mathrm{mg}, 87 \%$; M.P. $79-81^{\circ} \mathrm{C}$.

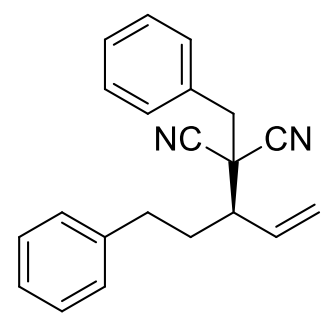

TLC R $\boldsymbol{f}=0.6$ (PE:EA, 9:1).

${ }^{1} \mathbf{H}$ NMR $\left(400 \mathrm{MHz}, \mathrm{CDCl}_{3}\right) \delta 7.42-7.34(\mathrm{~m}, 7 \mathrm{H}), 7.29-7.22(\mathrm{~m}, 3 \mathrm{H}), 5.82(\mathrm{dt}, J=$ $16.9,9.9 \mathrm{~Hz}, 1 \mathrm{H}), 5.61(\mathrm{dd}, J=10.2,1.1 \mathrm{~Hz}, 1 \mathrm{H}), 5.41(\mathrm{~d}, J=17.0 \mathrm{~Hz}, 1 \mathrm{H}), 3.22(\mathrm{~d}, J$ $=13.7 \mathrm{~Hz}, 1 \mathrm{H}), 3.04(\mathrm{~d}, J=13.8 \mathrm{~Hz}, 1 \mathrm{H}), 2.88(\mathrm{ddd}, J=13.8,9.1,4.6 \mathrm{~Hz}, 1 \mathrm{H}), 2.62-$ $2.52(\mathrm{~m}, 2 \mathrm{H}), 2.38-2.30(\mathrm{~m}, 1 \mathrm{H}), 2.07-1.98(\mathrm{~m}, 1 \mathrm{H})$.

${ }^{13}$ C NMR (126 MHz, $\left.\mathrm{CDCl}_{3}\right) \delta 140.3,133.4,132.5,130.3,129.0,128.8,128.6,126.5,123.5,115.0,114.3,50.2$, 44.4, 41.7, 32.9, 32.4 .

HPLC (Shimadzu LC-16) (Daicel Chiralpak IG Column, ${ }^{i} \mathrm{PrOH}: n-\mathrm{Hexane}=1.0: 99.0,1.0 \mathrm{~mL} / \mathrm{min}$ ), R $t=17.25$ $\min$ (minor) and $20.31 \mathrm{~min}$ (major), > 99\% ee.

$[\alpha]_{D}^{25}=-26.4\left(c 0.05, \mathrm{CHCl}_{3}\right)$. Literature data ${ }^{4}$ for $(R)$-enantiomer: $[\alpha]_{D}^{26}-2.4\left(c=0.5, \mathrm{CHCl}_{3}\right)$.

HRMS (ESI): $m / z[\mathrm{M}+\mathrm{Na}]^{+}$Calcd for $\mathrm{C}_{21} \mathrm{H}_{20} \mathrm{~N}_{2} \mathrm{Na}$ 323.1524; found 323.1525.

\section{(R)-2-(4-cyanobenzyl)-2-(5-phenylpent-1-en-3-yl)malononitrile (3ac):}

White solid; Yield: $71 \mathrm{mg}, 87 \%$; M.P. $131-132{ }^{\circ} \mathrm{C}$. 


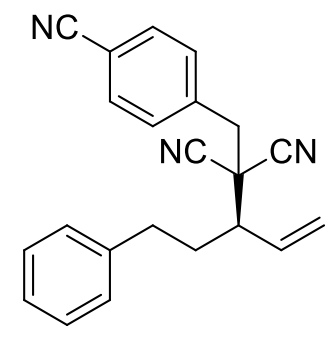

TLC R $\boldsymbol{f}=0.3$ (PE:EA, 9:1).

${ }^{1} \mathbf{H}$ NMR $\left(400 \mathrm{MHz}, \mathrm{CDCl}_{3}\right) \delta 7.67(\mathrm{~d}, J=8.3 \mathrm{~Hz}, 2 \mathrm{H}), 7.46(\mathrm{~d}, J=8.3 \mathrm{~Hz}, 2 \mathrm{H}), 7.32$

$(\mathrm{t}, J=7.3 \mathrm{~Hz}, 2 \mathrm{H}), 7.25-7.22(\mathrm{~m}, 1 \mathrm{H}), 7.18(\mathrm{~d}, J=7.1 \mathrm{~Hz}, 2 \mathrm{H}), 5.77(\mathrm{dt}, J=16.8,9.9$ $\mathrm{Hz}, 1 \mathrm{H}), 5.61(\mathrm{dd}, J=10.2,0.9 \mathrm{~Hz}, 1 \mathrm{H}), 5.40(\mathrm{~d}, J=16.7 \mathrm{~Hz}, 1 \mathrm{H}), 3.23(\mathrm{~d}, J=13.7 \mathrm{~Hz}$, $1 \mathrm{H}), 3.03(\mathrm{~d}, J=13.7 \mathrm{~Hz}, 1 \mathrm{H}), 2.86(\mathrm{ddd}, J=13.6,8.7,4.6 \mathrm{~Hz}, 1 \mathrm{H}), 2.54(\mathrm{dt}, J=22.3$, $10.2 \mathrm{~Hz}, 2 \mathrm{H}), 2.33-2.25(\mathrm{~m}, 1 \mathrm{H}), 2.05-1.95(\mathrm{~m}, 1 \mathrm{H})$.

${ }^{13} \mathbf{C ~ N M R}\left(126 \mathrm{MHz}, \mathrm{CDCl}_{3}\right) \delta 140.1,137.7,133.0,132.8,131.2,128.9,128.6,126.7,124.1,118.4,114.5,113.8$, 113.1, 50.4, 44.0, 41.7, 32.8, 32.5 .

HPLC (Shimadzu LC-2030) (Daicel Chiralpak OD-H Column, ${ }^{i} \mathrm{PrOH}: n-\mathrm{Hexane}=2.0: 98.0,1.0 \mathrm{~mL} / \mathrm{min}$ ), R $t=$ $49.73 \mathrm{~min}$ (minor) and 53.14 min (major), > 99\% ee.

$[\alpha]_{D}^{25}=-2.6\left(c 0.05, \mathrm{CHCl}_{3}\right)$.

HRMS (ESI): $m / z[\mathrm{M}+\mathrm{Na}]^{+}$Calcd for $\mathrm{C}_{22} \mathrm{H}_{19} \mathrm{~N}_{3} \mathrm{Na} 348.1477$; found 348.1474.

\section{(R)-2-(5-phenylpent-1-en-3-yl)-2-(4-(trifluoromethyl)benzyl)malononitrile (3ad):}

White solid; Yield: $80 \mathrm{mg}, 87 \%$; M.P. $120-122{ }^{\circ} \mathrm{C}$.

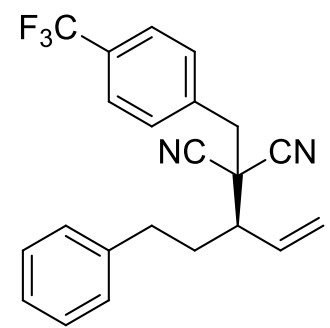

TLC R $\boldsymbol{f}=0.7$ (PE:EA, 9:1).

${ }^{1} \mathbf{H}$ NMR $\left(400 \mathrm{MHz}, \mathrm{CDCl}_{3}\right) \delta 7.64(\mathrm{~d}, J=8.1 \mathrm{~Hz}, 2 \mathrm{H}), 7.47(\mathrm{~d}, J=8.1 \mathrm{~Hz}, 2 \mathrm{H}), 7.32$ $(\mathrm{t}, J=7.3 \mathrm{~Hz}, 2 \mathrm{H}), 7.25-7.18(\mathrm{~m}, 3 \mathrm{H}), 5.78(\mathrm{dt}, J=16.9,9.9 \mathrm{~Hz}, 1 \mathrm{H}), 5.61(\mathrm{dd}, J=$ $10.2,1.2 \mathrm{~Hz}, 1 \mathrm{H}), 5.39(\mathrm{dd}, J=16.9,0.6 \mathrm{~Hz}, 1 \mathrm{H}), 3.23(\mathrm{~d}, J=13.7 \mathrm{~Hz}, 1 \mathrm{H}), 3.04(\mathrm{~d}, J$ $=13.7 \mathrm{~Hz}, 1 \mathrm{H}), 2.86(\mathrm{ddd}, J=13.7,8.8,4.6 \mathrm{~Hz}, 1 \mathrm{H}), 2.60-2.48(\mathrm{~m}, 2 \mathrm{H}), 2.34-2.26$ (m, 1H), $2.05-1.95(\mathrm{~m}, 1 \mathrm{H})$.

${ }^{13} \mathrm{C} \mathrm{NMR}\left(126 \mathrm{MHz}, \mathrm{CDCl}_{3}\right) \delta 140.2,136.5,133.2,131.6$ - 130.8 (m), 130.8, 128.9, 128.6, 126.7, 126.1 (q, $J$ $=3.7 \mathrm{~Hz}), 127.3-120.8(\mathrm{~m}), 124.0,114.7,114.0,50.4,44.1,41.5,32.8,32.5$.

HPLC (Shimadzu LC-2030) (Daicel Chiralpak OD-H Column, ${ }^{i} \mathrm{PrOH}: n-\mathrm{Hexane}=0.1: 99.9,0.5 \mathrm{~mL} / \mathrm{min}$ ), R $t=$ $57.50 \mathrm{~min}$ (minor) and $61.26 \mathrm{~min}$ (major), > 99\% ee.

$[\alpha]_{D}^{25}=-1.0\left(c 0.05, \mathrm{CHCl}_{3}\right)$.

HRMS (ESI): $m / z$ [M + Na $]^{+}$Calcd for $\mathrm{C}_{22} \mathrm{H}_{19} \mathrm{~F}_{3} \mathrm{~N}_{2} \mathrm{Na}$ 391.1398; found 391.1387.

\section{(R)-2-(naphthalen-2-ylmethyl)-2-(5-phenylpent-1-en-3-yl)malononitrile (3ae):}

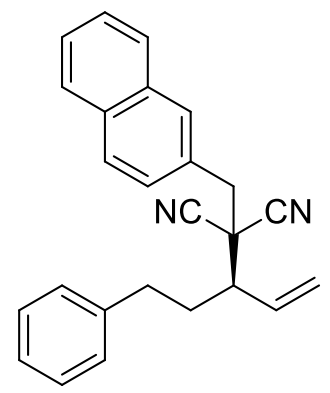

White solid; Yield: $80 \mathrm{mg}, 91 \%$; M.P. $103-105^{\circ} \mathrm{C}$.

TLC R $\boldsymbol{f}=0.3$ (PE:EA, 9:1).

${ }^{1} \mathbf{H}$ NMR $\left(500 \mathrm{MHz}, \mathrm{CDCl}_{3}\right) \delta 7.84(\mathrm{dd}, J=9.2,3.8 \mathrm{~Hz}, 4 \mathrm{H}), 7.52-7.48(\mathrm{~m}, 2 \mathrm{H}), 7.45$ $(\mathrm{dd}, J=8.5,1.8 \mathrm{~Hz}, 1 \mathrm{H}), 7.31(\mathrm{t}, J=7.4 \mathrm{~Hz}, 2 \mathrm{H}), 7.23(\mathrm{~d}, J=8.2 \mathrm{~Hz}, 1 \mathrm{H}), 7.21-7.18$ $(\mathrm{m}, 2 \mathrm{H}), 5.81(\mathrm{dt}, J=16.9,10.0 \mathrm{~Hz}, 1 \mathrm{H}), 5.60(\mathrm{dd}, J=10.2,1.0 \mathrm{~Hz}, 1 \mathrm{H}), 5.40(\mathrm{~d}, J=$ 
$16.9 \mathrm{~Hz}, 1 \mathrm{H}), 3.35(\mathrm{~d}, J=13.8 \mathrm{~Hz}, 1 \mathrm{H}), 3.17(\mathrm{~d}, J=13.8 \mathrm{~Hz}, 1 \mathrm{H}), 2.84(\mathrm{ddd}, J=13.8,9.2,4.6 \mathrm{~Hz}, 1 \mathrm{H}), 2.58-$ $2.52(\mathrm{~m}, 2 \mathrm{H}), 2.35-2.30(\mathrm{~m}, 1 \mathrm{H}), 2.04-1.97(\mathrm{~m}, 1 \mathrm{H})$.

${ }^{13} \mathrm{C} \mathrm{NMR}\left(126 \mathrm{MHz}, \mathrm{CDCl}_{3}\right) \delta 140.4,133.5,133.4,133.3,130.0,129.8,128.9$ (2C), 128.6, 128.2, 127.9, 127.7, $126.8,126.7,126.6,123.7,115.1,114.4,50.4,44.5,42.0,32.9,32.5$.

HPLC (Shimadzu LC-2030) (Daicel Chiralpak OD-H Column, ${ }^{i}$ PrOH: $n$-Hexane = 8.0:92.0, $1.0 \mathrm{~mL} / \mathrm{min}$ ), R $t=$ $14.82 \mathrm{~min}$ (minor) and $24.01 \mathrm{~min}$ (major), $99 \%$ ee.

$[\alpha]_{D}^{25}=-2.6\left(c 0.05, \mathrm{CHCl}_{3}\right)$.

HRMS (ESI): $m / z[\mathrm{M}+\mathrm{Na}]^{+}$Calcd for $\mathrm{C}_{25} \mathrm{H}_{22} \mathrm{~N}_{2} \mathrm{Na} 373.1681$; found 373.1682.

\section{(R)-2-((1-methyl-1H-pyrrol-2-yl)methyl)-2-(5-phenylpent-1-en-3-yl)malononitrile (3af):}

Clear oil; Yield: $65 \mathrm{mg}, 86 \%$.

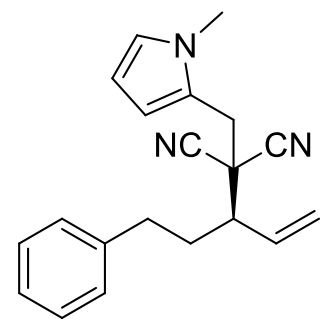

TLC R $\boldsymbol{f}=0.4$ (PE:EA, 9:1).

${ }^{1} \mathbf{H}$ NMR $\left(400 \mathrm{MHz}, \mathrm{CDCl}_{3}\right) \delta 7.31(\mathrm{t}, J=7.3 \mathrm{~Hz}, 2 \mathrm{H}), 7.25-7.18(\mathrm{~m}, 3 \mathrm{H}), 6.62(\mathrm{dd}, J$ $=2.5,1.9 \mathrm{~Hz}, 1 \mathrm{H}), 6.24(\mathrm{dd}, J=3.6,1.7 \mathrm{~Hz}, 1 \mathrm{H}), 6.12-6.10(\mathrm{~m}, 1 \mathrm{H}), 5.76(\mathrm{dt}, J=16.9$, $9.9 \mathrm{~Hz}, 1 \mathrm{H}), 5.58(\mathrm{dd}, J=10.2,1.1 \mathrm{~Hz}, 1 \mathrm{H}), 5.39(\mathrm{~d}, J=17.0 \mathrm{~Hz}, 1 \mathrm{H}), 3.63(\mathrm{~s}, 3 \mathrm{H}), 3.26$ (d, $J=15.2 \mathrm{~Hz}, 1 \mathrm{H}), 3.09$ (d, $J=15.2 \mathrm{~Hz}, 1 \mathrm{H}), 2.83(\mathrm{ddd}, J=13.9,9.3,4.7 \mathrm{~Hz}, 1 \mathrm{H})$, $2.59-2.51(\mathrm{~m}, 2 \mathrm{H}), 2.32-2.23(\mathrm{~m}, 1 \mathrm{H}), 2.02-1.92(\mathrm{~m}, 1 \mathrm{H})$.

${ }^{13} \mathrm{C} \mathrm{NMR}\left(126 \mathrm{MHz}, \mathrm{CDCl}_{3}\right) \delta 140.4,133.4,128.9,128.6,126.6,124.1,123.7,123.0,115.3,114.6,111.1,107.9$, $49.8,43.8,34.3,32.9,32.7,32.5$.

HPLC (Shimadzu LC-2030) (Daicel Chiralpak OD-H Column, ${ }^{i}$ PrOH: $n$-Hexane = 5.0:95.0, $1.0 \mathrm{~mL} / \mathrm{min}$ ), R $t=$ $18.29 \mathrm{~min}$ (major) and $21.90 \mathrm{~min}$ (minor), $99 \%$ ee.

$[\alpha]_{D}^{25}=-9.6\left(c 0.05, \mathrm{CHCl}_{3}\right)$.

HRMS (ESI): $m / z[\mathrm{M}+\mathrm{H}]^{+}$Calcd for $\mathrm{C}_{20} \mathrm{H}_{22} \mathrm{~N}_{3}$ 304.1808; found 304.1815.

\section{(R)-2-(furan-2-ylmethyl)-2-(5-phenylpent-1-en-3-yl)malononitrile (3ag):}

Clear oil; Yield: $60 \mathrm{mg}, 83 \%$.

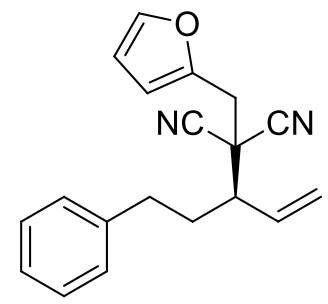

TLC R $\boldsymbol{f}=0.5$ (PE:EA, 9:1).

${ }^{1} \mathbf{H}$ NMR $\left(400 \mathrm{MHz}, \mathrm{CDCl}_{3}\right) \delta 7.41(\mathrm{dd}, J=1.8,0.7 \mathrm{~Hz}, 1 \mathrm{H}), 7.32(\mathrm{t}, J=7.3 \mathrm{~Hz}, 2 \mathrm{H})$, $7.25-7.18(\mathrm{~m}, 3 \mathrm{H}), 6.40-6.37(\mathrm{~m}, 2 \mathrm{H}), 5.74(\mathrm{dt}, J=16.9,9.9 \mathrm{~Hz}, 1 \mathrm{H}), 5.58(\mathrm{dd}, J=$ $10.2,1.2 \mathrm{~Hz}, 1 \mathrm{H}), 5.40(\mathrm{dd}, J=16.9,0.7 \mathrm{~Hz}, 1 \mathrm{H}), 3.28(\mathrm{dd}, J=46.9,15.1 \mathrm{~Hz}, 2 \mathrm{H}), 2.83$ (ddd, $J=13.9,9.3,4.6 \mathrm{~Hz}, 1 \mathrm{H}), 2.58-2.45(\mathrm{~m}, 2 \mathrm{H}), 2.32-2.23(\mathrm{~m}, 1 \mathrm{H}), 2.01-1.92$ $(\mathrm{m}, 1 \mathrm{H})$.

${ }^{13}$ C NMR (126 MHz, $\left.\mathrm{CDCl}_{3}\right) \delta 146.5,143.6,140.4,133.1,128.8,128.6,126.5,123.9,114.7,114.2,111.0,110.8$, 49.3, 42.4, $34.732 .9,32.3$. 
HPLC (Shimadzu LC-16) (Daicel Chiralpak IC Column, ${ }^{i} \mathrm{PrOH}: n-H e x a n e=0.5: 99.5,1.0 \mathrm{~mL} / \mathrm{min}$ ), R $t=20.69$ $\min$ (minor) and $22.48 \mathrm{~min}$ (major), > 99\% ee.

$[\alpha]_{D}^{25}=-6.3\left(c 0.05, \mathrm{CHCl}_{3}\right)$.

HRMS (ESI): $m / z[\mathrm{M}+\mathrm{Na}]^{+}$Calcd for $\mathrm{C}_{19} \mathrm{H}_{18} \mathrm{~N}_{2} \mathrm{ONa}$ 313.1317; found 313.1321.

\section{(R)-2-(cyclohexylmethyl)-2-(5-phenylpent-1-en-3-yl)malononitrile (3ah):}

Clear oil; Yield: $67.5 \mathrm{mg}, 88 \%$.

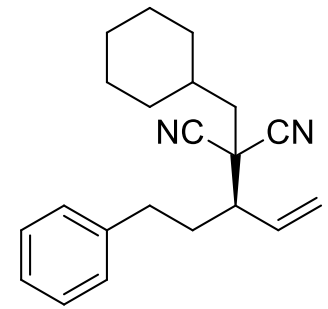

TLC R $\boldsymbol{f}=0.7$ (PE:EA, 9:1).

${ }^{1} \mathbf{H}$ NMR $\left(400 \mathrm{MHz}, \mathrm{CDCl}_{3}\right) \delta 7.31(\mathrm{t}, J=7.3 \mathrm{~Hz}, 2 \mathrm{H}), 7.20(\mathrm{dd}, J=20.4,7.2 \mathrm{~Hz}, 3 \mathrm{H})$, $5.66(\mathrm{dt}, J=16.9,9.9 \mathrm{~Hz}, 1 \mathrm{H}), 5.49(\mathrm{dd}, J=10.2,1.2 \mathrm{~Hz}, 1 \mathrm{H}), 5.30(\mathrm{dd}, J=16.9,0.8$ $\mathrm{Hz}, 1 \mathrm{H}), 2.82$ (ddd, $J=13.8,9.0,4.6 \mathrm{~Hz}, 1 \mathrm{H}), 2.56-2.48(\mathrm{~m}, 1 \mathrm{H}), 2.40-2.34(\mathrm{~m}, 1 \mathrm{H})$, $2.27-2.19(\mathrm{~m}, 1 \mathrm{H}), 1.97-1.85(\mathrm{~m}, 3 \mathrm{H}), 1.81-1.63(\mathrm{~m}, 6 \mathrm{H}), 1.31-1.24(\mathrm{~m}, 2 \mathrm{H}), 1.18$ $-1.12(\mathrm{~m}, 1 \mathrm{H}), 1.09-0.98(\mathrm{~m}, 2 \mathrm{H})$.

${ }^{13} \mathrm{C} \mathrm{NMR}\left(126 \mathrm{MHz}, \mathrm{CDCl}_{3}\right) \delta 140.5,133.5,128.8,128.6,126.6,123.2,115.8,115.3,51.4,43.0,40.4,35.9$, 33.7, 33.5, 32.9, 32.1, 26.1, 26.1, 26.0.

HPLC (Shimadzu LC-2030) (Daicel Chiralpak OD-H Column, ${ }^{i} \operatorname{PrOH}: n-H e x a n e=1.0: 99.0,1.0 \mathrm{~mL} / \mathrm{min}$ ), R $t=$ $6.88 \mathrm{~min}$ (major) and $7.66 \mathrm{~min}$ (minor), $96 \%$ ee.

$[\boldsymbol{\alpha}]_{\boldsymbol{D}}^{25}=-14.1\left(c 0.05, \mathrm{CHCl}_{3}\right)$.

HRMS (ESI): $m / z[\mathrm{M}+\mathrm{Na}]^{+}$Calcd for $\mathrm{C}_{21} \mathrm{H}_{26} \mathrm{~N}_{2} \mathrm{Na}$ 329.1994; found 329.1992.

\section{(R)-2-allyl-2-(5-phenylpent-1-en-3-yl)malononitrile (3ai): ${ }^{5}$}

Clear oil; Yield: $53 \mathrm{mg}, 85 \%$.

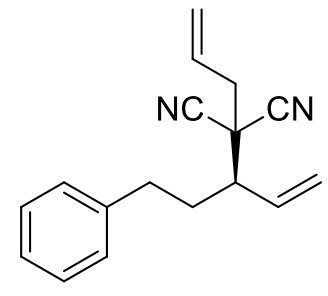

TLC R $\boldsymbol{f}=0.6$ (PE:EA, 9:1).

${ }^{1} \mathbf{H}$ NMR $\left(400 \mathrm{MHz}, \mathrm{CDCl}_{3}\right) \delta 7.33(\mathrm{t}, J=7.3 \mathrm{~Hz}, 2 \mathrm{H}), 7.26-7.19(\mathrm{~m}, 3 \mathrm{H}), 5.92-5.81$ $(\mathrm{m}, 1 \mathrm{H}), 5.70(\mathrm{dt}, J=16.8,9.9 \mathrm{~Hz}, 1 \mathrm{H}), 5.54(\mathrm{dd}, J=10.2,1.2 \mathrm{~Hz}, 1 \mathrm{H}), 5.41-5.32(\mathrm{~m}$, $3 \mathrm{H}), 2.87-2.80(\mathrm{~m}, 1 \mathrm{H}), 2.69(\mathrm{dd}, J=14.0,7.1 \mathrm{~Hz}, 1 \mathrm{H}), 2.59-2.43(\mathrm{~m}, 3 \mathrm{H}), 2.28-$ $2.20(\mathrm{~m}, 1 \mathrm{H}), 2.00-1.91(\mathrm{~m}, 1 \mathrm{H})$.

${ }^{13} \mathrm{C}$ NMR $\left(101 \mathrm{MHz}, \mathrm{CDCl}_{3}\right) \delta 140.3,133.1,128.8,128.6,126.5,123.4,123.2,114.9,114.3,49.2,42.2,40.1$, $32.8,32.3$.

HPLC (Shimadzu LC-2030) (Daicel Chiralpak OD-H Column, ${ }^{i} \mathrm{PrOH}: n-\mathrm{Hex} a n e=5.0: 95.0,1.0 \mathrm{~mL} / \mathrm{min}$ ), R $t=$ $11.66 \mathrm{~min}$ (minor) and $12.65 \mathrm{~min}$ (major), > 99\% ee.

$[\alpha]_{D}^{25}=-6.3\left(c 0.05, \mathrm{CHCl}_{3}\right)$. Literature data ${ }^{4}$ for $(R)$-enantiomer: $[\boldsymbol{\alpha}]_{\boldsymbol{D}}^{26}=-2.78\left(c=0.5, \mathrm{CHCl}_{3}\right), 71 \%$ ee HRMS (ESI): $m / z[\mathrm{M}+\mathrm{Na}]^{+}$Calcd for $\mathrm{C}_{17} \mathrm{H}_{18} \mathrm{~N}_{2} \mathrm{Na}$ 273.1368; found 273.1370. 


\section{(R)-2-(5-phenylpent-1-en-3-yl)-2-(prop-2-yn-1-yl)malononitrile (3aj):}

Clear oil; Yield: $47 \mathrm{mg}, 76 \%$.

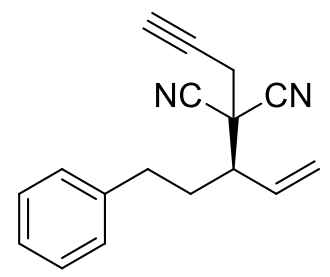

TLC R $\boldsymbol{f}=0.6$ (PE:EA, 9:1).

${ }^{1} \mathbf{H}$ NMR $\left(400 \mathrm{MHz}, \mathrm{CDCl}_{3}\right) \delta 7.32(\mathrm{t}, J=7.3 \mathrm{~Hz}, 2 \mathrm{H}), 7.22(\mathrm{dd}, J=18.6,7.2 \mathrm{~Hz}, 3 \mathrm{H})$, $5.71-5.62(\mathrm{~m}, 1 \mathrm{H}), 5.55(\mathrm{dd}, J=10.1,1.5 \mathrm{~Hz}, 1 \mathrm{H}), 5.43(\mathrm{dd}, J=16.7,1.2 \mathrm{~Hz}, 1 \mathrm{H})$, $2.97-2.77(\mathrm{~m}, 3 \mathrm{H}), 2.68(\mathrm{ddd}, J=12.0,9.6,2.7 \mathrm{~Hz}, 1 \mathrm{H}), 2.60-2.52(\mathrm{~m}, 1 \mathrm{H}), 2.34(\mathrm{t}$, $J=2.6 \mathrm{~Hz}, 1 \mathrm{H}), 2.29-2.20(\mathrm{~m}, 1 \mathrm{H}), 2.01-1.91(\mathrm{~m}, 1 \mathrm{H})$.

${ }^{13} \mathrm{C}$ NMR $\left(126 \mathrm{MHz}, \mathrm{CDCl}_{3}\right) \delta 140.2,132.4,128.8,128.6,128.6,126.6,124.1,114.3,113.7,75.3,74.7,48.6$, 41.3, 32.9, 32.3, 27.3.

HPLC (Shimadzu LC-2030) (Daicel Chiralpak OD-H Column, ${ }^{i} \mathrm{PrOH}: n-H e x a n e=5.0: 95.0,1.0 \mathrm{~mL} / \mathrm{min}$ ), R $t=$ $8.32 \mathrm{~min}$ (minor) and $10.60 \mathrm{~min}$ (major), > 99\% ee.

$[\boldsymbol{\alpha}]_{D}^{25}=-11.1\left(c 0.05, \mathrm{CHCl}_{3}\right)$.

HRMS (ESI): $m / z$ [M + Na $]^{+}$Calcd for $\mathrm{C}_{17} \mathrm{H}_{16} \mathrm{~N}_{2} \mathrm{Na}$ 271.1211; found 271.1210.

\section{(R)-methyl 2-cyano-3-phenethylpent-4-enoate (3ak):}

Clear oil; Yield: 34 mg, 56\%.

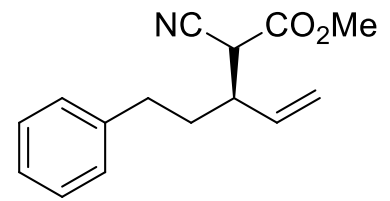

TLC R $\boldsymbol{f}=0.3$ (PE:EA, 9:1).

${ }^{1} \mathbf{H}$ NMR $\left(500 \mathrm{MHz}, \mathrm{CDCl}_{3}\right) \delta 7.30(\mathrm{t}, J=7.4 \mathrm{~Hz}, 2 \mathrm{H}), 7.23-7.16(\mathrm{~m}, 3 \mathrm{H}), 5.79-$ $5.69(\mathrm{~m}, 1 \mathrm{H}), 5.31-5.20(\mathrm{~m}, 2 \mathrm{H}), 3.77(\mathrm{~s}, 3 \mathrm{H}),\{3.59(\mathrm{~d}, J=4.8 \mathrm{~Hz}, 0.6 \mathrm{H}), 3.52(\mathrm{~d}$, $J=6.0 \mathrm{~Hz}, 0.4 \mathrm{H}) ; d / r=1.0: 1.5.\}, 2.81-2.69(\mathrm{~m}, 2 \mathrm{H}), 2.55(\mathrm{ddd}, J=25.8,15.2,8.4$

$\mathrm{Hz}, 1 \mathrm{H}), 1.97-1.80(\mathrm{~m}, 2 \mathrm{H})$.

${ }^{13} \mathrm{C}$ NMR $\left(126 \mathrm{MHz}, \mathrm{CDCl}_{3}\right) \delta 165.9,165.8,141.0,140.9,136.3,135.4,128.7,128.7,128.5,126.4,126.3,120.2$, 119.7, 115.4, 115.1, 53.5, 53.5, 44.2, 44.1, 43.5, 43.5, 34.4, 33.2, 33.1, 33.0.

HPLC (Shimadzu LC-2030) (Daicel Chiralpak IC Column, ${ }^{i} \mathrm{PrOH}: n$-Hexane $=0.1: 99.1,1.0 \mathrm{~mL} / \mathrm{min}$ ), $d / r=$ 1.0:1.5. $\mathrm{R} t=40.78 \mathrm{~min}$ (minor) and $46.95 \mathrm{~min}$ (major), $>99 \%$ ee for the major diastereomer.

$[\alpha]_{D}^{25}=-5.3\left(c 0.05, \mathrm{CHCl}_{3}\right)$.

HRMS (ESI): $m / z[\mathrm{M}+\mathrm{Na}]^{+} \mathrm{Calcd}$ for $\mathrm{C}_{15} \mathrm{H}_{17} \mathrm{NO}_{2} \mathrm{Na} 266.1157$; found 266.1158 .

\section{(R)-(2-vinylpentane-1,1-diyldisulfonyl)dibenzene (3al):}

White solid; Yield: $58 \mathrm{mg}, 61 \%$; M.P. $91-93{ }^{\circ} \mathrm{C}$.

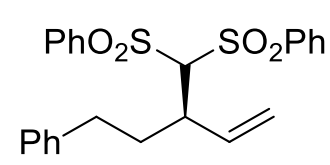

TLC R $\boldsymbol{f}=0.2$ (PE:EA, 9:1).

${ }^{1} \mathbf{H}$ NMR $\left(500 \mathrm{MHz}, \mathrm{CDCl}_{3}\right) \delta 7.93(\mathrm{t}, J=8.1 \mathrm{~Hz}, 4 \mathrm{H}), 7.70-7.64(\mathrm{~m}, 2 \mathrm{H}), 7.54(\mathrm{dd}$, $J=17.3,8.0 \mathrm{~Hz}, 4 \mathrm{H}), 6.12(\mathrm{dt}, J=17.2,9.5 \mathrm{~Hz}, 1 \mathrm{H}), 5.07(\mathrm{~d}, J=10.1 \mathrm{~Hz}, 1 \mathrm{H}), 4.92(\mathrm{~d}$, $J=17.1 \mathrm{~Hz}, 1 \mathrm{H}), 4.60(\mathrm{~s}, 1 \mathrm{H}), 3.06-3.01(\mathrm{~m}, 1 \mathrm{H}), 1.90(\mathrm{ddd}, J=23.8,10.3,4.8 \mathrm{~Hz}, 1 \mathrm{H}), 1.63-1.56(\mathrm{~m}, 1 \mathrm{H})$, $1.34-1.28(\mathrm{~m}, 1 \mathrm{H}), 1.11-1.04(\mathrm{~m}, 1 \mathrm{H}), 0.80(\mathrm{t}, J=7.3 \mathrm{~Hz}, 3 \mathrm{H})$. 
${ }^{13} \mathrm{C}$ NMR $\left(126 \mathrm{MHz}, \mathrm{CDCl}_{3}\right) \delta 140.0,139.0,137.7,134.7,134.4,129.8,129.5,129.3,129.2,118.4,88.0,44.5$, 32.7, 21.5, 13.7 .

HPLC (Shimadzu LC-2030) (Daicel Chiralpak IG Column, ${ }^{i} \mathrm{PrOH}: n-\mathrm{Hexane}=3.0: 97.0,0.5 \mathrm{~mL} / \mathrm{min}$ ), R $t=$ $86.38 \mathrm{~min}$ (major) and $90.37 \mathrm{~min}$ (minor), > 99\% ee.

$[\alpha]_{D}^{25}=+7.6\left(c 0.05, \mathrm{CHCl}_{3}\right)$.

HRMS (ESI): $m / z[\mathrm{M}+\mathrm{Na}]^{+} \mathrm{Calcd}$ for $\mathrm{C}_{19} \mathrm{H}_{22} \mathrm{O}_{4} \mathrm{~S}_{2} \mathrm{Na}$ 401.0857; found 401.0856.

\section{(R)-2-benzyl-2-(but-3-en-2-yl)malononitrile (3bb):}

White solid; Yield: $49 \mathrm{mg}, 93 \%$; M.P. $40-42{ }^{\circ} \mathrm{C}$.

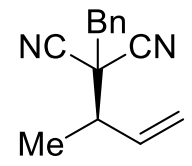

TLC R $\boldsymbol{f}=0.4$ (PE:EA, 9:1).

${ }^{1} \mathbf{H}$ NMR $\left(500 \mathrm{MHz}, \mathrm{CDCl}_{3}\right) \delta 7.41-7.38(\mathrm{~m}, 5 \mathrm{H}), 5.89$ (ddd, $\left.J=17.0,10.3,8.8 \mathrm{~Hz}, 1 \mathrm{H}\right), 5.40$ $(\mathrm{dd}, J=22.5,13.6 \mathrm{~Hz}, 2 \mathrm{H}), 3.20(\mathrm{~d}, J=13.7 \mathrm{~Hz}, 1 \mathrm{H}), 3.08(\mathrm{~d}, J=13.8 \mathrm{~Hz}, 1 \mathrm{H}), 2.77-2.71(\mathrm{~m}$,

$1 \mathrm{H}), 1.46(\mathrm{~d}, J=6.8 \mathrm{~Hz}, 3 \mathrm{H})$.

${ }^{13}$ C NMR $\left(126 \mathrm{MHz}, \mathrm{CDCl}_{3}\right) \delta$ 135.0, 132.6, 130.3, 129.1, 128.9, 121.0, 114.9, 114.4, 45.2, 44.9, 41.6, 17.2.

HPLC (Shimadzu LC-2030) (Daicel Chiralpak OJ-H Column, ${ }^{i} \mathrm{PrOH}: n-\mathrm{Hexane}=5.0: 95.0,1.0 \mathrm{~mL} / \mathrm{min}$ ), R $t=$ $9.75 \mathrm{~min}$ (major) and $15.05 \mathrm{~min}$ (minor), $90 \%$ ee.

$[\boldsymbol{\alpha}]_{D}^{25}=-22.0\left(c 0.05, \mathrm{CHCl}_{3}\right)$.

HRMS (ESI): $m / z$ [M + Na $]^{+}$Calcd for $\mathrm{C}_{14} \mathrm{H}_{14} \mathrm{~N}_{2} \mathrm{Na} 233.1055$; found 233.1054.

\section{(R)-2-benzyl-2-(hex-1-en-3-yl)malononitrile (3cb):}

White solid; Yield: $52 \mathrm{mg}, 87 \%$; M.P. $42-43^{\circ} \mathrm{C}$.

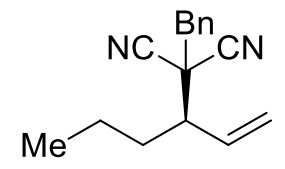

TLC R $\boldsymbol{f}=0.5$ (PE:EA, 9:1).

${ }^{1} \mathbf{H}$ NMR $\left(400 \mathrm{MHz}, \mathrm{CDCl}_{3}\right) \delta 7.43-7.37(\mathrm{~m}, 5 \mathrm{H}), 5.71(\mathrm{dt}, J=16.9,9.9 \mathrm{~Hz}, 1 \mathrm{H}), 5.49$ (dd, $J=10.2,1.2 \mathrm{~Hz}, 1 \mathrm{H}), 5.35(\mathrm{dd}, J=16.9,0.6 \mathrm{~Hz}, 1 \mathrm{H}), 3.23(\mathrm{~d}, J=13.7 \mathrm{~Hz}, 1 \mathrm{H}), 3.05$ $(\mathrm{d}, J=13.7 \mathrm{~Hz}, 1 \mathrm{H}), 2.54-2.48(\mathrm{~m}, 1 \mathrm{H}), 1.94-1.86(\mathrm{~m}, 1 \mathrm{H}), 1.71-1.65(\mathrm{~m}, 1 \mathrm{H}), 1.55-1.44(\mathrm{~m}, 1 \mathrm{H}), 1.34-$ $1.23(\mathrm{~m}, 1 \mathrm{H}), 0.96(\mathrm{t}, J=7.3 \mathrm{~Hz}, 3 \mathrm{H})$.

${ }^{13} \mathrm{C}$ NMR $\left(126 \mathrm{MHz}, \mathrm{CDCl}_{3}\right) \delta 133.7,132.6,130.4,129.1,128.8,122.8,115.2,114.5,50.9,44.6,41.9,33.1$, 20.3, 13.8 .

HPLC (Shimadzu LC-2030) (Daicel Chiralpak OD-H Column, ${ }^{i}$ PrOH: $n$-Hexane = 5.0:95.0, $1.0 \mathrm{~mL} / \mathrm{min}$ ), R $t=$ $6.27 \mathrm{~min}$ (minor) and $7.15 \mathrm{~min}$ (major), $99 \%$ ee.

$[\boldsymbol{\alpha}]_{D}^{25}=-29.9\left(c 0.05, \mathrm{CHCl}_{3}\right)$.

HRMS (ESI): $m / z[\mathrm{M}+\mathrm{Na}]^{+}$Calcd for $\mathrm{C}_{16} \mathrm{H}_{18} \mathrm{~N}_{2} \mathrm{Na}$ 261.1368; found 261.1368. 


\section{(R)-2-benzyl-2-(6-((tert-butyldimethylsilyl)oxy)hex-1-en-3-yl)malononitrile (3db):}

White solid; Yield: $68 \mathrm{mg}, 74 \%$; M.P. $43-44{ }^{\circ} \mathrm{C}$.

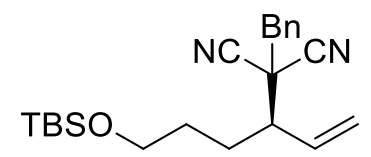

TLC R $f=0.7$ (PE:EA, 9:1).

${ }^{1} \mathbf{H}$ NMR $\left(400 \mathrm{MHz}, \mathrm{CDCl}_{3}\right) \delta 7.40(\mathrm{~s}, 5 \mathrm{H}), 5.76-5.67(\mathrm{~m}, 1 \mathrm{H}), 5.51(\mathrm{~d}, J=10.2 \mathrm{~Hz}$, $1 \mathrm{H}), 5.36(\mathrm{~d}, J=16.9 \mathrm{~Hz}, 1 \mathrm{H}), 3.65(\mathrm{t}, J=6.0 \mathrm{~Hz}, 2 \mathrm{H}), 3.23(\mathrm{~d}, J=13.7 \mathrm{~Hz}, 1 \mathrm{H})$,

$3.06(\mathrm{~d}, J=13.8 \mathrm{~Hz}, 1 \mathrm{H}), 2.54(\mathrm{t}, J=10.2 \mathrm{~Hz}, 1 \mathrm{H}), 2.11-2.04(\mathrm{~m}, 1 \mathrm{H}), 1.74-1.64(\mathrm{~m}, 2 \mathrm{H}), 1.55-1.44(\mathrm{~m}$, 1H), 0.91 (s, 9H), 0.06 (s, 6H).

${ }^{13} \mathrm{C}$ NMR $\left(126 \mathrm{MHz}, \mathrm{CDCl}_{3}\right) \delta 133.7,132.6,130.4,129.1,128.8,123.0,115.0,114.5,62.5,50.8,44.6,41.9$, $30.2,27.5,26.1,18.5,-5.1$.

HPLC (Shimadzu LC-2030) (Daicel Chiralpak AD-H Column, ${ }^{i} \mathrm{PrOH}: n-H e x a n e=2.0: 98.0,1.0 \mathrm{~mL} / \mathrm{min}$ ), R $t=$ $10.40 \mathrm{~min}$ (major) and $11.84 \mathrm{~min}$ (minor), $99 \%$ ee.

$[\boldsymbol{\alpha}]_{D}^{\mathbf{2 5}}=-15.3\left(c 0.05, \mathrm{CHCl}_{3}\right)$.

HRMS (ESI): $m / z[\mathrm{M}+\mathrm{H}]^{+}$Calcd for $\mathrm{C}_{22} \mathrm{H}_{33} \mathrm{~N}_{2} \mathrm{OSi}$ 369.2357; found 369.2361; and $[\mathrm{M}+\mathrm{Na}]^{+}$Calcd for $\mathrm{C}_{22} \mathrm{H}_{32} \mathrm{~N}_{2} \mathrm{OSiNa}$ 391.2182; found 391.2168.

(R)-2-benzyl-2-(5-methylhex-1-en-3-yl)malononitrile (3eb):

White solid; Yield: $55.5 \mathrm{mg}, 88 \%$; M.P. $80-81{ }^{\circ} \mathrm{C}$.

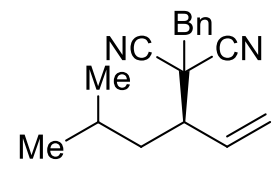

TLC R $\boldsymbol{f}=0.6$ (PE:EA, 9:1).

${ }^{1}$ H NMR $\left(500 \mathrm{MHz}, \mathrm{CDCl}_{3}\right) \delta 7.40(\mathrm{~s}, 5 \mathrm{H}), 5.74-5.66(\mathrm{~m}, 1 \mathrm{H}), 5.49(\mathrm{~d}, J=10.2 \mathrm{~Hz}, 1 \mathrm{H})$, $5.36(\mathrm{~d}, J=17.0 \mathrm{~Hz}, 1 \mathrm{H}), 3.22(\mathrm{~d}, J=13.7 \mathrm{~Hz}, 1 \mathrm{H}), 3.04(\mathrm{~d}, J=13.8 \mathrm{~Hz}, 1 \mathrm{H}), 2.60(\mathrm{t}, J=$ $10.5 \mathrm{~Hz}, 1 \mathrm{H}), 1.74-1.60(\mathrm{~m}, 3 \mathrm{H}), 0.99$ (d, $J=5.6 \mathrm{~Hz}, 3 \mathrm{H}), 0.89$ (d, $J=5.6 \mathrm{~Hz}, 3 \mathrm{H})$.

${ }^{13} \mathbf{C}$ NMR $\left(126 \mathrm{MHz}, \mathrm{CDCl}_{3}\right) \delta 133.8,132.7,130.4,129.1,128.8,122.7,115.2,114.5,49.4,44.8,41.9,40.0$, 25.3, 24.0, 20.7.

HPLC (Shimadzu LC-2030) (Daicel Chiralpak OD-H Column, ${ }^{i} \mathrm{PrOH}: n-\mathrm{Hexane}=5.0: 95.0,1.0 \mathrm{~mL} / \mathrm{min}$ ), R $t=$ $5.25 \mathrm{~min}$ (minor) and $6.28 \mathrm{~min}$ (major), > 99\% ee.

$[\alpha]_{D}^{25}=-14.5\left(c 0.05, \mathrm{CHCl}_{3}\right)$.

HRMS (ESI): $m / z[\mathrm{M}+\mathrm{Na}]^{+}$Calcd for $\mathrm{C}_{17} \mathrm{H}_{20} \mathrm{~N}_{2} \mathrm{Na} 275.1524$; found 275.1525 .

\section{(S)-2-benzyl-2-(1-cyclopropylallyl)malononitrile (3fb):}

White solid; Yield: $56 \mathrm{mg}$, 95\%; M.P. $51-53{ }^{\circ} \mathrm{C}$.

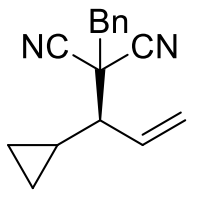

TLC R $\boldsymbol{f}=0.4$ (PE:EA, 9:1).

${ }^{1} \mathbf{H}$ NMR $\left(400 \mathrm{MHz}, \mathrm{CDCl}_{3}\right) \delta 7.42-7.36(\mathrm{~m}, 5 \mathrm{H}), 5.95-5.86(\mathrm{~m}, 1 \mathrm{H}), 5.45(\mathrm{dd}, J=10.3,0.9$ $\mathrm{Hz}, 1 \mathrm{H}), 5.33(\mathrm{~d}, J=16.9 \mathrm{~Hz}, 1 \mathrm{H}), 3.24-3.12(\mathrm{~m}, 2 \mathrm{H}), 2.00(\mathrm{t}, J=9.0 \mathrm{~Hz}, 1 \mathrm{H}), 1.21-1.13(\mathrm{~m}$, $1 \mathrm{H}), 0.89-0.83(\mathrm{~m}, 1 \mathrm{H}), 0.70-0.58(\mathrm{~m}, 2 \mathrm{H}), 0.32-0.25(\mathrm{~m}, 1 \mathrm{H})$. 
${ }^{13} \mathrm{C}$ NMR $\left(126 \mathrm{MHz}, \mathrm{CDCl}_{3}\right) \delta 133.1,132.7,130.4,129.0,128.8,121.7,114.9,114.8,54.8,44.8,42.0,12.6$, 6.7, 3.1.

HPLC (Shimadzu LC-2030) (Daicel Chiralpak OD-H Column, ${ }^{i}$ PrOH: $n$-Hexane = 5.0:95.0, $1.0 \mathrm{~mL} / \mathrm{min}$ ), R $t=$ $9.04 \mathrm{~min}$ (minor) and $9.74 \mathrm{~min}$ (major), $99 \%$ ee.

$[\alpha]_{D}^{25}=-11.5\left(c 0.05, \mathrm{CHCl}_{3}\right)$.

HRMS (ESI): $m / z[\mathrm{M}+\mathrm{Na}]^{+}$Calcd for $\mathrm{C}_{16} \mathrm{H}_{16} \mathrm{~N}_{2} \mathrm{Na}$ 259.1211; found 259.1211.

\section{(R)-2-benzyl-2-(4-methylpent-1-en-3-yl)malononitrile (3gb):}

White solid; Yield: $40 \mathrm{mg}, 67 \%$; M.P. $82-83^{\circ} \mathrm{C}$.

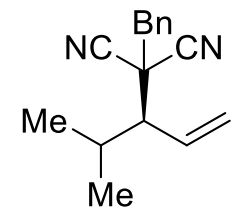

TLC R $\boldsymbol{f}=0.4$ (PE:EA, 9:1).

${ }^{1} \mathbf{H}$ NMR $\left(400 \mathrm{MHz}, \mathrm{CDCl}_{3}\right) \delta 7.39(\mathrm{~s}, 5 \mathrm{H}), 5.89(\mathrm{dt}, J=16.8,10.1 \mathrm{~Hz}, 1 \mathrm{H}), 5.56(\mathrm{dd}, J=10.2$, $1.4 \mathrm{~Hz}, 1 \mathrm{H}), 5.34(\mathrm{~d}, J=16.9 \mathrm{~Hz}, 1 \mathrm{H}), 3.27(\mathrm{~d}, J=13.7 \mathrm{~Hz}, 1 \mathrm{H}), 3.03(\mathrm{~d}, J=13.7 \mathrm{~Hz}, 1 \mathrm{H})$, $2.38(\mathrm{dd}, J=7.3,3.7 \mathrm{~Hz}, 2 \mathrm{H}), 1.12(\mathrm{~d}, J=6.6 \mathrm{~Hz}, 3 \mathrm{H}), 1.00(\mathrm{~d}, J=6.5 \mathrm{~Hz}, 3 \mathrm{H})$.

${ }^{13} \mathrm{C} \mathrm{NMR}\left(126 \mathrm{MHz}, \mathrm{CDCl}_{3}\right) \delta 132.5,130.6,130.5,129.0,128.9,124.1,115.1,115.0,55.9,43.4,42.6,30.4$, 22.7, 17.1.

HPLC (Shimadzu LC-2030) (Daicel Chiralpak OD-H Column, ${ }^{i} \operatorname{PrOH}: n-H e x a n e=5.0: 95.0,1.0 \mathrm{~mL} / \mathrm{min}$ ), R $t=$ $6.07 \mathrm{~min}$ (minor) and $7.83 \mathrm{~min}$ (major), $98 \%$ ee.

$[\boldsymbol{\alpha}]_{D}^{25}=-11.5\left(c 0.05, \mathrm{CHCl}_{3}\right)$.

HRMS (ESI): $m / z[\mathrm{M}+\mathrm{Na}]^{+} \mathrm{Calcd}$ for $\mathrm{C}_{16} \mathrm{H}_{18} \mathrm{~N}_{2} \mathrm{Na} 261.1368$; found 261.1363.

\section{(S)-2-benzyl-2-(1-cyclohexylallyl)malononitrile (3hb):}

White solid; Yield: $49 \mathrm{mg}, 70 \%$; M.P. $130-132{ }^{\circ} \mathrm{C}$.

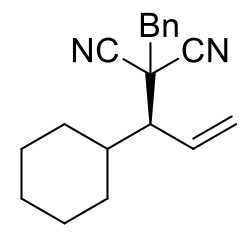

TLC R $\boldsymbol{f}=0.5$ (PE:EA, 9:1).

${ }^{1} \mathbf{H}$ NMR $\left(500 \mathrm{MHz}, \mathrm{CDCl}_{3}\right) \delta 7.42-7.37(\mathrm{~m}, 5 \mathrm{H}), 5.94-5.87(\mathrm{~m}, 1 \mathrm{H}), 5.52(\mathrm{dd}, J=10.2$, $1.4 \mathrm{~Hz}, 1 \mathrm{H}), 5.31(\mathrm{dd}, J=16.9,1.1 \mathrm{~Hz}, 1 \mathrm{H}), 3.26(\mathrm{~d}, J=13.7 \mathrm{~Hz}, 1 \mathrm{H}), 3.02(\mathrm{~d}, J=13.7 \mathrm{~Hz}$, 1H), $2.34(\mathrm{dd}, J=10.2,3.4 \mathrm{~Hz}, 1 \mathrm{H}), 2.11(\mathrm{~d}, J=12.7 \mathrm{~Hz}, 1 \mathrm{H}), 2.01-1.95(\mathrm{~m}, 1 \mathrm{H}), 1.82-$ $1.75(\mathrm{~m}, 2 \mathrm{H}), 1.68(\mathrm{~d}, J=13.0 \mathrm{~Hz}, 1 \mathrm{H}), 1.39-1.06(\mathrm{~m}, 6 \mathrm{H})$.

${ }^{13} \mathrm{C}$ NMR $\left(126 \mathrm{MHz}, \mathrm{CDCl}_{3}\right) \delta 132.5,131.7,130.6,129.0,128.9,123.5,115.1,115.1,56.2,43.4,42.3,40.5$, $32.8,28.0,26.5,26.3,26.2$.

HPLC (Shimadzu LC-2030) (Daicel Chiralpak OD-H Column, ${ }^{i}$ PrOH: $n$-Hexane = 5.0:95.0, $1.0 \mathrm{~mL} / \mathrm{min}$ ), R $t=$ $5.68 \mathrm{~min}$ (minor) and $6.77 \mathrm{~min}$ (major), > 99\% ee.

$[\boldsymbol{\alpha}]_{\boldsymbol{D}}^{25}=-20.3\left(c 0.05, \mathrm{CHCl}_{3}\right)$.

HRMS (ESI): $m / z[\mathrm{M}+\mathrm{Na}]^{+} \mathrm{Calcd}$ for $\mathrm{C}_{19} \mathrm{H}_{22} \mathrm{~N}_{2} \mathrm{Na} 301.1681$; found 301.1669. 


\section{(S)-2-benzyl-2-(1-phenylallyl)malononitrile (3ib):}

White solid; Yield: $60.5 \mathrm{mg}, 89 \%$; M.P. $73-74{ }^{\circ} \mathrm{C}$.<smiles>C=CC(c1ccccc1)C(Br)(C#N)C#N</smiles>

TLC Rf $=0.3$ (PE:EA, 9:1).

${ }^{1} \mathbf{H}$ NMR $\left(400 \mathrm{MHz}, \mathrm{CDCl}_{3}\right) \delta 7.48-7.35(\mathrm{~m}, 10 \mathrm{H}), 6.40(\mathrm{ddd}, J=16.8,10.0,9.3 \mathrm{~Hz}, 1 \mathrm{H})$, $5.52(\mathrm{~d}, J=10.2 \mathrm{~Hz}, 1 \mathrm{H}), 5.46(\mathrm{~d}, J=16.8 \mathrm{~Hz}, 1 \mathrm{H}), 3.75(\mathrm{~d}, J=9.2 \mathrm{~Hz}, 1 \mathrm{H}), 3.19(\mathrm{~d}, J=13.7$ $\mathrm{Hz}, 1 \mathrm{H}), 2.98(\mathrm{~d}, J=13.7 \mathrm{~Hz}, 1 \mathrm{H})$.

${ }^{13}$ C NMR $\left(126 \mathrm{MHz}, \mathrm{CDCl}_{3}\right) \delta 136.1,133.3,132.4,130.4,129.4,129.2,129.1,128.9,122.2,114.9,114.5,56.5$, 45.6, 42.5.

HPLC (Shimadzu LC-2030) (Daicel Chiralpak OD-H Column, ${ }^{i} \mathrm{PrOH}: n-\mathrm{Hexane}=5.0: 95.0,1.0 \mathrm{~mL} / \mathrm{min}$ ), R $t=$ $9.23 \mathrm{~min}$ (minor) and $10.30 \mathrm{~min}$ (major), $99 \%$ ee.

$[\boldsymbol{\alpha}]_{D}^{25}=+19.8\left(c 0.05, \mathrm{CHCl}_{3}\right)$.

HRMS (ESI): $m / z$ [M + Na] $]^{+}$Calcd for $\mathrm{C}_{19} \mathrm{H}_{16} \mathrm{~N}_{2} \mathrm{Na} 295.1211$; found 295.1211.

\section{Synthesis of (R)-2-(1-phenylallyl)malononitrile (3ja): ${ }^{6}$}

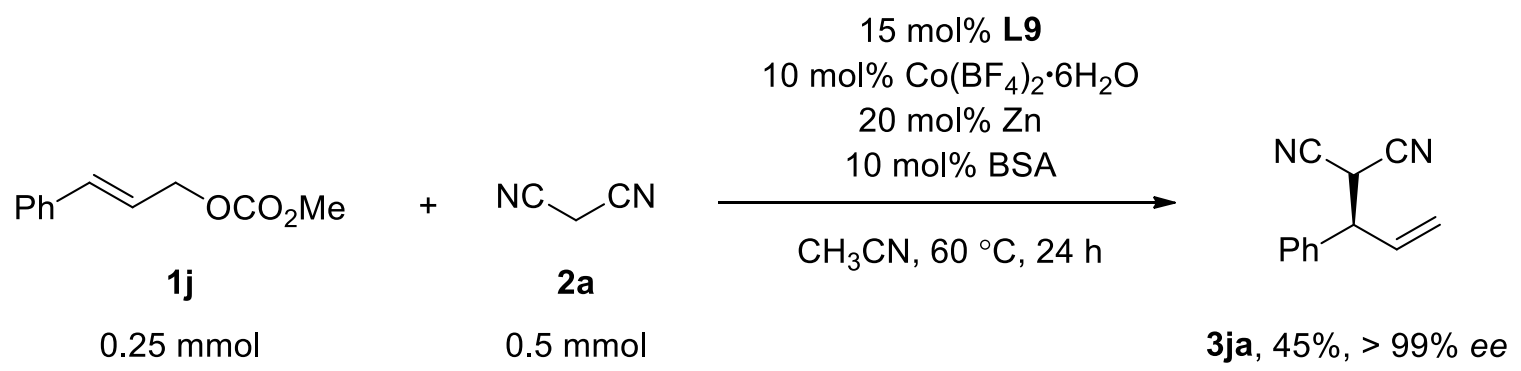

In an $\mathrm{N}_{2}$ filled glove box, $\mathbf{L 9}\left(16 \mathrm{mg}, 37.5 \times 10^{-3} \mathrm{mmol}, 15 \mathrm{~mol} \%\right)$ and $\mathrm{Co}\left(\mathrm{BF}_{4}\right)_{2} \cdot 6 \mathrm{H}_{2} \mathrm{O}\left(8.6 \mathrm{mg}, 25.2 \times 10^{-3} \mathrm{mmol}\right.$, $10 \mathrm{~mol} \%$ ) in $0.2 \mathrm{~mL}$ acetonitrile was taken into a $10 \mathrm{~mL}$ reaction tube equipped with a Teflon stopcock and a stir bar. $\mathrm{Zn}$ dust $\left(3.3 \mathrm{mg}, 50.5 \times 10^{-3} \mathrm{mmol}, 20 \mathrm{~mol} \%\right)$ and $0.5 \mathrm{~mL}$ acetonitrile to complete transfer of zinc were added to it. The mixture was allowed to stir for 20 minutes. Afterward, (E)-cinnamyl methyl carbonate (1j) (48.2 $\mathrm{mg}, 0.25 \mathrm{mmol}, 1 \mathrm{eq}$ ), malonitrile (2a) $(33 \mathrm{mg}, 0.5 \mathrm{mmol}, 2 \mathrm{eq})$, bis(trimethylsilyl)acetamide (BSA) (6 $\mu \mathrm{L}$, $25 \times 10^{-3} \mathrm{mmol}, 10 \mathrm{~mol} \%$ ), and $0.3 \mathrm{~mL}$ of acetonitrile were added sequentially to the reaction mixture. The reaction tube was sealed and brought out from the glove box. The reaction mixture was then stirred for 24 hours at $60{ }^{\circ} \mathrm{C}$ in oil bath. Then, it was cooled to room temperature. and solvent was removed under reduced pressure. The desired pure product was obtained by silica gels (200-300 mesh) column chromatography using PE/EA as an eluent.

Clear oil; Yield: $20.5 \mathrm{mg}, 45 \%$.

TLC R $\boldsymbol{f}=0.2$ (PE:EA, 9:1).

${ }^{1}$ H NMR $\left(400 \mathrm{MHz}, \mathrm{CDCl}_{3}\right) \delta 7.45-7.32(\mathrm{~m}, 5 \mathrm{H}), 6.17$ (ddd, $\left.J=17.0,10.3,7.7 \mathrm{~Hz}, 1 \mathrm{H}\right), 5.49$ (d, $J=10.3 \mathrm{~Hz}$, $1 \mathrm{H}), 5.43(\mathrm{dd}, J=17.0,0.8 \mathrm{~Hz}, 1 \mathrm{H}), 4.01(\mathrm{~d}, J=6.6 \mathrm{~Hz}, 1 \mathrm{H}), 3.94(\mathrm{t}, J=7.2 \mathrm{~Hz}, 1 \mathrm{H})$. 
${ }^{13} \mathbf{C}$ NMR $\left(126 \mathrm{MHz}, \mathrm{CDCl}_{3}\right) \delta 136.3,133.3,129.6,129.1,128.0,121.1,111.8,111.8,50.3,29.9$.

HPLC (Shimadzu LC-16) (Daicel Chiralpak OD-H Column, ${ }^{i} \mathrm{PrOH}: n-\mathrm{Hexane}=10.0: 90.0,1.0 \mathrm{~mL} / \mathrm{min}$ ), R $t=$ $17.01 \mathrm{~min}$ (minor) and $22.55 \mathrm{~min}$ (major), > 99\% ee.

$[\boldsymbol{\alpha}]_{D}^{25}=+6.1\left(c 0.05, \mathrm{CHCl}_{3}\right)$. Literature report: $[\boldsymbol{\alpha}]_{\boldsymbol{D}}^{\mathbf{2 4}}=+41.4\left(c 0.54, \mathrm{CHCl}_{3}\right), 91 \%$ ee.

HRMS (ESI): $m / z[\mathrm{M}+\mathrm{K}]^{+}$calculated for $\mathrm{C}_{12} \mathrm{H}_{10} \mathrm{~N}_{2} \mathrm{~K} 221.0481$; found 221.0396.

\section{Kinetic resolution study:}

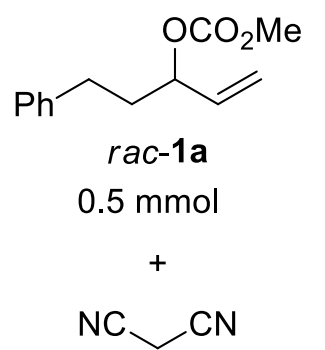

$2 a$

$0.25 \mathrm{mmol}$
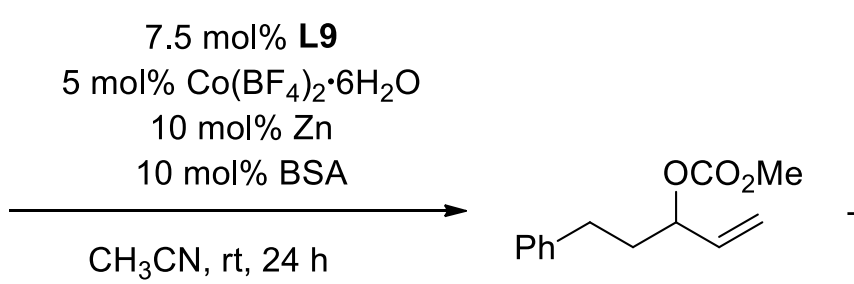

$1 \mathrm{a}, 64 \%$ recovery $8 \%$ ee

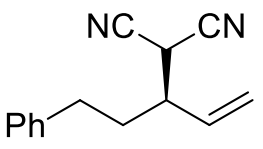

3aa, $59 \%$, 99\% ee

In an $\mathrm{N}_{2}$ filled glove box, a reaction tube contains $\mathbf{L 9}\left(8 \mathrm{mg}, 18.8 \times 10^{-3} \mathrm{mmol}, 7.5 \mathrm{~mol} \%\right)$ and $\mathrm{Co}\left(\mathrm{BF}_{4}\right)_{2} \cdot 6 \mathrm{H}_{2} \mathrm{O}$ (4.3 mg, $12.6 \times 10^{-3} \mathrm{mmol}, 5 \mathrm{~mol} \%$ ) in $0.2 \mathrm{~mL}$ acetonitrile was added $\mathrm{Zn}$ dust $\left(1.7 \mathrm{mg}, 26.0 \times 10^{-3} \mathrm{mmol}, 10 \mathrm{~mol} \%\right.$ ). Additionally, $0.5 \mathrm{~mL}$ of acetonitrile was added to complete transfer of zinc. The mixture was allowed to stir for 20 minutes. Afterward, rac-1a (110 mg, $0.5 \mathrm{mmol}, 2 \mathrm{eq}$ ), malonitrile (2a) (17 mg, $0.25 \mathrm{mmol}, 1 \mathrm{eq})$, bis(trimethylsilyl)acetamide (BSA) $\left(6 \mu \mathrm{L}, 25 \times 10^{-3} \mathrm{mmol}, 10 \mathrm{~mol} \%\right)$, and $0.3 \mathrm{~mL}$ of acetonitrile were added sequentially to the reaction mixture. The reaction tube was sealed with Teflon stopcock and brought out from the glove box. The reaction mixture was then stirred for 16 hours at room temperature. Then, solvent was removed and column chromatography was run on a column of silica gels (200-300 mesh) using PE/EA as an eluent to get the desired product.

The amount of recovery $1 \mathrm{a}$ was $70 \mathrm{mg}$, 64\% yield; ee $8 \%$.

The amount of product 3aa was $31 \mathrm{mg}$, 30\% yield; ee $99 \%$.

Enantiomeric excess of recovered 1a was determined by HPLC (Shimadzu LC-2030) (Daicel Chiralpak OD-H Column, ${ }^{i} \mathrm{PrOH}: n-\mathrm{Hexane}=5.0: 95.0,1.0 \mathrm{~mL} / \mathrm{min}$ ), $\mathrm{R} t=4.65 \mathrm{~min}$ (minor) and $4.92 \mathrm{~min}$ (major), $6 \%$ ee. 


\section{Synthesis of $(R)-2-p h e n e t h y l b u t-3-e n-1-o l(4)$ :}

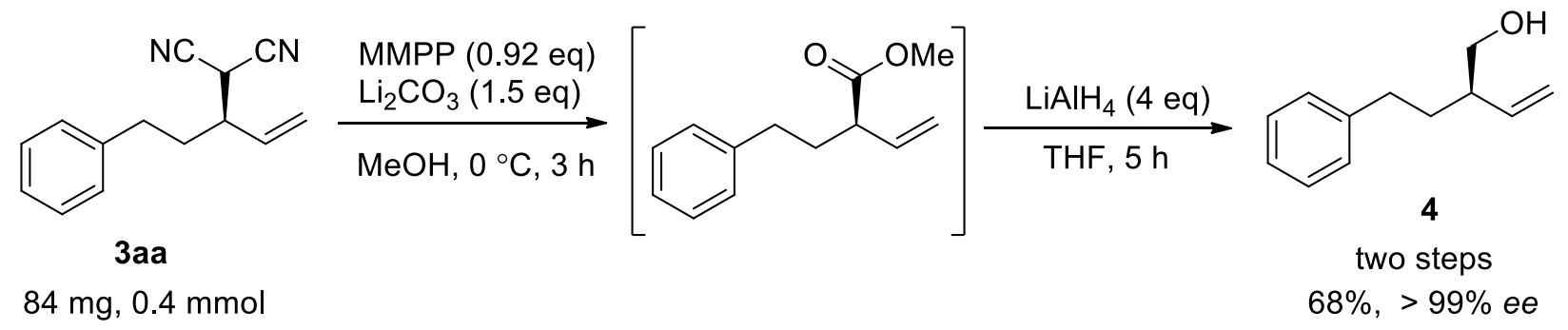

Compound 3aa ( $84 \mathrm{mg}, 0.4 \mathrm{mmol})$ was dissolved in $\mathrm{MeOH}(3 \mathrm{~mL})$ in a $10 \mathrm{~mL}$ round bottom flask at $0{ }^{\circ} \mathrm{C}$. $\mathrm{Li}_{2} \mathrm{CO}_{3}$ (45 mg, $0.6 \mathrm{mmol}, 1.5 \mathrm{eq}$ ) and Magnesium monoperoxyphthalate hexahydrate (MMPP) $80 \%$ analytical grade (144 mg, $0.92 \mathrm{eq}$ ) were added sequentially to it. The reaction mixture was stirred for 3 hours at $0{ }^{\circ} \mathrm{C}$. Then, it was diluted with EA $(20 \mathrm{~mL})$ and filtered through a pad of celite to remove solid residues and concentrated in vacuo. The crude product was further dissolved in EA $(20 \mathrm{~mL})$ and filtered through a pad of celite to get the clear oily product. The crude oily product was treated with $\mathrm{LiAlH}_{4}(66 \mathrm{mg}, 4 \mathrm{eq})$ in $8 \mathrm{~mL}$ THF at $0{ }^{\circ} \mathrm{C}$ and stirred continue at room temperature for 5 hours. The reaction mixture was quenched with water $(5 \mathrm{~mL})$ carefully and $5 \mathrm{~mL}$ of dil. $\mathrm{HCl}(1 \mathrm{M})$ was added and extracted with EA. The organic phase was dried on $\mathrm{Na}_{2} \mathrm{SO}_{4}$ and chromatographed on a column of silica gels (PE/EA as an eluent).

Clear oil; Yield: 48 mg, 68\%.

TLC $\mathbf{R} f=0.3$ (PE:EA, 4:1).

${ }^{1}$ H NMR (400 MHz, $\left.\mathrm{CDCl}_{3}\right) \delta 7.32-7.28(\mathrm{~m}, 2 \mathrm{H}), 7.22-7.18(\mathrm{~m}, 3 \mathrm{H}), 5.66(\mathrm{ddd}, J=17.2,10.3,8.8 \mathrm{~Hz}, 1 \mathrm{H})$, $5.22(\mathrm{ddd}, J=18.3,13.8,1.4 \mathrm{~Hz}, 2 \mathrm{H}), 3.59$ (dd, $J=10.6,5.2 \mathrm{~Hz}, 1 \mathrm{H}), 3.47$ (dd, $J=10.6,8.0 \mathrm{~Hz}, 1 \mathrm{H}), 2.72$ (ddd, $J=15.0,10.0,5.3 \mathrm{~Hz}, 1 \mathrm{H}), 2.58(\mathrm{ddd}, J=13.8,9.8,6.9 \mathrm{~Hz}, 1 \mathrm{H}), 2.32-2.23(\mathrm{~m}, 1 \mathrm{H}), 1.82-1.73(\mathrm{~m}, 1 \mathrm{H}), 1.67$ (s, $1 \mathrm{H}), 1.64-1.55(\mathrm{~m}, 1 \mathrm{H})$.

${ }^{13} \mathbf{C}$ NMR $\left(101 \mathrm{MHz}, \mathrm{CDCl}_{3}\right) \delta 142.3,139.8,128.6,128.5,125.9,118.0,65.7,46.6,33.4,32.6$.

$[\alpha]_{D}^{25}=+5.6\left(c 0.25, \mathrm{CHCl}_{3}\right)$.

HPLC (Shimadzu LC-2030) (Daicel Chiralpak OJ-H Column, ${ }^{i} \mathrm{PrOH}: n-\mathrm{Hexane}=1.0: 99.0,1.0 \mathrm{~mL} / \mathrm{min}$ ), R $t=$ $22.97 \mathrm{~min}$ (minor) and $24.04 \mathrm{~min}$ (major), > 99\% ee.

HRMS (ESI): $m / z$ [M + Na $]^{+}$Calcd for $\mathrm{C}_{12} \mathrm{H}_{16} \mathrm{ONa}$ 199.1099; found 199.1096. 


\section{Synthesis $(R)-2-p h e n e t h y l c y c l o p e n t-3-e n e-1,1-d i c a r b o n i t r i l e(5):$}<smiles>C=CCC(C)(C#N)C(C=C)CCc1ccccc1</smiles>

3ai

$53 \mathrm{mg}, 0.21 \mathrm{mmol}$

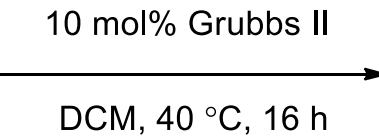

$40^{\circ} \mathrm{C}, 16 \mathrm{~h}$

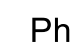

5

$77 \%,>99 \%$ ee

In a Schlenk tube equipped with Teflon stop cock and stir bar, 3ai (53 mg, $0.21 \mathrm{mmol}$ ) and Grubbs II (18 mg, $0.021 \mathrm{mmol}, 10 \mathrm{~mol} \%)$ were mixed in DCM $(5 \mathrm{~mL})$ under argon atmosphere. The reaction mixture was heated at $40{ }^{\circ} \mathrm{C}$ in oil bath for 16 hours. Then solvent was removed and column chromatography was performed on silica gels using PE/EA as an eluent.

Clear oil; Yield: $36 \mathrm{mg}, 77 \%$.

TLC R $\boldsymbol{f}=0.3$ (PE:EA, 20:1).

${ }^{1} \mathbf{H}$ NMR $\left(400 \mathrm{MHz}, \mathrm{CDCl}_{3}\right) \delta 7.33-7.30(\mathrm{~m}, 2 \mathrm{H}), 7.24-7.21(\mathrm{~m}, 3 \mathrm{H}), 5.82-5.76(\mathrm{~m}, 2 \mathrm{H}), 3.31$ (ddd, $J=8.4$, $3.7,1.8 \mathrm{~Hz}, 1 \mathrm{H}), 3.24-3.12(\mathrm{~m}, 2 \mathrm{H}), 2.81(\mathrm{dd}, J=11.7,4.8 \mathrm{~Hz}, 2 \mathrm{H}), 2.15(\mathrm{tt}, J=14.0,7.2 \mathrm{~Hz}, 1 \mathrm{H}), 2.00(\mathrm{ddd}$, $J=12.2,7.9,6.2 \mathrm{~Hz}, 1 \mathrm{H})$.

${ }^{13} \mathrm{C}$ NMR $\left(126 \mathrm{MHz}, \mathrm{CDCl}_{3}\right) \delta 140.3,132.4,128.9,128.5,127.2,126.6,116.9,115.0,54.7,44.9,37.9,33.6$.

HPLC (Shimadzu LC-16) (Daicel Chiralpak AD-H Column, ${ }^{i} \mathrm{PrOH}: n-\mathrm{Hexane}=2.0: 98.0,0.5 \mathrm{~mL} / \mathrm{min}$ ), R $t=$ $16.92 \mathrm{~min}$ (minor) and $18.11 \mathrm{~min}$ (major), > 99\% ee.

$[\alpha]_{D}^{25}=-92.8\left(c 0.25, \mathrm{CHCl}_{3}\right)$.

HRMS (ESI): $m / z$ [M + Na] ${ }^{+}$Calcd for $\mathrm{C}_{15} \mathrm{H}_{14} \mathrm{~N}_{2} \mathrm{Na}$ 245.1055; found 245.1059.

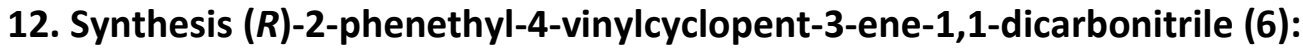

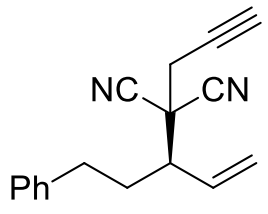

3aj

$0.124 \mathrm{~g}, 0.5 \mathrm{mmol}$

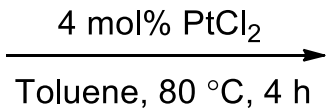

Toluene, $80^{\circ} \mathrm{C}, 4 \mathrm{~h}$

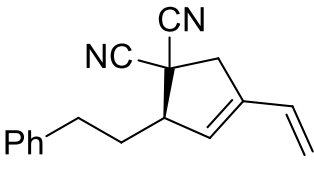

6

Under Argon atmosphere, a Schlenk tube equipped with Teflon stop cock and stir bar was charged with 3aj $(0.124 \mathrm{~g}, 0.5 \mathrm{mmol})$ and $\mathrm{PtCl}_{2}(5.5 \mathrm{mg}, 0.02 \mathrm{mmol}, 4 \mathrm{~mol} \%)$ in Toluene $(5 \mathrm{~mL})$. The reaction mixture was heated 
at $80^{\circ} \mathrm{C}$ in oil bath for 4 hours. Then, solvent was removed and column chromatography was performed on silica gels using PE/EA as an eluent.

Clear oil; Yield: $109 \mathrm{mg}, 88 \%$.

TLC R $f=0.3$ (PE:EA, 4:1).

${ }^{1} \mathbf{H}$ NMR $\left(400 \mathrm{MHz}, \mathrm{CDCl}_{3}\right) \delta 7.33-7.29(\mathrm{~m}, 2 \mathrm{H}), 7.24-7.21(\mathrm{~m}, 3 \mathrm{H}), 6.47(\mathrm{dd}, J=17.6,10.8 \mathrm{~Hz}, 1 \mathrm{H}), 5.67$ (s, 1H), $5.27(\mathrm{~d}, J=10.8 \mathrm{~Hz}, 1 \mathrm{H}), 5.15(\mathrm{~d}, J=17.6 \mathrm{~Hz}, 1 \mathrm{H}), 3.37(\mathrm{t}, J=7.7 \mathrm{~Hz}, 1 \mathrm{H}), 3.34-3.19(\mathrm{~m}, 2 \mathrm{H}), 2.81$ (t, $J=7.9 \mathrm{~Hz}, 2 \mathrm{H}), 2.17(\mathrm{dt}, J=14.5,7.8 \mathrm{~Hz}, 1 \mathrm{H}), 2.00(\mathrm{dq}, J=13.7,8.1 \mathrm{~Hz}, 1 \mathrm{H})$.

${ }^{13} \mathbf{C}$ NMR $\left(126 \mathrm{MHz}, \mathrm{CDCl}_{3}\right) \delta 140.3,139.1,131.0,129.3,128.9,128.5,126.6,118.2,116.7,114.9,54.4,43.2$, $37.9,33.7,33.5$.

$[\alpha]_{D}^{25}=-133.2\left(c 0.25, \mathrm{CHCl}_{3}\right)$.

HPLC (Shimadzu LC-2030) (Daicel Chiralpak OD-H Column, ${ }^{i} \mathrm{PrOH}: n-\mathrm{Hexane}=1.0: 99.0,0.5 \mathrm{~mL} / \mathrm{min}$ ), R $t$ $=45.73 \mathrm{~min}$ (minor) and $47.24 \mathrm{~min}$ (major), $>99 \%$ ee

HRMS (ESI): $m / z$ [M + Na] $]^{+}$Calcd for $\mathrm{C}_{17} \mathrm{H}_{16} \mathrm{~N}_{2} \mathrm{Na} 271.1211$; found 271.1215.

\section{Synthesis (R)-5-(5-phenylpent-1-en-3-yl)pyrimidine-2,4,6-triamine (7):}

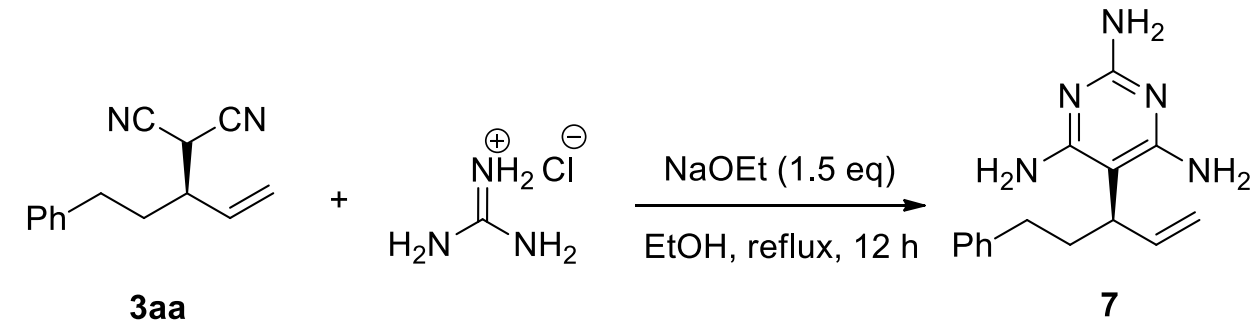

$63 \mathrm{mg}, 0.3 \mathrm{mmol} \quad 36 \mathrm{mg}, 0.38 \mathrm{mmol} \quad$ Yield: $82 \%$

To an ethanolic solution of 3aa (63 mg, $0.3 \mathrm{mmol})$ and guanidine hydrochloride (36 mg, $0.38 \mathrm{mmol}, 1.25 \mathrm{eq})$, was added NaOEt (32 mg, $0.45 \mathrm{mmol}, 1.5 \mathrm{eq}$ ) and the reaction mixture was refluxed in oil bath for 12 hours. Then, solvent was removed and column chromatography was performed on silica gels using DCM/MeOH as an eluent.

Light yellow solid; Yield: $66 \mathrm{mg}, 82 \%$; M.P. $63-64{ }^{\circ} \mathrm{C}$.

TLC R $\boldsymbol{f}=0.3$ (PE:EA, 4:1).

${ }^{1}$ H NMR (400 MHz, MeOD) $\delta 7.21$ (dd, $\left.J=10.1,4.7 \mathrm{~Hz}, 2 \mathrm{H}\right), 7.14-7.10(\mathrm{~m}, 3 \mathrm{H}), 6.07-5.99$ (m, 1H), 5.17 $(\mathrm{d}, J=2.3 \mathrm{~Hz}, 1 \mathrm{H}), 5.13(\mathrm{dt}, J=3.6,1.5 \mathrm{~Hz}, 1 \mathrm{H}), 4.93(\mathrm{~s}, 6 \mathrm{H}), 3.46(\mathrm{dtt}, J=11.1,4.4,2.3 \mathrm{~Hz}, 1 \mathrm{H}), 2.64-2.48$ $(\mathrm{m}, 2 \mathrm{H}), 2.10-2.04(\mathrm{~m}, 2 \mathrm{H})$.

${ }^{13}$ C NMR (126 MHz, MeOD) $\delta$ 163.5, 161.4, 143.8, 141.0, 129.5, 129.5, 126.9, 115.1, 88.0, 39.1, 35.2, 33.8 . $[\alpha]_{D}^{25}=-35.6(c 0.25, \mathrm{MeOH})$.

HRMS (ESI): $m / z,[\mathrm{M}+\mathrm{H}]^{+}$Calcd for $\mathrm{C}_{15} \mathrm{H}_{20} \mathrm{~N}_{5}$ 270.1713; found 270.1714. 


\section{NMR spectra of new compounds:}

$500 \mathrm{MHz}, \mathrm{CDCl}_{3}$

$$
\text { [LS1] }
$$

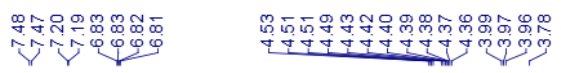<smiles>COc1ccc(Br)c(C2=NC(C)CO2)c1</smiles>
LS1

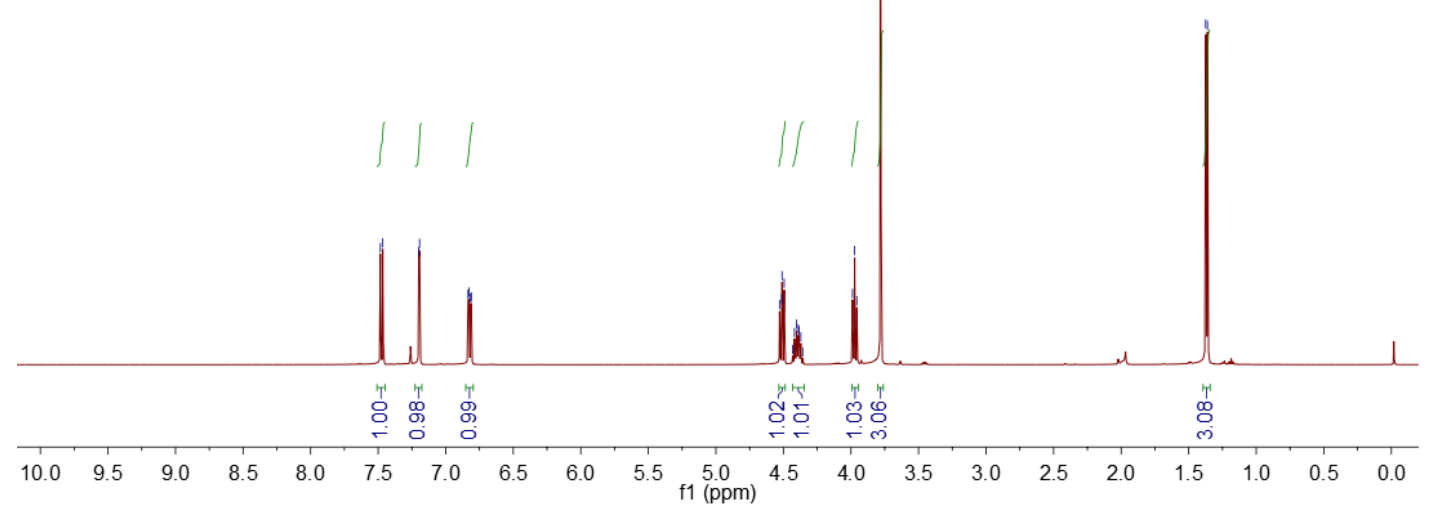

$126 \mathrm{MHz}, \mathrm{CDCl}_{3}$

[LS1]

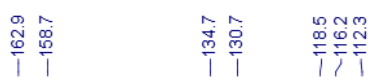

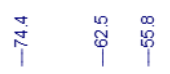

$\stackrel{\oplus}{i}$<smiles>COc1ccc(Br)c(C2=N[C@@H](C)CO2)c1</smiles>

LS1 Me

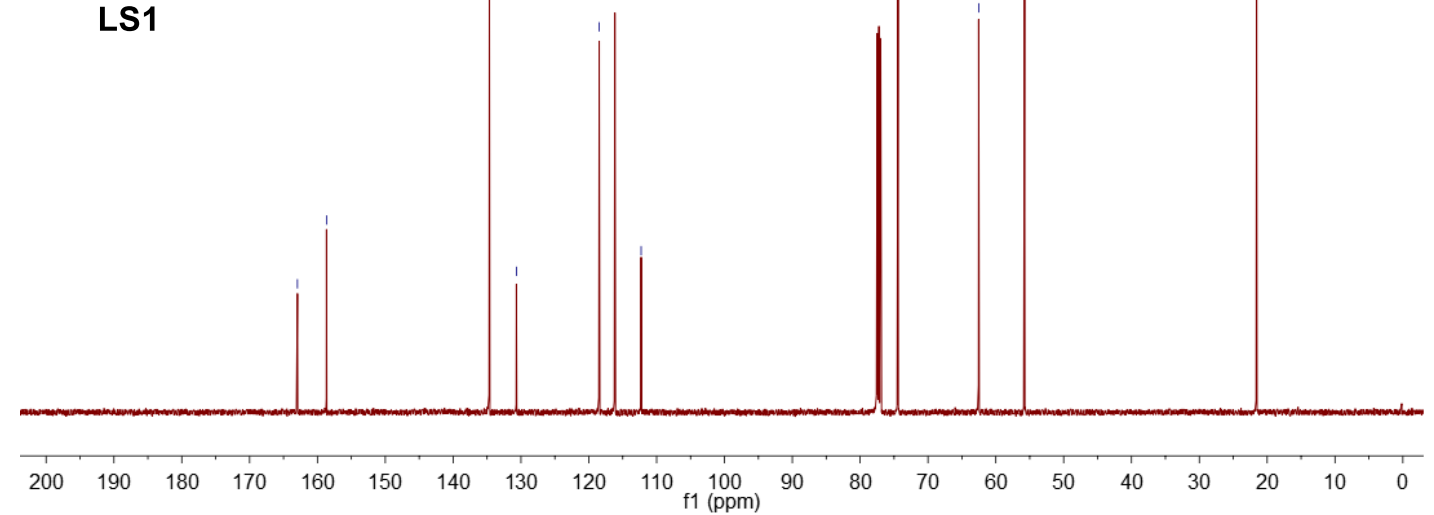


$400 \mathrm{MHz}, \mathrm{CDCl}_{3}$

[L6]

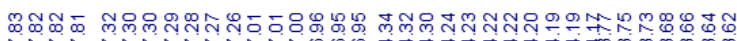

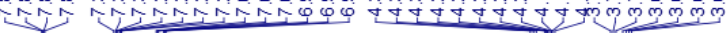
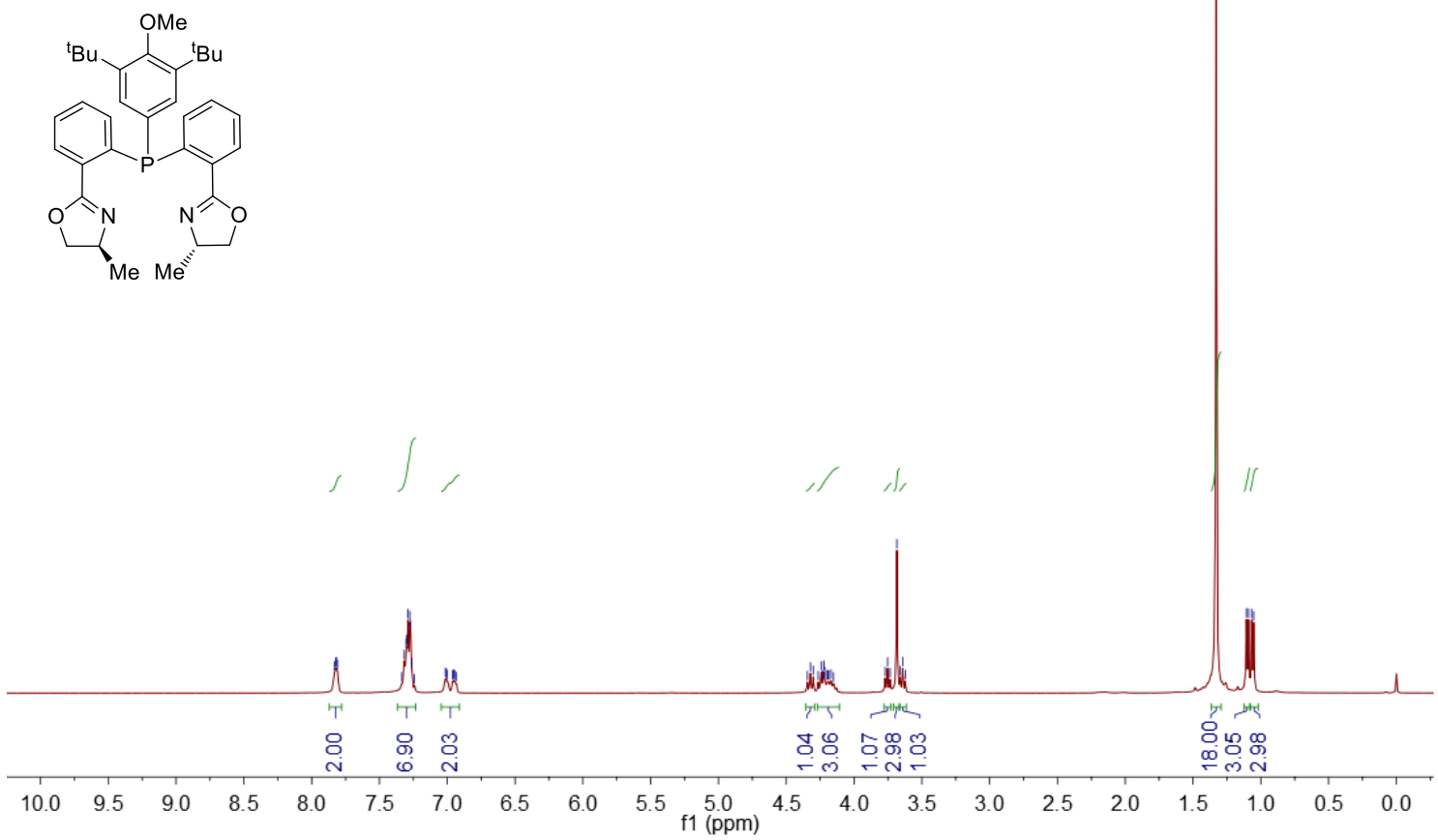

$126 \mathrm{MHz}, \mathrm{CDCl}_{3}$

[L6]

V.

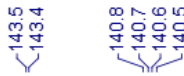

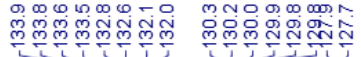

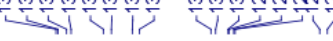
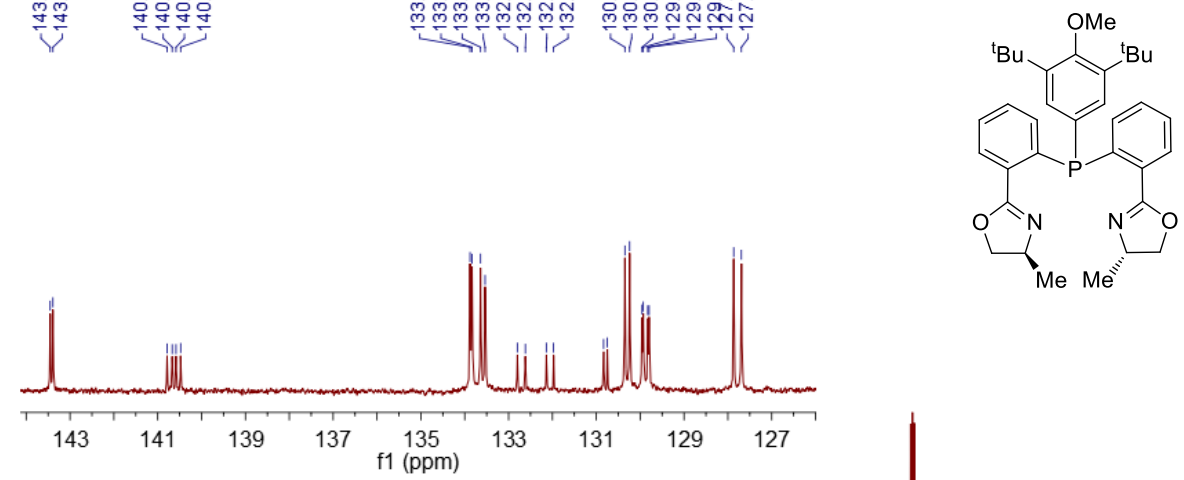

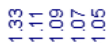

$-7$

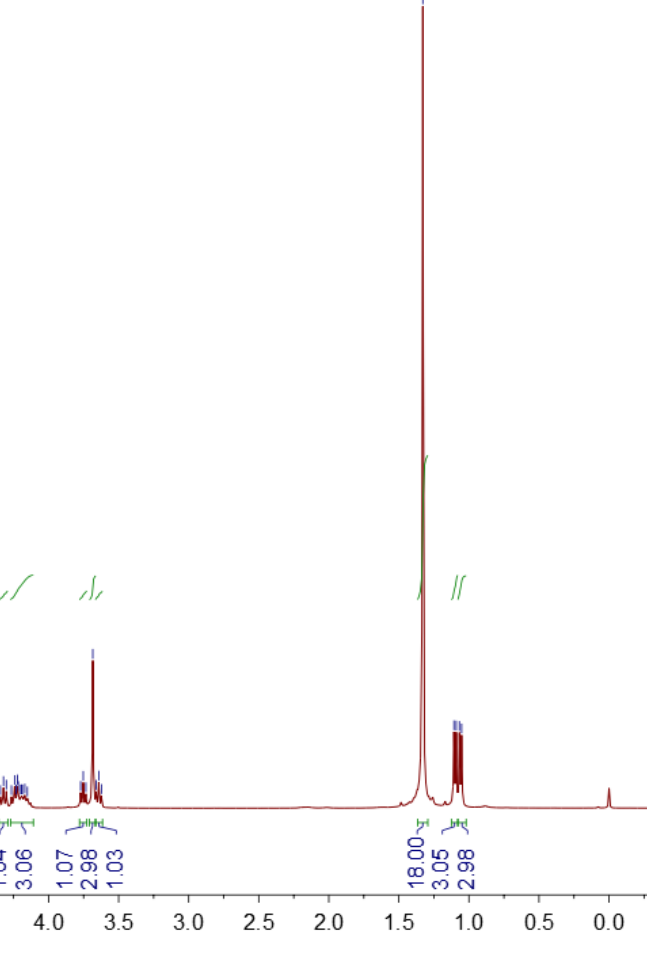


$162 \mathrm{MHz}, \mathrm{CDCl}_{3}$

[L6]

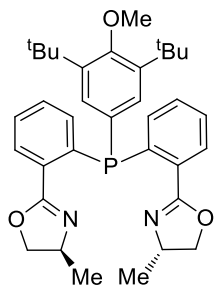

$\begin{array}{lllllllllllllllllllllllllllllllll}6 & 5 & 4 & 3 & 2 & 1 & 0 & -1 & -2 & -3 & -4 & -5 & -6 & -7 & -8 & -9 & -10 & -11 & -12 & -13 & -14 & -15 & -16 & -17 & -18 & -19\end{array}$ $500 \mathrm{MHz}, \mathrm{CDCl}_{3}$

[L7]<smiles>COc1ccc(P(c2ccc(Br)c(C3=N[C@@H](C)CO3)c2)c2ccc(OC)cc2C(=O)c2ccccc2)cc1</smiles>

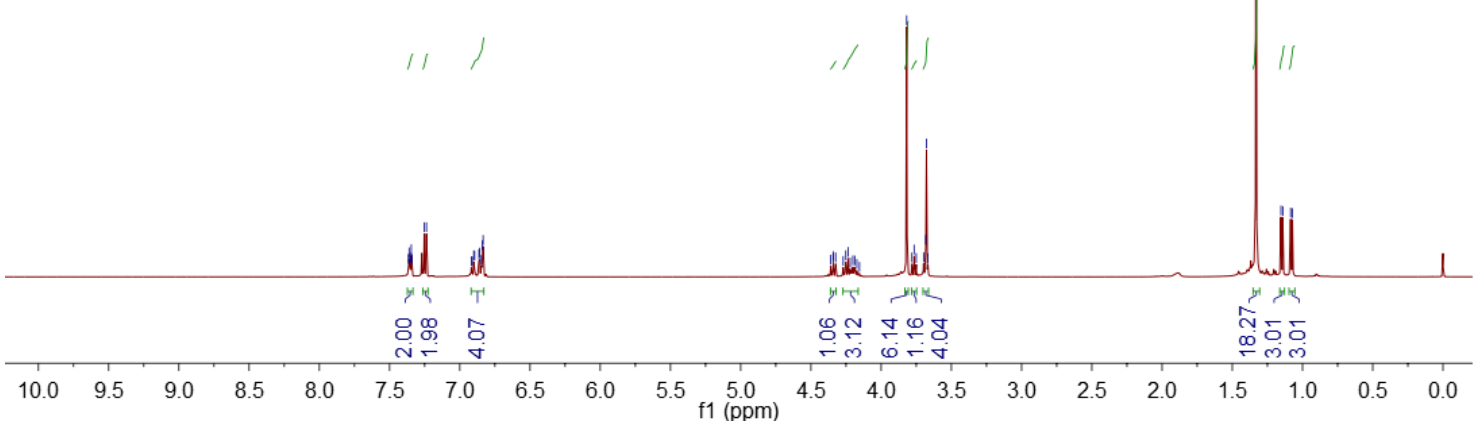


$126 \mathrm{MHz}, \mathrm{CDCl}_{3}$

[L7]

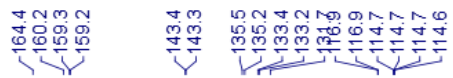

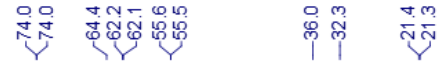

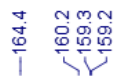

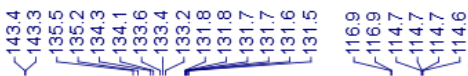
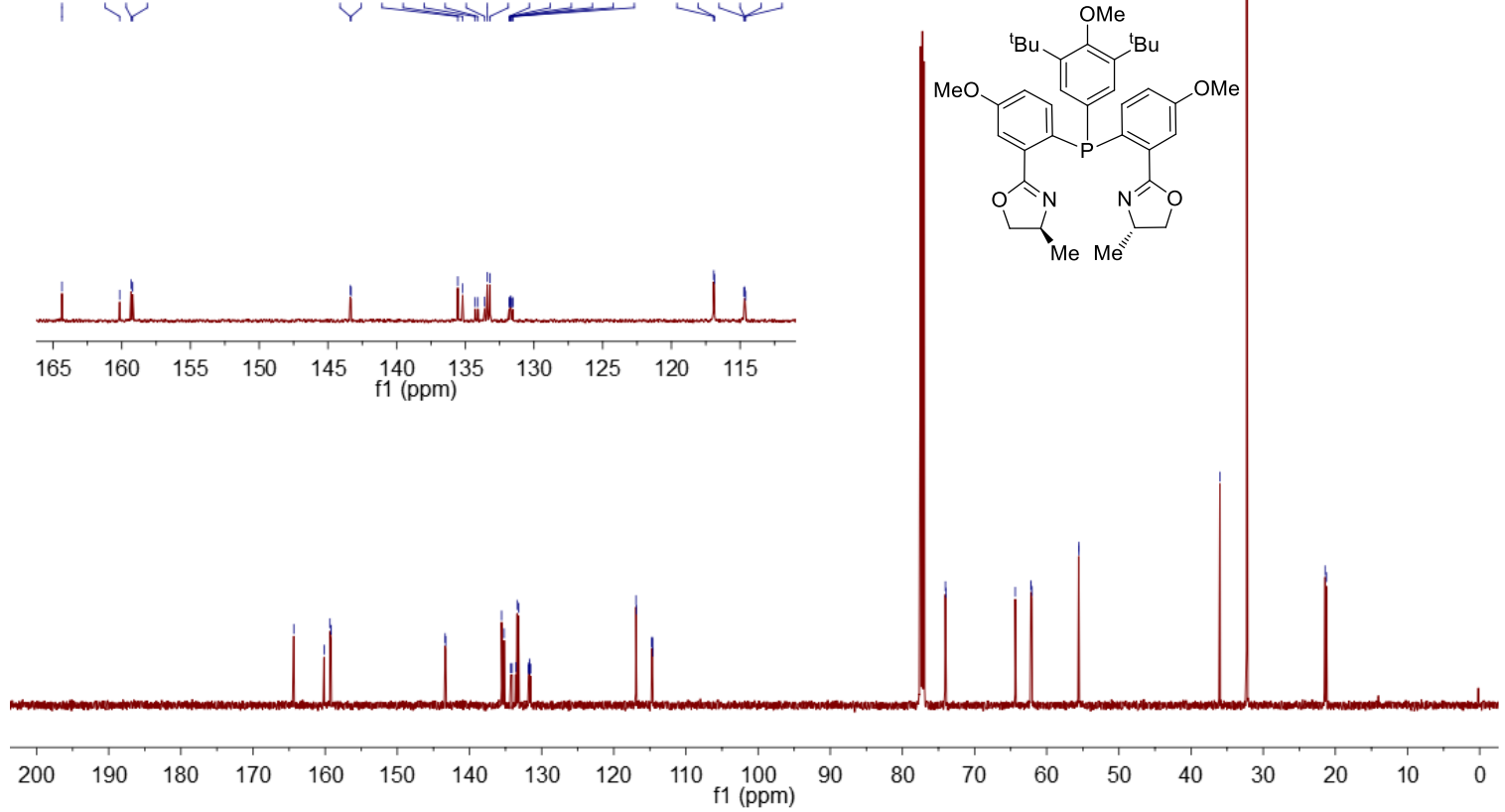

$202 \mathrm{MHz}^{\mathrm{CDCl}_{3}}$

[L7]
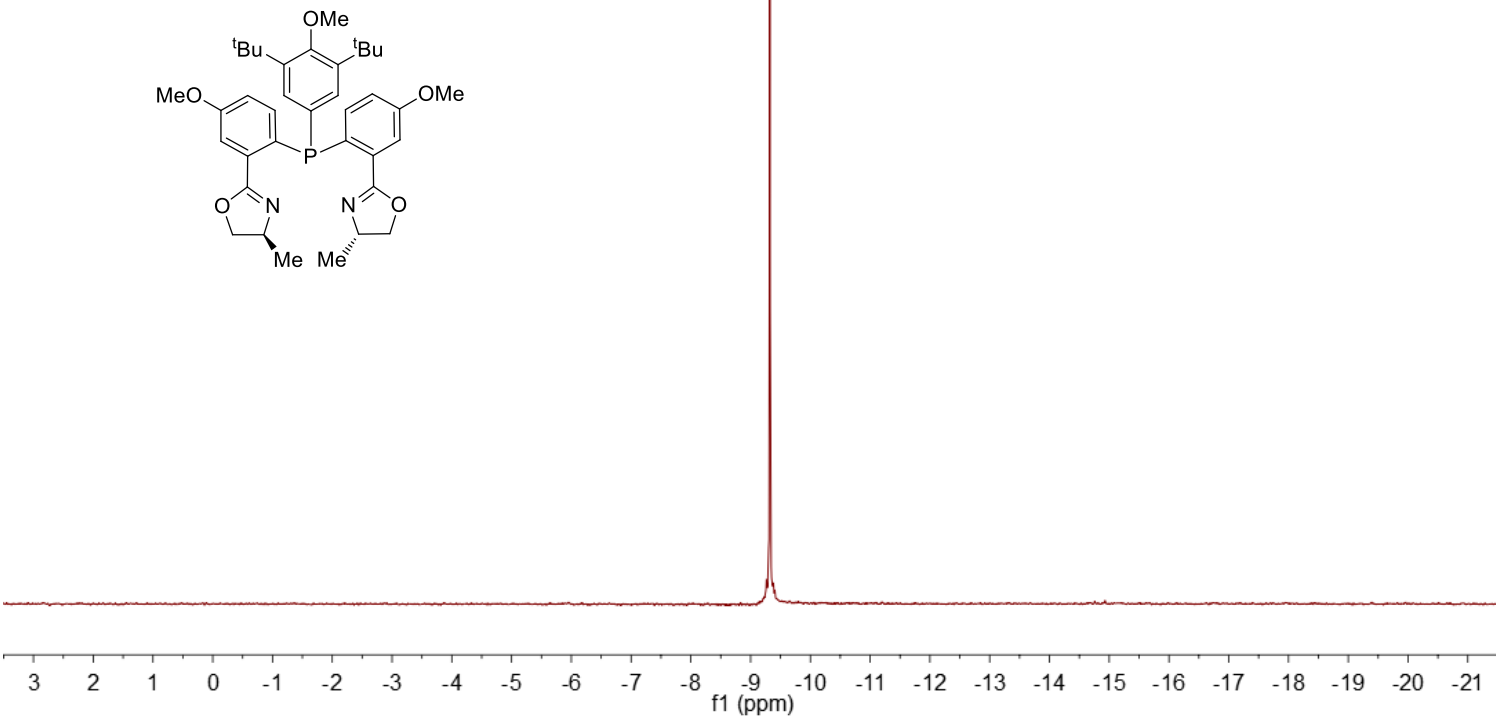
$400 \mathrm{MHz}, \mathrm{CDCl}_{3}$

[L8]<smiles>C[C@H]1COC(c2ccccc2P(C)c2ccccc2C2=N[C@@H](C)CO2)=N1</smiles>

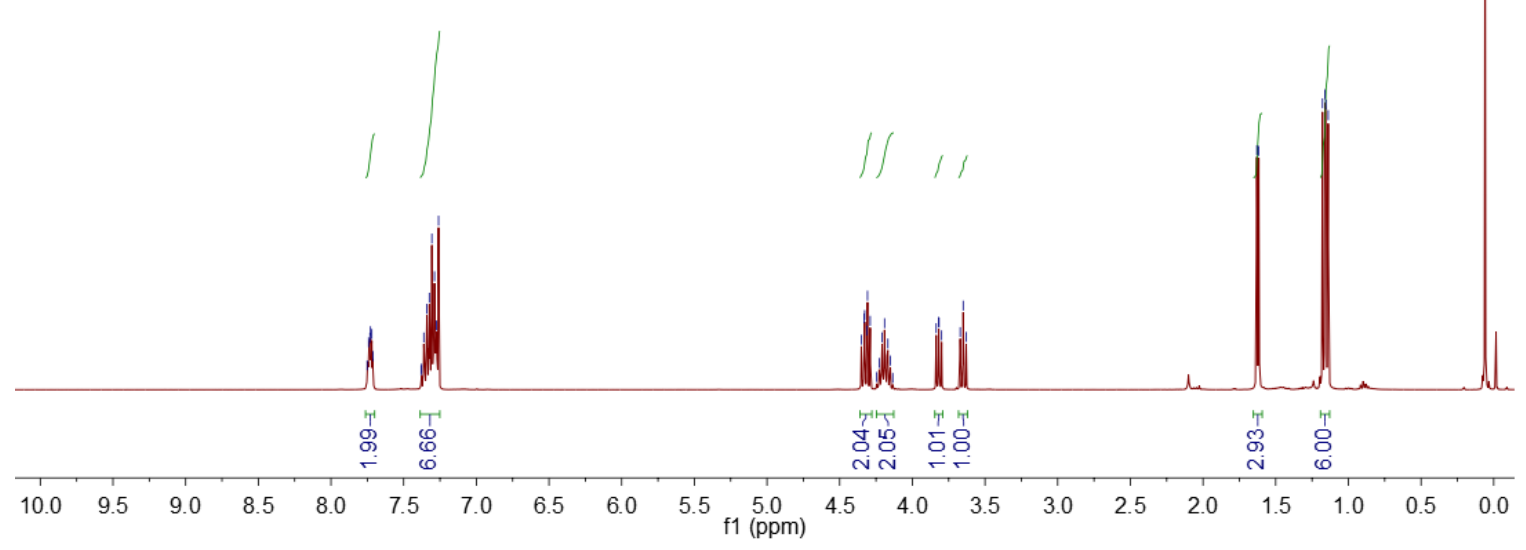

$101 \mathrm{MHz}, \mathrm{CDCl}_{3}$

[L8]

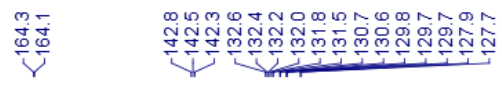
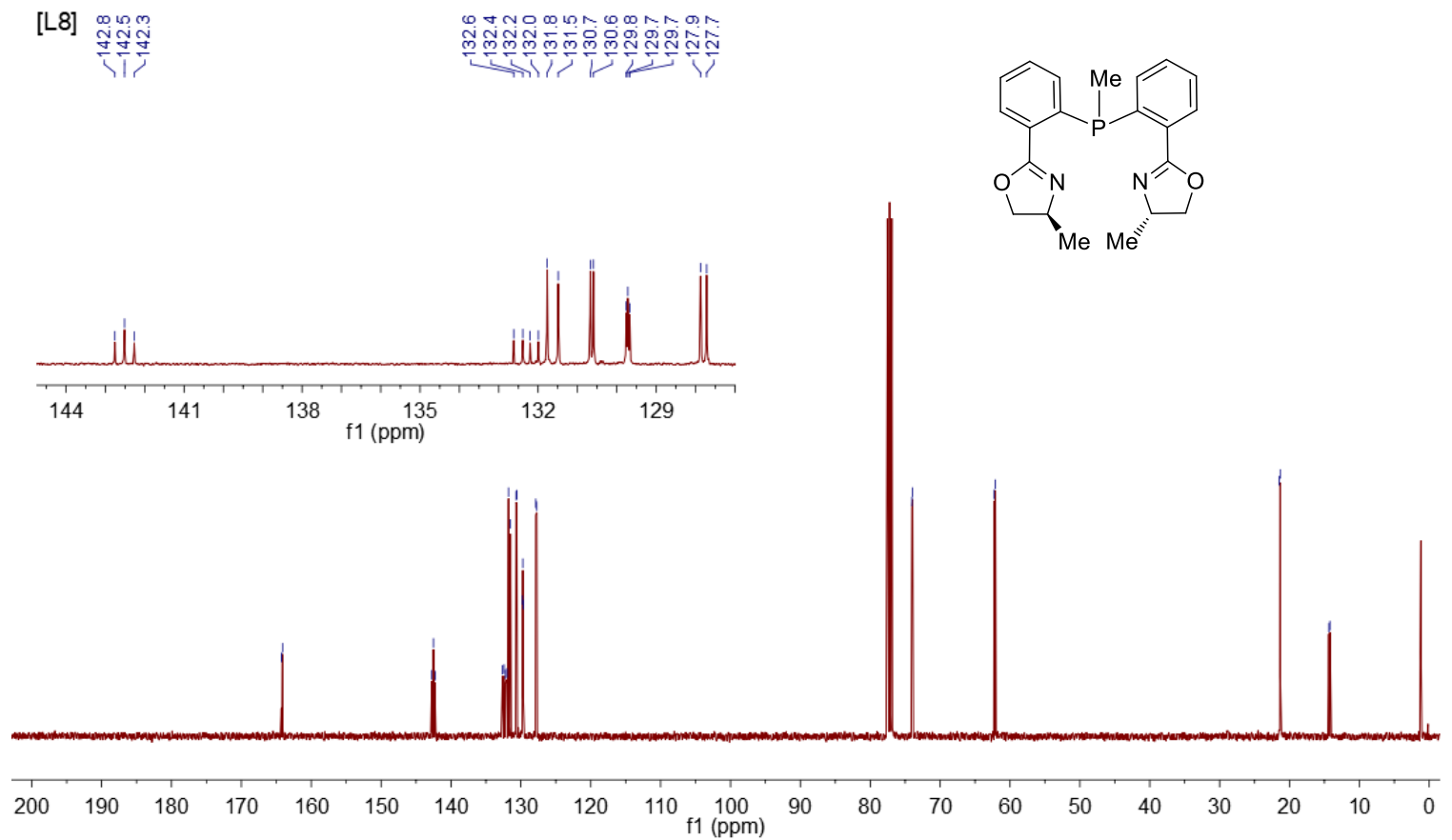
$162 \mathrm{MHz} \mathrm{CDCl}_{3}$

[L8]

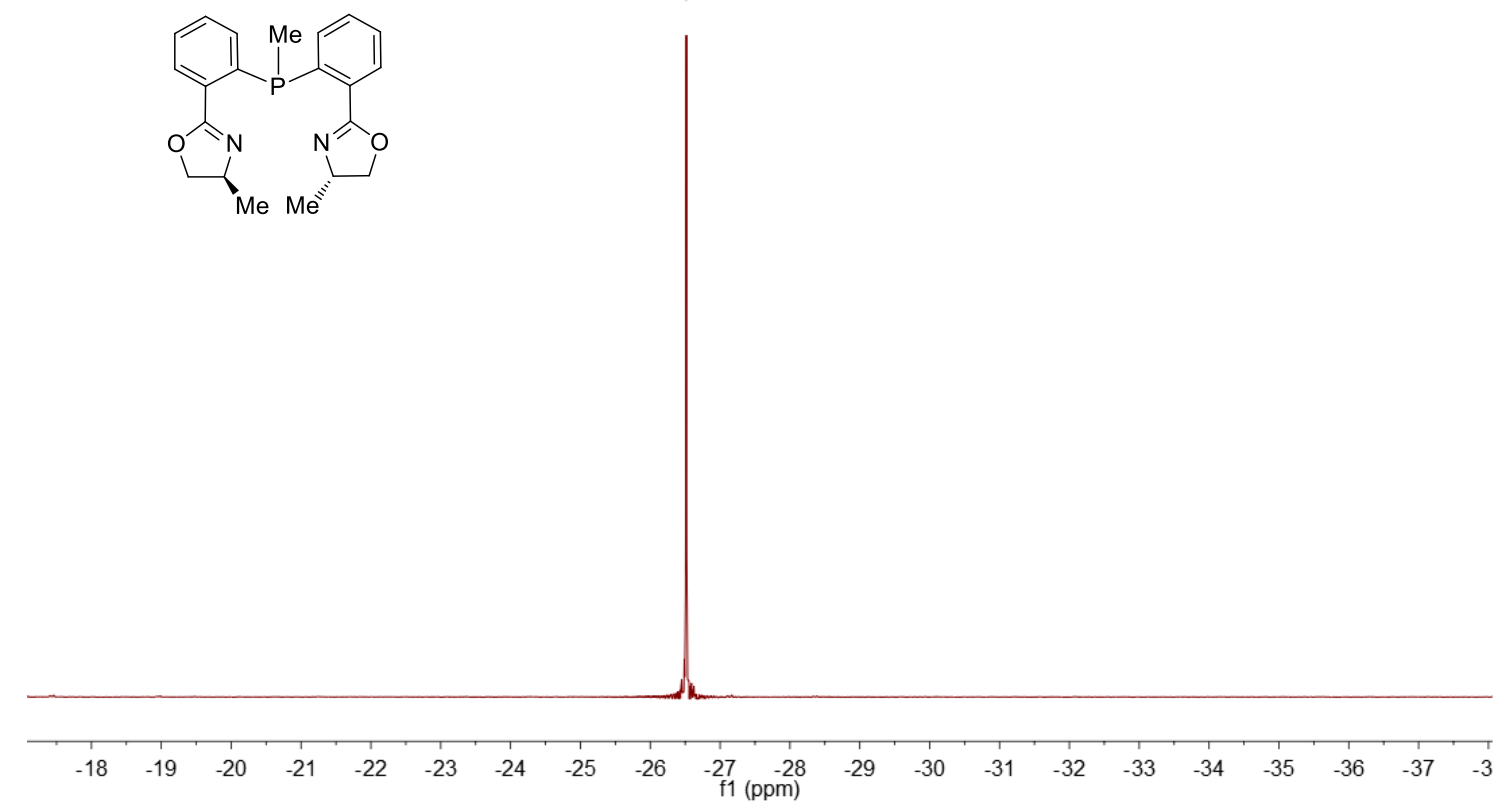

$400 \mathrm{MHz}, \mathrm{CDCl}_{3}$

[L9]

$\mathrm{MeO}$

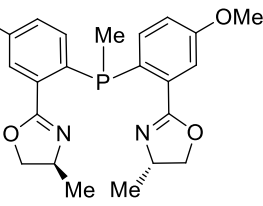

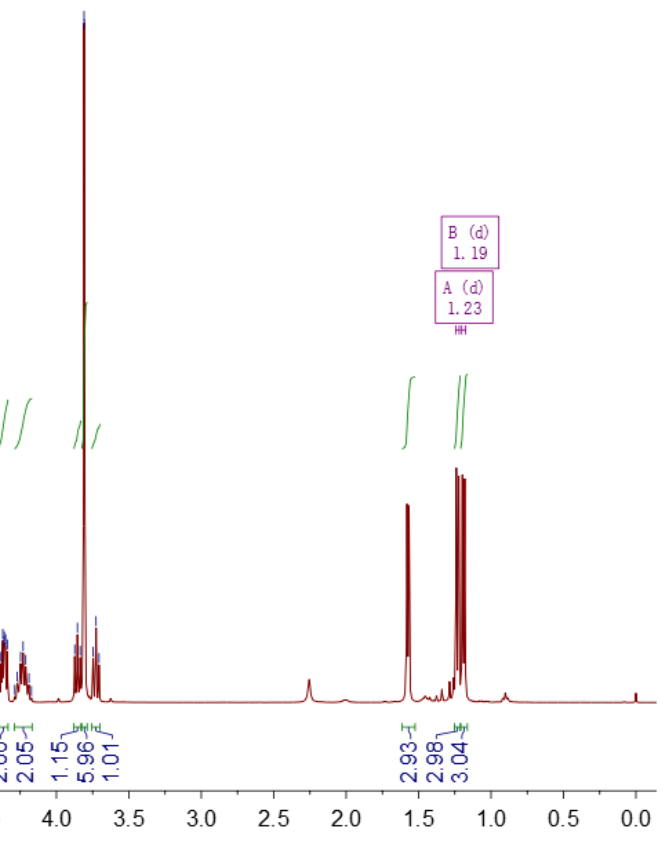


$101 \mathrm{MHz}, \mathrm{CDCl}_{3}$

[L9]
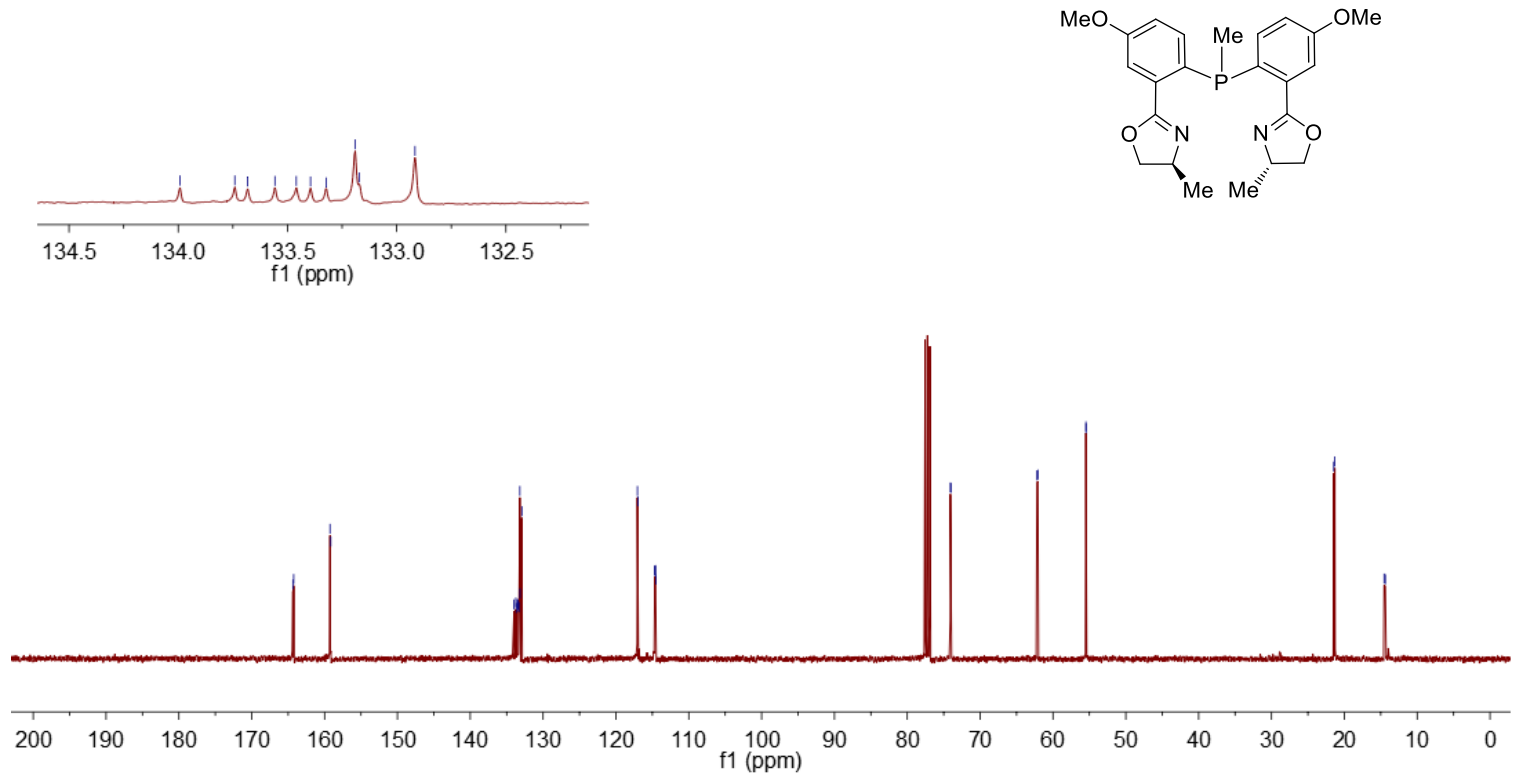

$202 \mathrm{MHz}, \mathrm{CDCl}_{3}$

[L9]

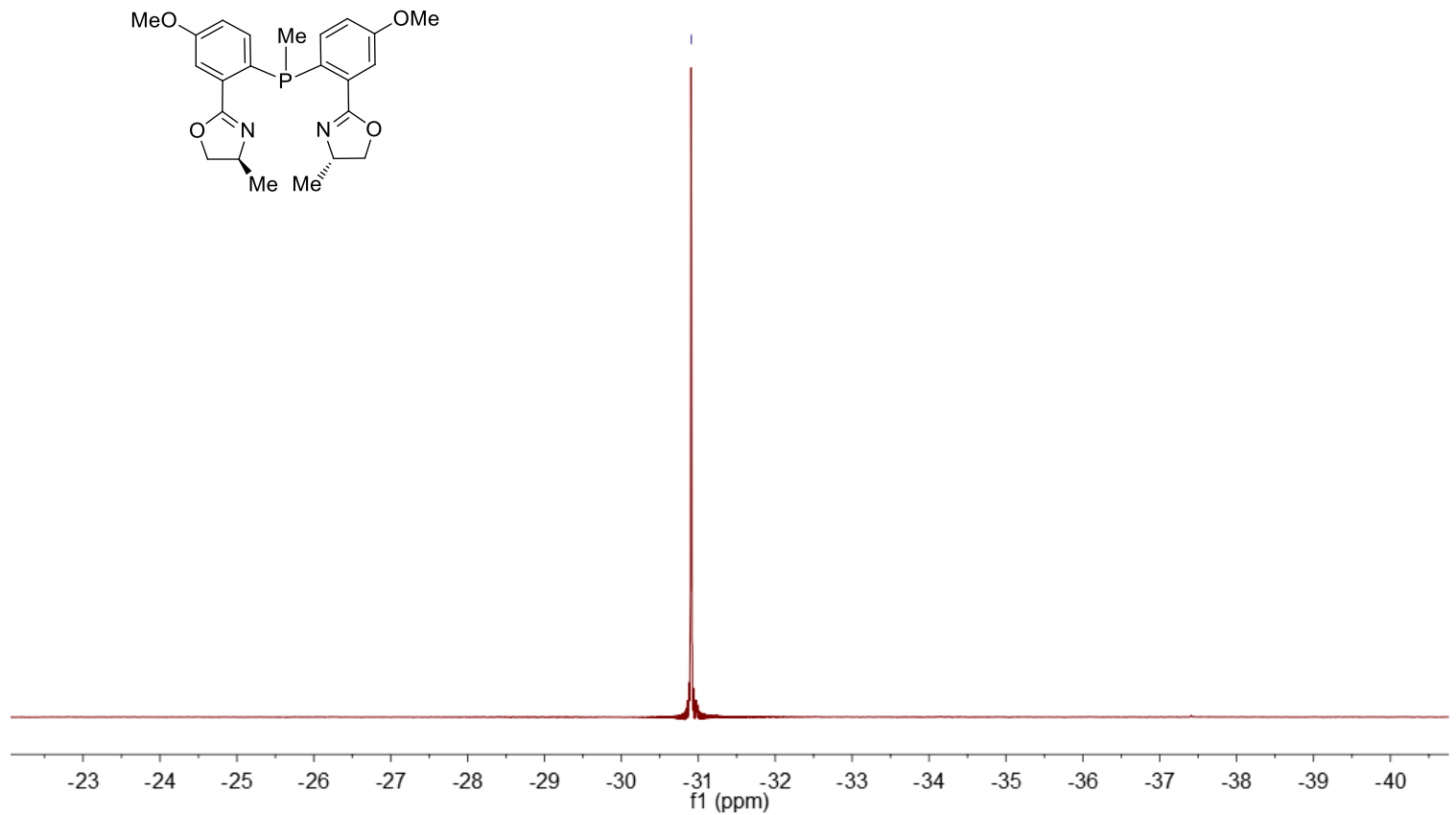


$400 \mathrm{MHz}, \mathrm{CDCl}_{3}$

[3aa]<smiles>C=C[C@@H](CCc1ccccc1)C(C#N)C#N</smiles>

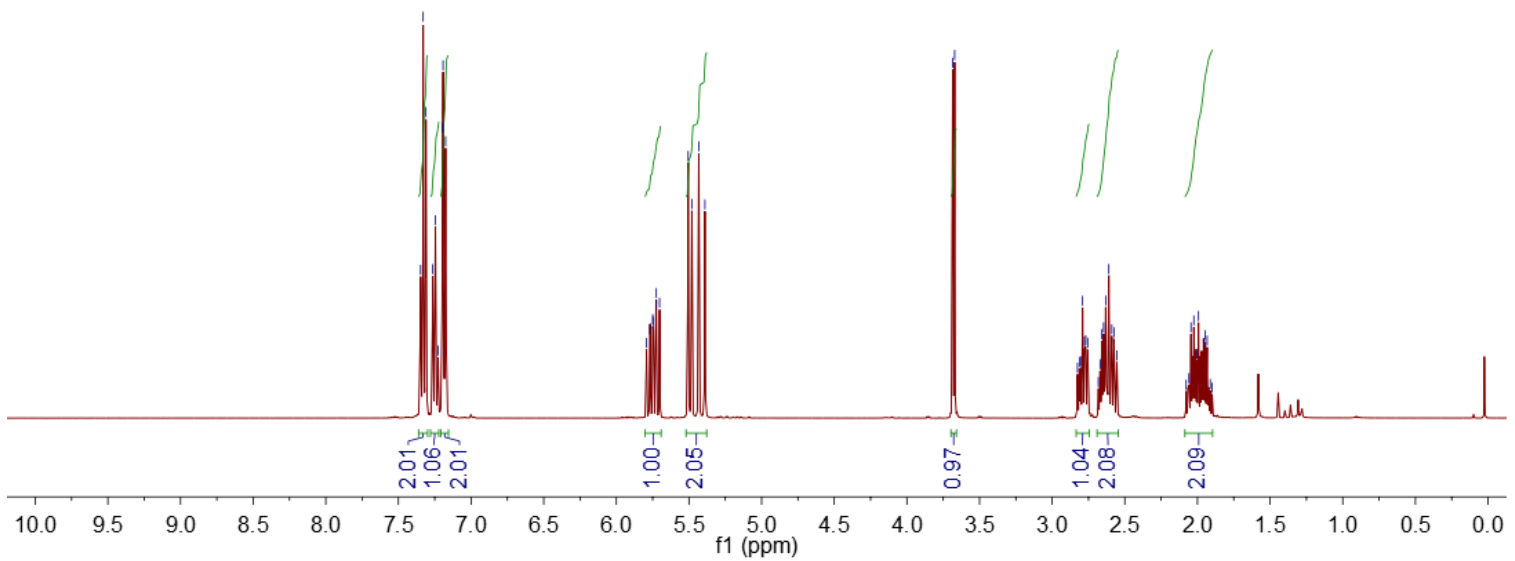

$101 \mathrm{MHz}, \mathrm{CDCl}_{3}$

[3aa]

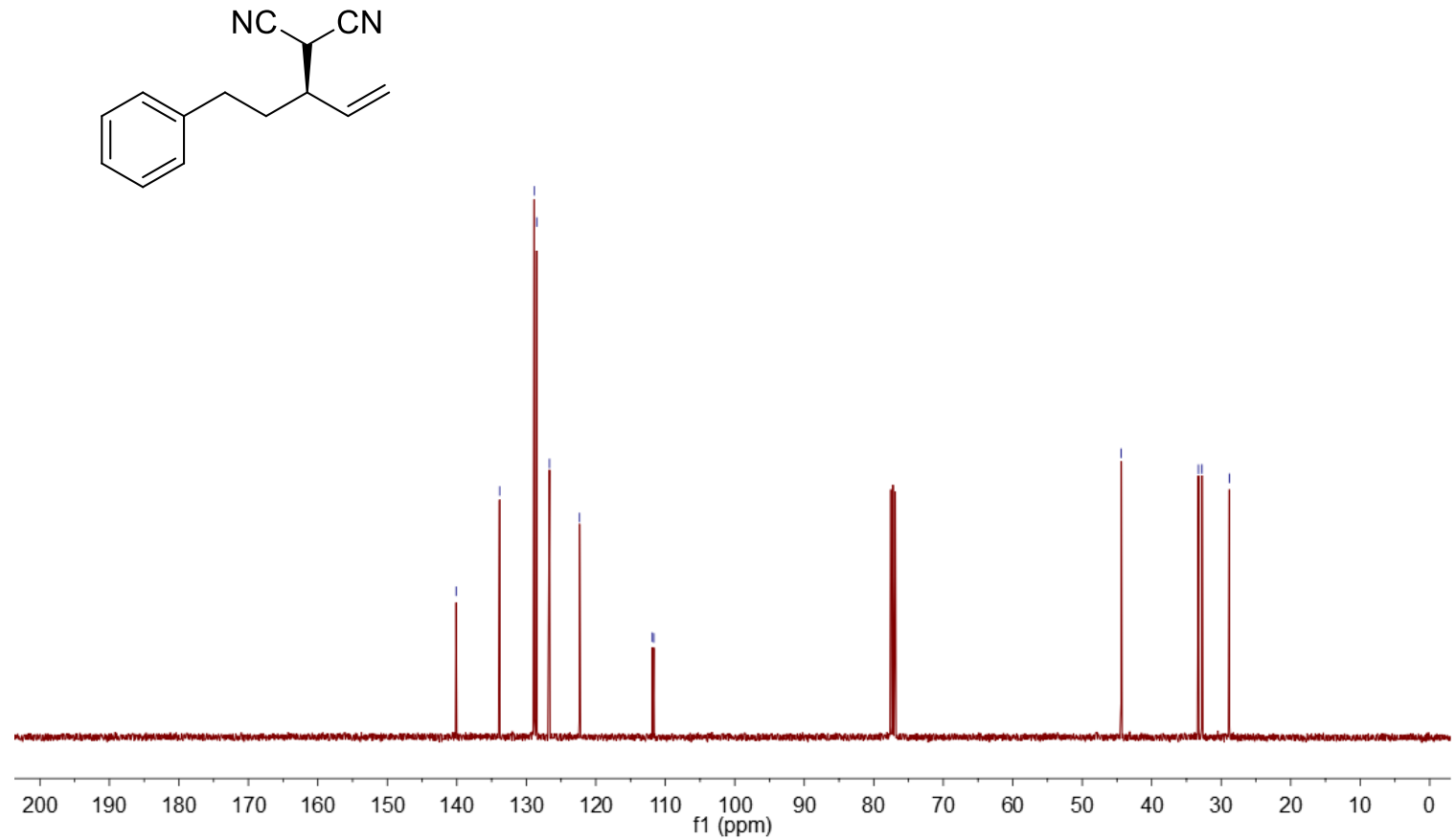


$400 \mathrm{MHz}, \mathrm{CDCl}_{3}$

[3ab]
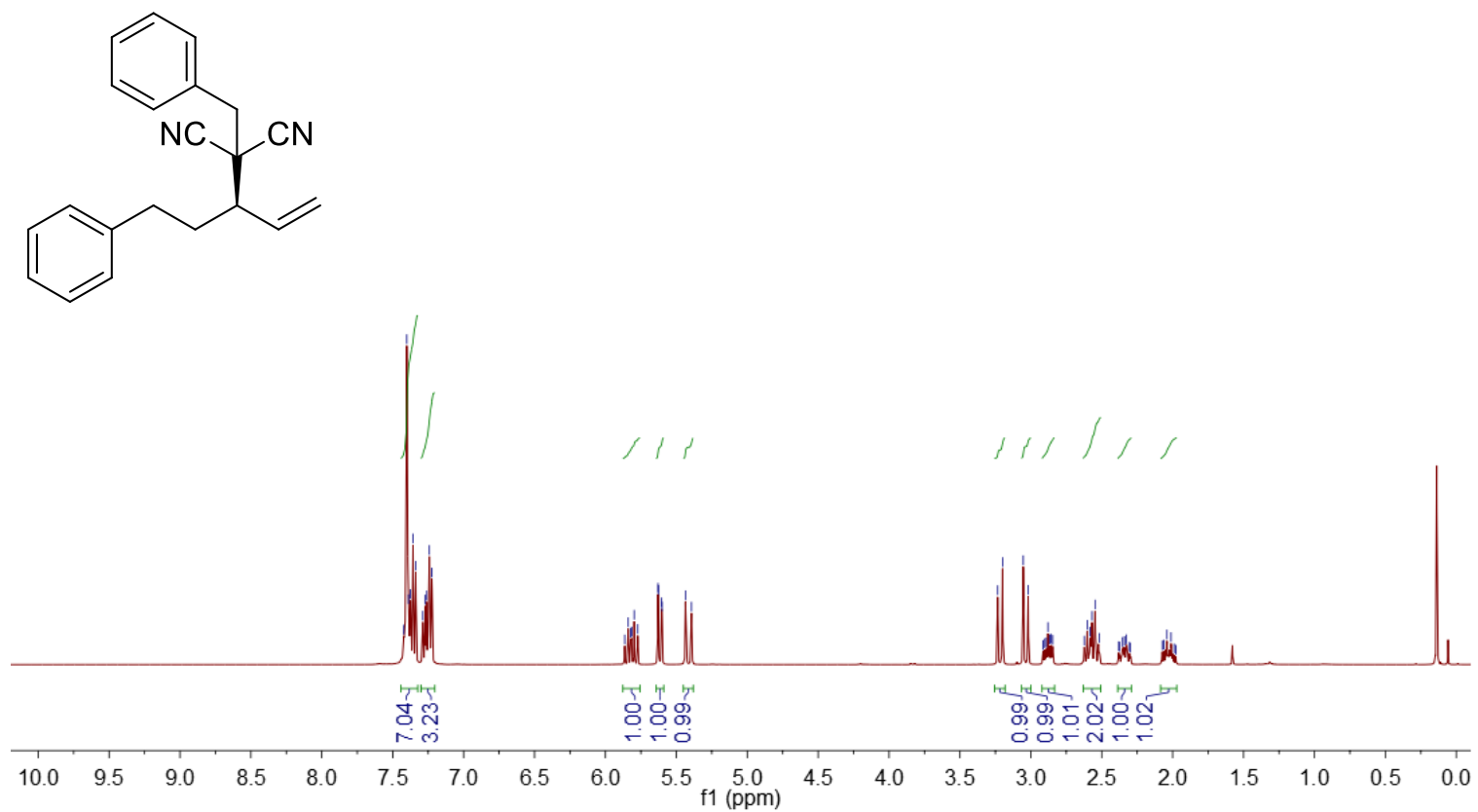

$126 \mathrm{MHz}, \mathrm{CDCl}_{3}$

[3ab]

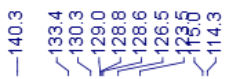

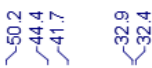

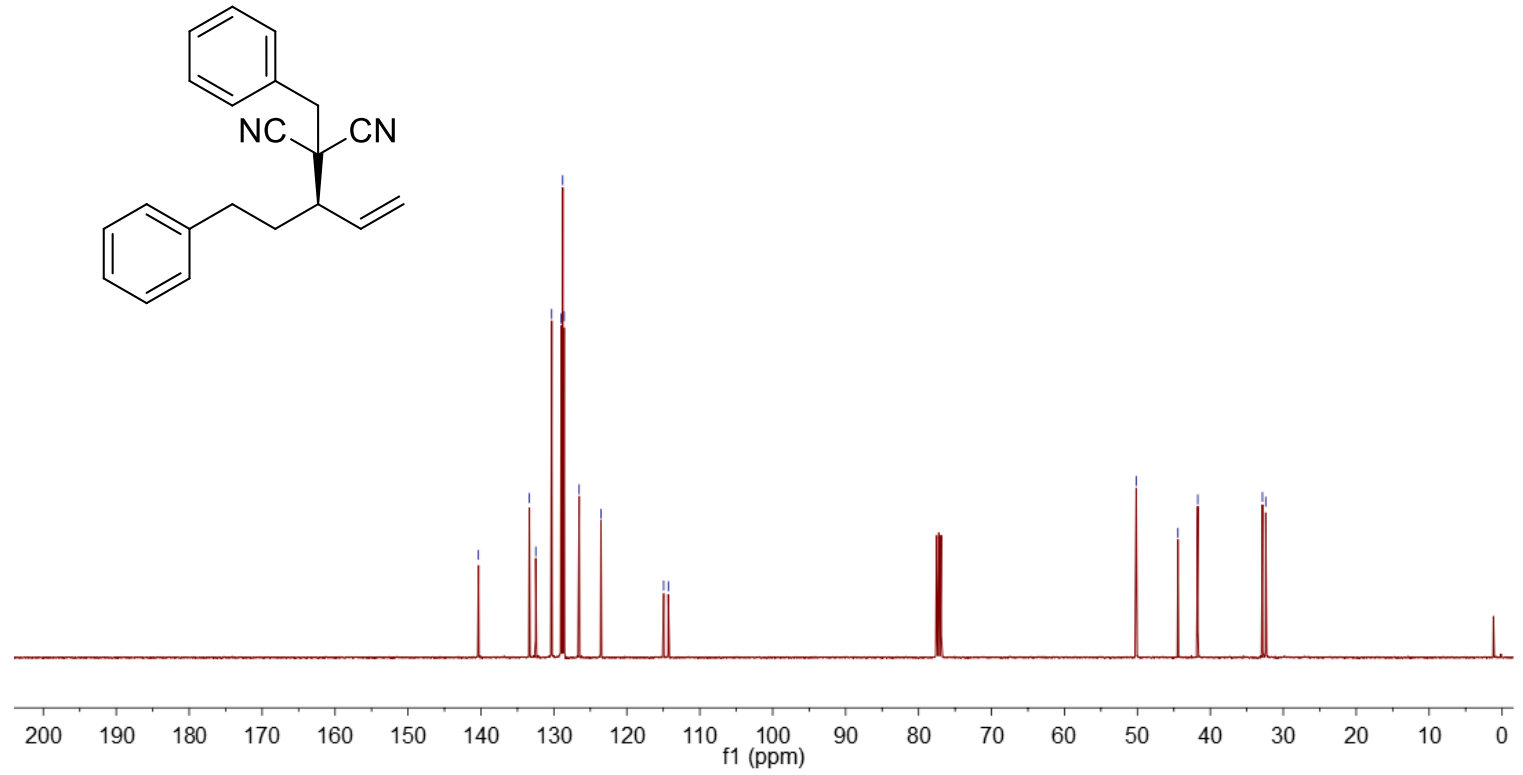


$400 \mathrm{MHz}, \mathrm{CDCl}_{3}$

[3ac]<smiles>C=C[C@@H](CCc1ccccc1)C(C#N)(C#N)Cc1ccc(C#N)cc1</smiles>

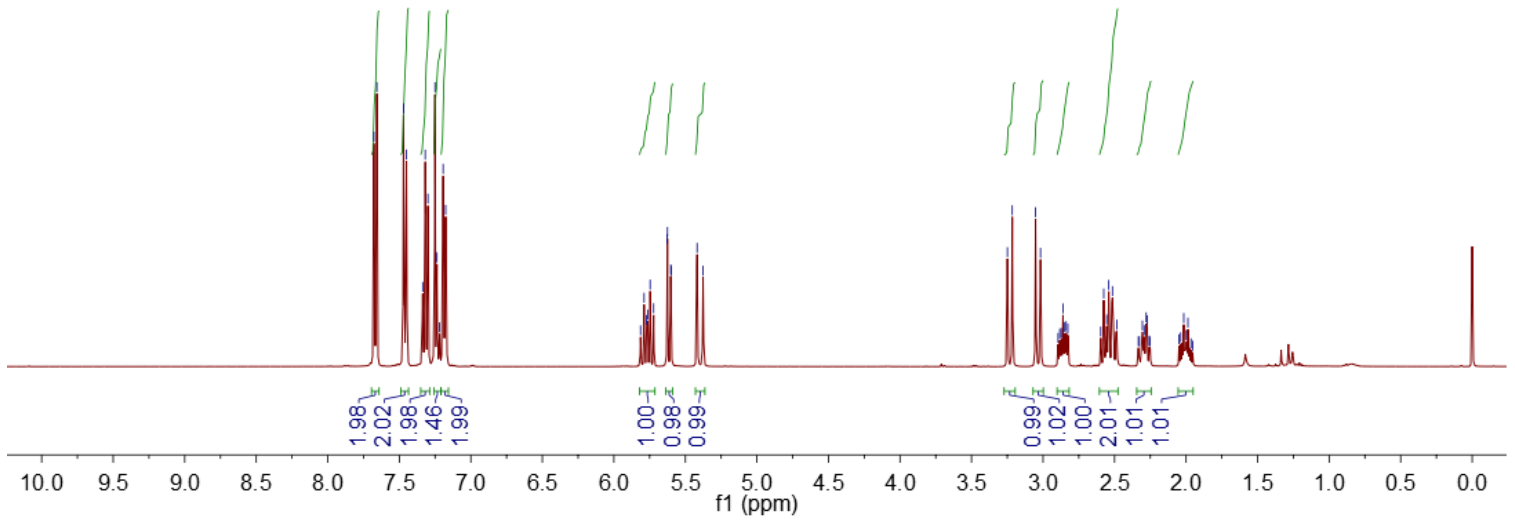

$126 \mathrm{MHz}, \mathrm{CDCl}_{3}$

[3ac]

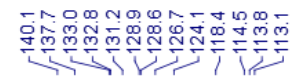

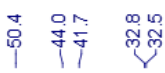

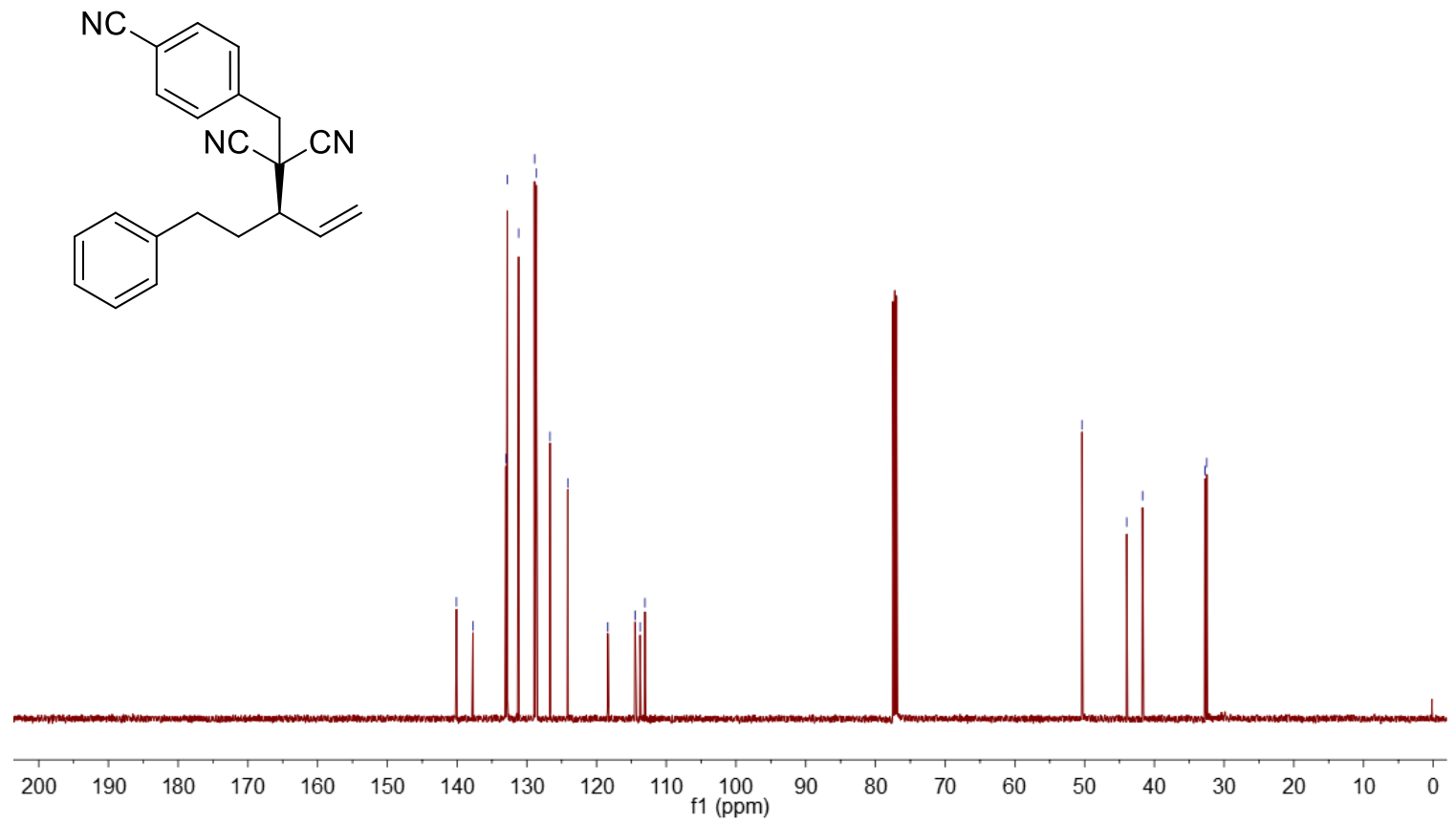


$400 \mathrm{MHz}, \mathrm{CDCl}_{3}$

[3ad]<smiles>C=C[C@@H](CCc1ccccc1)C(C#N)(C#N)Cc1ccc(C(F)(F)F)cc1</smiles>

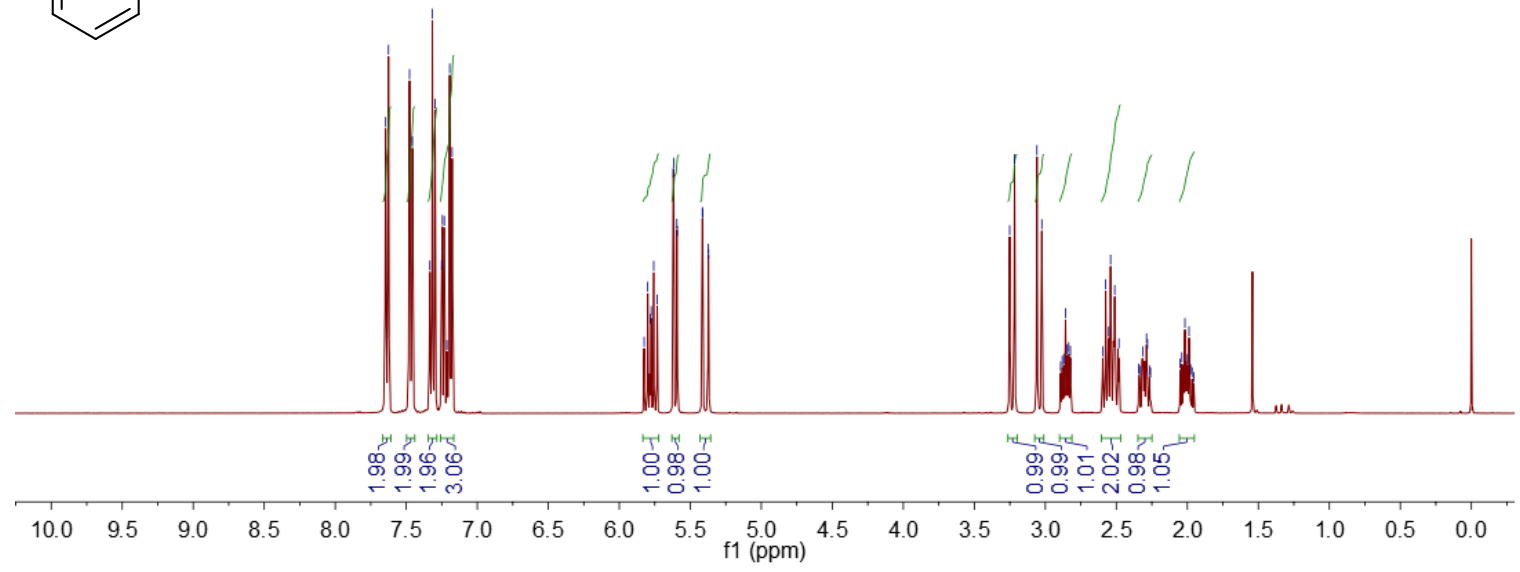

$126 \mathrm{MHz}, \mathrm{CDCl}_{3}$

[3ad]

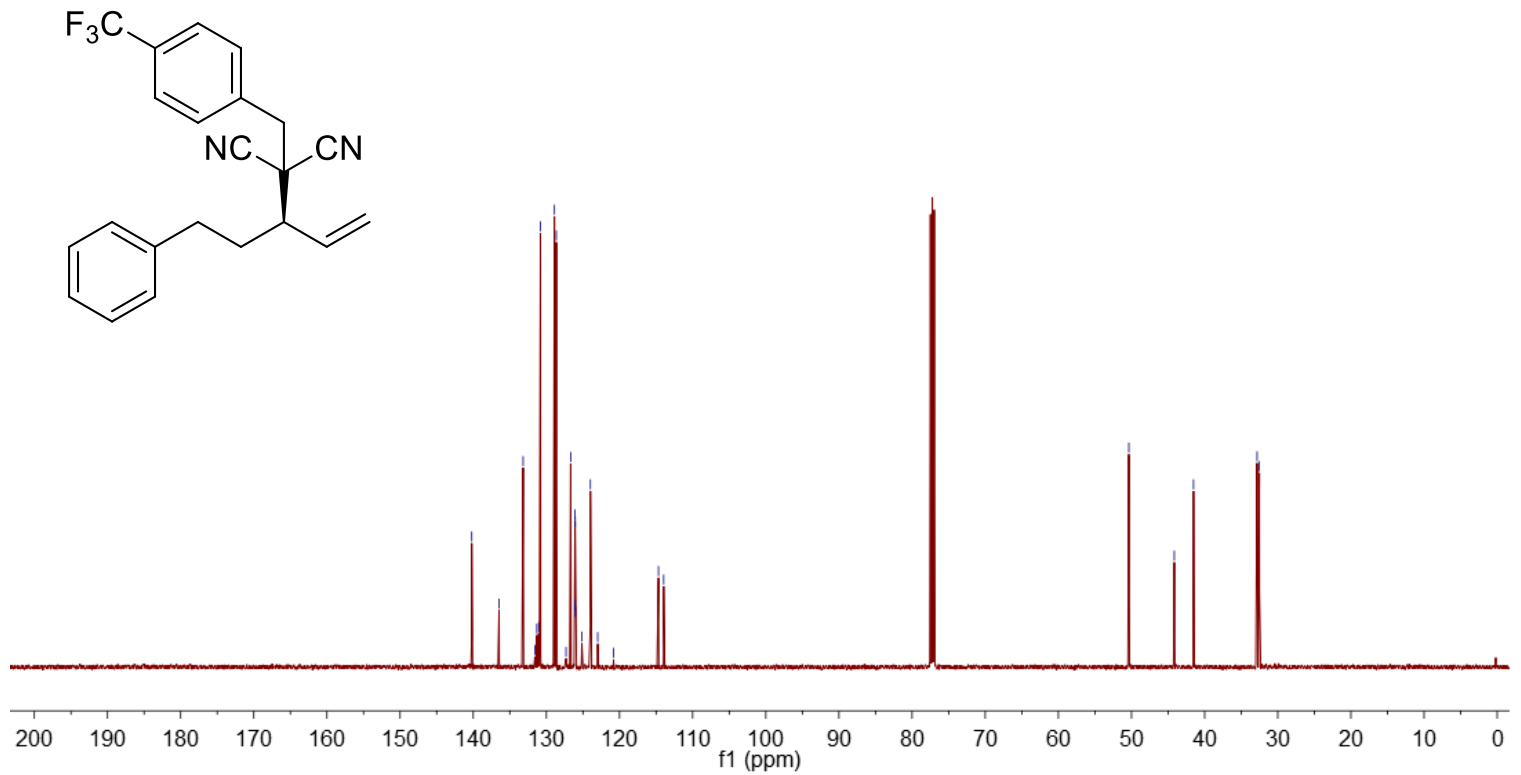


$500 \mathrm{MHz}, \mathrm{CDCl}_{3}$

[3ae]

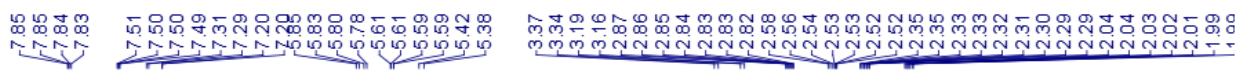<smiles>C=C[C@@H](CCc1ccccc1)C(C#N)(C#N)Cc1ccc2ccccc2c1</smiles>
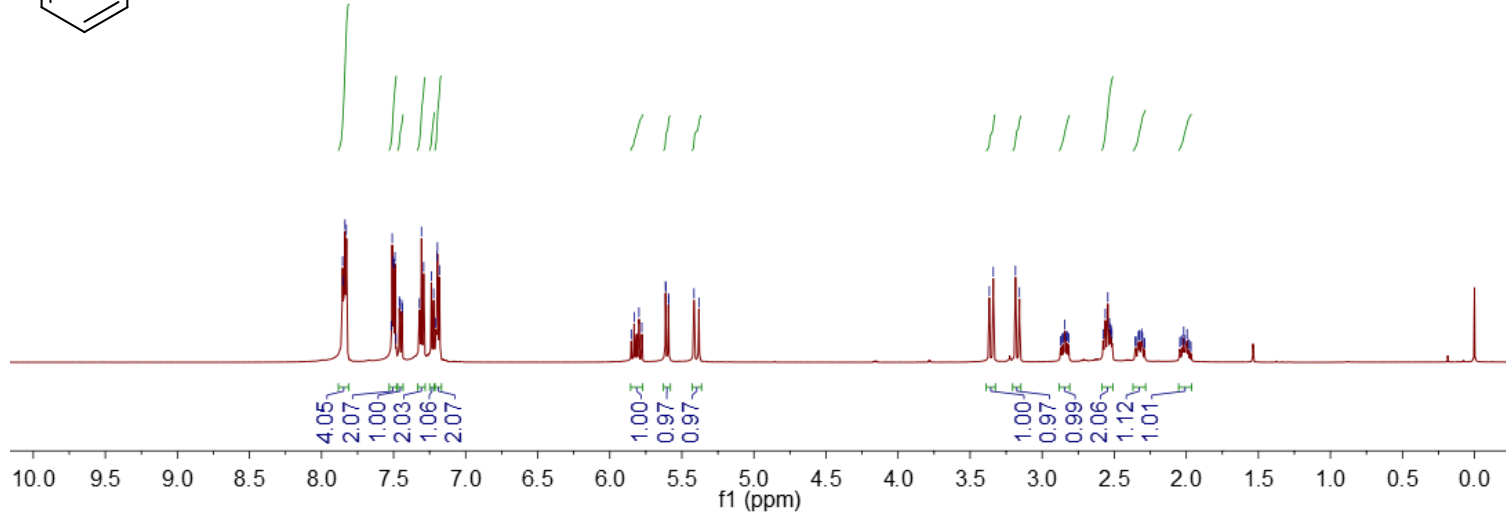

$126 \mathrm{MHz}, \mathrm{CDCl}_{3}$

[3ae]

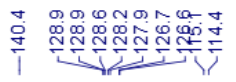

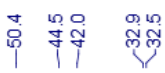<smiles>C=C[C@@H](CCc1ccccc1)C(C#N)(C#N)Cc1ccc2ccccc2c1</smiles>

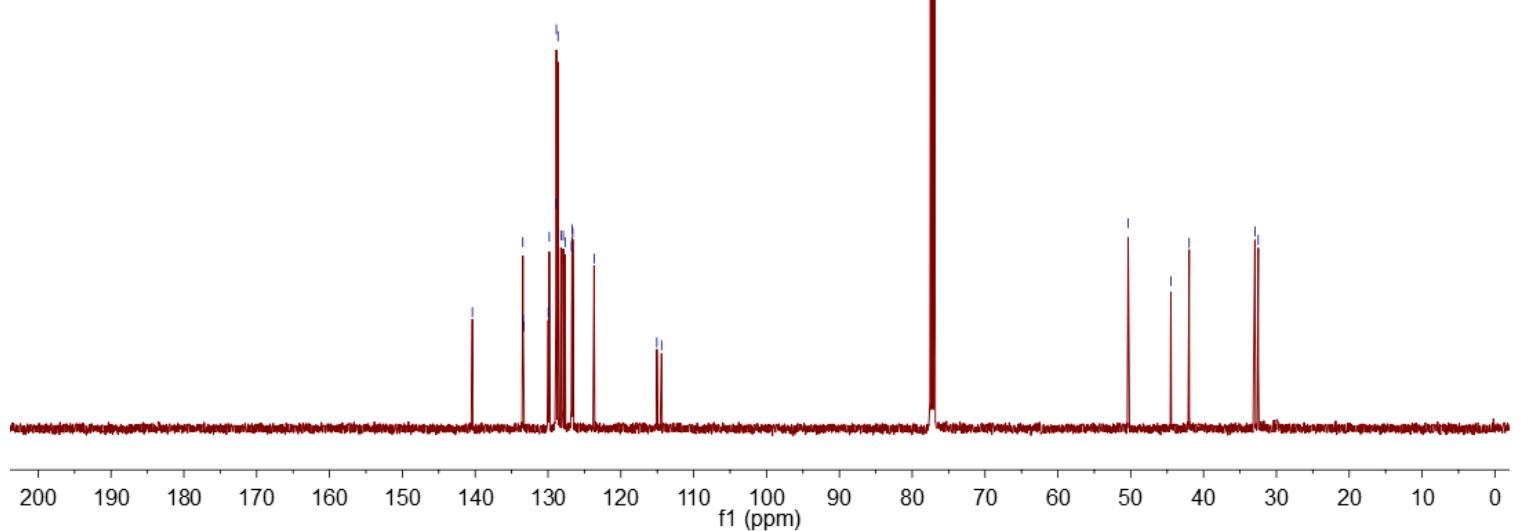


$400 \mathrm{MHz}, \mathrm{CDCl}_{3}$

[3af]

m<smiles>C=C[C@@H](CCc1ccccc1)C(C#N)(C#N)Cc1cccn1C</smiles>

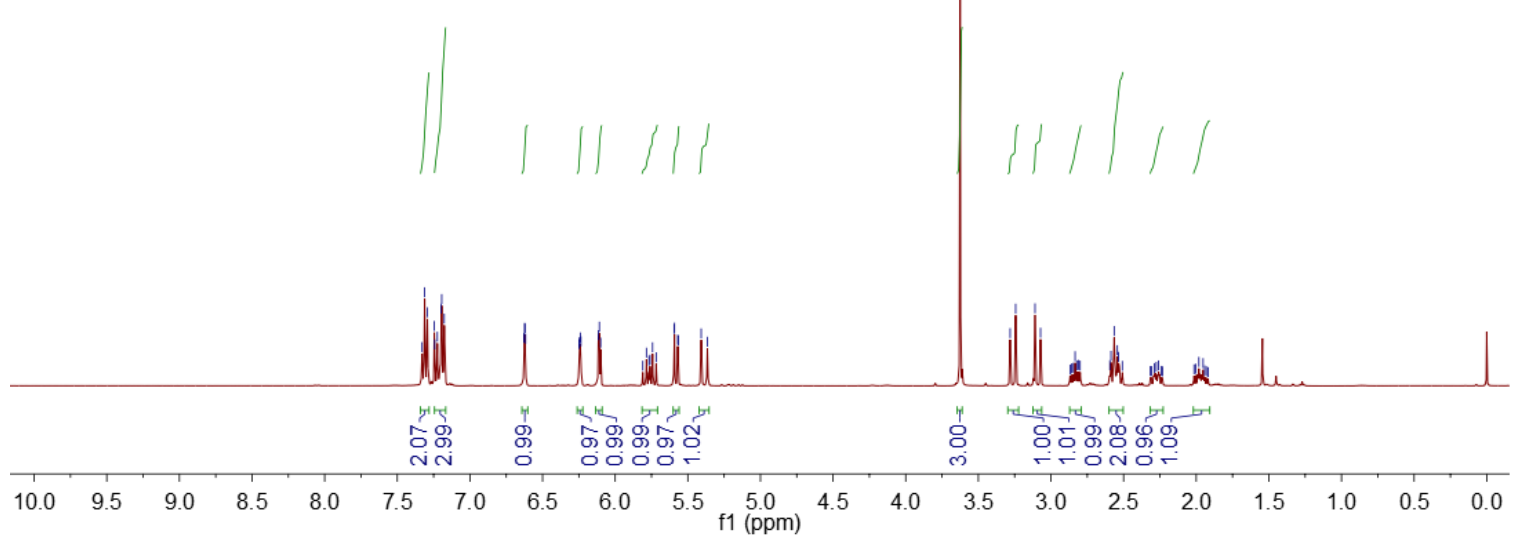

$126 \mathrm{MHz}, \mathrm{CDCl}_{3}$

[3af]<smiles>C=C[C@@H](CCc1ccccc1)C(C#N)(C#N)Cc1cccn1C</smiles>

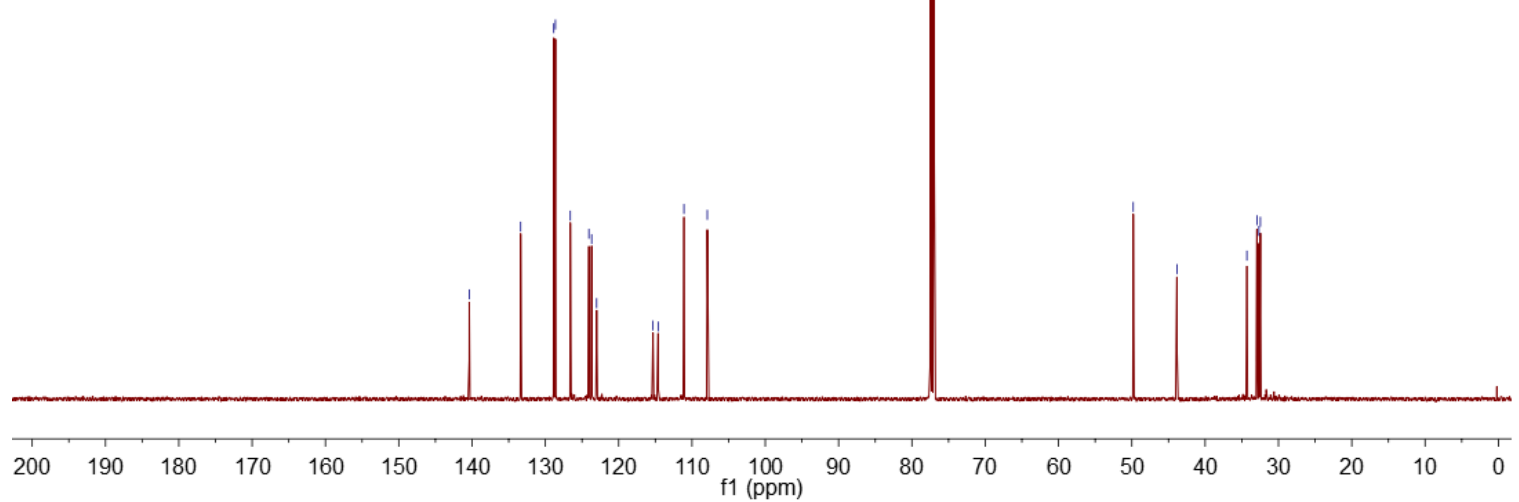


$400 \mathrm{MHz}, \mathrm{CDCl}_{3}$

[3ag]

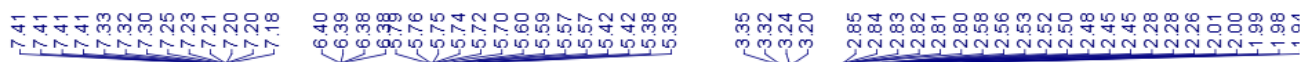<smiles>C=C[C@@H](CCc1ccccc1)C(C#N)(C#N)Cc1ccco1</smiles>
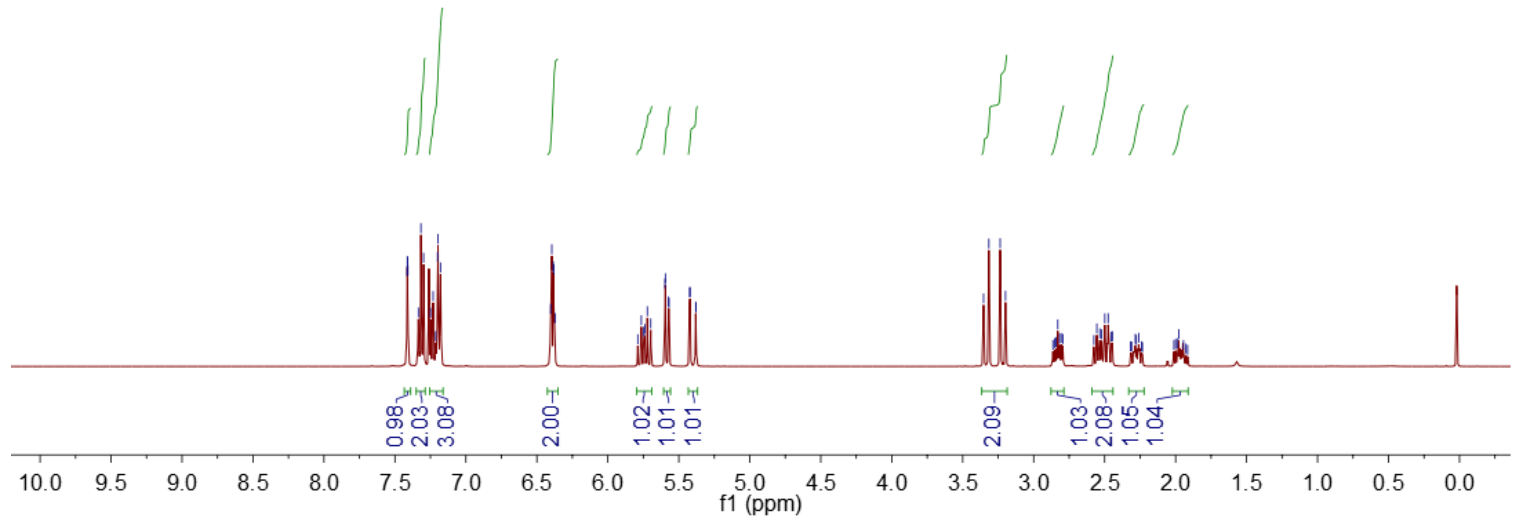

$126 \mathrm{MHz}, \mathrm{CDCl}_{3}$

[3ag] 宁<smiles>C=C[C@@H](CCc1ccccc1)C(C#N)(C#N)Cc1ccco1</smiles>

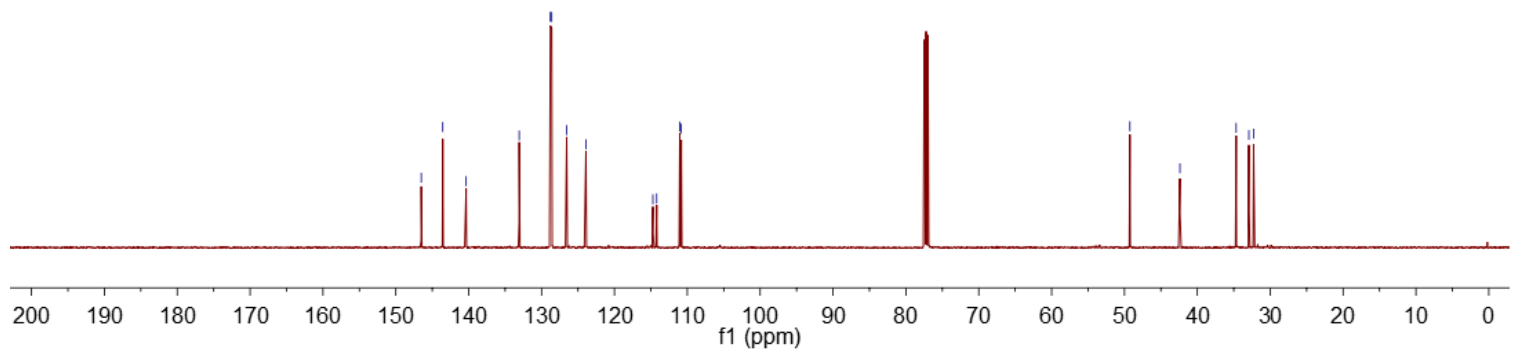


$400 \mathrm{MHz}, \mathrm{CDCl}_{3}$

[3ah]

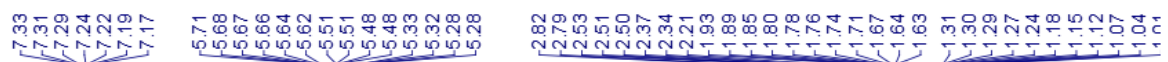<smiles>C=C[C@@H](CCc1ccccc1)C(C#N)(C#N)CC1CCCCC1</smiles>

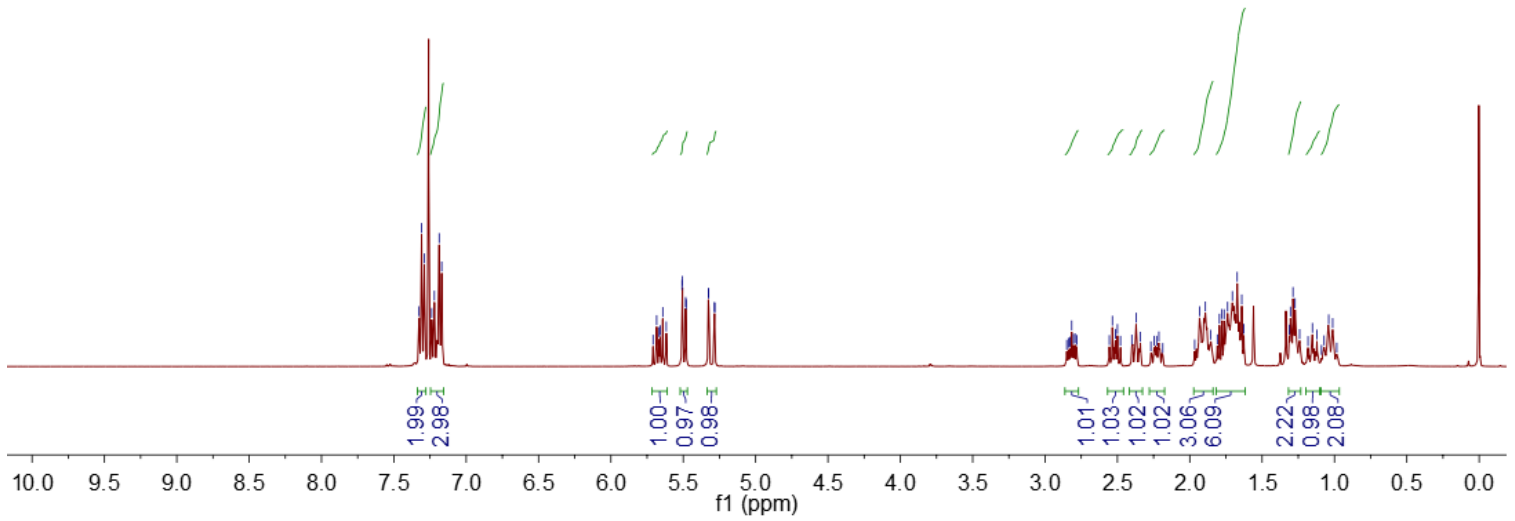
$126 \mathrm{MHz}, \mathrm{CDCl}_{3}$

[3ah]<smiles>C=C[C@@H](CCc1ccccc1)C(C#N)(C#N)CC1CCCCC1</smiles>

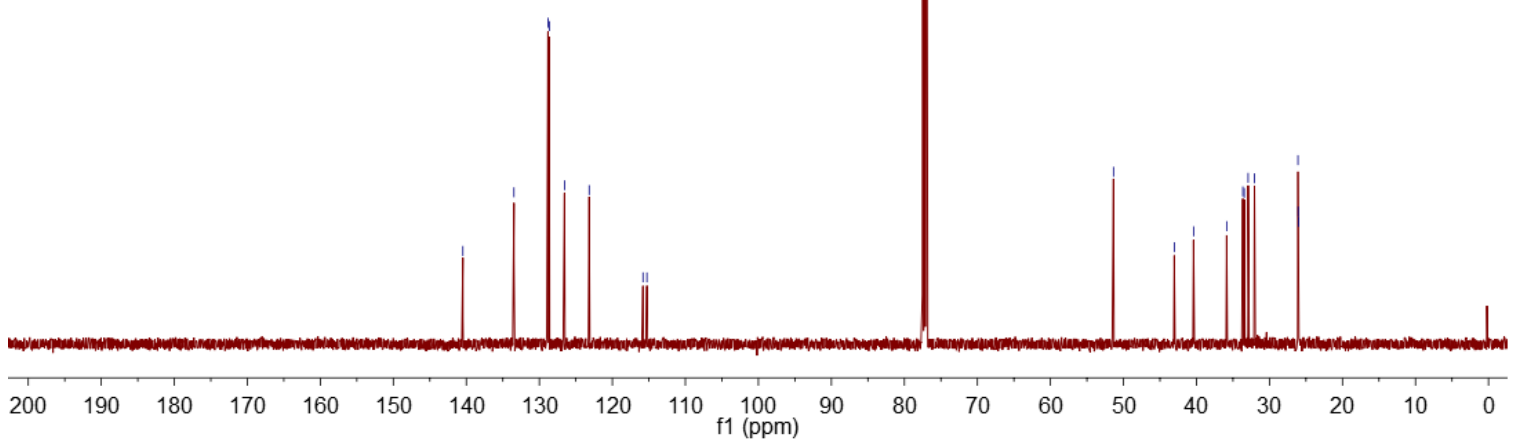


$400 \mathrm{MHz}, \mathrm{CDCl}_{3}$

[3ai] דำ<smiles>C=CCC(C)(C#N)[C@@H](C=C)CCc1ccccc1</smiles>

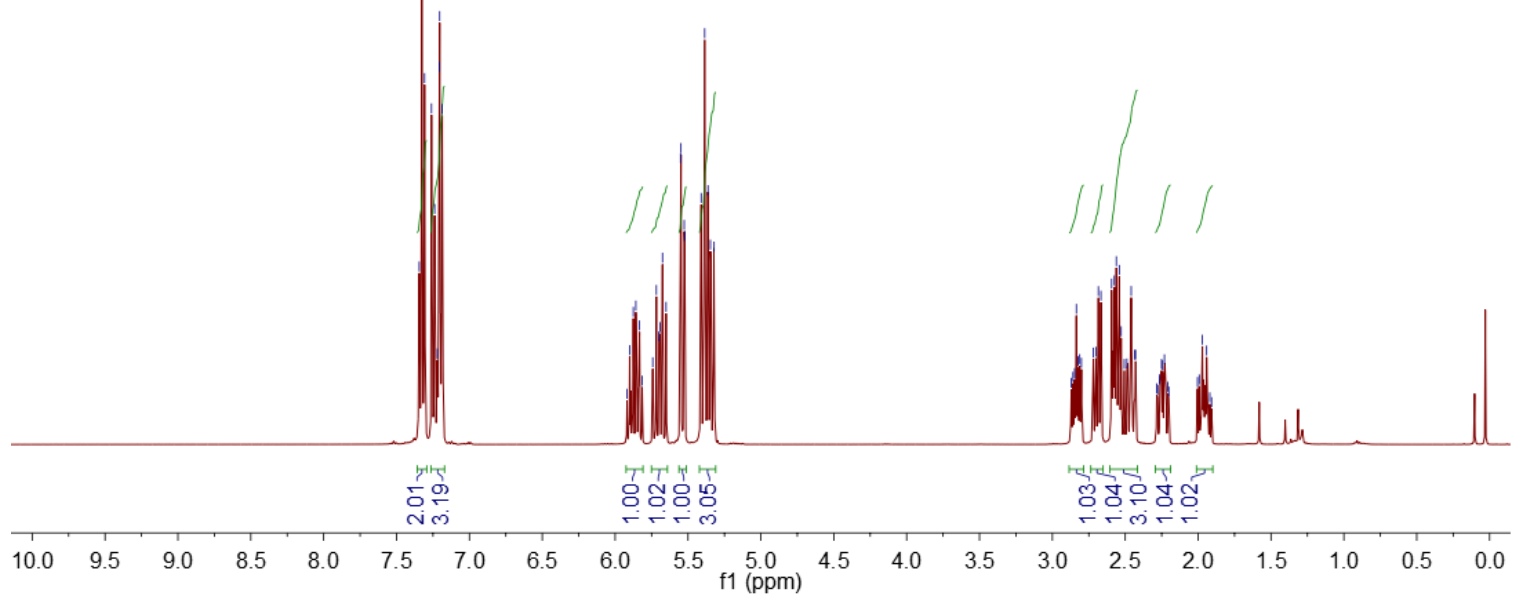
$101 \mathrm{MHz}, \mathrm{CDCl}_{3}$

[3ai]

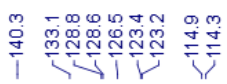<smiles>C=CCC(C#N)(C#N)C(C=C)CCc1ccccc1</smiles>

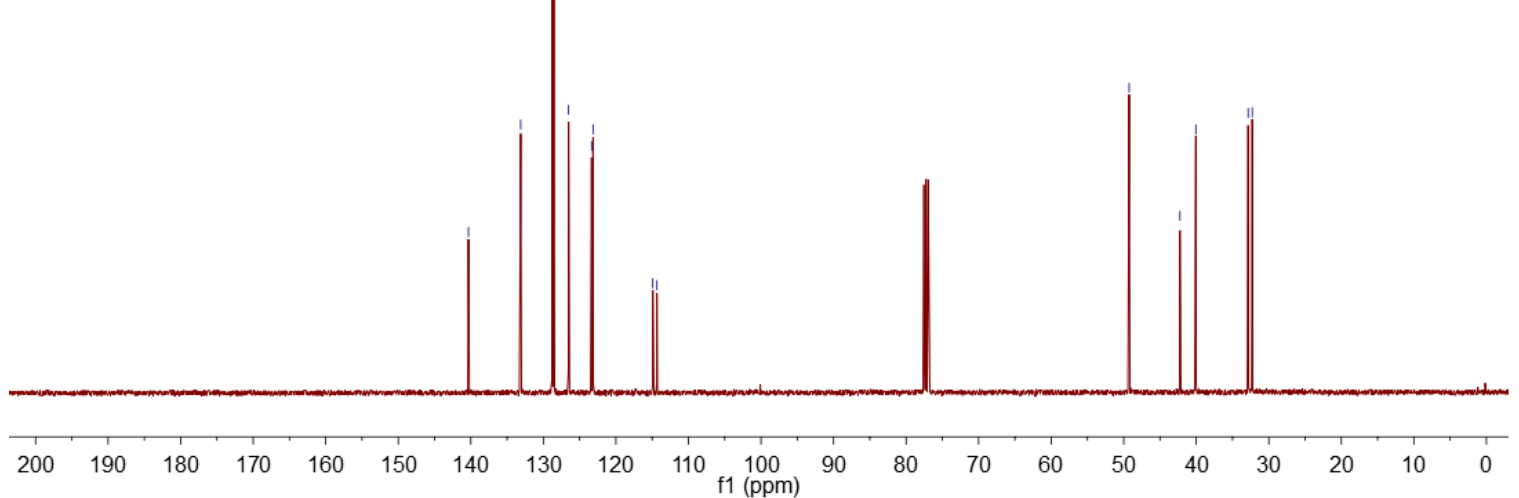


$400 \mathrm{MHz}, \mathrm{CDCl}_{3}$

[3aj]<smiles>C#CCC(C#N)(C#N)[C@H](C=C)CCc1ccccc1</smiles>

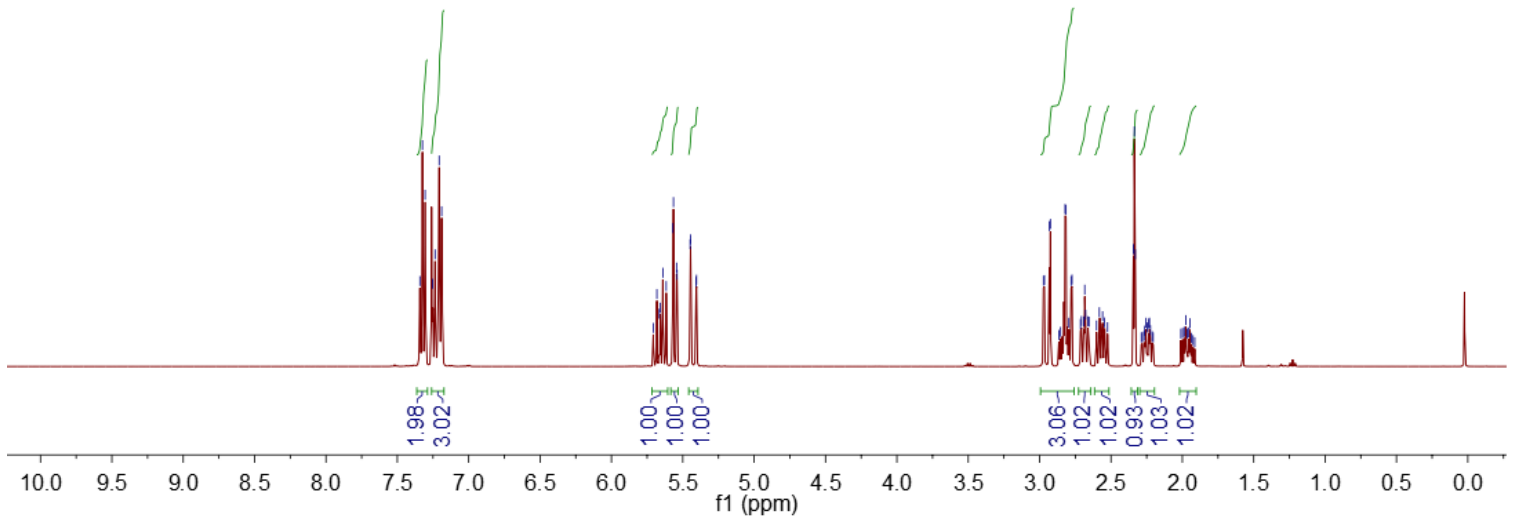

$126 \mathrm{MHz}, \mathrm{CDCl}_{3}$

[3aj]

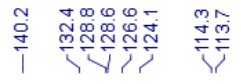

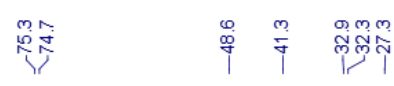<smiles>C#CCC(C#N)(C#N)[C@@H](C=C)CCc1ccccc1</smiles>

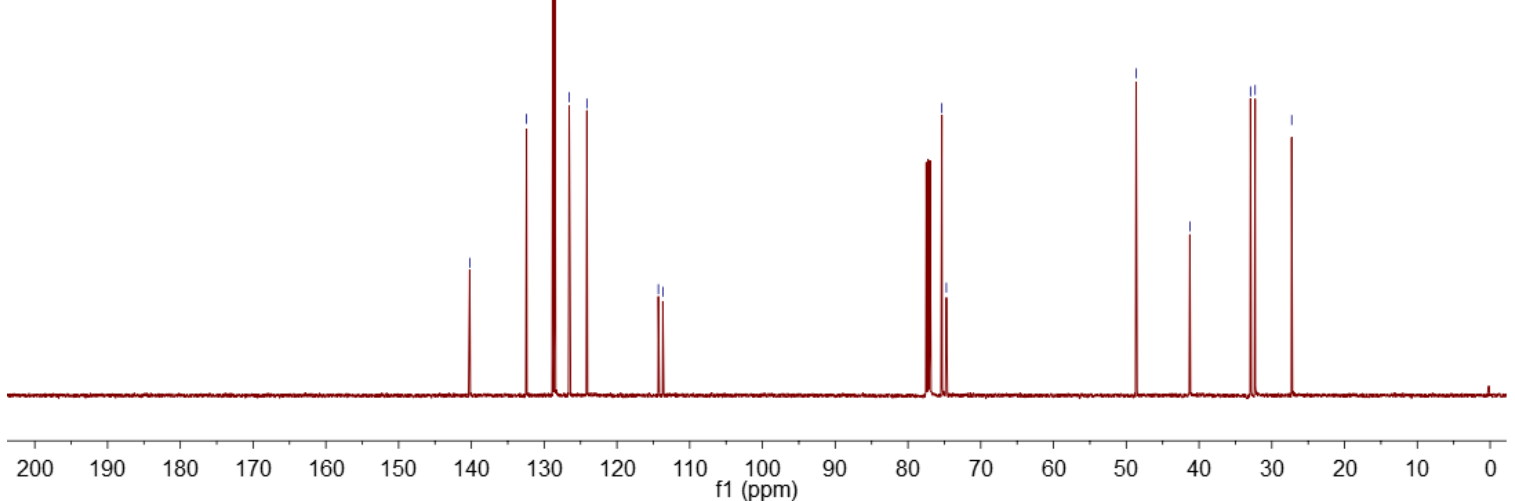


$500 \mathrm{MHz}, \mathrm{CDCl}_{3}$

[3ak]<smiles>C=C[C@H](CCc1ccccc1)C(C#N)C(C)=O</smiles>

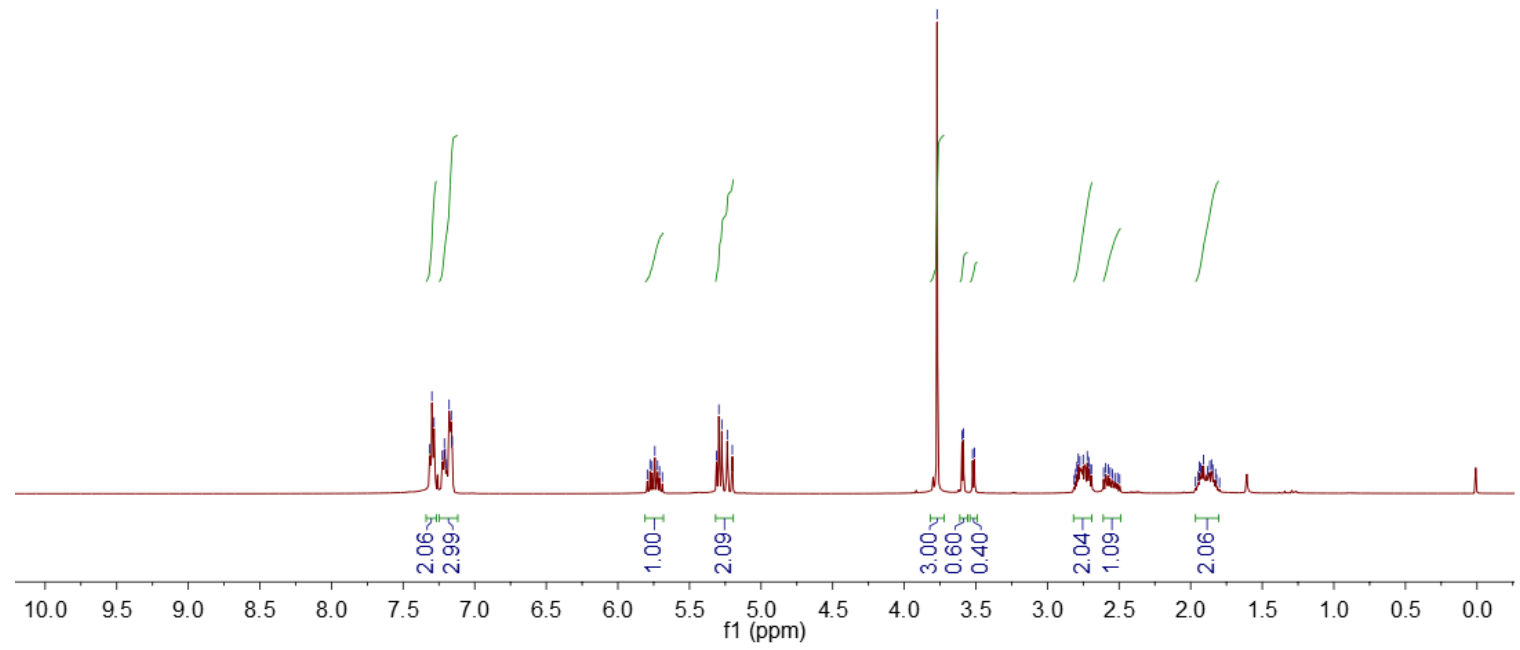

$126 \mathrm{MHz}, \mathrm{CDCl}_{3}$

[3ak]

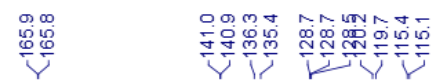

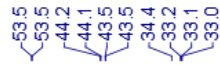<smiles>C=C[C@H](CCc1ccccc1)C(C#N)C(C)=O</smiles>

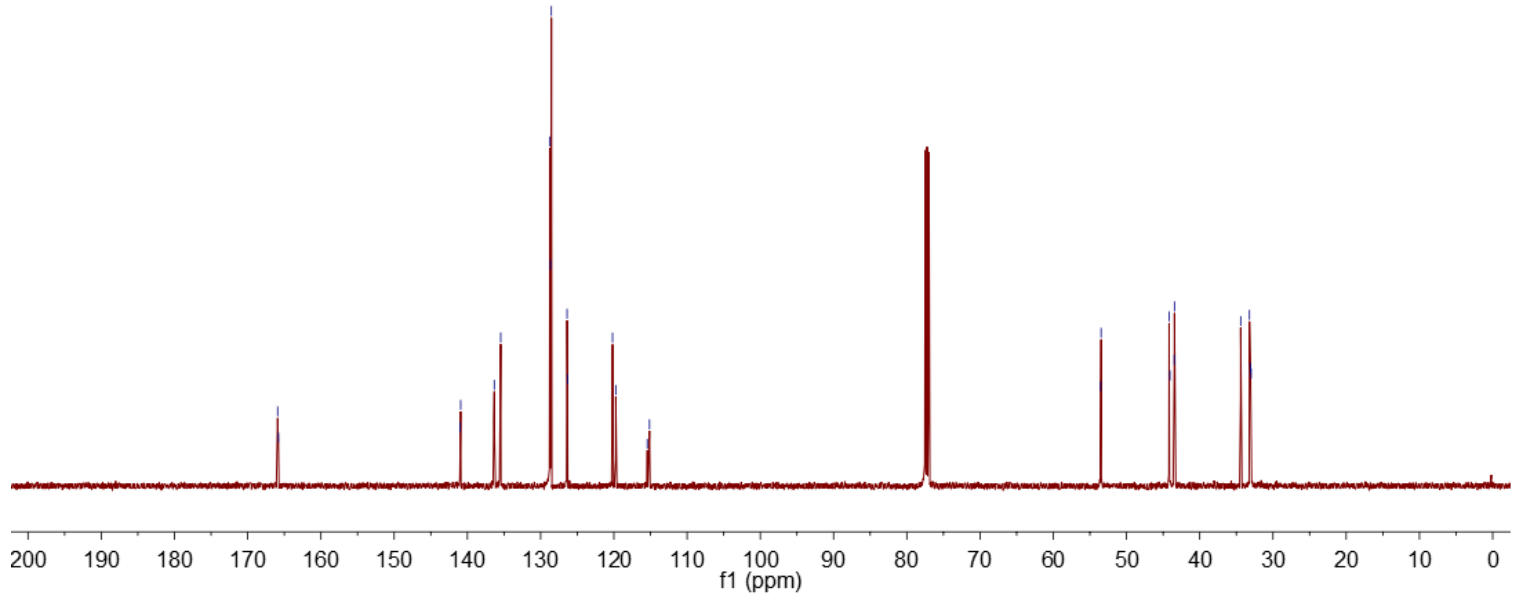


$500 \mathrm{MHz}, \mathrm{CDCl}_{3}$

[3al]

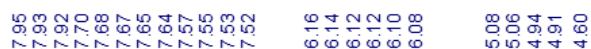

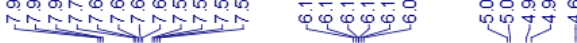

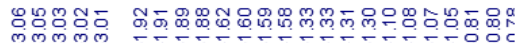

लूलm एT,<smiles>C=C[C@H](CCc1ccccc1)C(S(=O)Oc1ccccc1)S(=O)(=O)c1ccccc1</smiles>

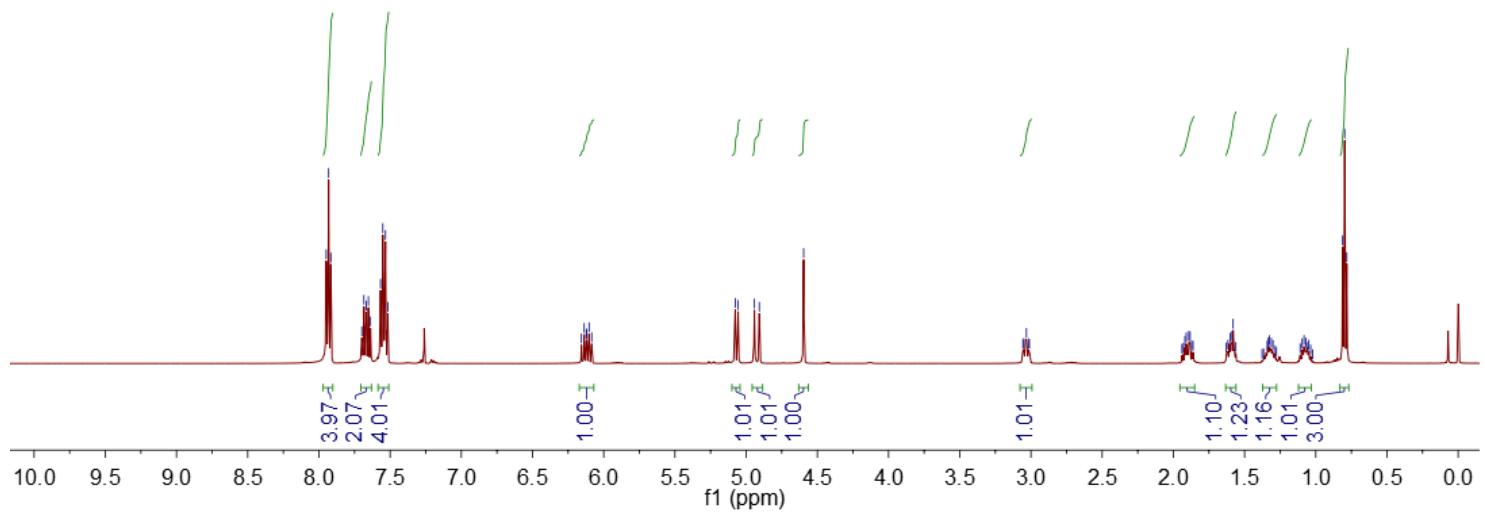

$126 \mathrm{MHz}, \mathrm{CDCl}_{3}$

[3al]

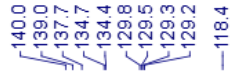<smiles>C=CC(CCc1ccccc1)C(S(=O)(=O)Oc1ccccc1)S(=O)(=O)c1ccccc1</smiles>

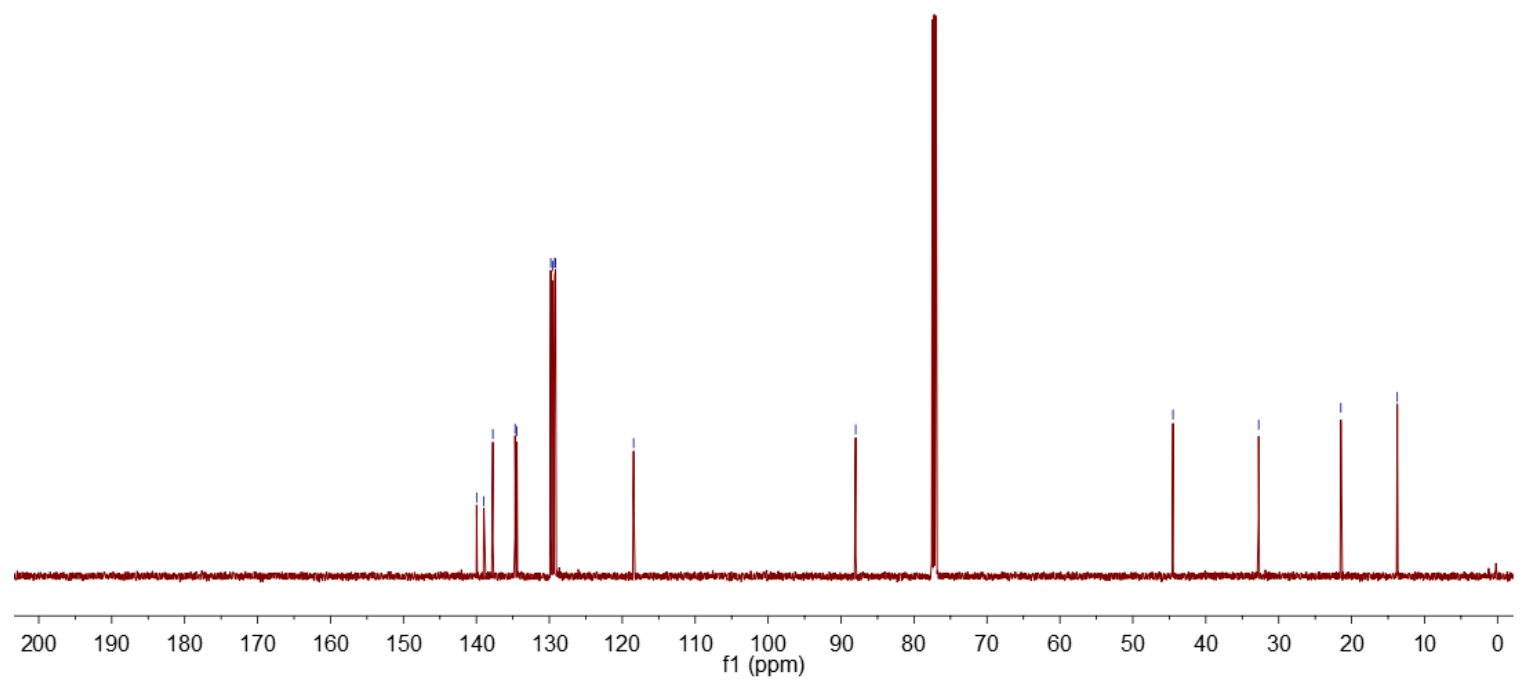


$500 \mathrm{MHz}, \mathrm{CDCl}_{3}$

[3bb]

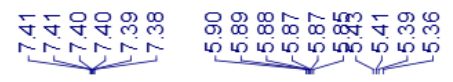

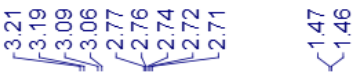<smiles>C=CC(C)C(C#N)(C#N)Cc1ccccc1</smiles>

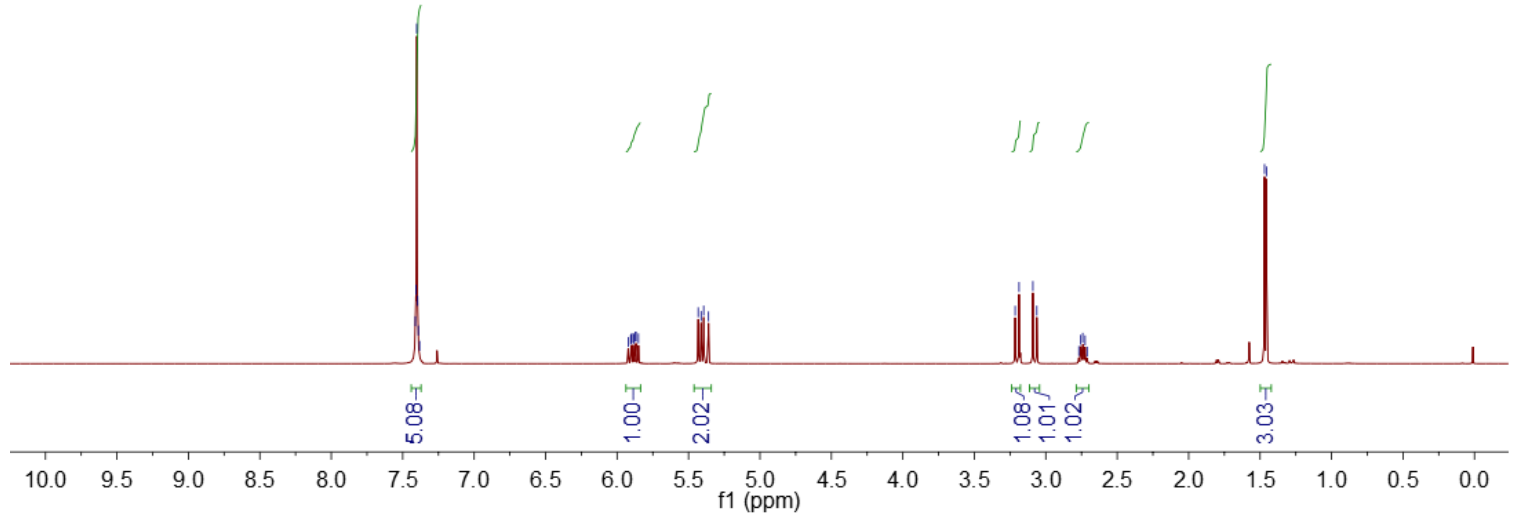

$126 \mathrm{MHz}, \mathrm{CDCl}_{3}$

[3bb]

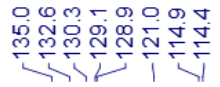

每
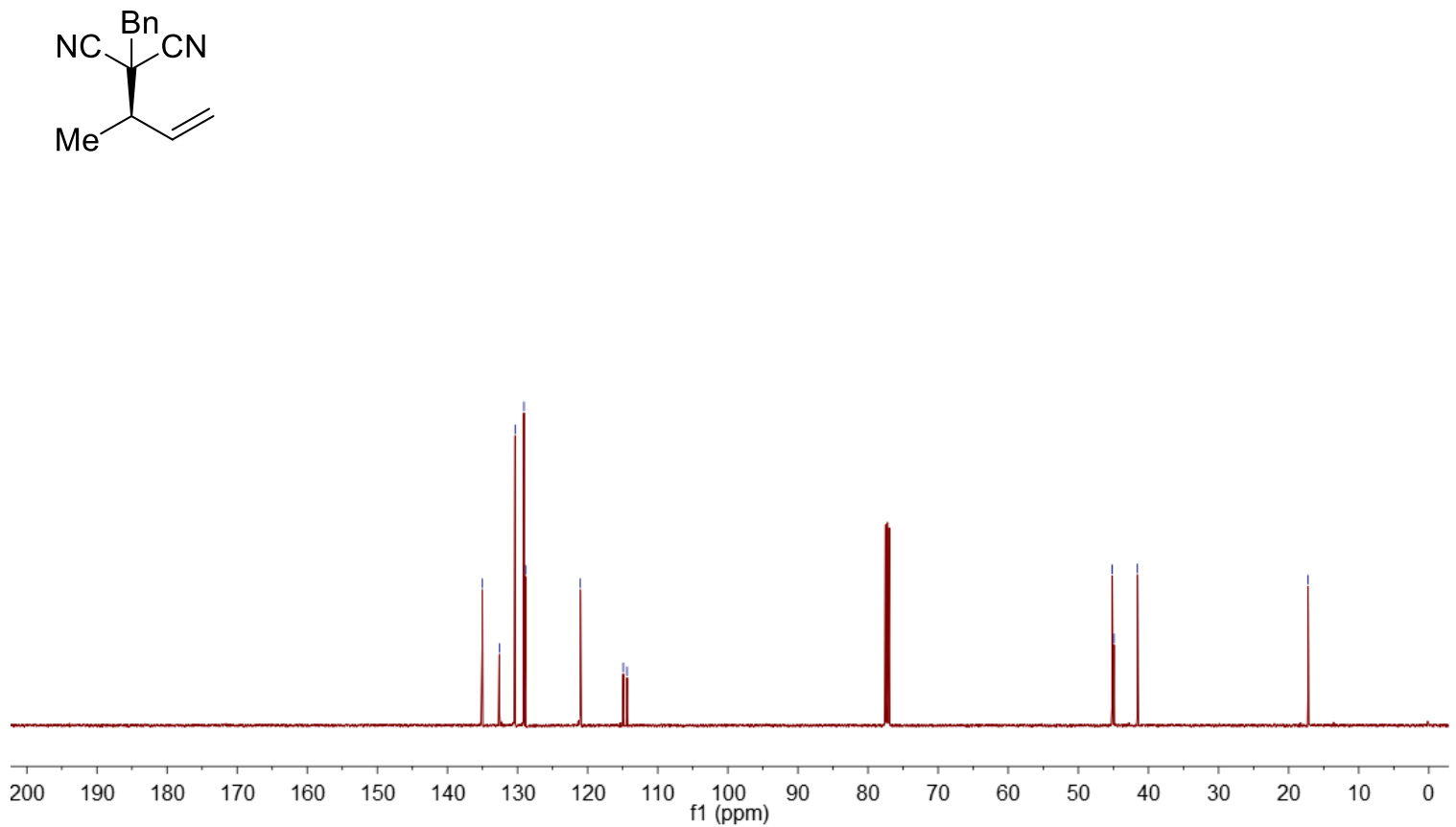
$400 \mathrm{MHz}, \mathrm{CDCl}_{3}$

[3cb]<smiles>C=C[C@@H](CCC)C(Br)(C#N)C#N</smiles>

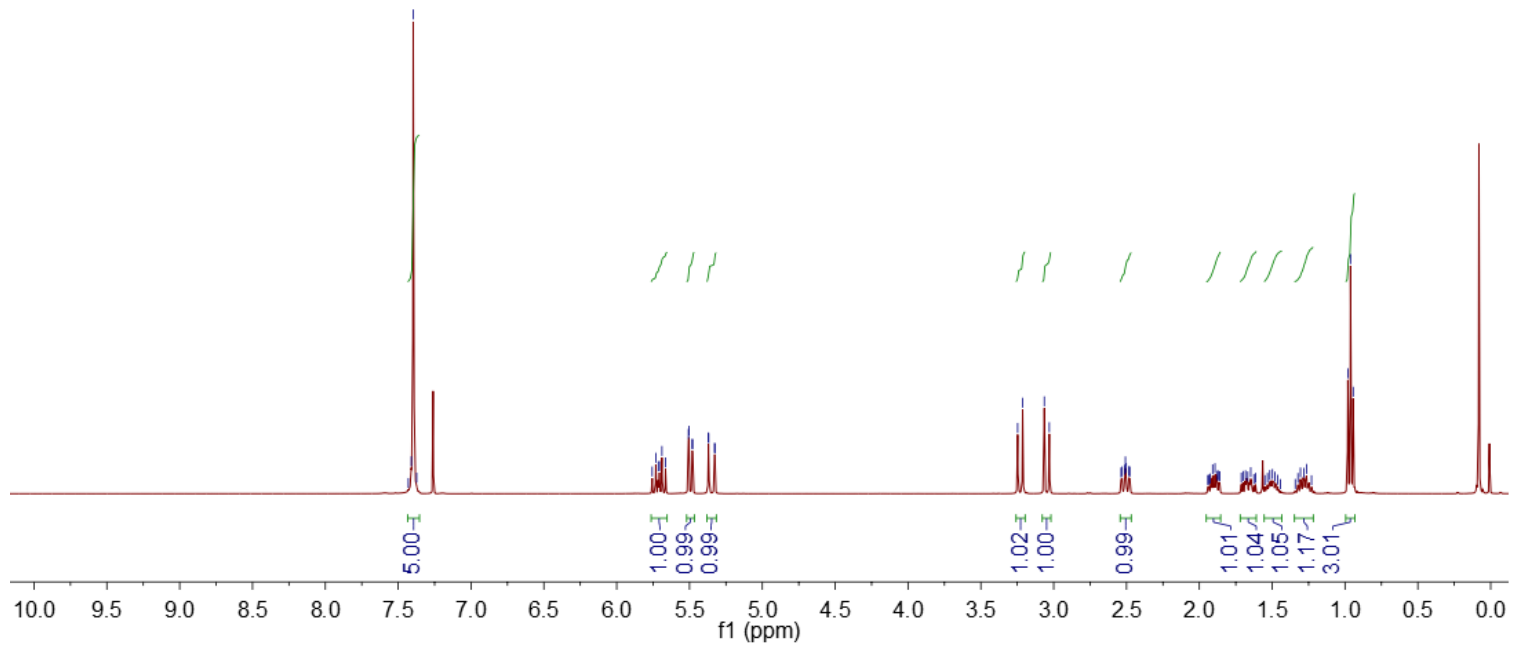

$126 \mathrm{MHz}, \mathrm{CDCl}_{3}$

[3cb]<smiles>C=C[C@@H](CCC)C(Br)(C#N)C#N</smiles>

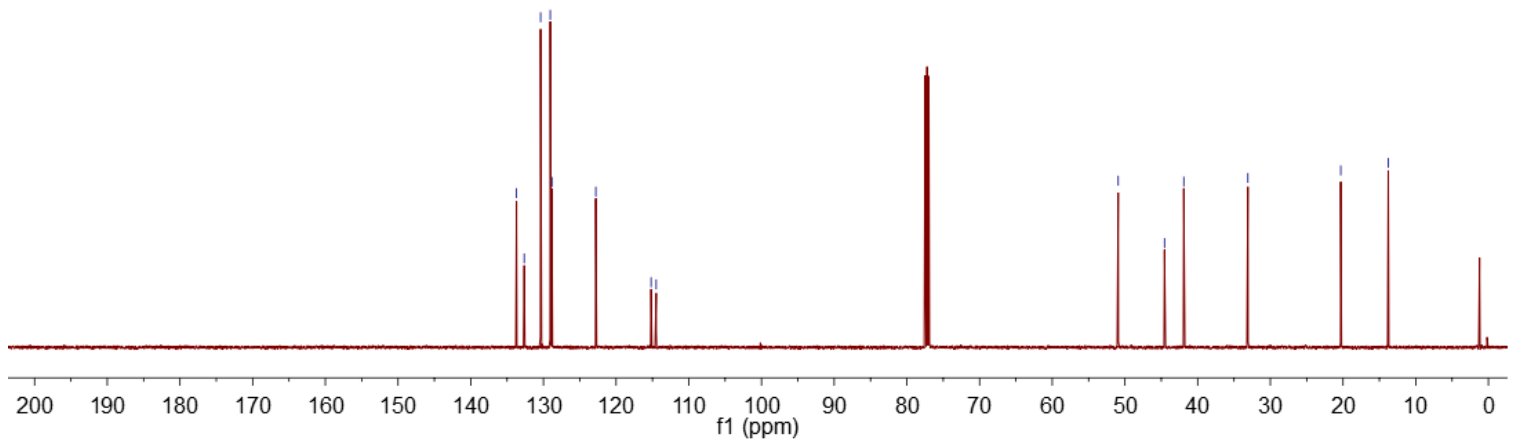


$400 \mathrm{MHz}, \mathrm{CDCl}_{3}$

[3db]

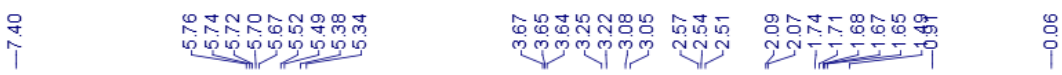<smiles>C=C[C@@H](CCCO[SbH3])C(C#N)(C#N)Cc1ccccc1</smiles>

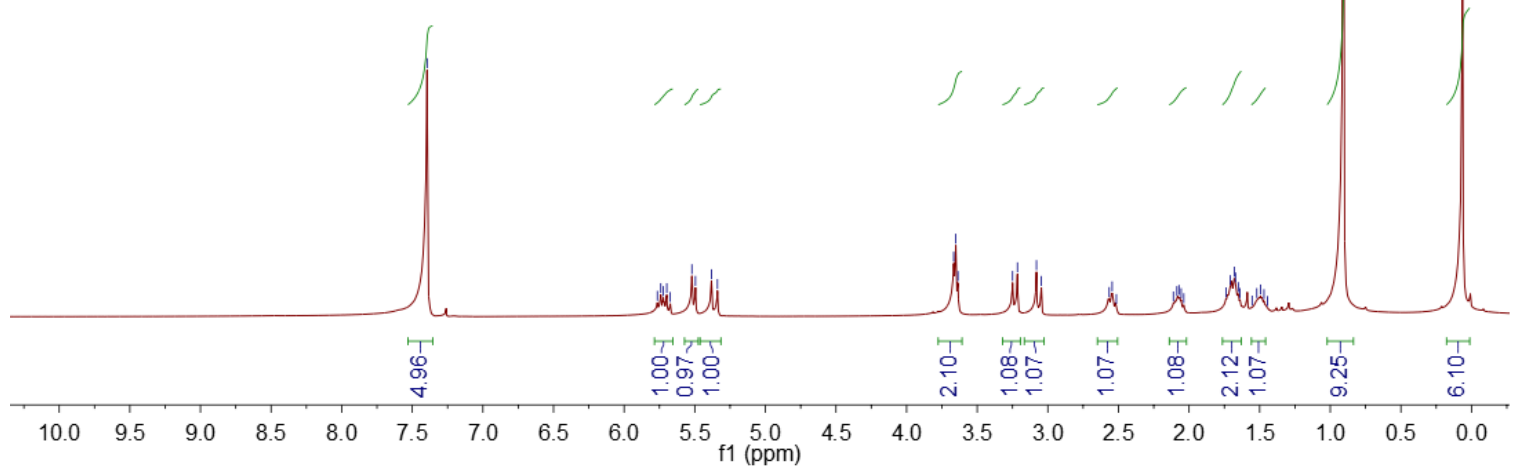

$126 \mathrm{MHz}, \mathrm{CDCl}_{3}$

[3db]

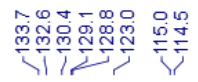

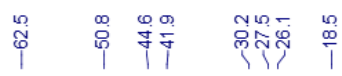
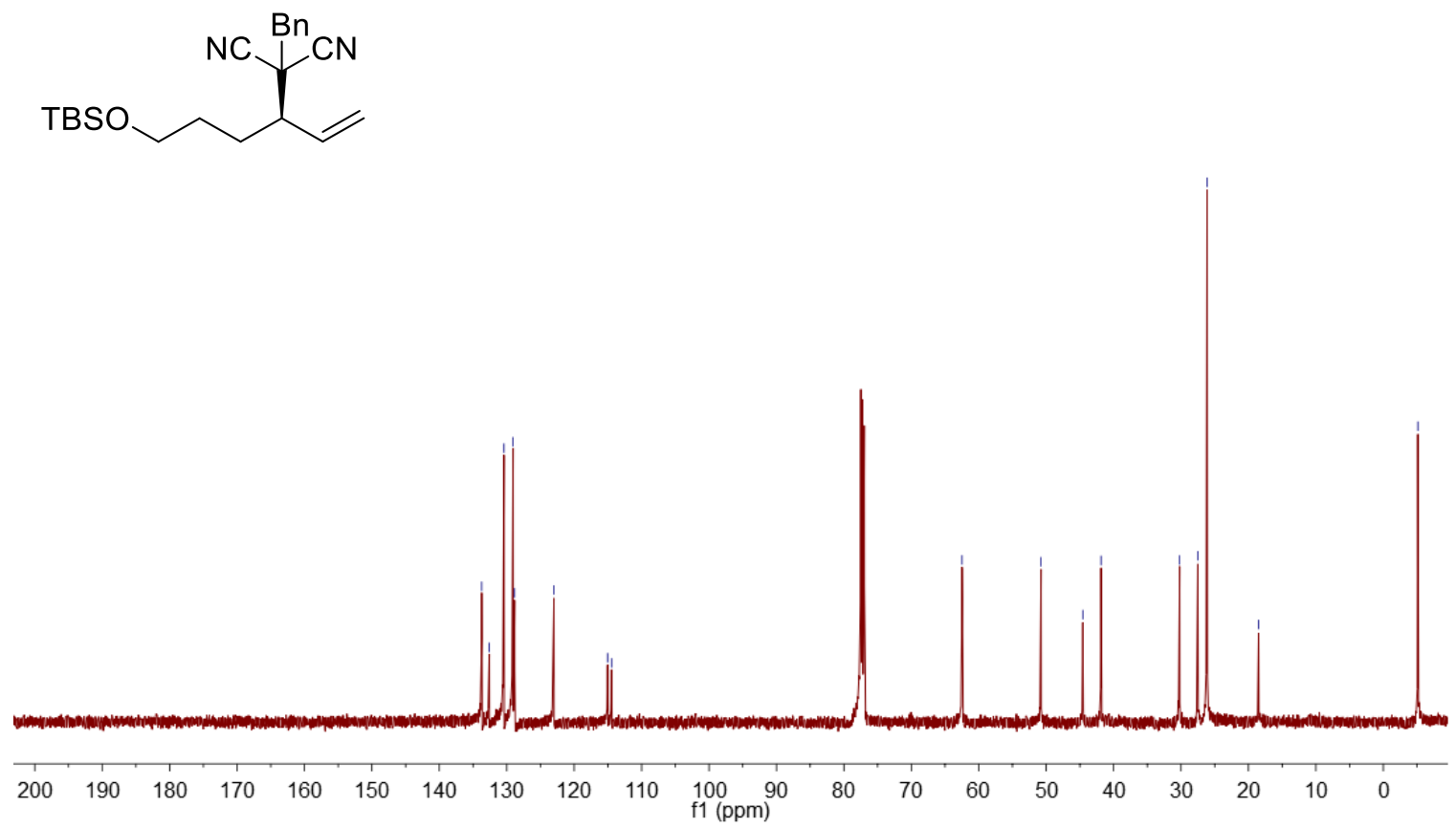
$500 \mathrm{MHz}, \mathrm{CDCl}_{3}$

[3eb]

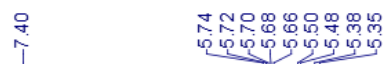

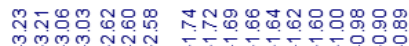

mmm aㅐ
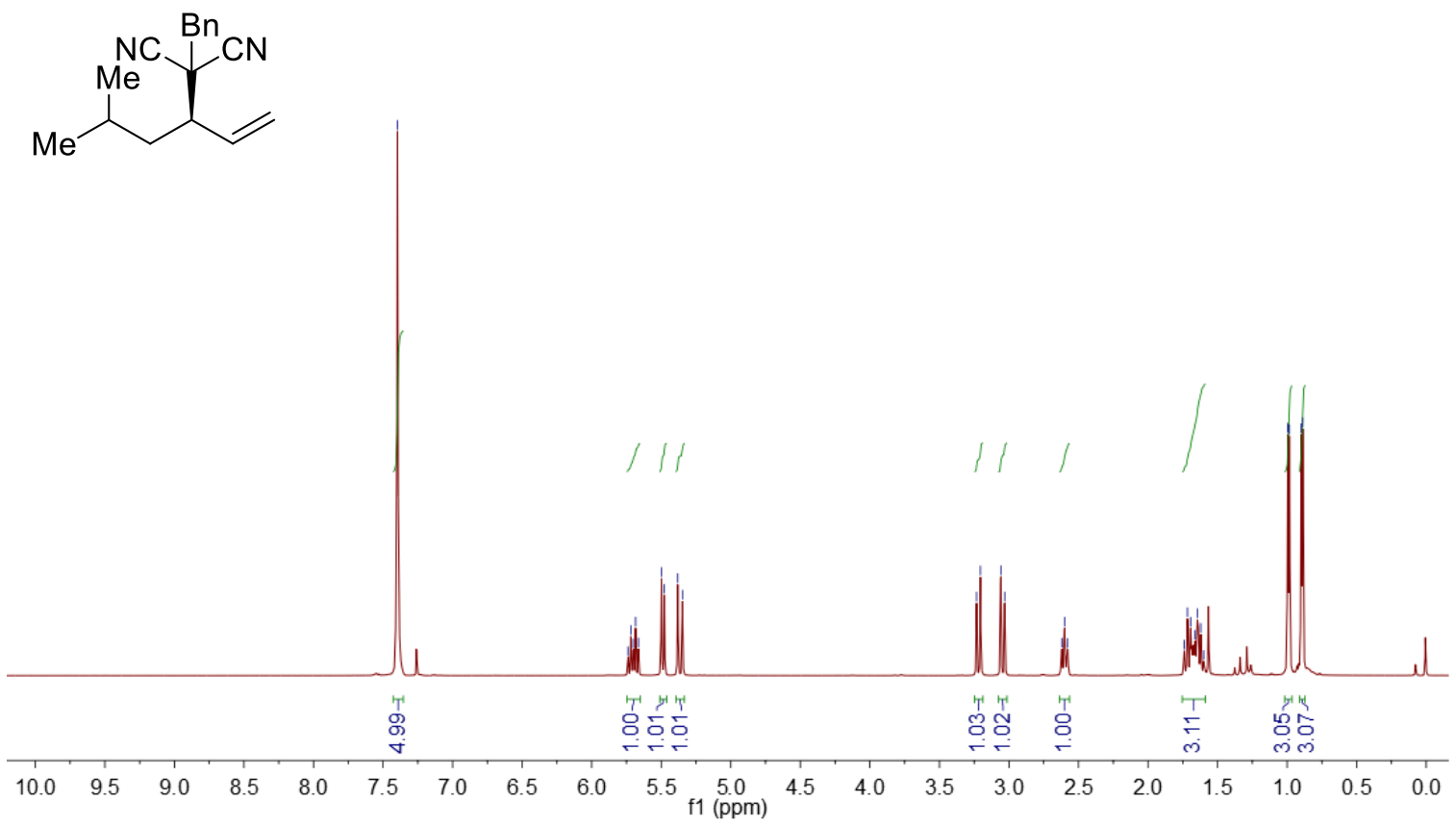

$126 \mathrm{MHz}, \mathrm{CDCl}_{3}$

[3eb]<smiles>C=C[C@@H](CC(C)C)C(C)(C)CBr</smiles>

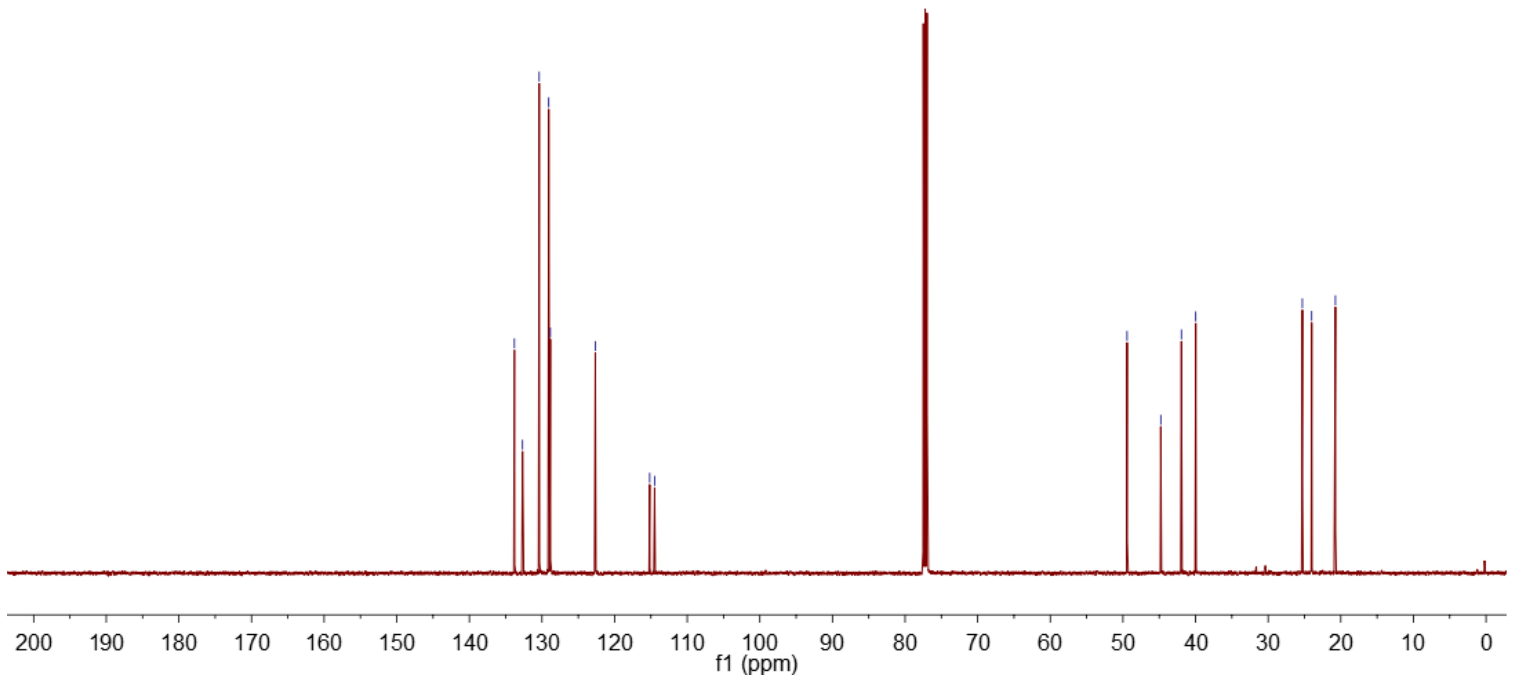


$400 \mathrm{MHz}, \mathrm{CDCl}_{3}$

[3fb]<smiles>C=C[C@@H](C1CC1)C(Br)(C#N)C#N</smiles>

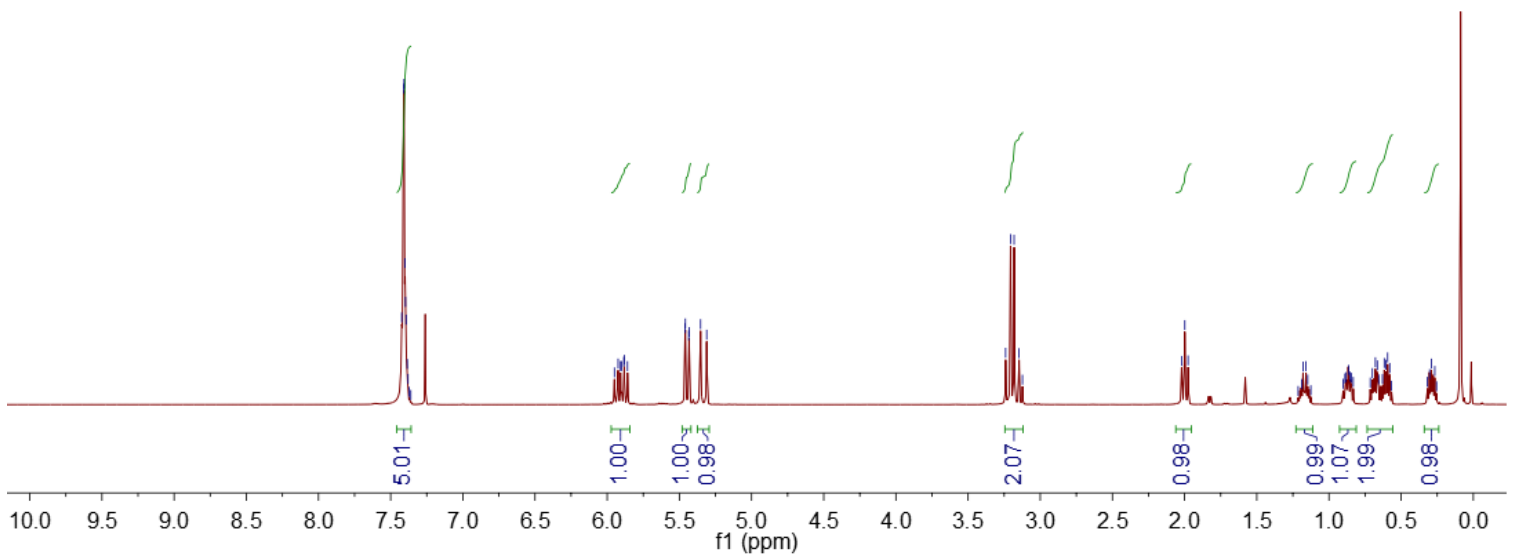

$126 \mathrm{MHz}, \mathrm{CDCl}_{3}$

[3fb]<smiles>C=CC(C1CC1)C(C#N)(C#N)Cc1ccccc1</smiles>

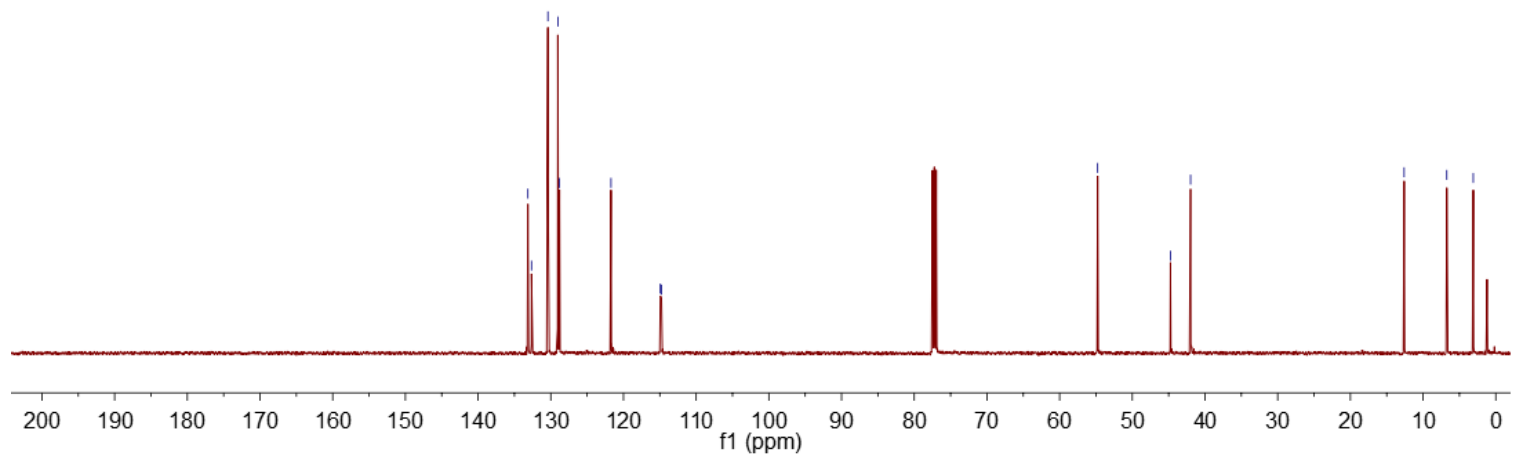


$400 \mathrm{MHz}, \mathrm{CDCl}_{3}$

[3gb]

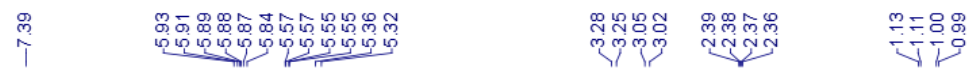

Me

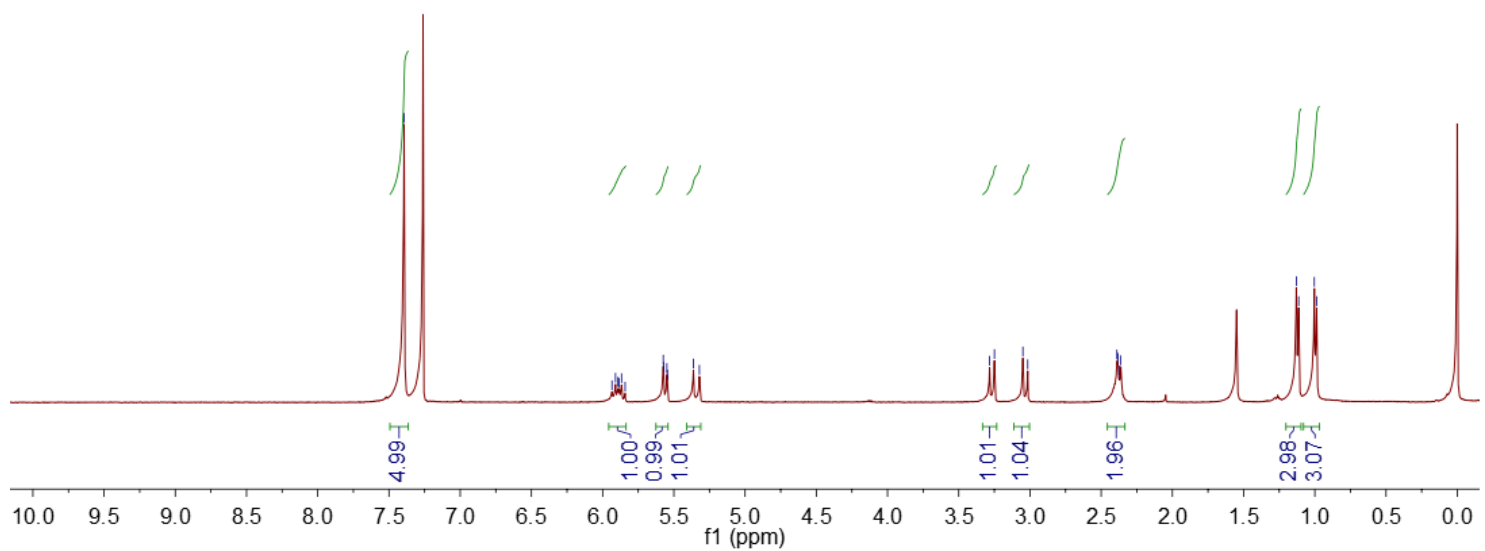

$126 \mathrm{MHz}, \mathrm{CDCl}_{3}$

[3gb]<smiles>C=C[C@@H](C(C)C)C(Br)(C#N)C#N</smiles>

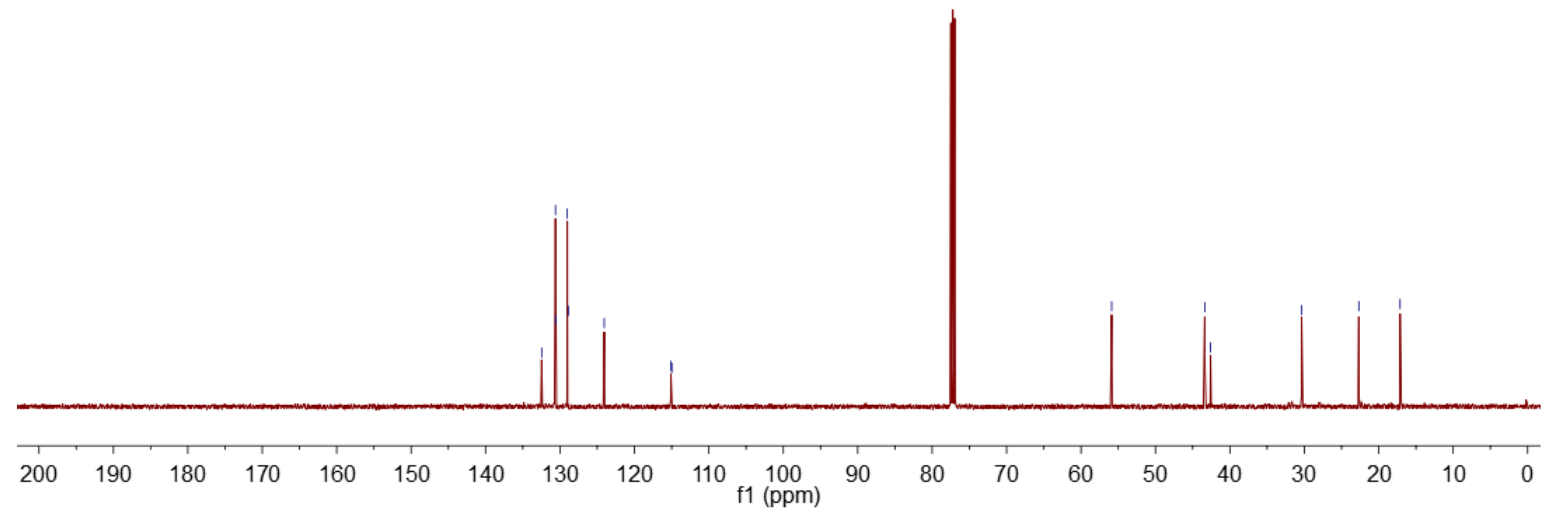


$500 \mathrm{MHz}, \mathrm{CDCl}_{3}$

[3hb]

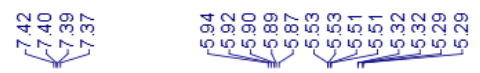

구유요<smiles>C=C[C@@H](C1CCCCC1)C(Br)(C#N)C#N</smiles>
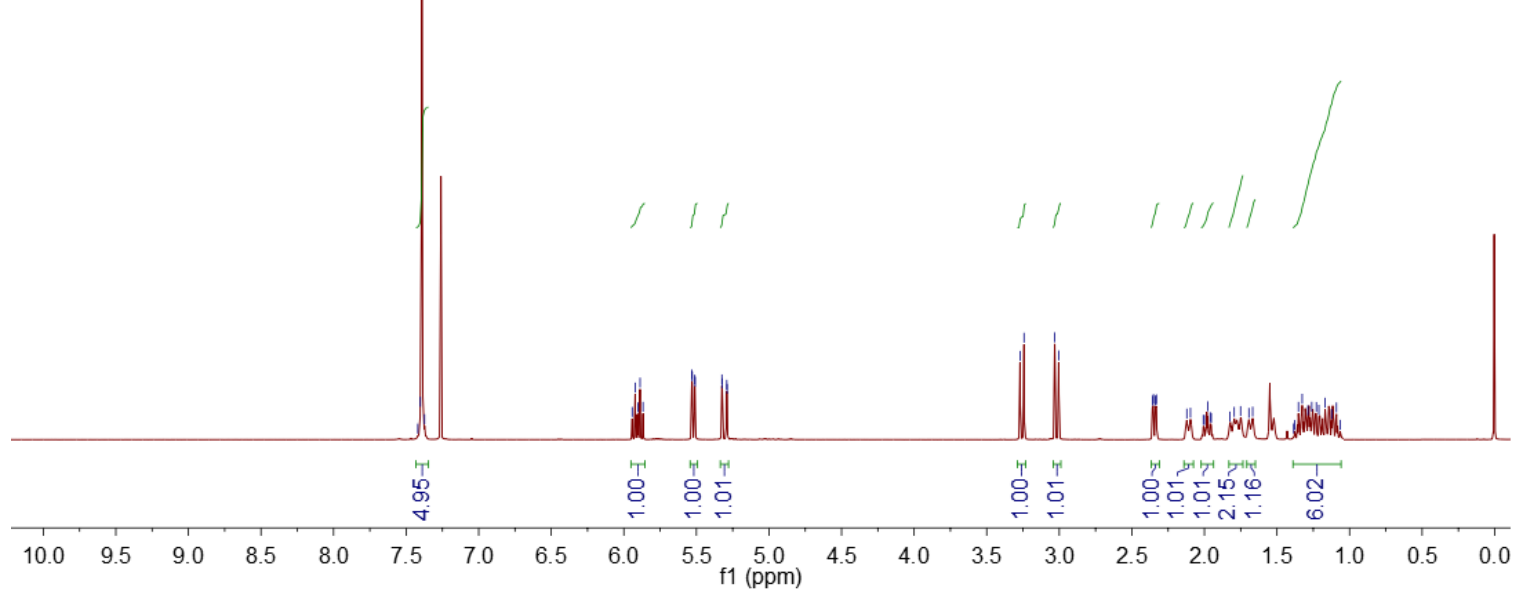

$126 \mathrm{MHz}, \mathrm{CDCl}_{3}$

[3hb]

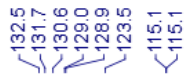

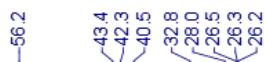<smiles>C=C[C@@H](C1CCCCC1)C(Br)(C#N)C#N</smiles>

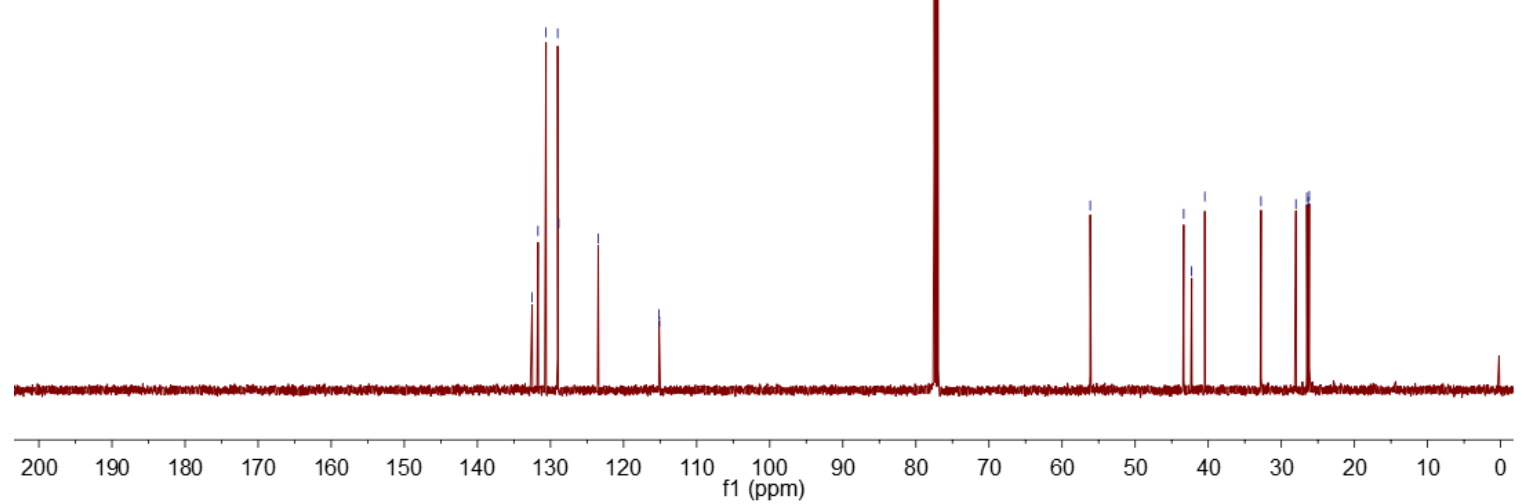


$500 \mathrm{MHz}, \mathrm{CDCl}_{3}$

[3ib]

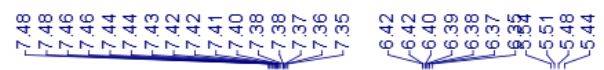

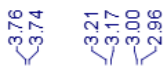<smiles>C=C[C@@H](c1ccccc1)C(Br)(C#N)C#N</smiles>
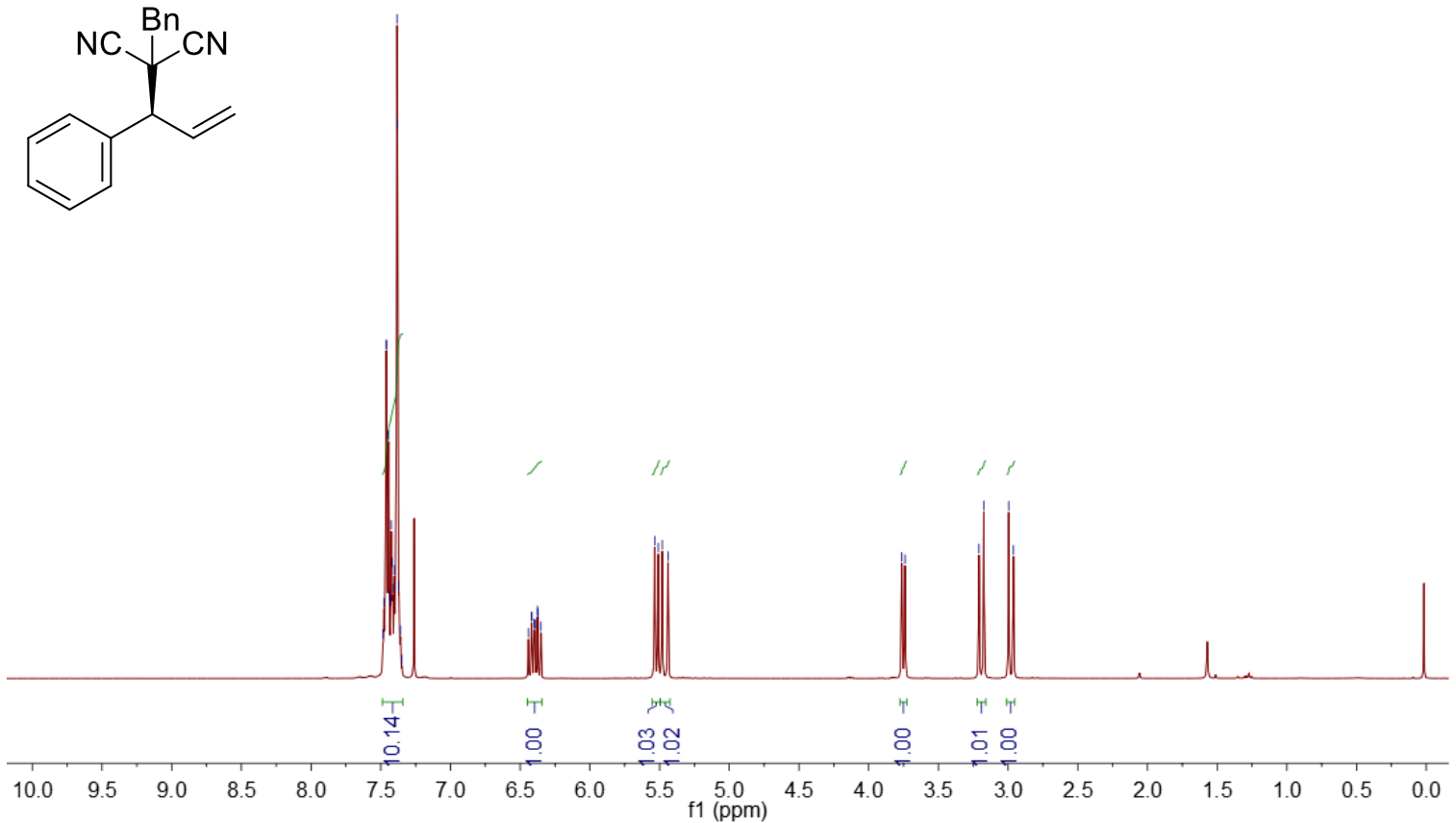

$126 \mathrm{MHz}, \mathrm{CDCl}_{3}$

[3ib]

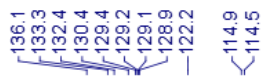

䨌<smiles>C=C[C@@H](c1ccccc1)C(Br)(C#N)C#N</smiles>

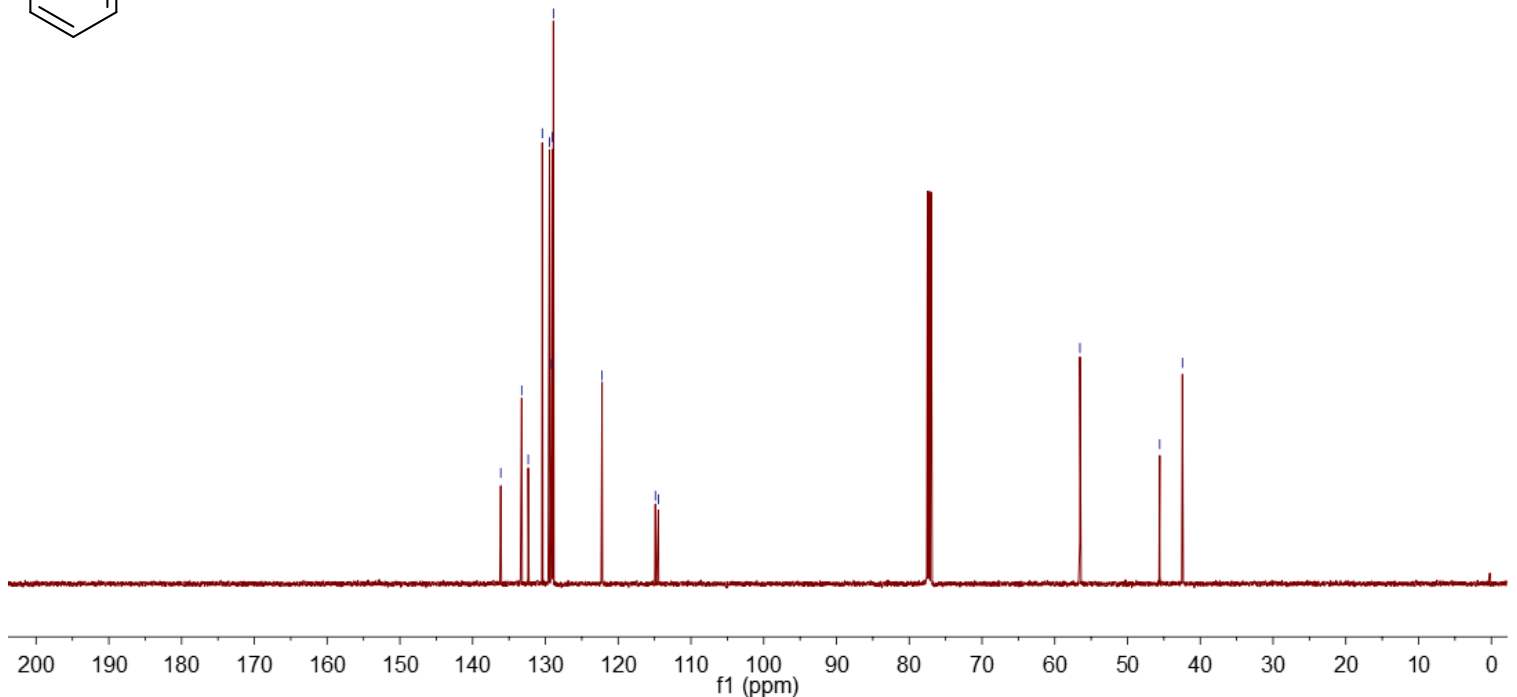


$400 \mathrm{MHz}, \mathrm{CDCl}_{3}$

[3ja]

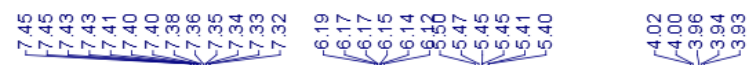<smiles>C=C[C@H](c1ccccc1)C(C#N)C#N</smiles>

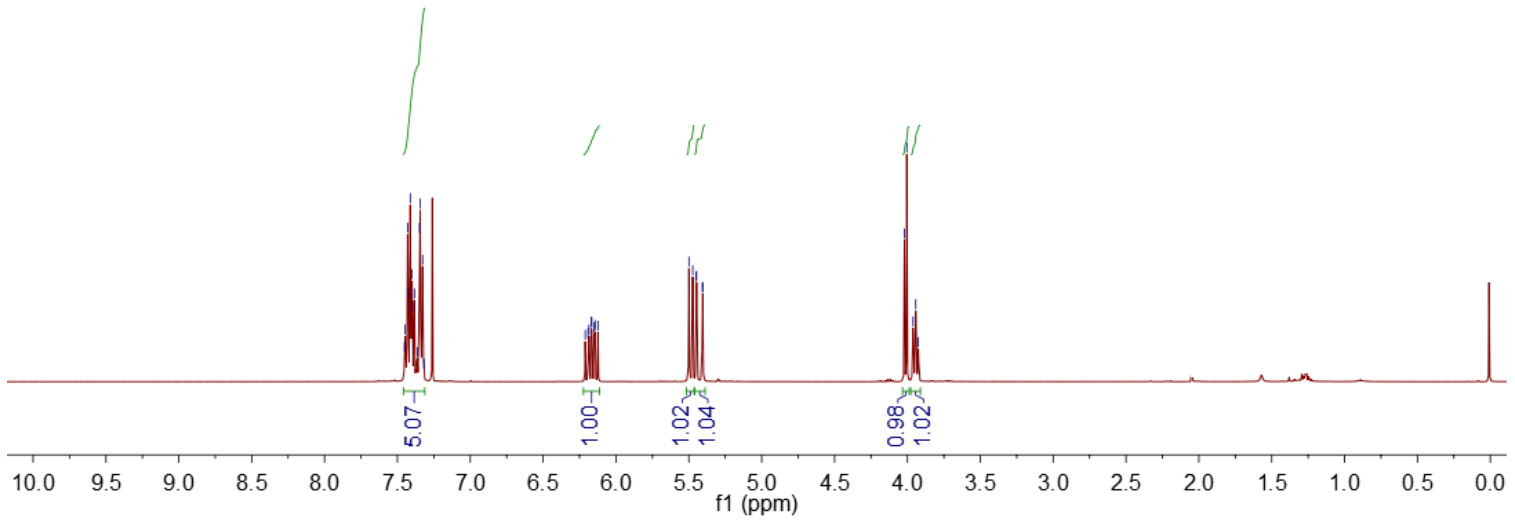

$126 \mathrm{MHz}, \mathrm{CDCl}_{3}$

[3ja]
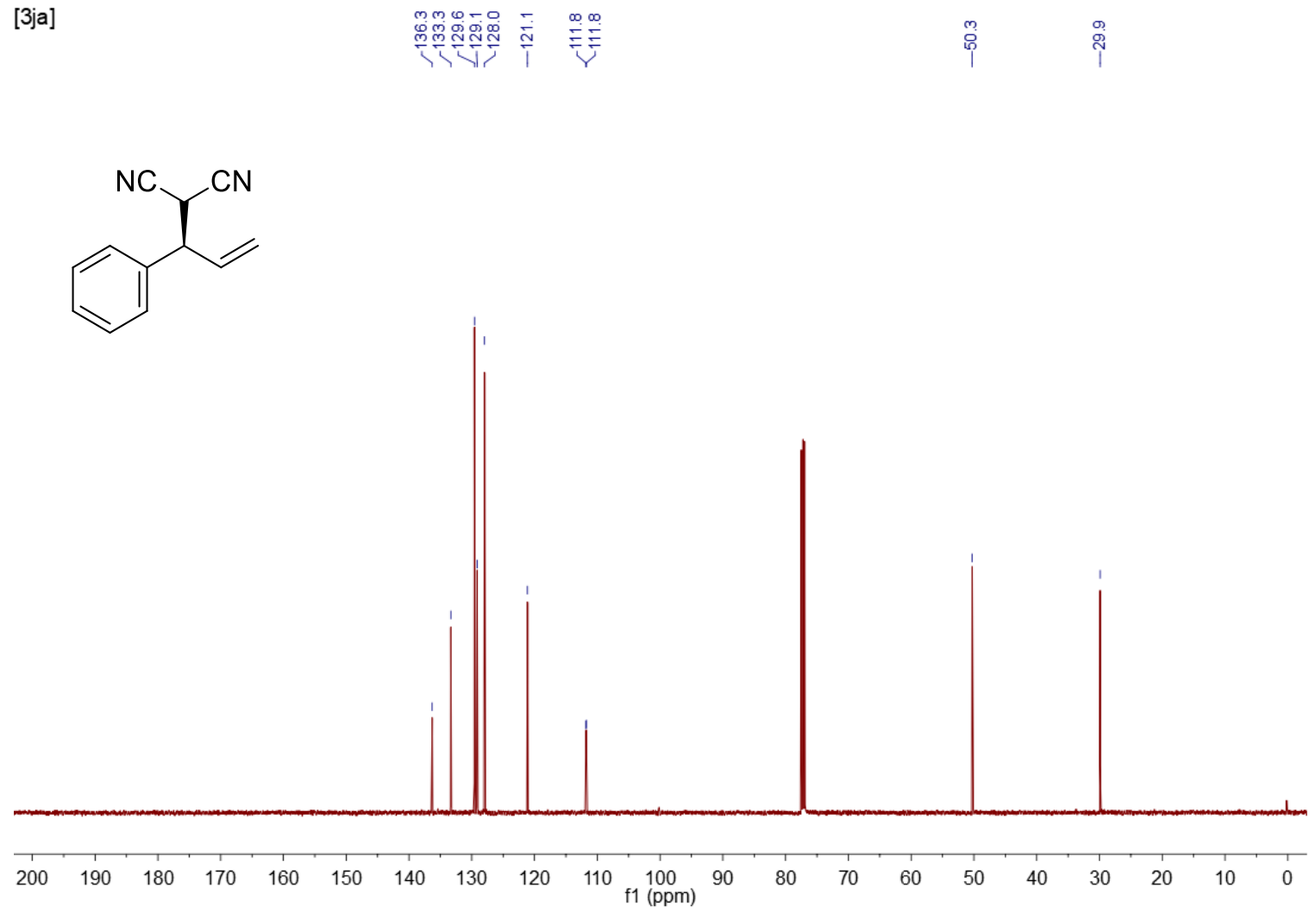
$400 \mathrm{MHz}, \mathrm{CDCl}_{3}$<smiles>C=C[C@H](CO)CCc1ccccc1</smiles>

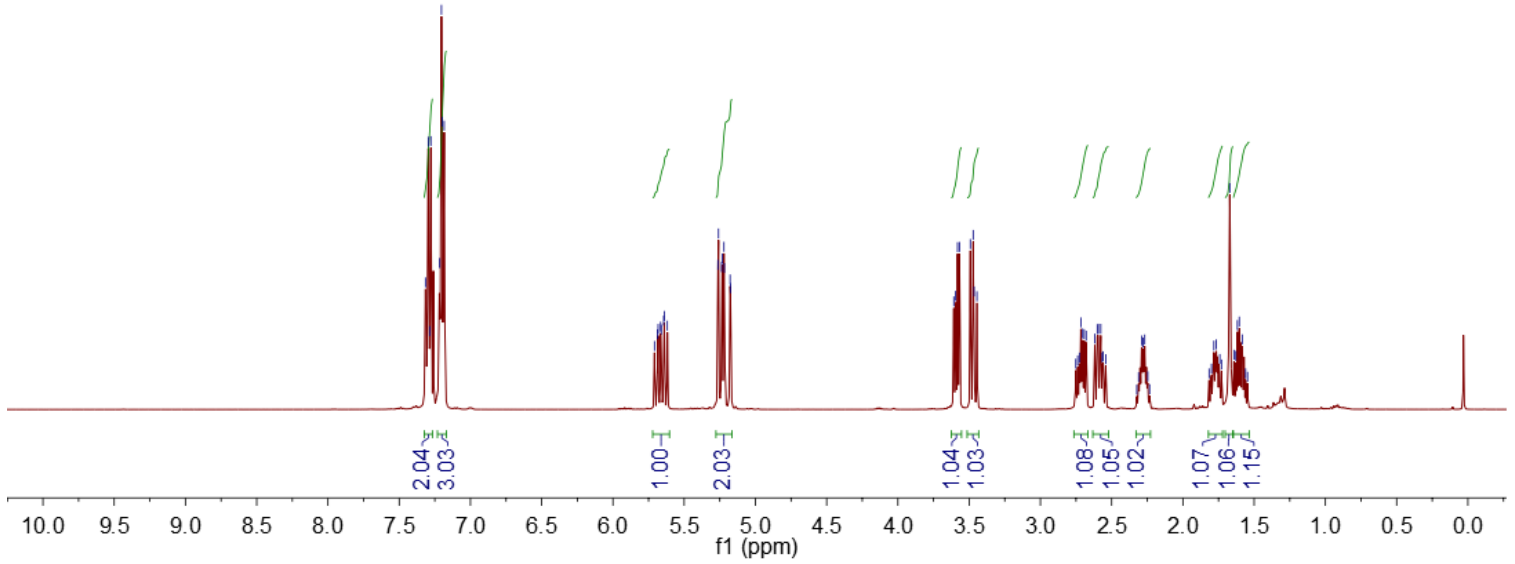

$101 \mathrm{MHz}, \mathrm{CDCl}_{3}$

[4]<smiles>C=C[C@H](CO)CCc1ccccc1</smiles>

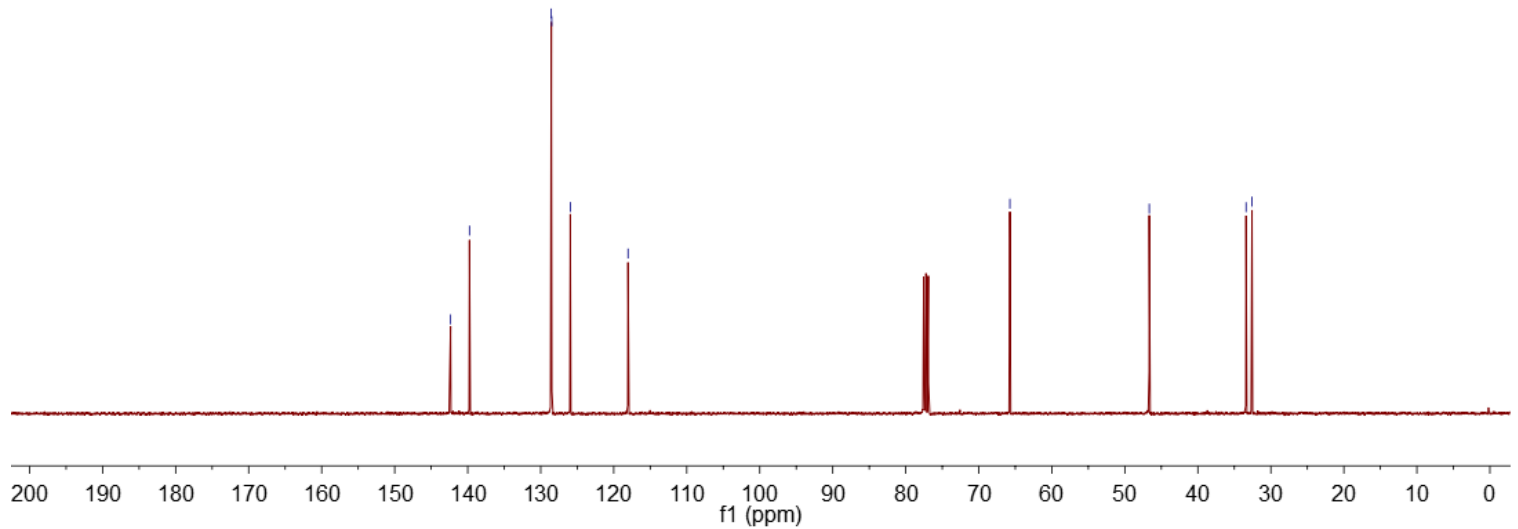


$400 \mathrm{MHz}, \mathrm{CDCl}_{3}$

[5]<smiles>N#CC1(C#N)CC=C[C@@H]1CCc1ccccc1</smiles>

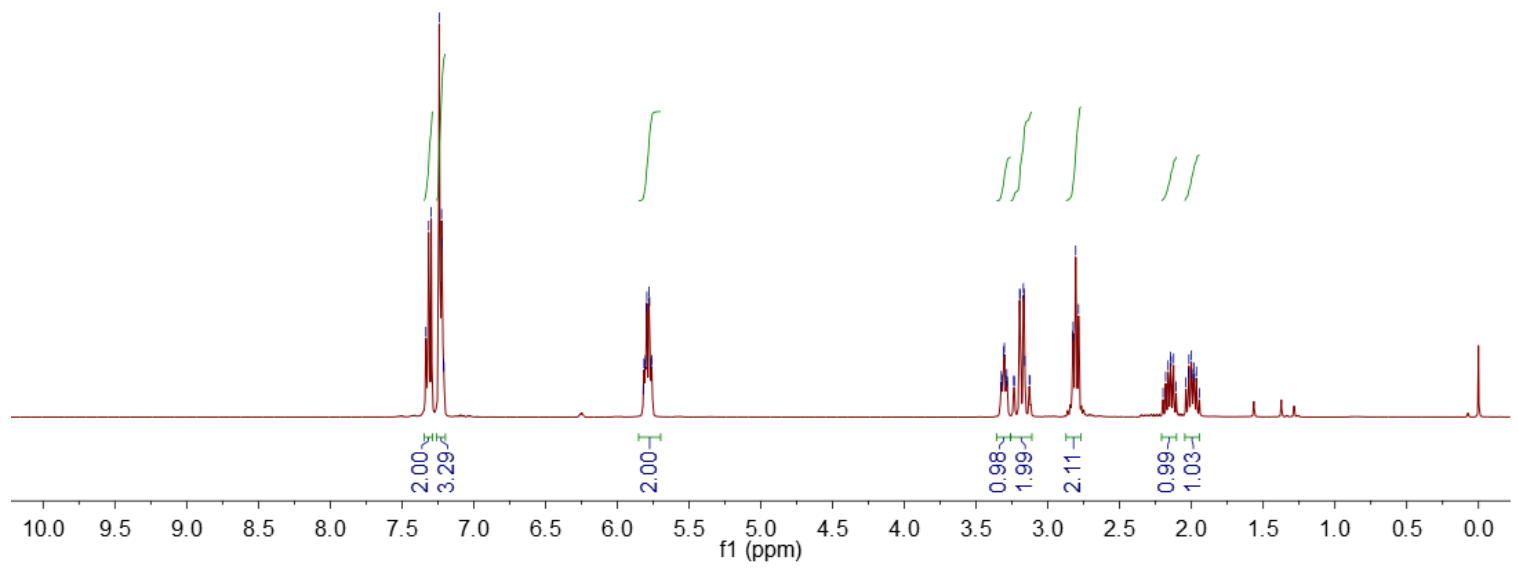

$126 \mathrm{MHz}, \mathrm{CDCl}_{3}$

[5]

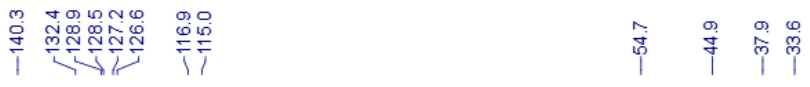<smiles>N#CC1(C#N)CC=CC1CCc1ccccc1</smiles>

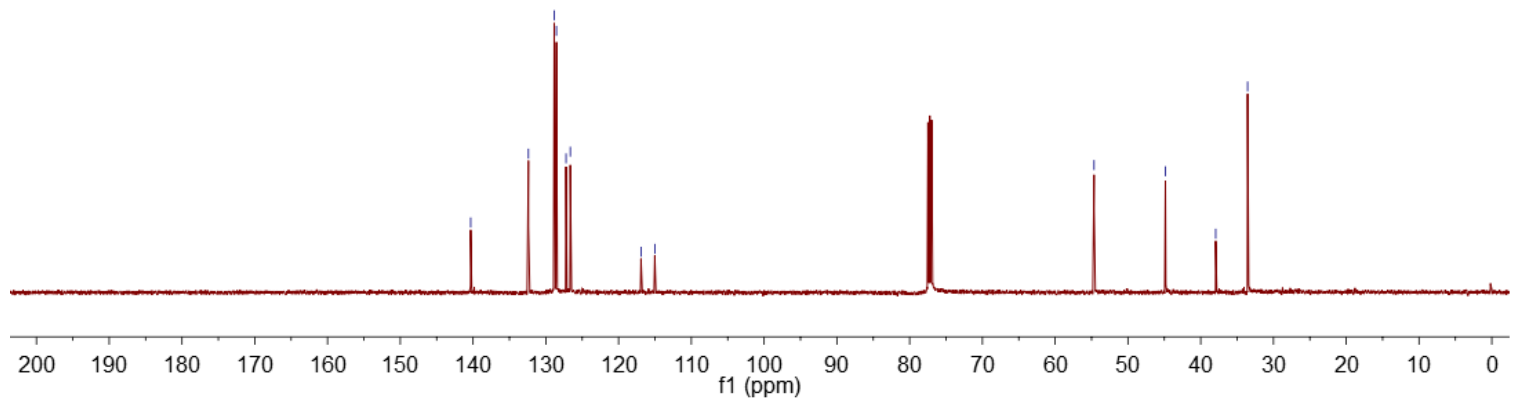


$400 \mathrm{MHz}, \mathrm{CDCl}_{3}$

[6]

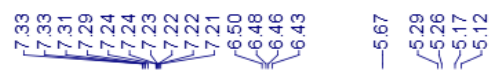

ஜூలి

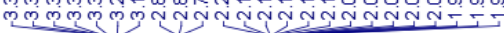<smiles>C=CC1=C[C@@H](CCc2ccccc2)C(C#N)(C#N)C1</smiles>

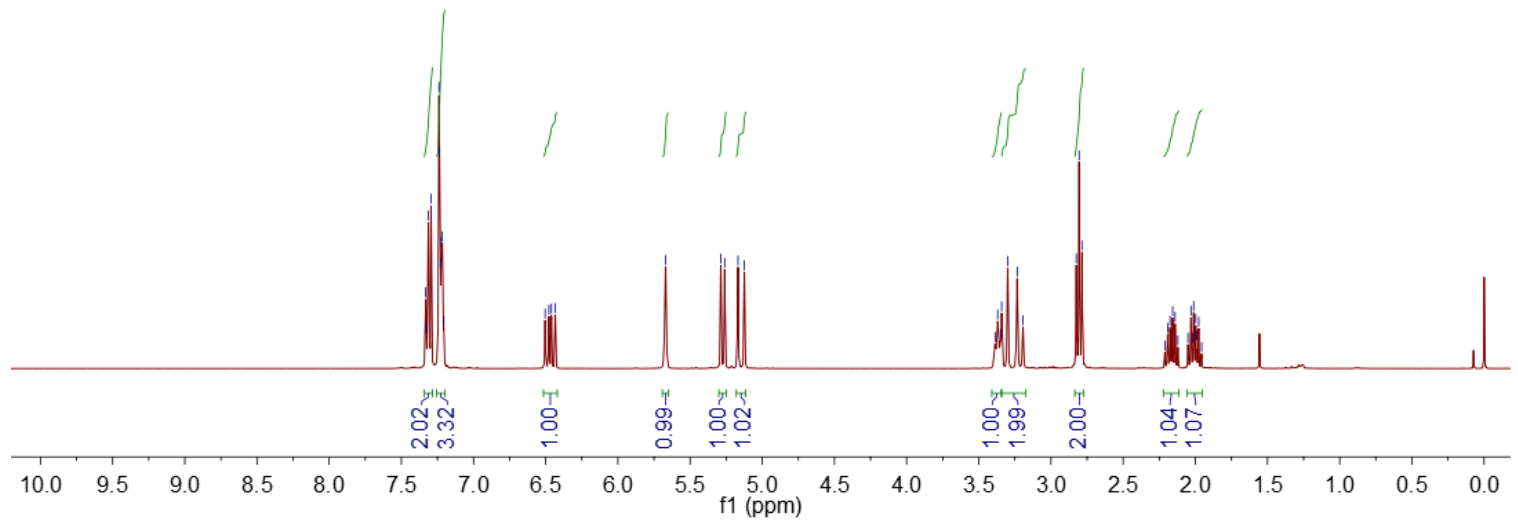

$126 \mathrm{MHz}, \mathrm{CDCl}_{3}$

[6]

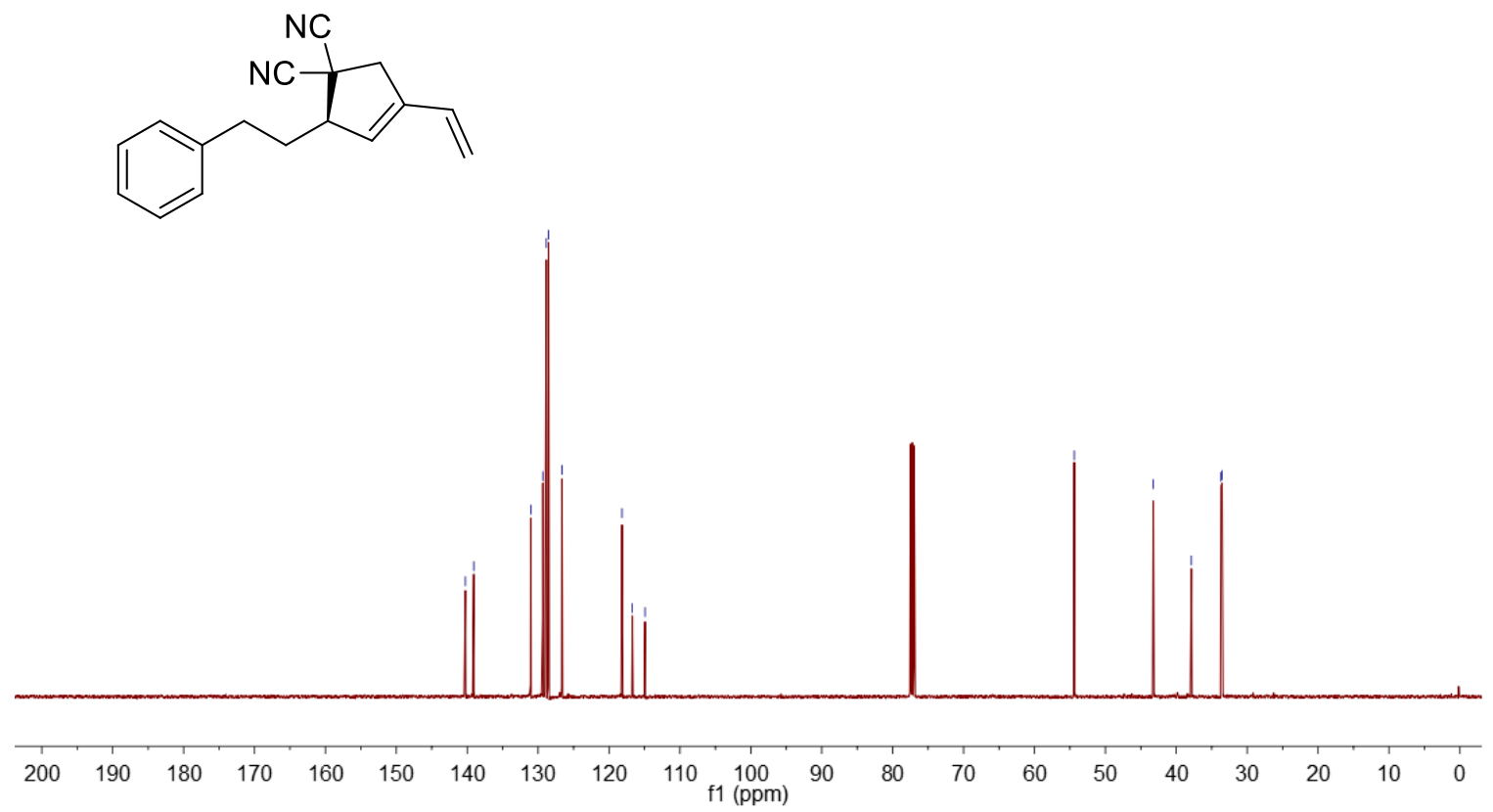


$400 \mathrm{MHz}, \mathrm{CD}_{3} \mathrm{OD}$

[7]

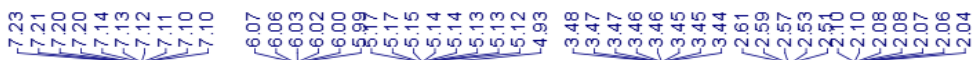<smiles>C=C[C@@H](CCc1ccccc1)c1c(N)nc(N)nc1N</smiles>

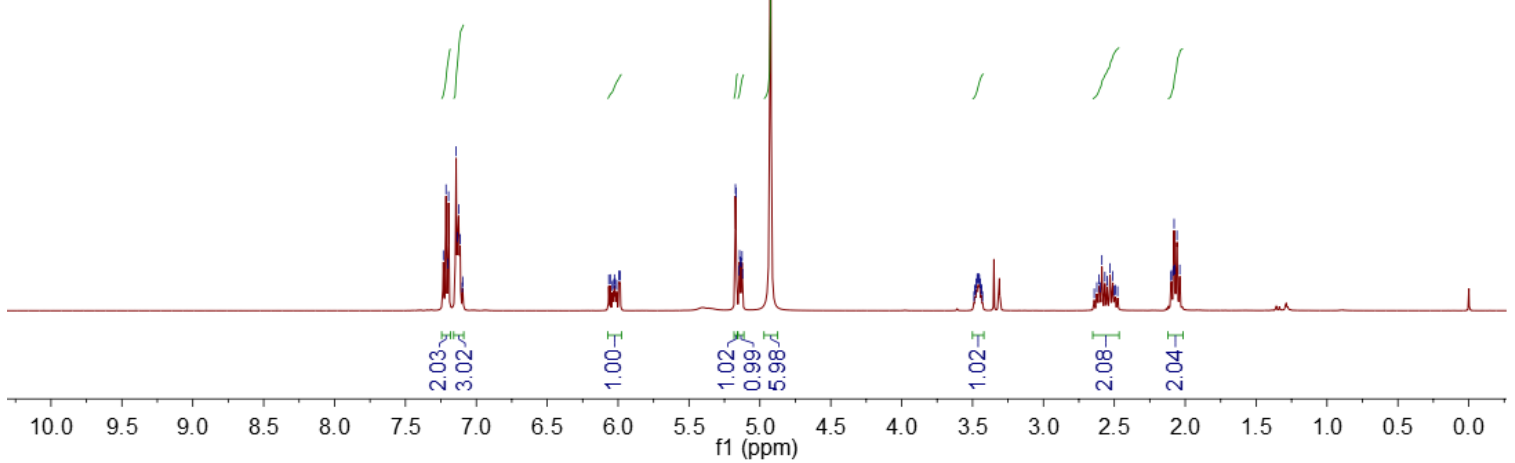

$126 \mathrm{MHz}, \mathrm{CD}_{3} \mathrm{OD}$

[7]

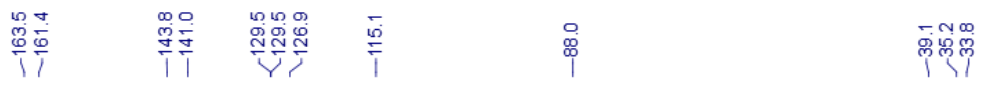<smiles>C=C[C@@H](CCc1ccccc1)c1c(N)nc(N)nc1N</smiles>

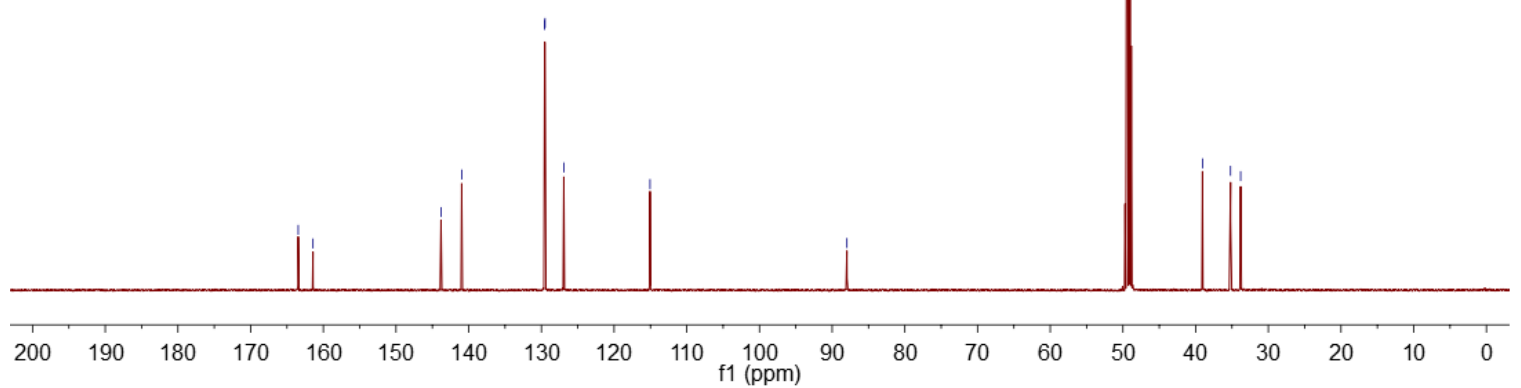


15. HPLC spectra of new compounds:

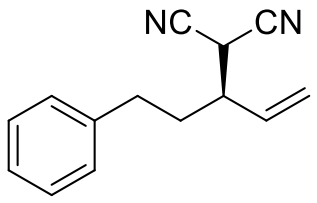

[3aa]

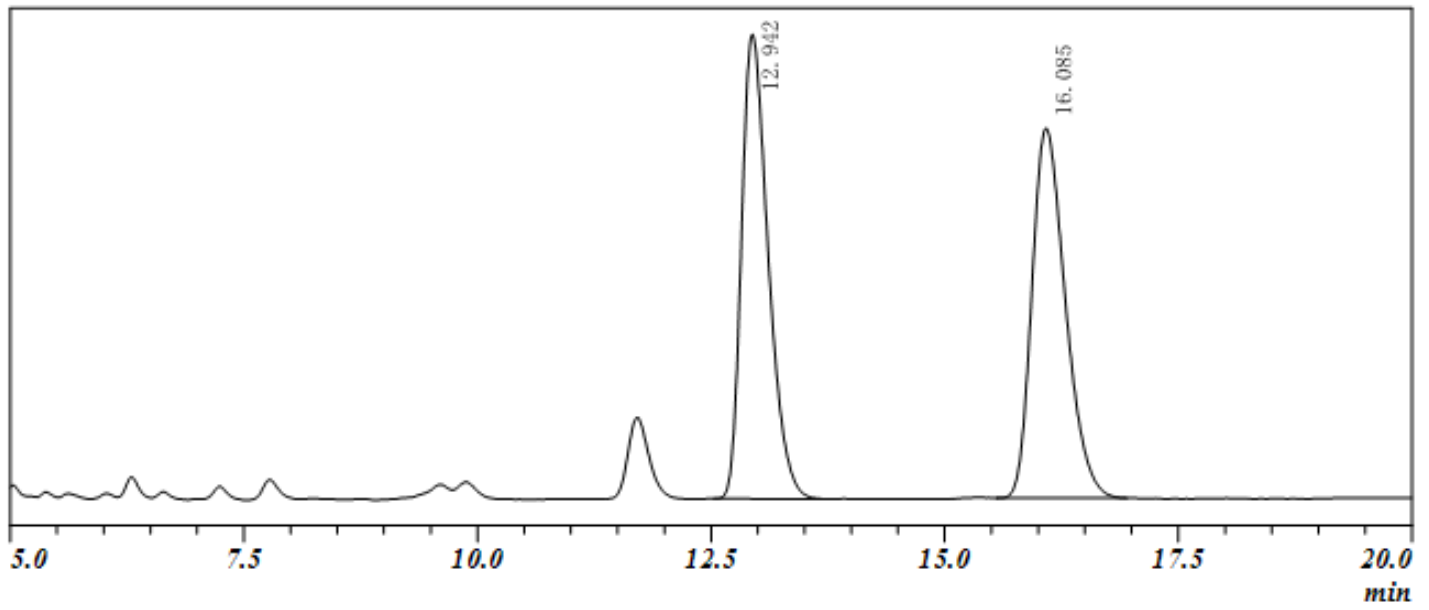

PEAK TABLE

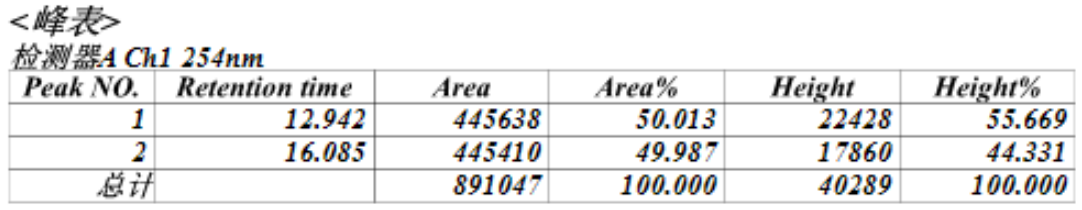

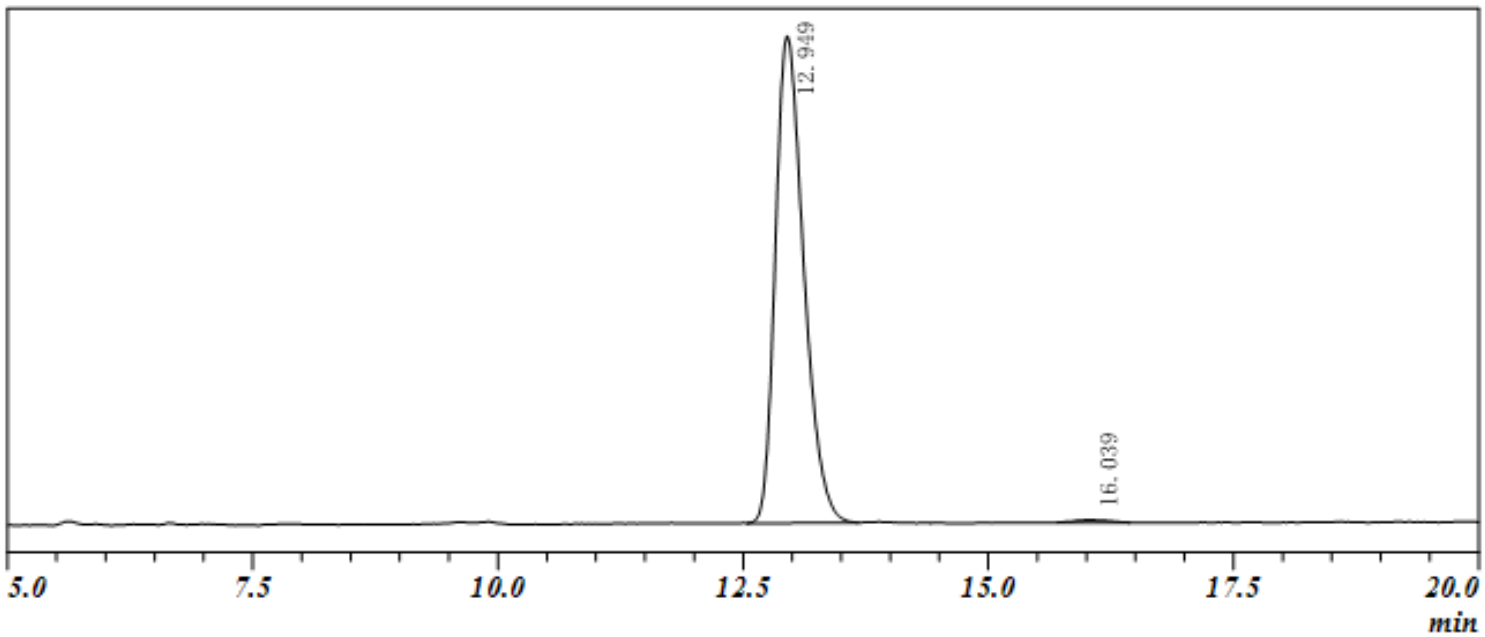

PEAK TABLE

<峰表>

检旸照A Ch1 $254 \mathrm{~nm}$

\begin{tabular}{|r|r|r|r|r|r|}
\hline Peak NO. & Retention time & \multicolumn{1}{c|}{ Area } & \multicolumn{1}{c|}{ Area\% } & \multicolumn{1}{c|}{ Height } & \multicolumn{1}{c|}{ Height $\%$} \\
\hline 1 & 12.949 & 372283 & 99.406 & 18484 & 99.485 \\
\hline 2 & 16.039 & 2226 & 0.594 & 96 & 0.515 \\
\hline 总计 & & 374509 & 100.000 & 18579 & 100.000 \\
\hline
\end{tabular}




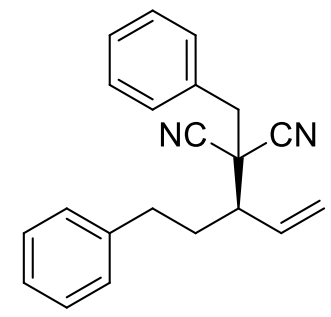

[3ab]

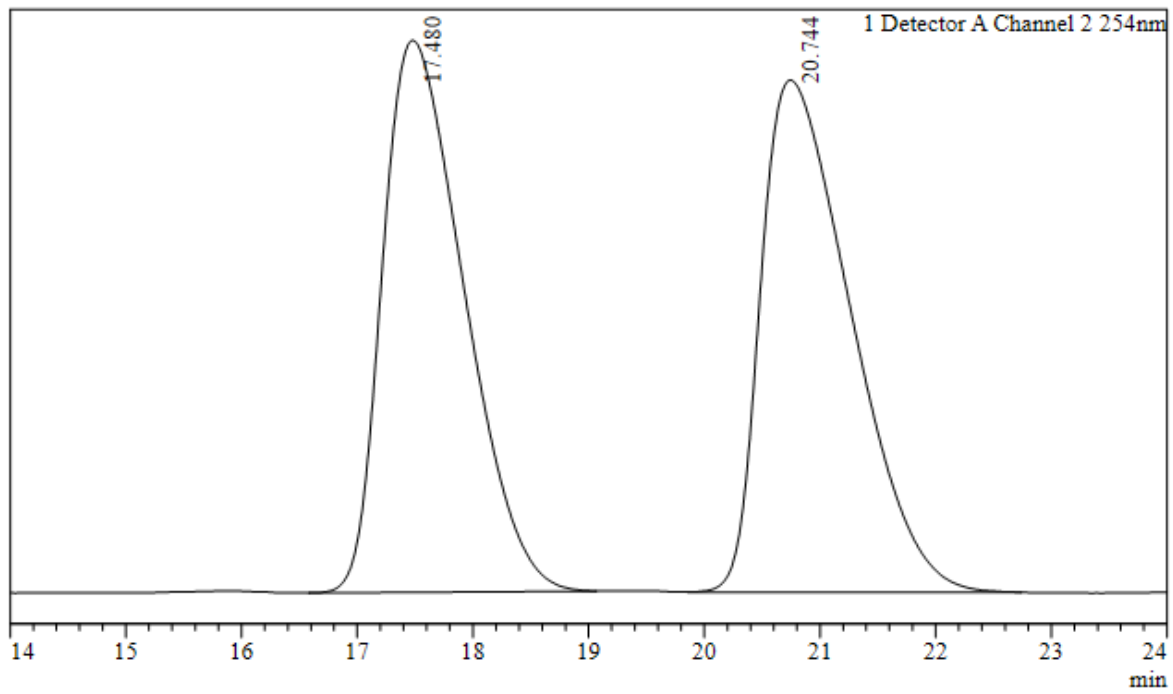

Peak Table

\begin{tabular}{|c|c|c|c|c|c|}
\hline tector $A$ & Chan & & & & \\
\hline${ }_{1}$ & Ret. Time & $\begin{array}{l}\text { Area } \\
2565596\end{array}$ & Area $\%$ & Height & Height $\%$ \\
\hline 2 & 20.744 & 26265 & 50.587 & 48559 & 48.124 \\
\hline Total & & 5192120 & 100.000 & 100903 & 100.000 \\
\hline
\end{tabular}

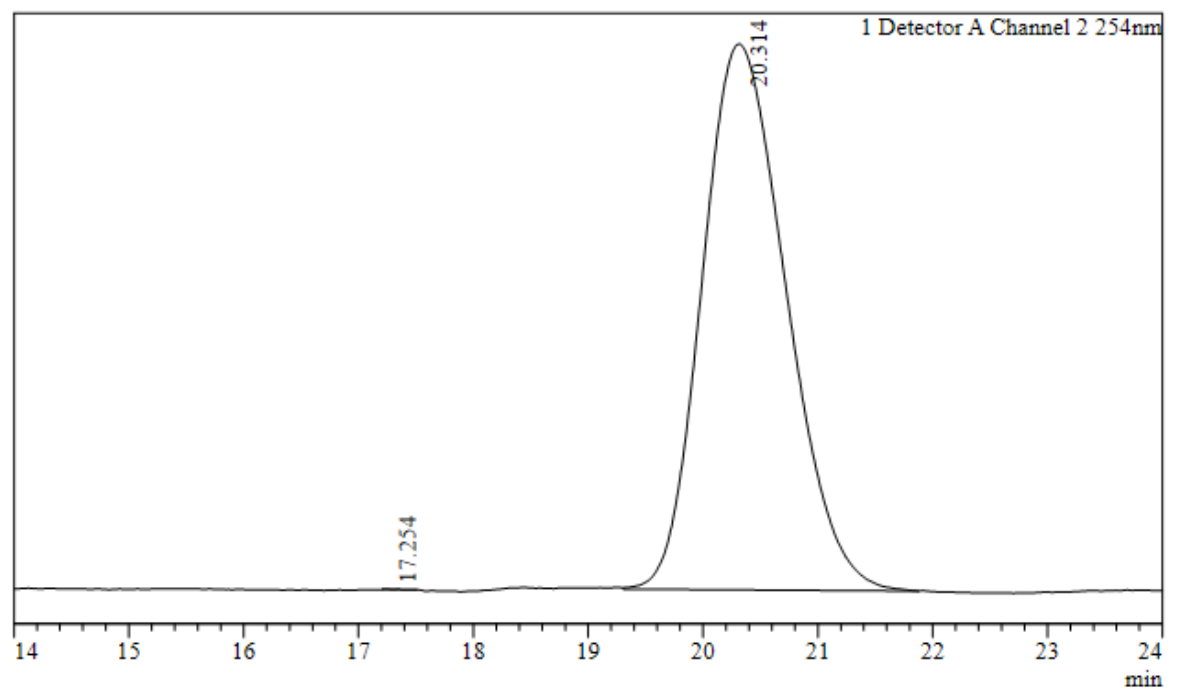

Peak Table

\begin{tabular}{|c|c|c|c|c|c|}
\hline $\begin{array}{r}\text { Peak\# } \\
1\end{array}$ & $\begin{array}{r}\text { Ret. Time } \\
17.254\end{array}$ & Area & $\begin{array}{l}\text { Area } \% \\
0.001\end{array}$ & $\begin{array}{ll}\text { Height } & \\
& \end{array}$ & $\begin{array}{c}\text { Height } \% \\
0.016\end{array}$ \\
\hline 2 & 20.314 & 704197 & 99.999 & 13856 & 99.984 \\
\hline Total & & 704206 & 100.000 & 13858 & 100.000 \\
\hline
\end{tabular}




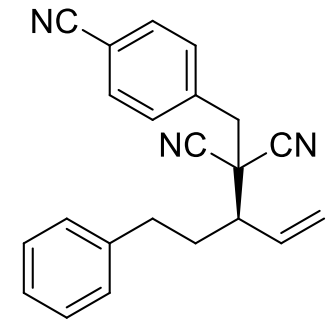

[3ac]

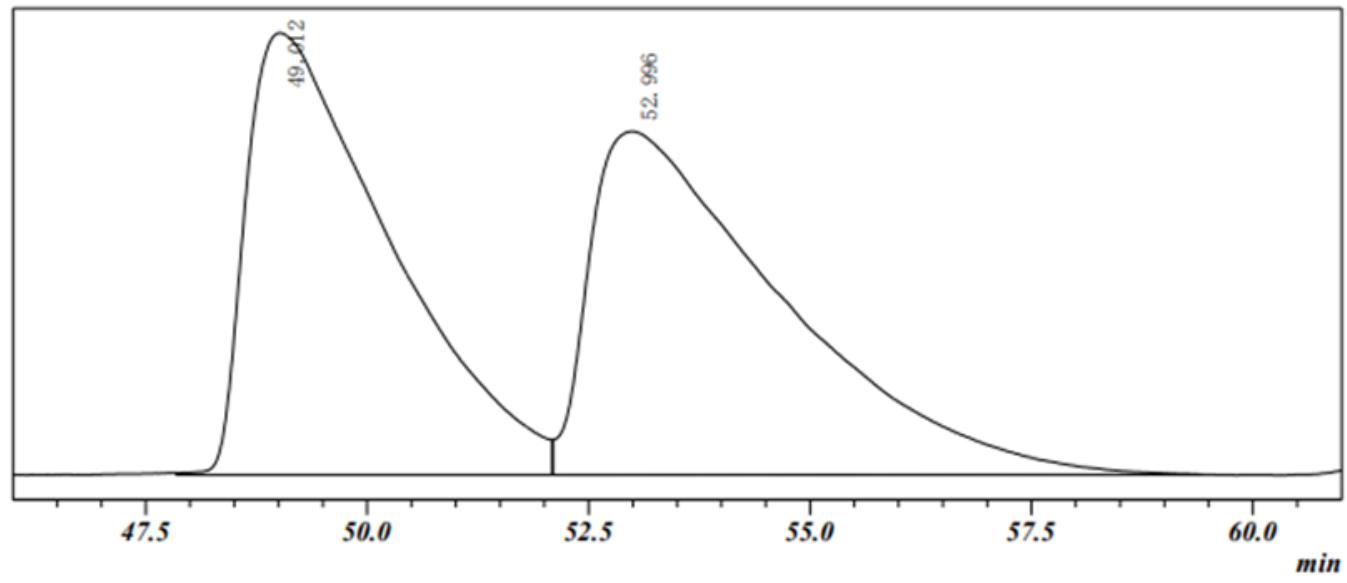

PEAK TABLE
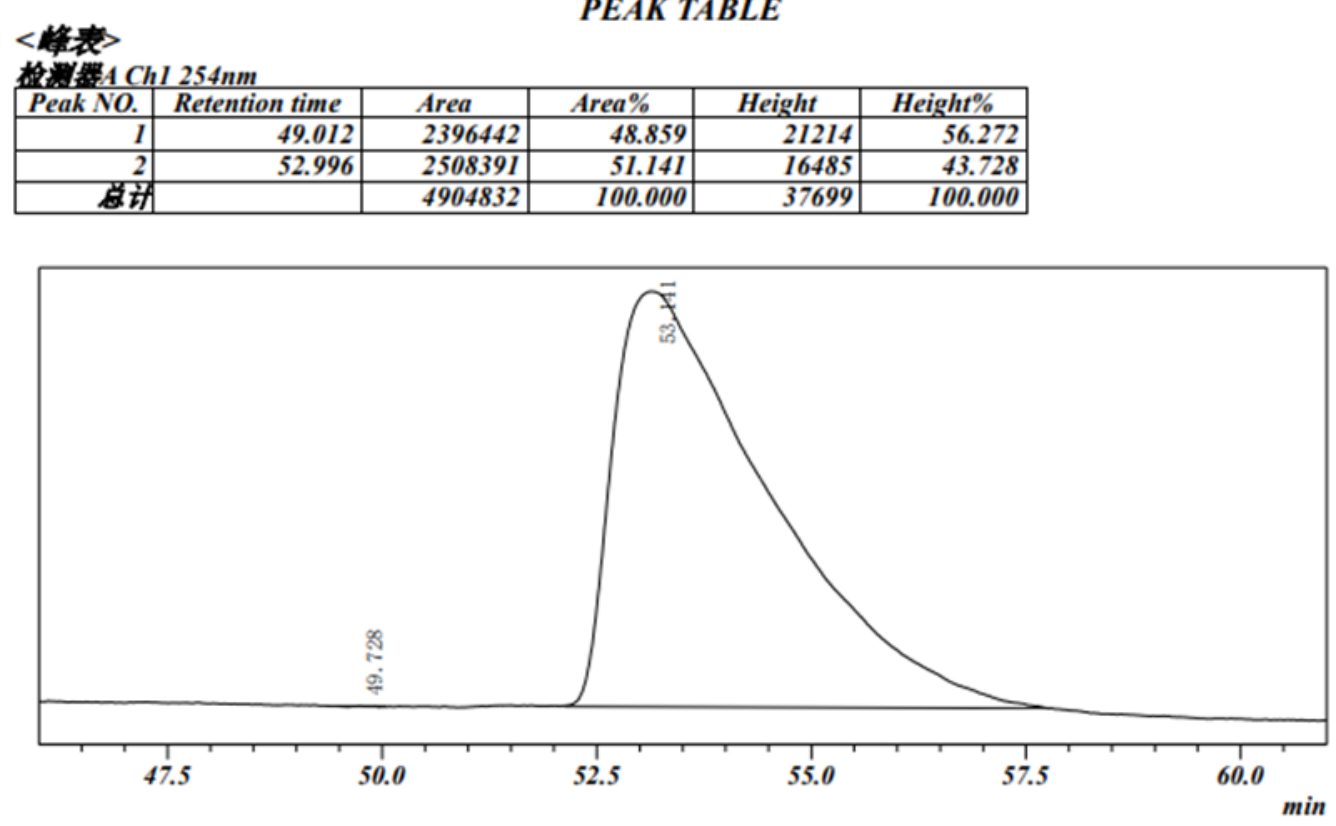

PEAK TABLE

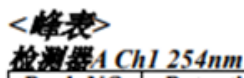

\begin{tabular}{|c|c|c|c|c|c|}
\hline Peak NO. & Retention time & Area & Area\% & Height & Height $\%$ \\
\hline 1 & 49.728 & 16 & 0.001 & 14 & 0.133 \\
\hline 2 & 53.141 & 1368344 & 99.999 & 10787 & 99.867 \\
\hline 总咞 & & 1368360 & 100.000 & 10801 & 100.000 \\
\hline
\end{tabular}




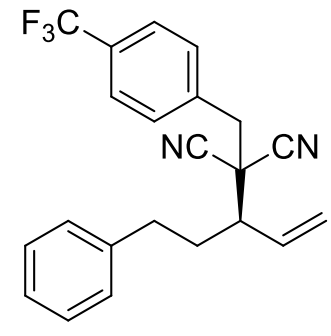

[3ad]

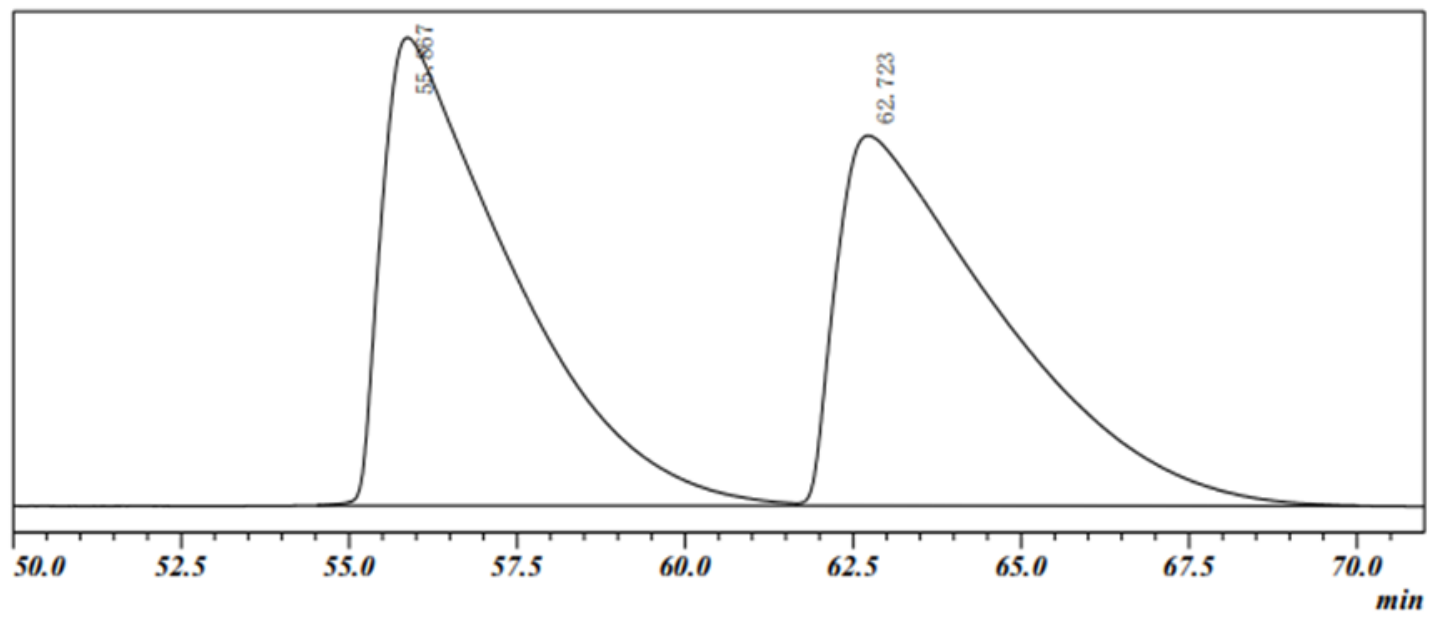

PEAK TABLE

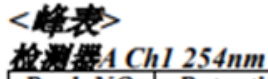

\begin{tabular}{|r|r|r|r|r|r|}
\hline Peak NO. & Retention time & \multicolumn{1}{|c|}{ Area } & \multicolumn{1}{|c|}{ Area \% } & \multicolumn{1}{c|}{ Height } & \multicolumn{1}{c|}{ Height $\%$} \\
\hline 1 & 55.867 & 5838927 & 50.055 & 43380 & 55.836 \\
\hline 2 & 62.723 & 5826080 & 49.945 & 34311 & 44.164 \\
\hline 尊计 & & 11665007 & 100.000 & 77692 & 100.000 \\
\hline
\end{tabular}

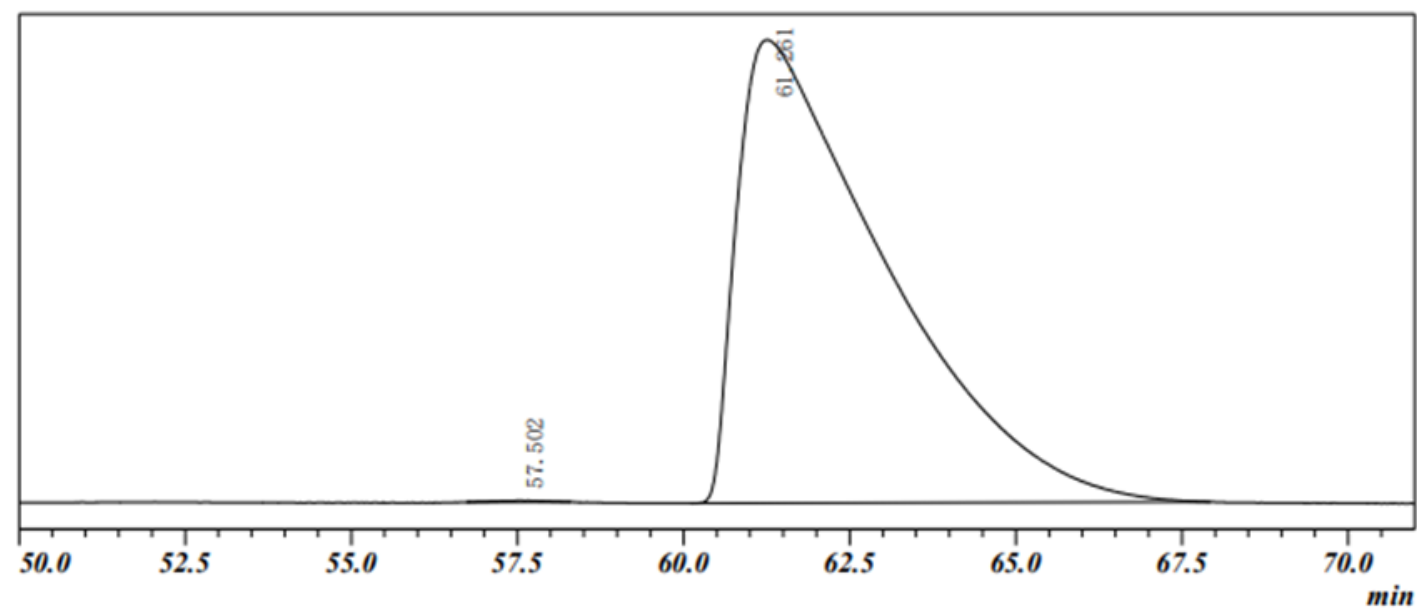

<峰表>

PEAK TABLE

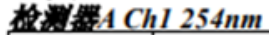

\begin{tabular}{|r|r|r|r|r|r|}
\hline Peak NO. & Retention time & \multicolumn{1}{c|}{ Area } & \multicolumn{1}{c|}{ Area\% } & \multicolumn{1}{c|}{ Height } & \multicolumn{1}{|c|}{ Height $\%$} \\
\hline 1 & 57.502 & 5098 & 0.103 & 103 & 0.325 \\
\hline 2 & 61.261 & 4951409 & 99.897 & 31776 & 99.675 \\
\hline 计 & & 4956507 & 100.000 & 31880 & 100.000 \\
\hline
\end{tabular}




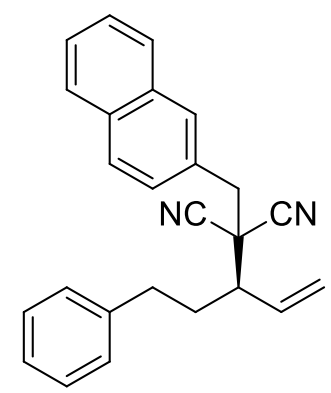

[3ae]

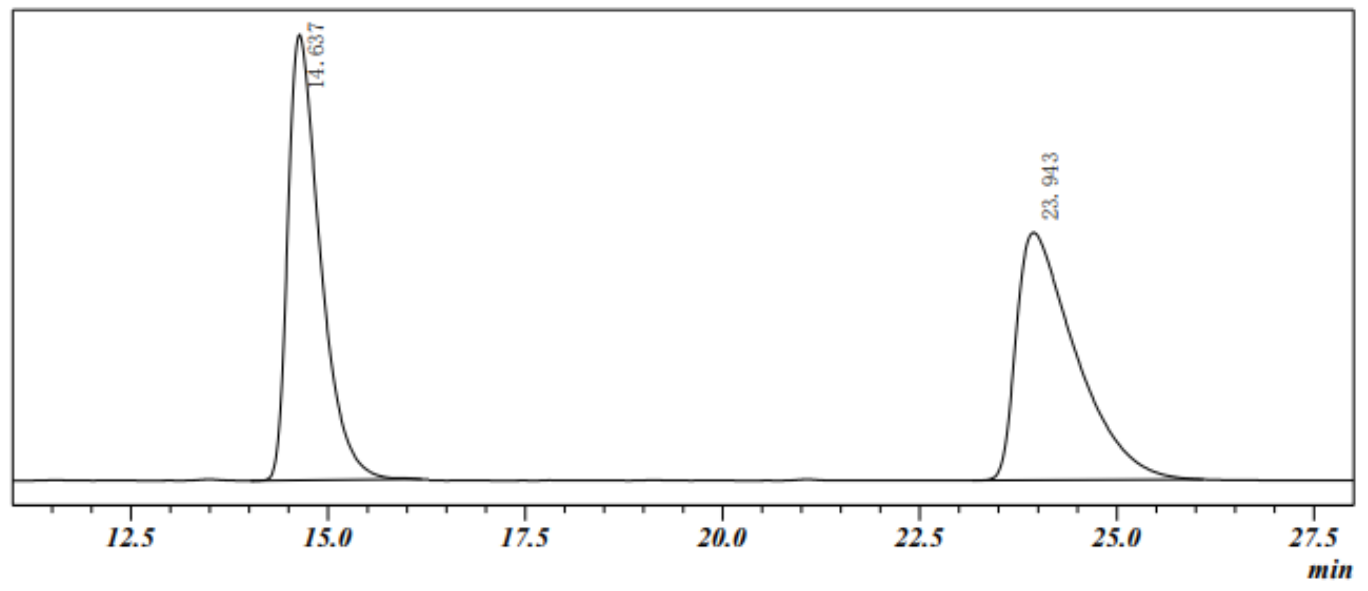

PEAK TABLE

<峰表

枌清罢A ChI $254 \mathrm{~nm}$

\begin{tabular}{|c|c|c|c|c|c|}
\hline Peak NO. & Retention time & Area & Area $\%$ & Height & Height $\%$ \\
\hline$I$ & 14.637 & 7714101 & 49.580 & 269138 & 64.280 \\
\hline 2 & 23.943 & 7844726 & 50.420 & 149557 & 35.720 \\
\hline 意计 & & 15558827 & 100.000 & 418695 & 100.000 \\
\hline
\end{tabular}

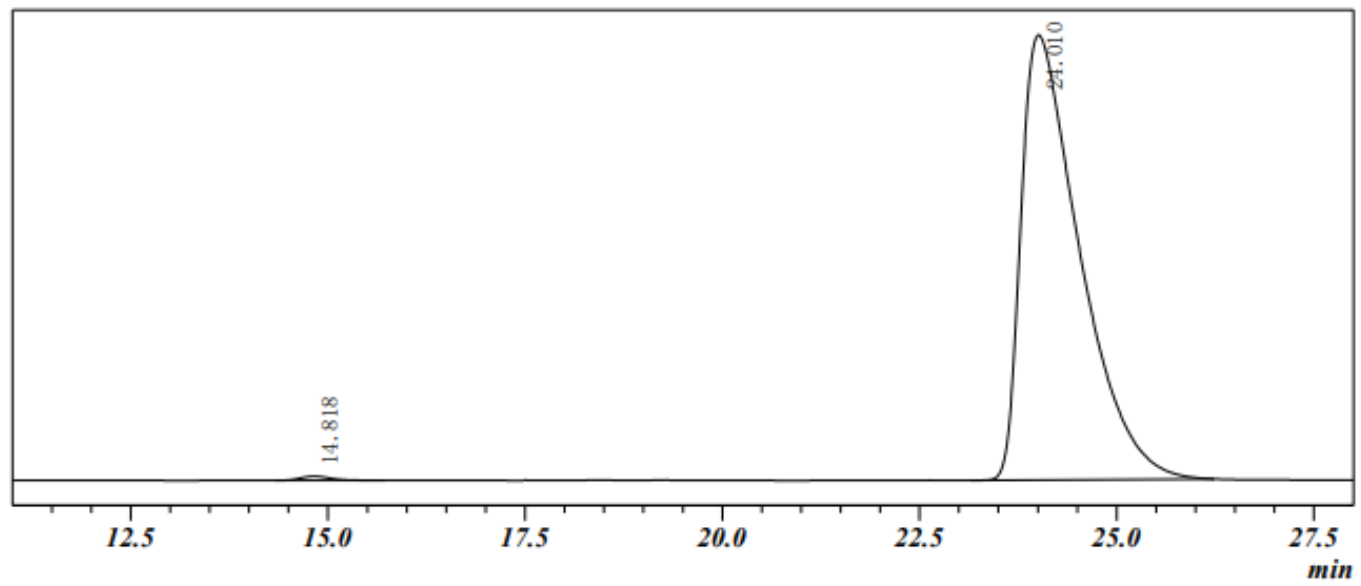

PEAK TABLE

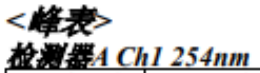

\begin{tabular}{|r|r|r|r|r|r|}
\hline Peak NO. & Retention time & \multicolumn{1}{c|}{ Area } & \multicolumn{1}{c|}{ Area\% } & \multicolumn{1}{c|}{ Height } & \multicolumn{1}{c|}{ Height $\%$} \\
\hline 1 & 14.818 & 32896 & 0.494 & 1214 & 0.939 \\
\hline 2 & 24.010 & 6620326 & 99.506 & 128047 & 99.061 \\
\hline 总计 & & 6653222 & 100.000 & 129261 & 100.000 \\
\hline
\end{tabular}




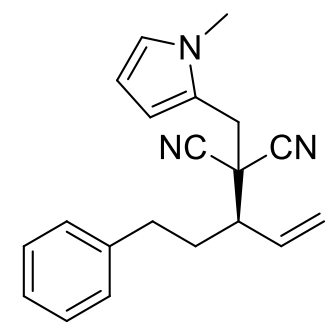

[3af]

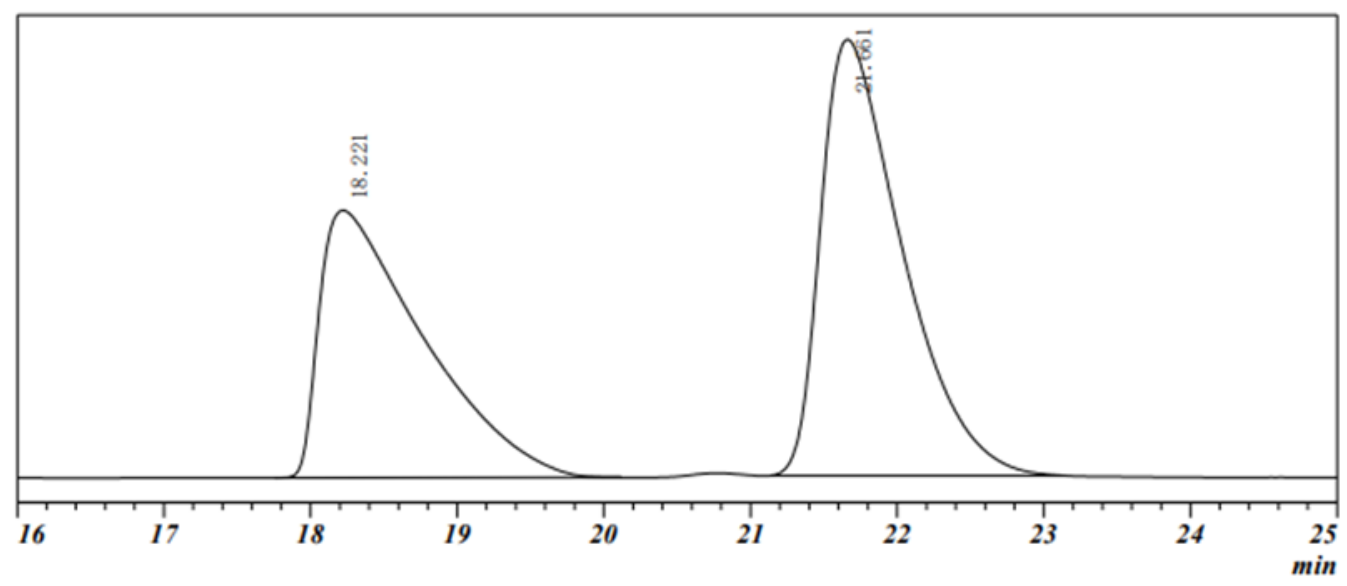

PEAK TABLE

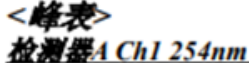

\begin{tabular}{|l|c|c|c|c|c|}
\hline Peak NO. & Retention time & Area & Area $\%$ & Height & Height $\%$ \\
\hline
\end{tabular}

\begin{tabular}{|r|r|r|r|r|r|}
\hline Peak NO. & Retention time & \multicolumn{1}{|c|}{ Area } & \multicolumn{1}{c|}{ Area $\%$} & \multicolumn{1}{c|}{ Height } & \multicolumn{1}{c|}{ Height $\%$} \\
\hline$I$ & 18.221 & 1914980 & 43.783 & 38940 & 38.009 \\
\hline 2 & 21.661 & 2458813 & 56.217 & 63509 & 61.991 \\
\hline 放 & & 4373793 & 100.000 & 102449 & 100.000 \\
\hline
\end{tabular}

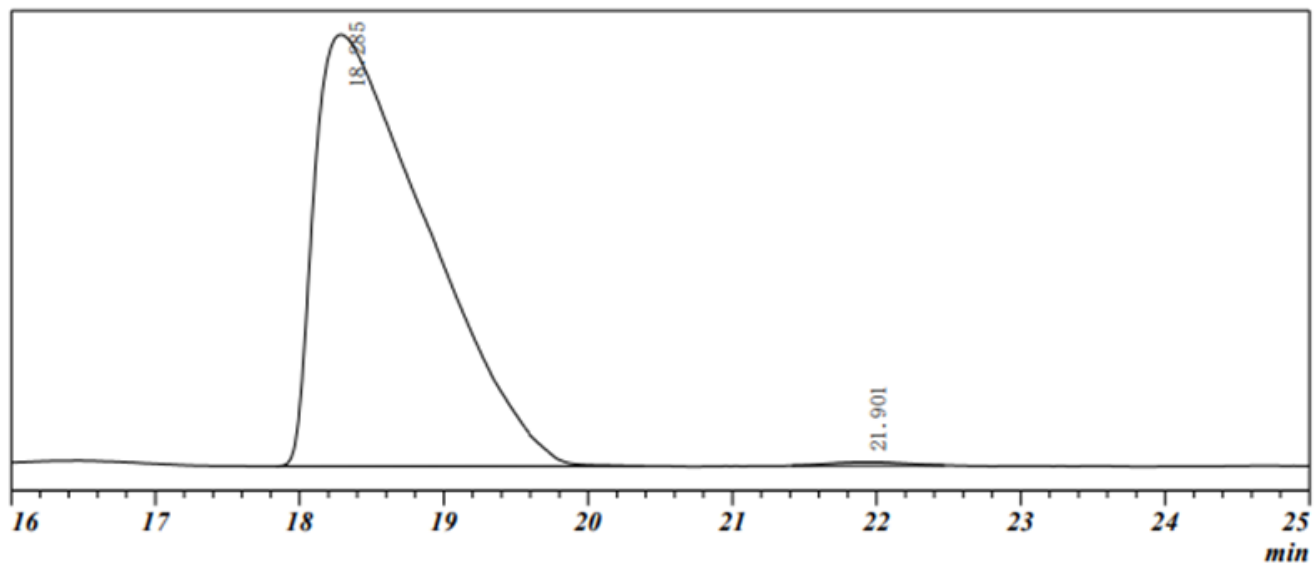

PEAK TABLE

\section{<蝼表}

\begin{tabular}{|r|r|r|r|r|r|}
\hline Peak NO. & Retention time & \multicolumn{1}{|c|}{ Area } & \multicolumn{1}{|c|}{ Area\% } & \multicolumn{1}{c|}{ Height } & \multicolumn{1}{|c|}{ Height\% } \\
\hline$I$ & 18.285 & 2118634 & 99.512 & 37319 & 99.202 \\
\hline 2 & 21.901 & 10393 & 0.488 & 300 & 0.798 \\
\hline 尊计 & & 2129027 & 100.000 & 37619 & 100.000 \\
\hline
\end{tabular}




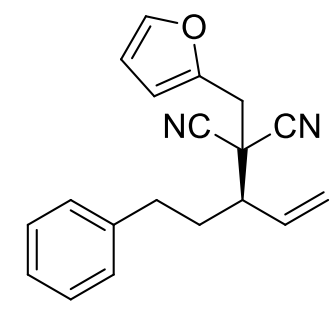

[3ag]

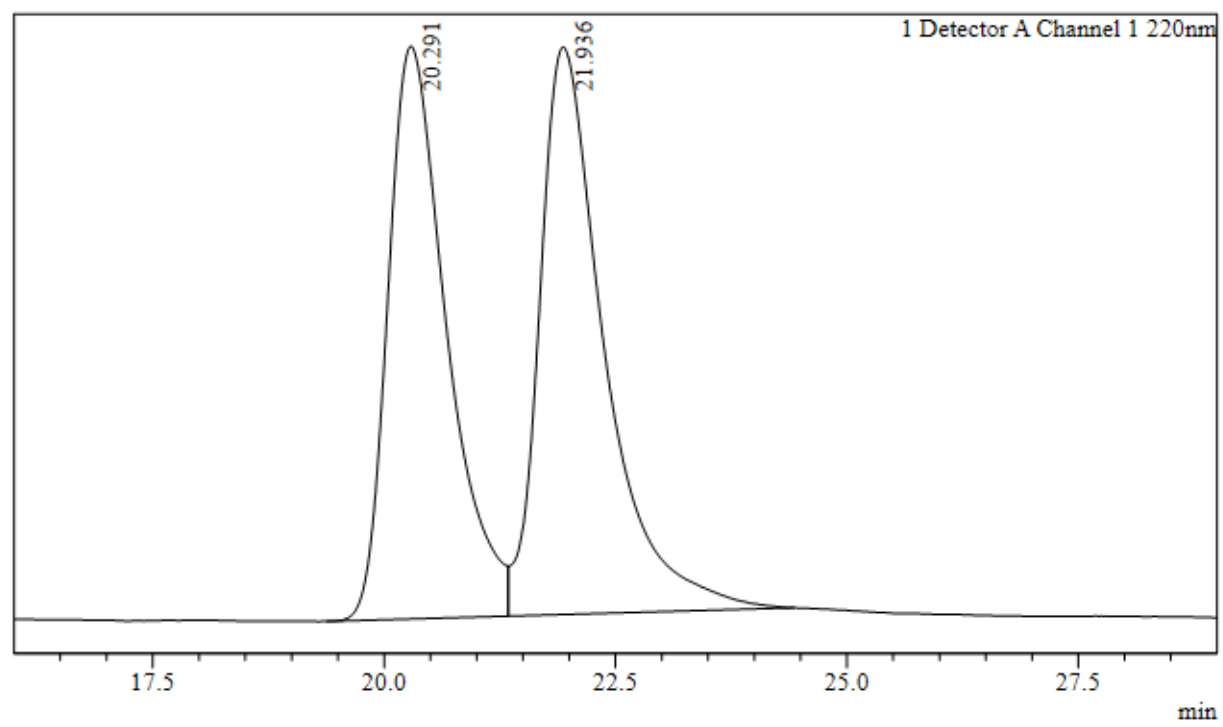

Peak Table
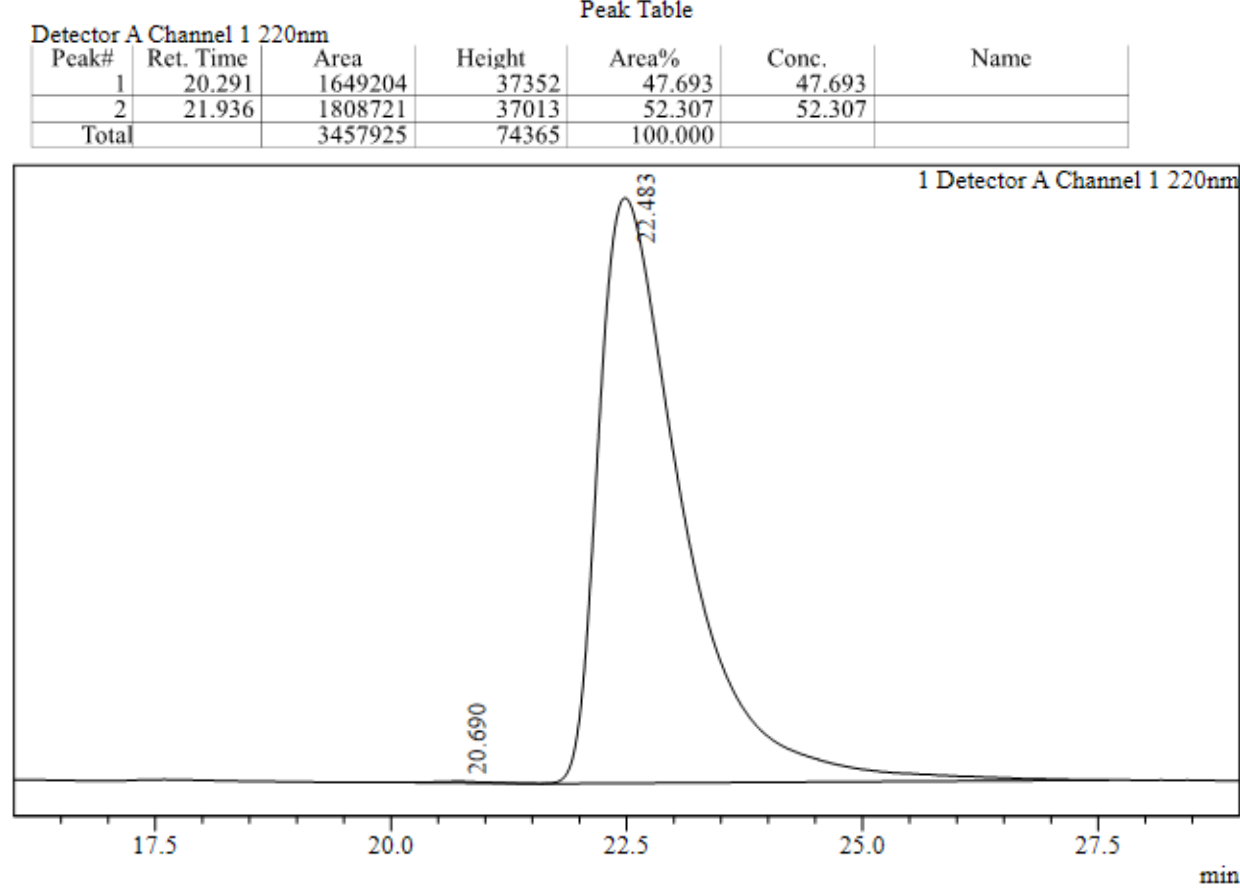

Peak Table

\begin{tabular}{|c|c|c|c|c|c|c|}
\hline \multicolumn{7}{|c|}{ Detector A Channel $1220 \mathrm{~nm}$} \\
\hline Peak\# & Ret. Time & Area & Height ${ }_{167}$ & Area $\%$ & Conc. & Name \\
\hline 2 & 22.483 & 4871944 & 77709 & 99.868 & 99.868 & \\
\hline Tota & & 4878368 & 77875 & 100.000 & & \\
\hline
\end{tabular}




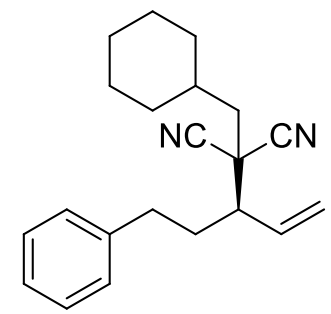

[3ah]

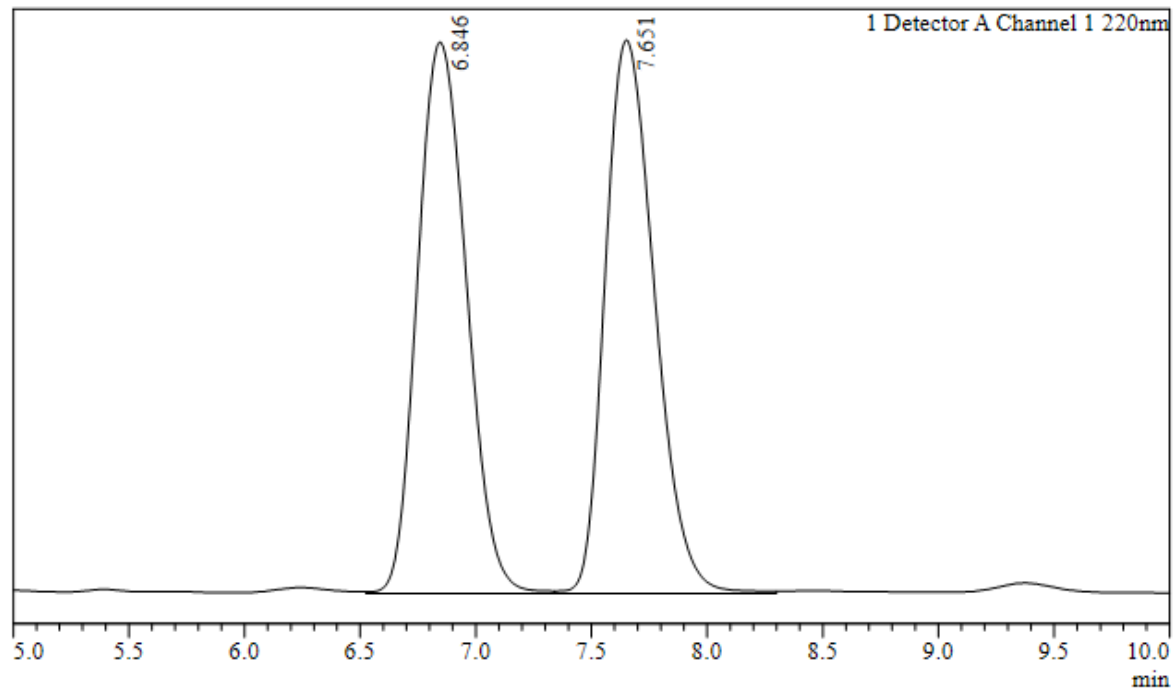

Peak Table

\begin{tabular}{|c|c|c|c|c|c|c|}
\hline Detector & A Channel 1 & $\mathrm{~nm}$ & & & & \\
\hline $\begin{array}{r}\text { Peak\# }_{1} \\
\end{array}$ & $\begin{array}{r}\text { Ret. Time } \\
6.846\end{array}$ & $\begin{array}{l}\text { Area } \\
1293880\end{array}$ & $\begin{array}{l}\text { Height } \\
87549\end{array}$ & $\begin{array}{l}\text { Area } \% \\
49.678\end{array}$ & $\begin{array}{l}\text { Conc. } \\
49.678\end{array}$ & Name \\
\hline 2 & 7.651 & 1310653 & 87951 & 50.322 & 50.322 & \\
\hline Tota & & 2604532 & 175500 & 100.000 & & \\
\hline
\end{tabular}

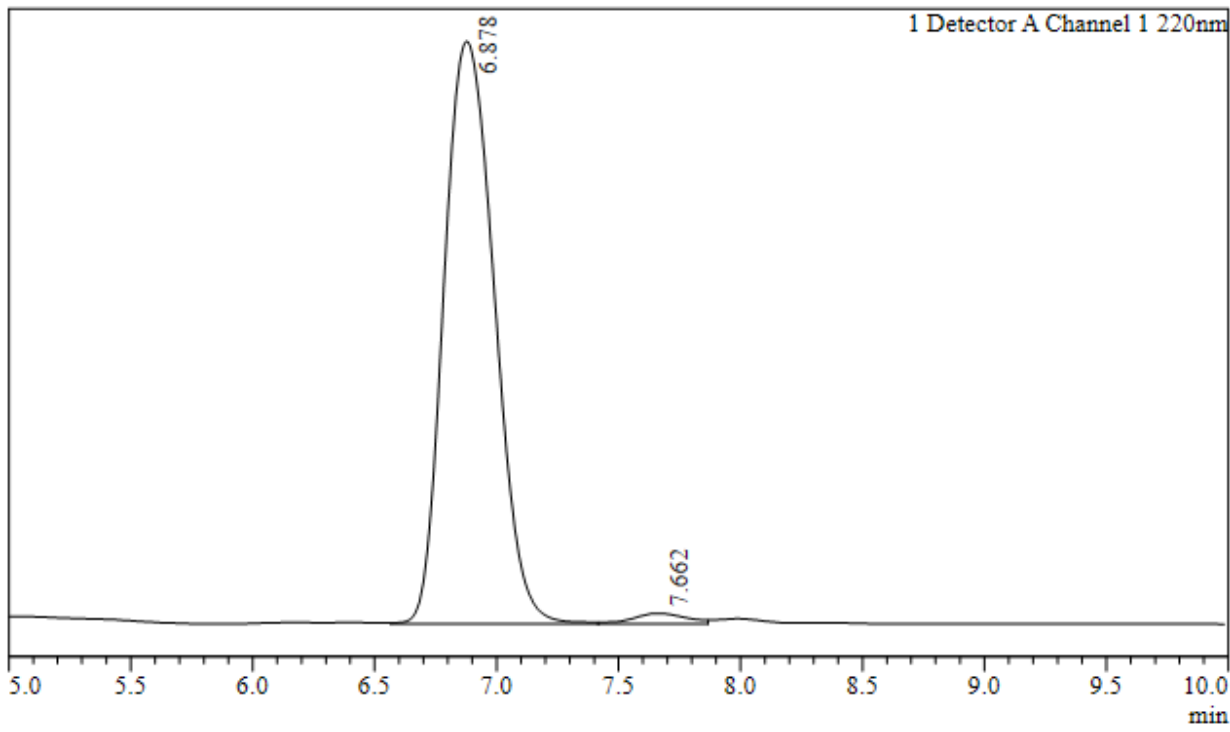

Peak Table

\begin{tabular}{|c|c|c|c|c|c|c|}
\hline Detector $A$ & Channel 1 & & & & & \\
\hline Peak\# $_{1}$ & $\begin{array}{r}\text { Ret. Time } \\
6.878\end{array}$ & $\begin{array}{l}\text { Area } \\
3194491\end{array}$ & $\begin{array}{l}\text { Height } \\
222366\end{array}$ & $\begin{array}{l}\text { Area } \% \\
98.067\end{array}$ & $\begin{array}{l}\text { Conc. } \\
98.067\end{array}$ & Name \\
\hline 2 & 7.662 & 62980 & 4035 & 1.933 & 1.933 & \\
\hline Total & & 3257471 & 226401 & 100.000 & & \\
\hline
\end{tabular}


<smiles>C=CCC(C#N)(C#N)C(C=C)CCc1ccccc1</smiles>

[3ai]

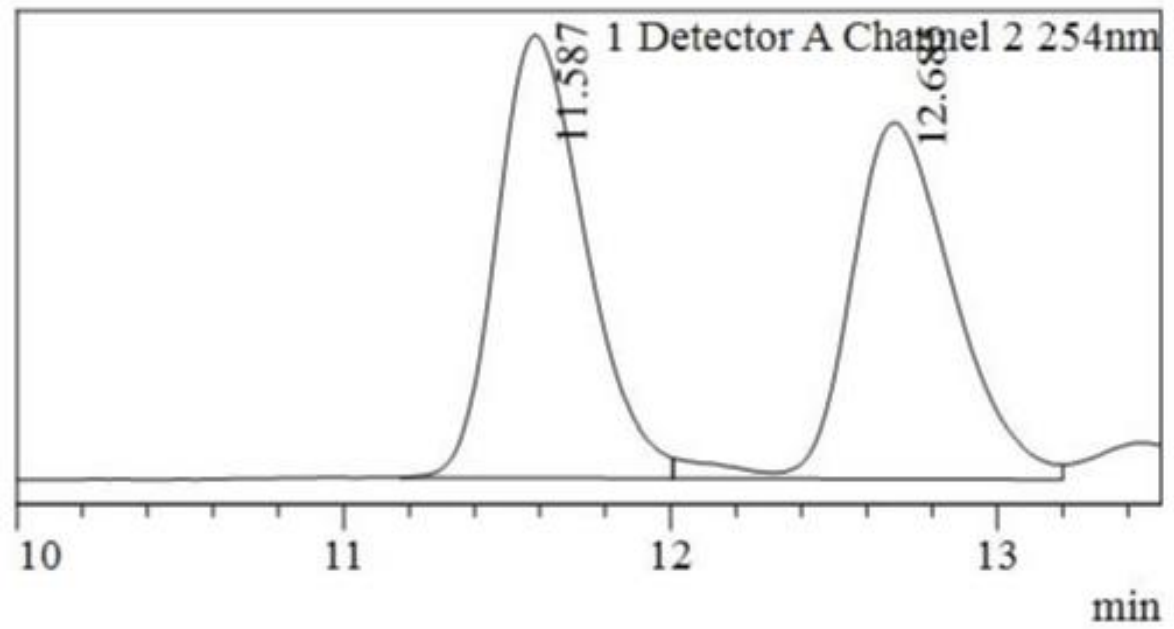

\begin{tabular}{|c|c|c|c|c|c|c|}
\hline \multicolumn{7}{|c|}{ Detector A Channel 2 254nm } \\
\hline Peak\# & Ret. Time & Area & ${ }_{7246}^{\text {Height }}$ & Area $\%$ & Conc. & Name \\
\hline 2 & 12.685 & 129171 & 5833 & 48.544 & 48.544 & \\
\hline Total & & 266089 & 13080 & 100.000 & & \\
\hline
\end{tabular}

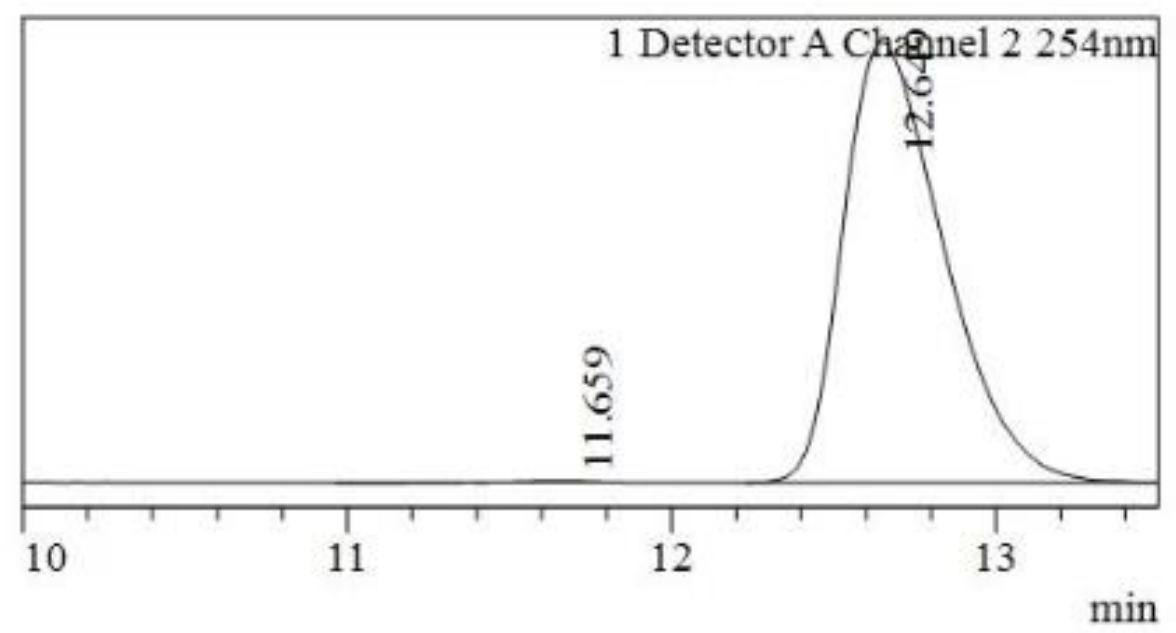

\begin{tabular}{|c|c|c|c|c|c|c|}
\hline \multicolumn{7}{|c|}{ Detector A Channel 2} \\
\hline Peak\# & $\begin{array}{r}\text { Ret. Time } \\
11.659\end{array}$ & ${ }_{255}$ & Height & $\begin{array}{l}\text { Area } \% \\
0.117\end{array}$ & $\begin{array}{l}\text { Conc. } \\
0.117\end{array}$ & Name \\
\hline 2 & 12.649 & 216522 & 9986 & 99.883 & 99.883 & \\
\hline Total & & 216777 & 10018 & 100.000 & & \\
\hline
\end{tabular}




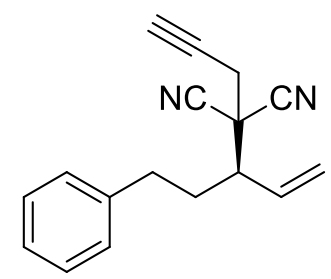

[3aj]

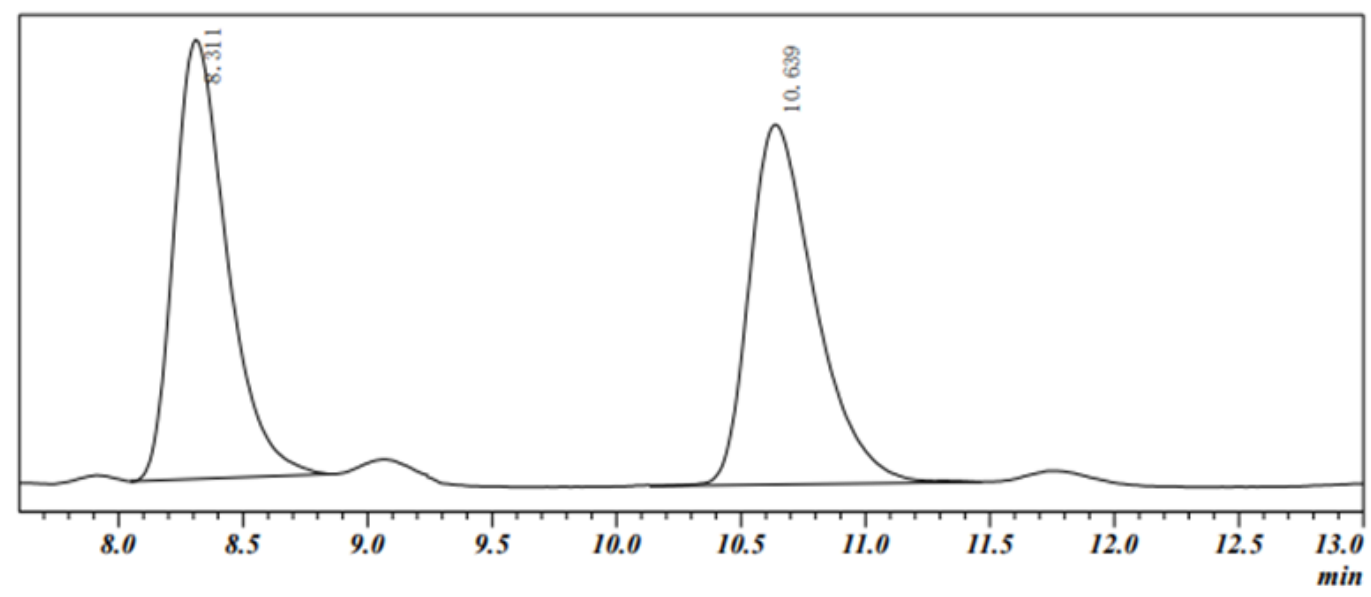

PEAK TABLE

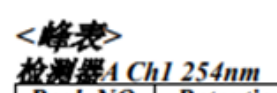

\begin{tabular}{|r|r|r|r|r|r|}
\hline Peak NO. & Retention time & \multicolumn{1}{c|}{ Area } & \multicolumn{1}{c|}{ Area\% } & \multicolumn{1}{c|}{ Height } & \multicolumn{1}{|c|}{ Height $\%$} \\
\hline$I$ & 8.311 & 204038 & 49.592 & 13778 & 54.983 \\
\hline 2 & 10.639 & 207392 & 50.408 & 11281 & 45.017 \\
\hline 兽计 & & 411429 & 100.000 & 25058 & 100.000 \\
\hline
\end{tabular}

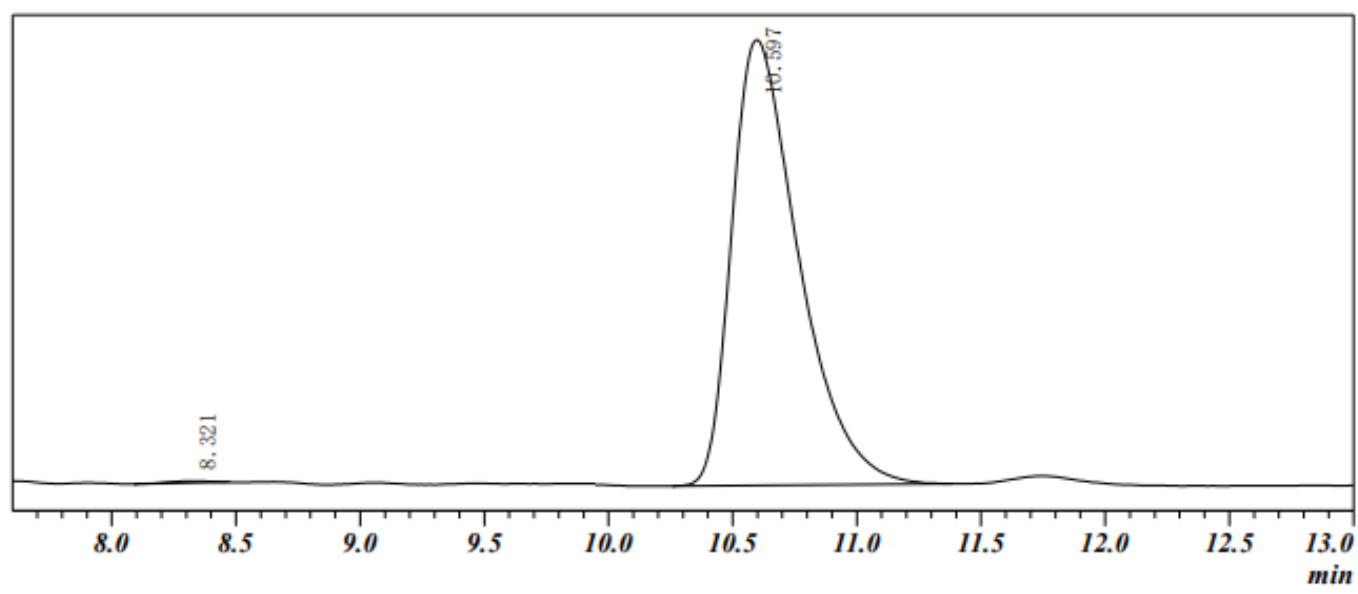

PEAK TABLE

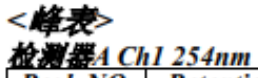

\begin{tabular}{|r|r|r|r|r|r|}
\hline Peak NO. & Retention time & \multicolumn{1}{c|}{ Area } & \multicolumn{1}{c|}{ Area\% } & \multicolumn{1}{c|}{ Height } & \multicolumn{1}{c|}{ Height $\%$} \\
\hline 1 & 8.321 & 1004 & 0.271 & 87 & 0.443 \\
\hline 2 & 10.597 & 368719 & 99.729 & 19634 & 99.557 \\
\hline 总计 & & 369723 & 100.000 & 19721 & 100.000 \\
\hline
\end{tabular}




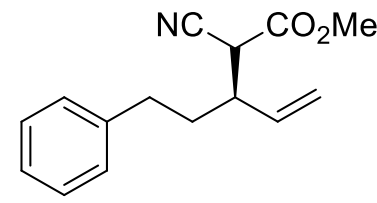

[3ak]

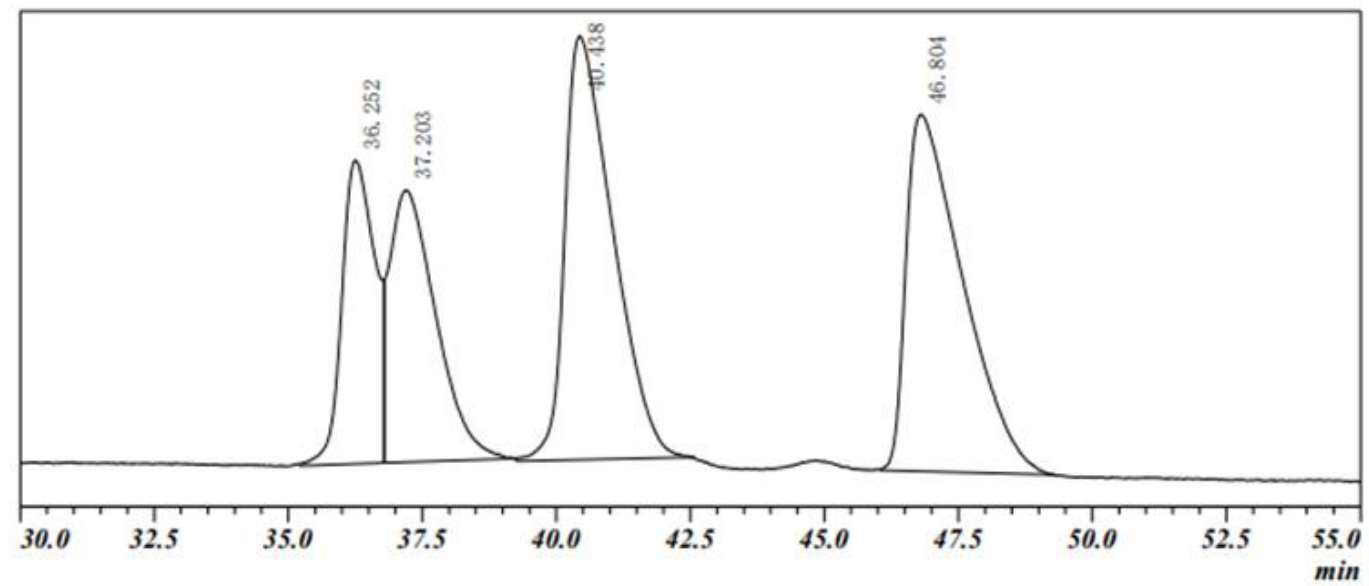

PEAK TABLE

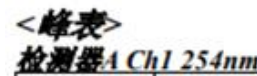

\begin{tabular}{|r|r|r|r|r|r|}
\hline Peak NO. & Retention time & \multicolumn{1}{c|}{ Area } & \multicolumn{1}{c|}{ Area\% } & \multicolumn{1}{c|}{ Height } & \multicolumn{1}{c|}{ Height \% } \\
\hline 1 & 36.252 & 250839 & 16.021 & 5796 & 22.400 \\
\hline 2 & 37.203 & 310104 & 19.806 & 5189 & 20.054 \\
\hline 3 & 40.438 & 502717 & 32.108 & 8086 & 31.248 \\
\hline 4 & 46.804 & 502063 & 32.066 & 6805 & 26.298 \\
\hline 计 & & 1565723 & 100.000 & 25876 & 100.000 \\
\hline
\end{tabular}

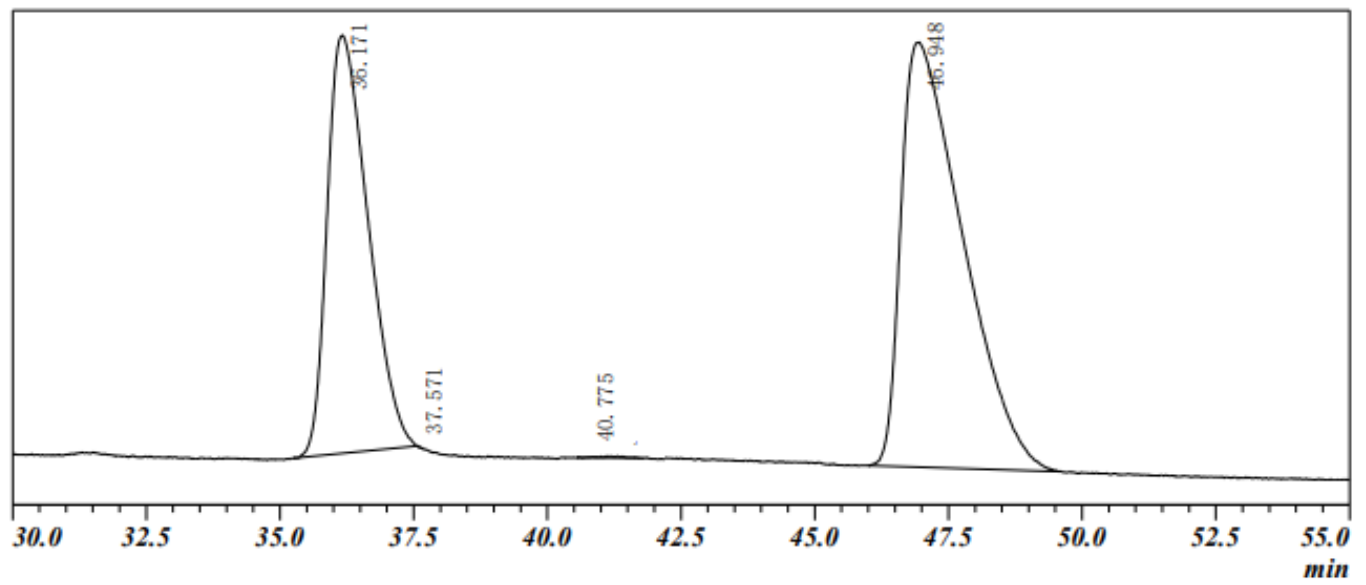

PEAK TABLE

<峰衣

\begin{tabular}{|r|r|r|r|r|r|}
\hline Peak NO. & Retention time & \multicolumn{1}{c|}{ Area } & \multicolumn{1}{c|}{ Area\% } & \multicolumn{1}{c|}{ Height } & \multicolumn{1}{c|}{ Height\% } \\
\hline 1 & 36.171 & 332437 & 39.364 & 6255 & 49.473 \\
\hline 2 & 37.571 & 2 & 0.000 & 0 & 0.001 \\
\hline 3 & 40.775 & 290 & 0.034 & 30 & 0.235 \\
\hline 4 & 46.948 & 511795 & 60.602 & 6359 & 50.290 \\
\hline 总调 & & 844525 & 100.000 & 12644 & 100.000 \\
\hline
\end{tabular}




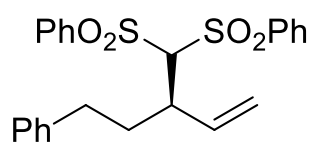

[3al]

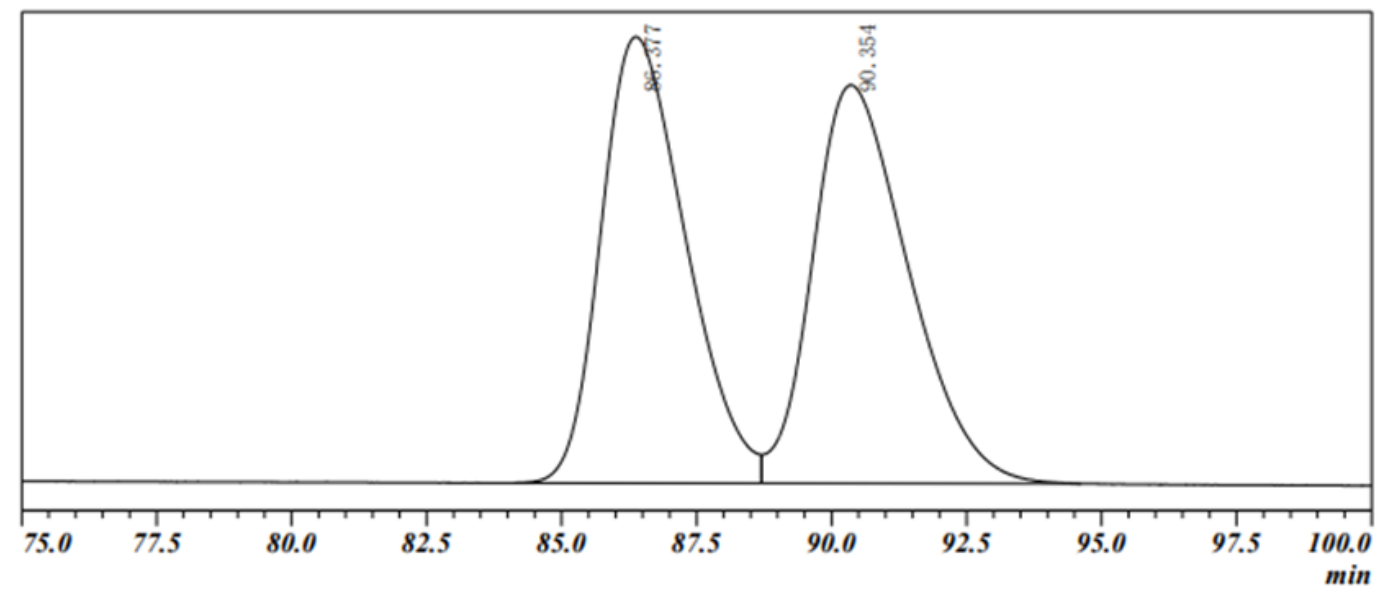

PEAK TABLE

\section{<格表>}

\begin{tabular}{|r|r|r|r|r|r|}
\hline Peak NO. & Retention time & \multicolumn{1}{|c|}{ Area } & \multicolumn{1}{c|}{ Area\% } & \multicolumn{1}{c|}{ Height } & \multicolumn{1}{|c|}{ Height $\%$} \\
\hline 1 & 86.377 & 4326177 & 49.674 & 39624 & 52.843 \\
\hline 2 & 90.354 & 4383030 & 50.326 & 35360 & 47.157 \\
\hline 计 & & 8709206 & 100.000 & 74984 & 100.000 \\
\hline
\end{tabular}

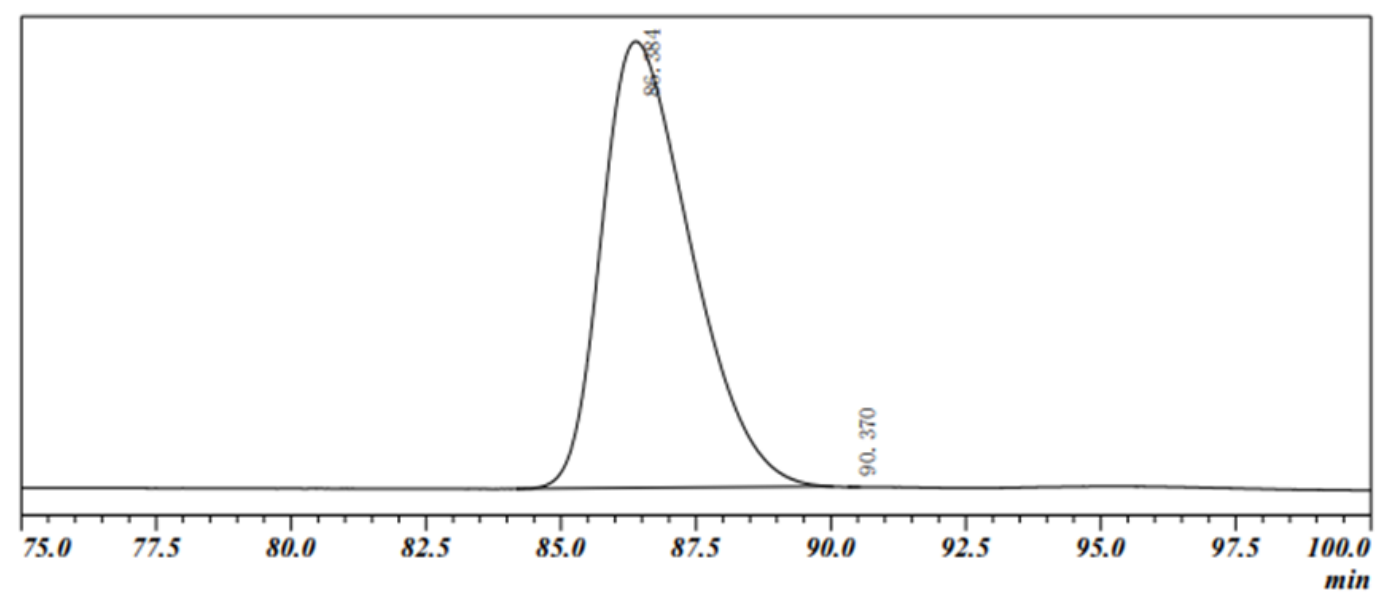

<峰表

PEAK TABLE

格灌A ChI $254 \mathrm{~nm}$

\begin{tabular}{|c|c|c|c|c|c|}
\hline Peak NO. & Retention time & Area & Area\% & Height & Height $\%$ \\
\hline$I$ & 86.384 & 6273413 & 100.000 & 54546 & 99.98 \\
\hline 2 & 90.370 & 17 & 0.000 & 7 & 0.01 \\
\hline 总咞 & & 6273429 & 100.000 & 54552 & 100.0 \\
\hline
\end{tabular}



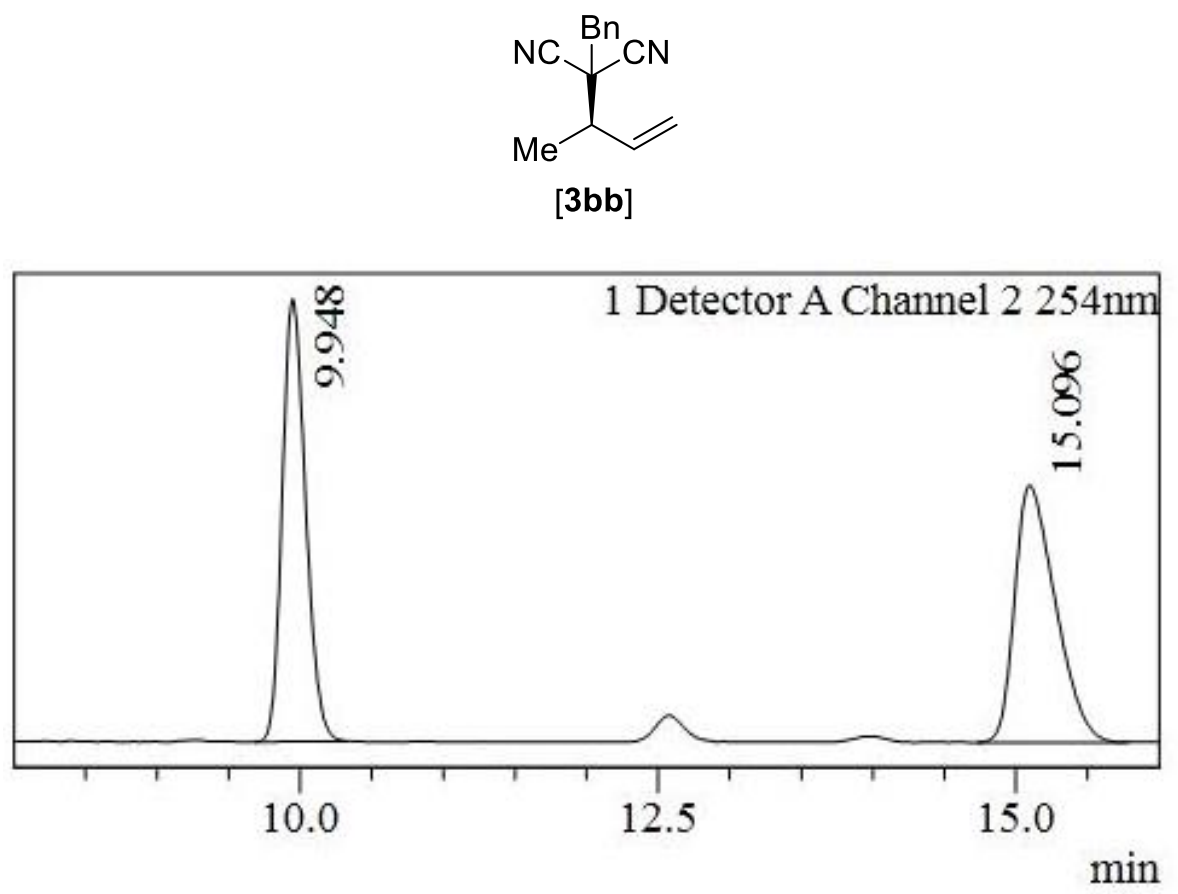

Peak Table

\begin{tabular}{|c|c|c|c|c|c|c|}
\hline \multicolumn{7}{|c|}{ Detector A Channel $2254 \mathrm{~nm}$} \\
\hline Peak\# & Ret. Time & Area & Height & Area $\%$ & Conc. & Name \\
\hline 1 & 9.948 & 98529 & 8677 & 49.661 & 49.661 & \\
\hline 2 & 15.096 & 99874 & 5039 & 50.339 & 50.339 & \\
\hline Tota & & 198403 & 13716 & 100.000 & & \\
\hline
\end{tabular}
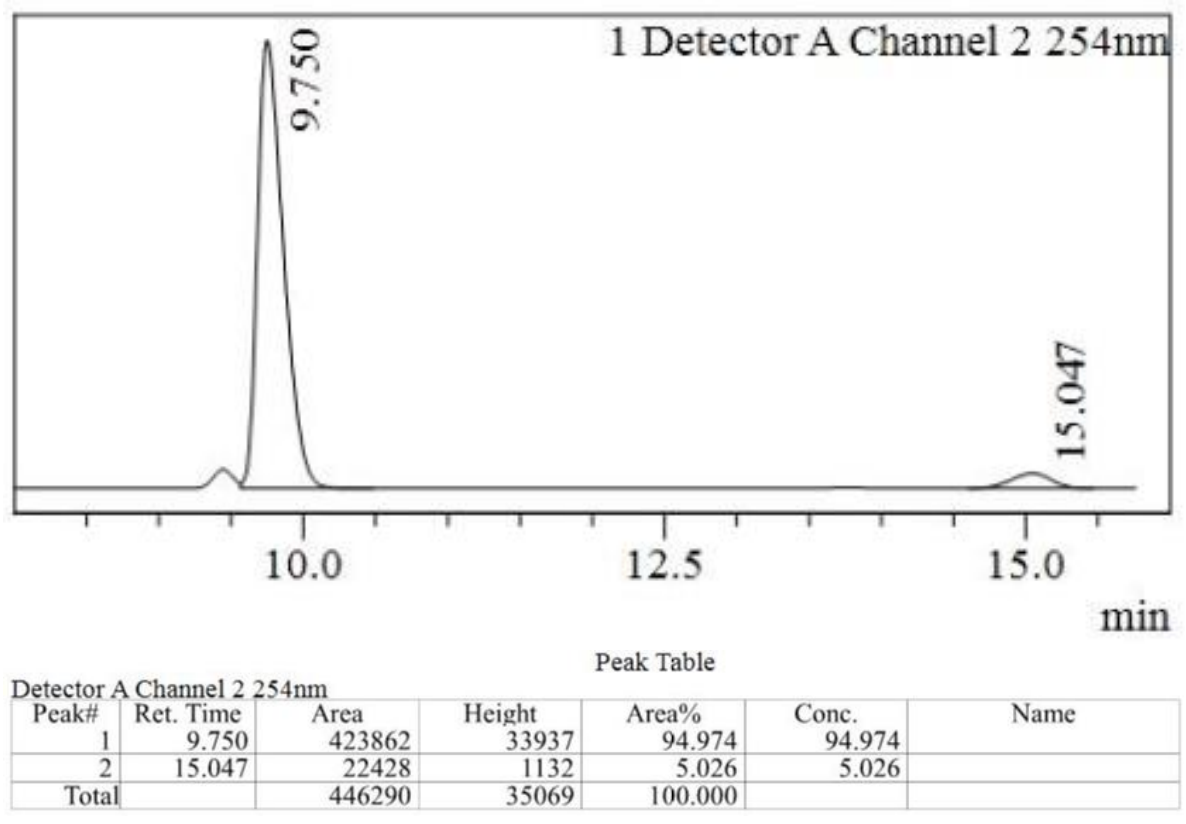

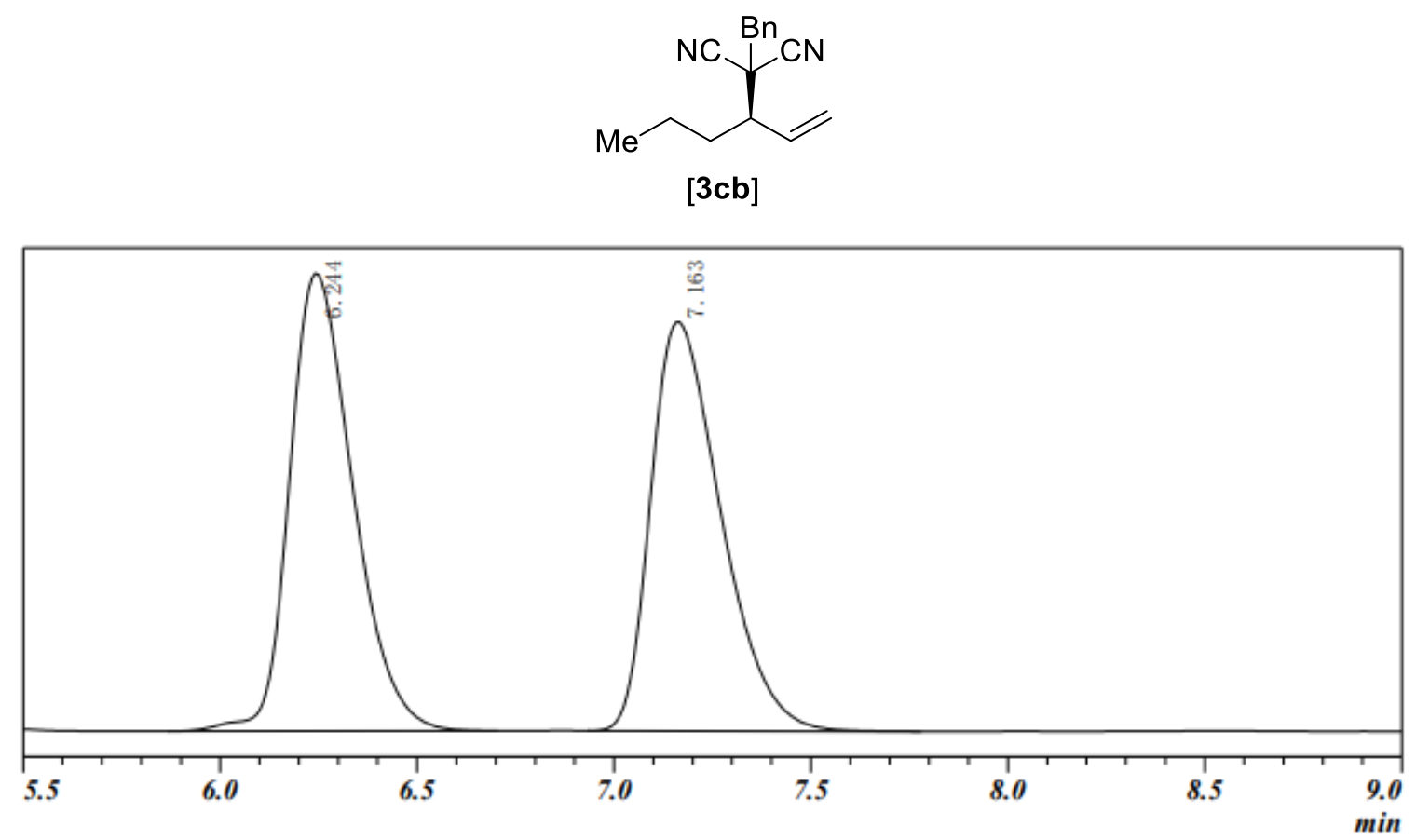

PEAK TABLE

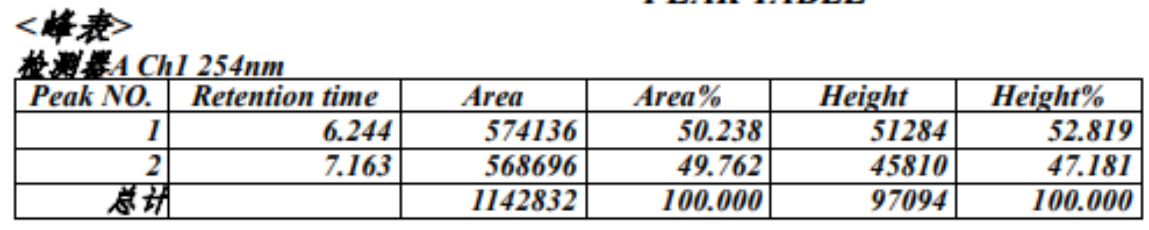

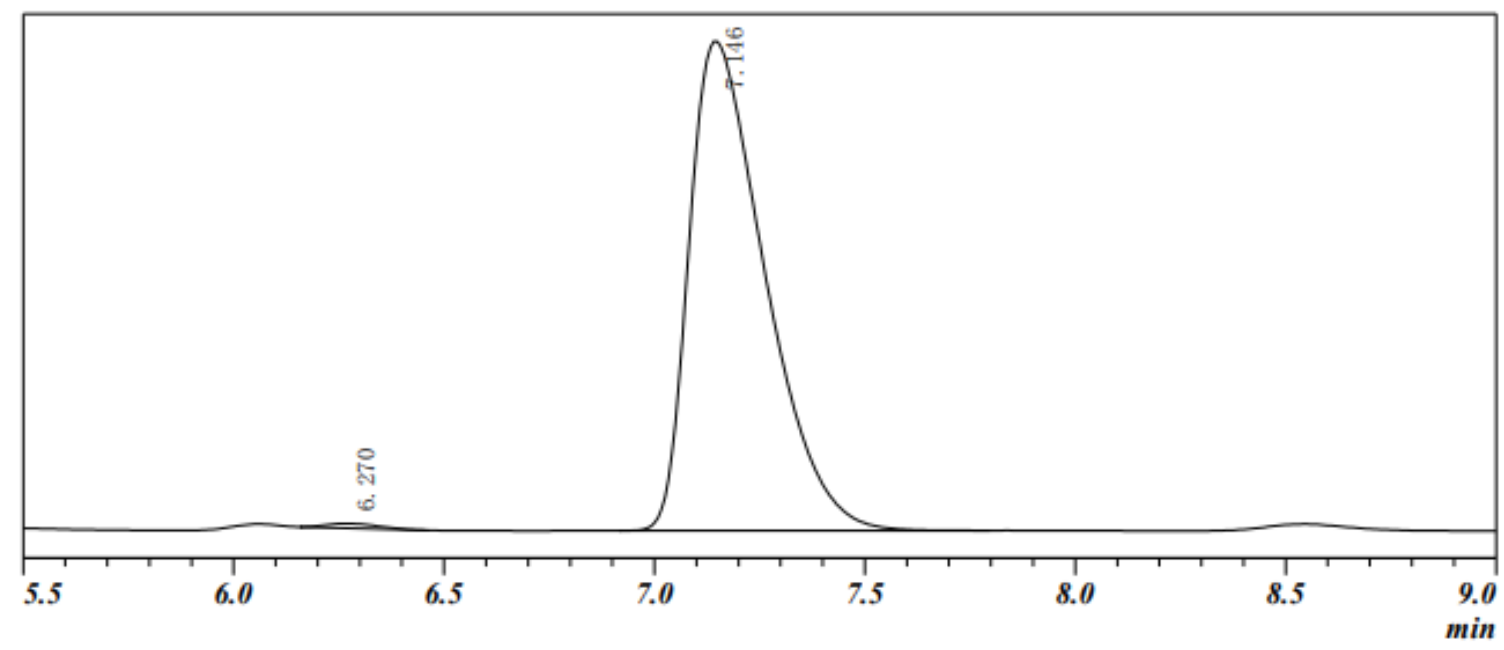

PEAK TABLE

表然A ChI 254nm

\begin{tabular}{|r|r|r|r|r|r|}
\hline Peak NO. & Retention time & \multicolumn{1}{c|}{ Area } & \multicolumn{1}{c|}{ Area\% } & \multicolumn{1}{c|}{ Height } & \multicolumn{1}{c|}{ Height $\%$} \\
\hline 1 & 6.270 & 5628 & 0.618 & 679 & 0.932 \\
\hline 2 & 7.146 & 904633 & 99.382 & 72211 & 99.068 \\
\hline 总 & & 910261 & 100.000 & 72890 & 100.000 \\
\hline
\end{tabular}




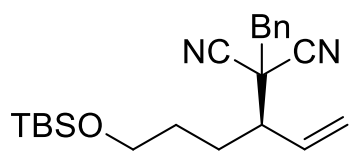

[3db]

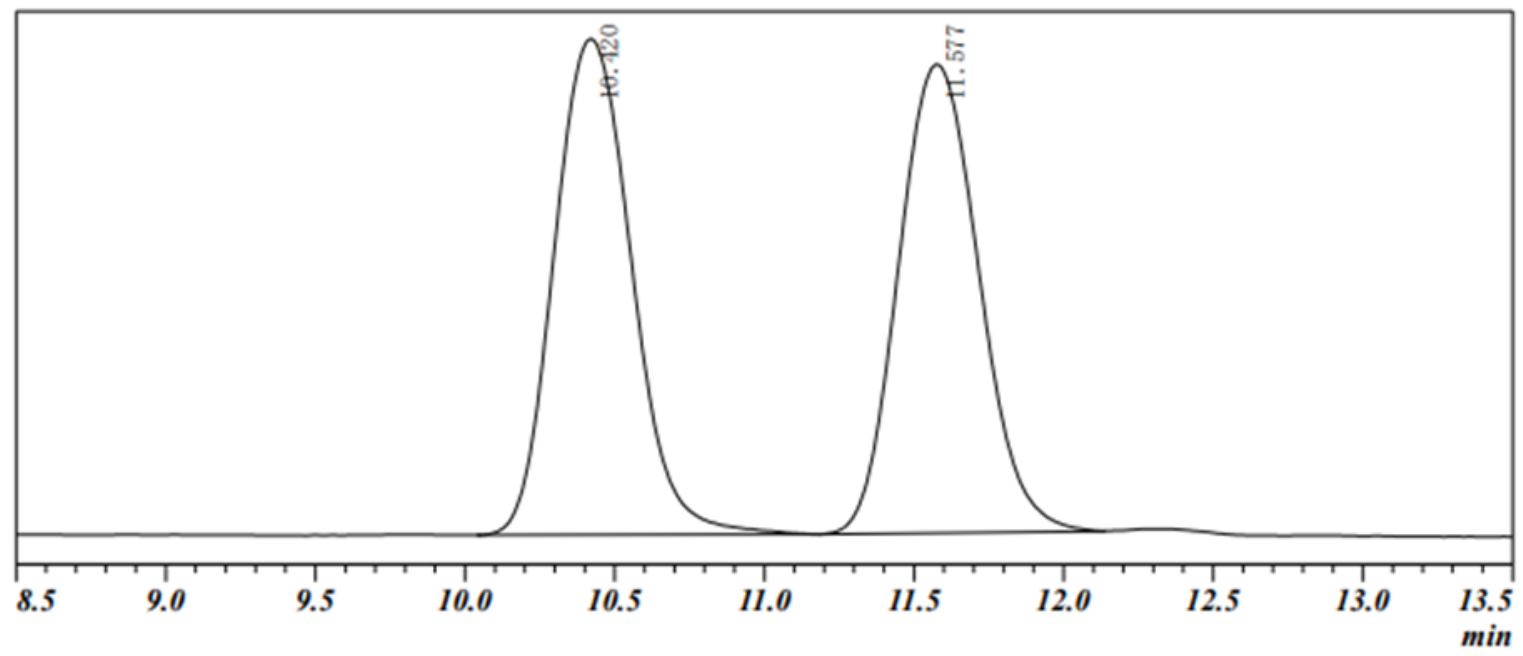

PEAK TABLE

\begin{tabular}{|c|c|c|c|c|c|}
\hline Peak NO. & Retention time & Area & Area $\%$ & Height & Height $\%$ \\
\hline 1 & 10.420 & 279693 & 50.465 & 15548 & 51.405 \\
\hline-1 & 11.577 & 274537 & 49.535 & 14698 & 48.595 \\
\hline 总线 & & 554230 & 100.000 & 30245 & 100.000 \\
\hline
\end{tabular}

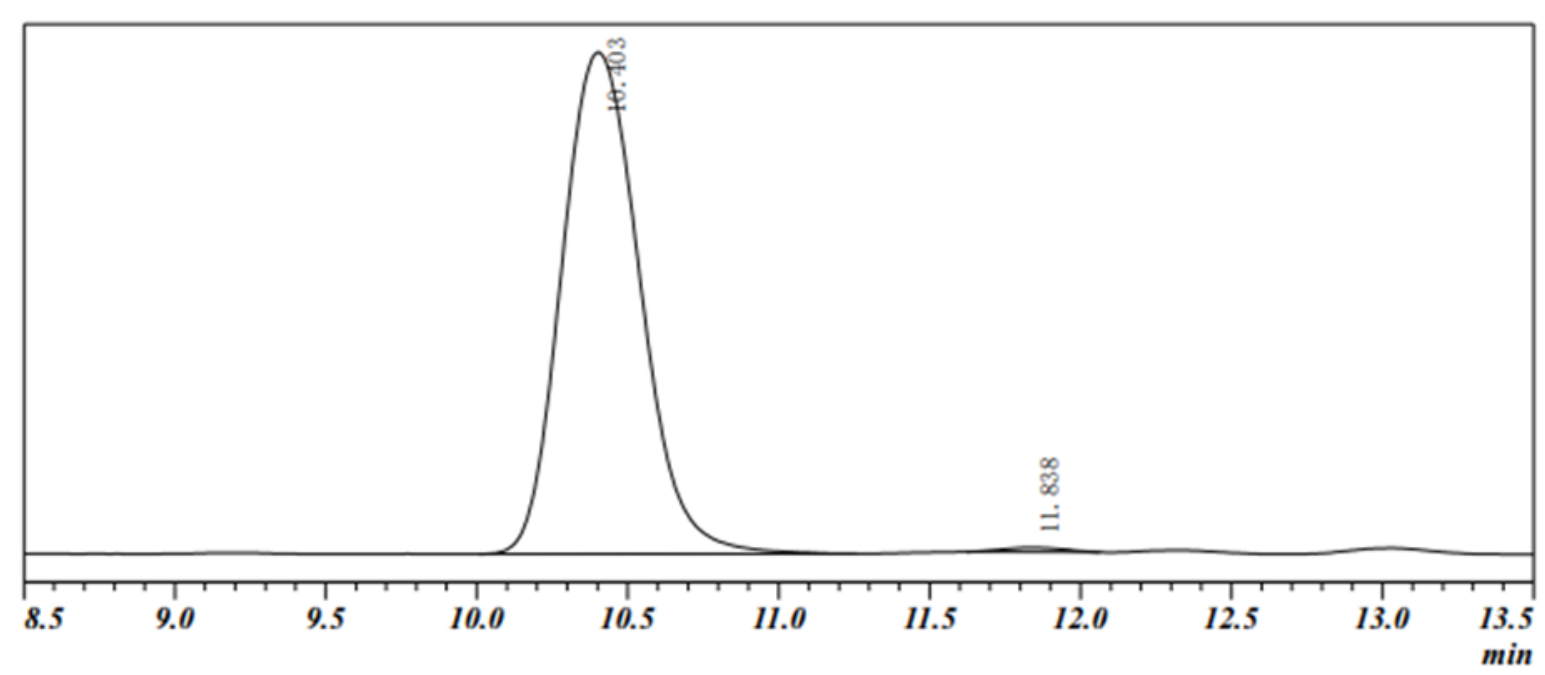

PEAK TABLE

\begin{tabular}{|c|c|c|c|c|c|}
\hline Peak NO. & Retention time & Area & Area\% & Height & Height $\%$ \\
\hline 1 & 10.403 & 689112 & 99.323 & 38258 & 99.147 \\
\hline 2 & 11.838 & 4700 & 0.677 & 329 & 0.853 \\
\hline 总计 & & 693812 & 100.000 & 38588 & 100.000 \\
\hline
\end{tabular}




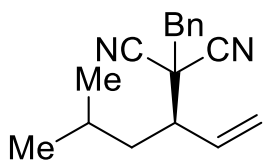

[3eb]

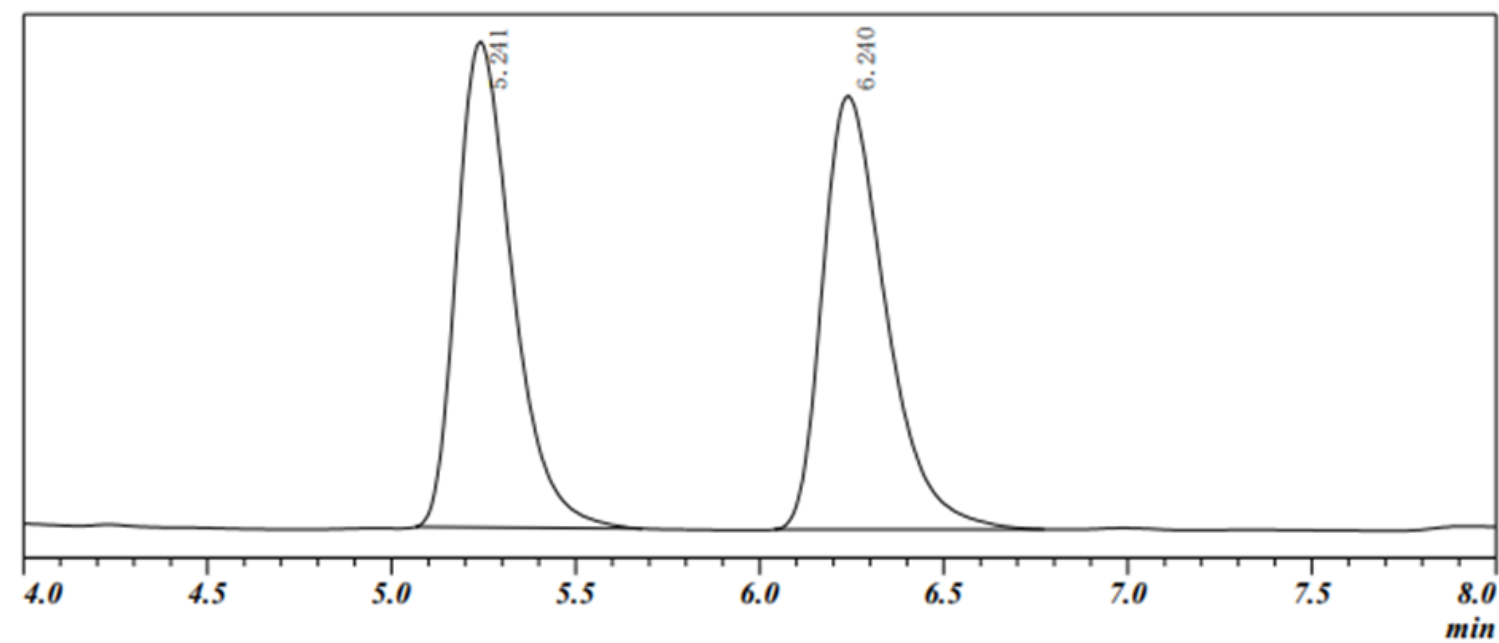

PEAK TABLE

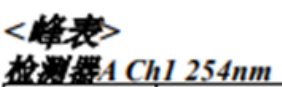

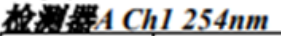

\begin{tabular}{|r|r|r|r|r|r|}
\hline Peak NO. & Retention time & \multicolumn{1}{c|}{ Area } & \multicolumn{1}{c|}{ Area\% } & \multicolumn{1}{c|}{ Height } & \multicolumn{1}{|c|}{ Height $\%$} \\
\hline 1 & 5.241 & 475890 & 49.659 & 45227 & 52.835 \\
\hline 2 & 6.240 & 482431 & 50.341 & 40373 & 47.165 \\
\hline 尊 & & 958321 & 100.000 & 85601 & 100.000 \\
\hline
\end{tabular}

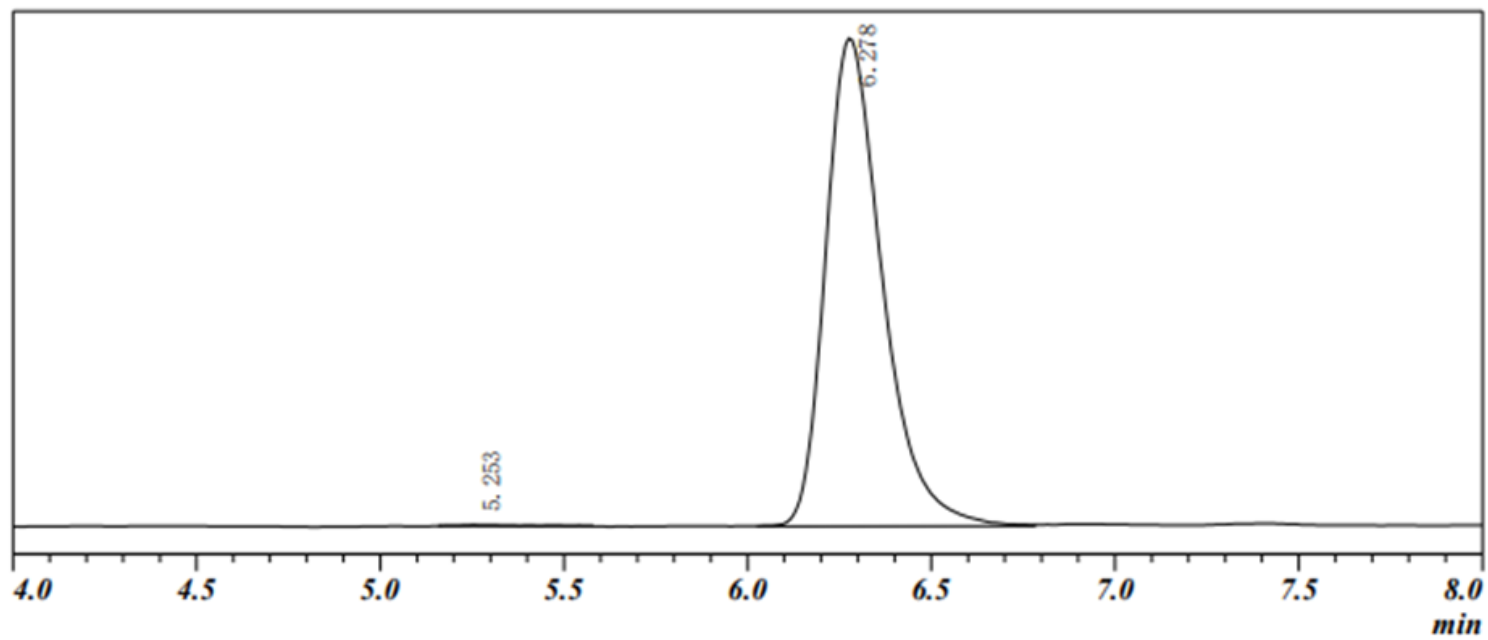

PEAK TABLE

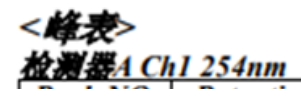

\begin{tabular}{|r|r|r|r|r|r|}
\hline Peak NO. & Retention time & \multicolumn{1}{c|}{ Area } & \multicolumn{1}{c|}{ Area\% } & \multicolumn{1}{c|}{ Height } & \multicolumn{1}{c|}{ Height\% } \\
\hline 1 & 5.253 & 334 & 0.182 & 32 & 0.188 \\
\hline 2 & 6.278 & 182937 & 99.818 & 16976 & 99.812 \\
\hline 总 & & 183270 & 100.000 & 17008 & 100.000 \\
\hline
\end{tabular}




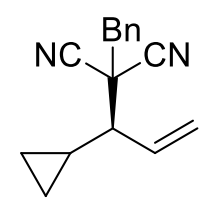

[3fb]

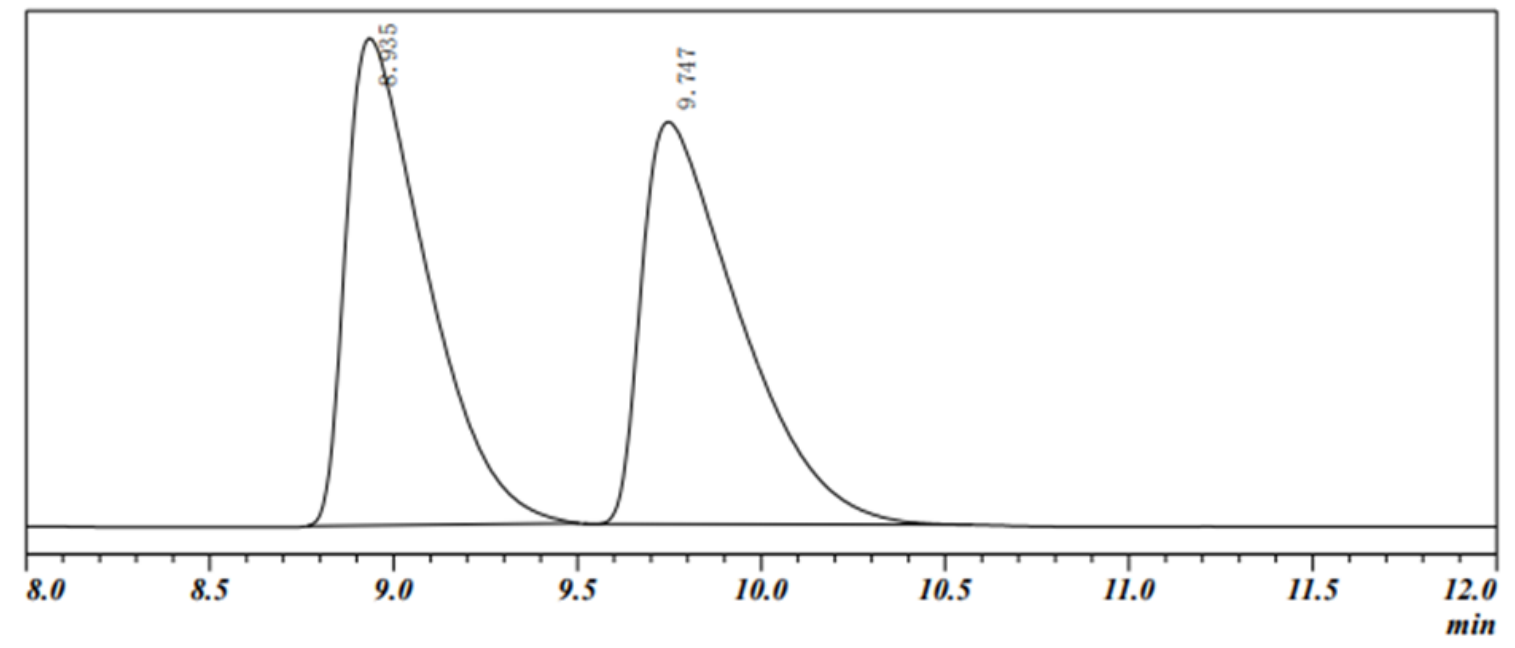

PEAK TABLE

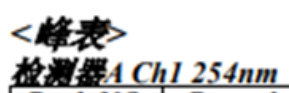

\begin{tabular}{|r|r|r|r|r|r|}
\hline Peak NO. & Retention time & \multicolumn{1}{|c|}{ Area } & \multicolumn{1}{c|}{ Area $\%$} & \multicolumn{1}{c|}{ Height } & \multicolumn{1}{c|}{ Height $\%$} \\
\hline 1 & 8.935 & 1249131 & 49.978 & 82062 & 54.789 \\
\hline 2 & 9.747 & 1250224 & 50.022 & 67717 & 45.211 \\
\hline 㒸 & & 2499355 & 100.000 & 149779 & 100.000 \\
\hline
\end{tabular}

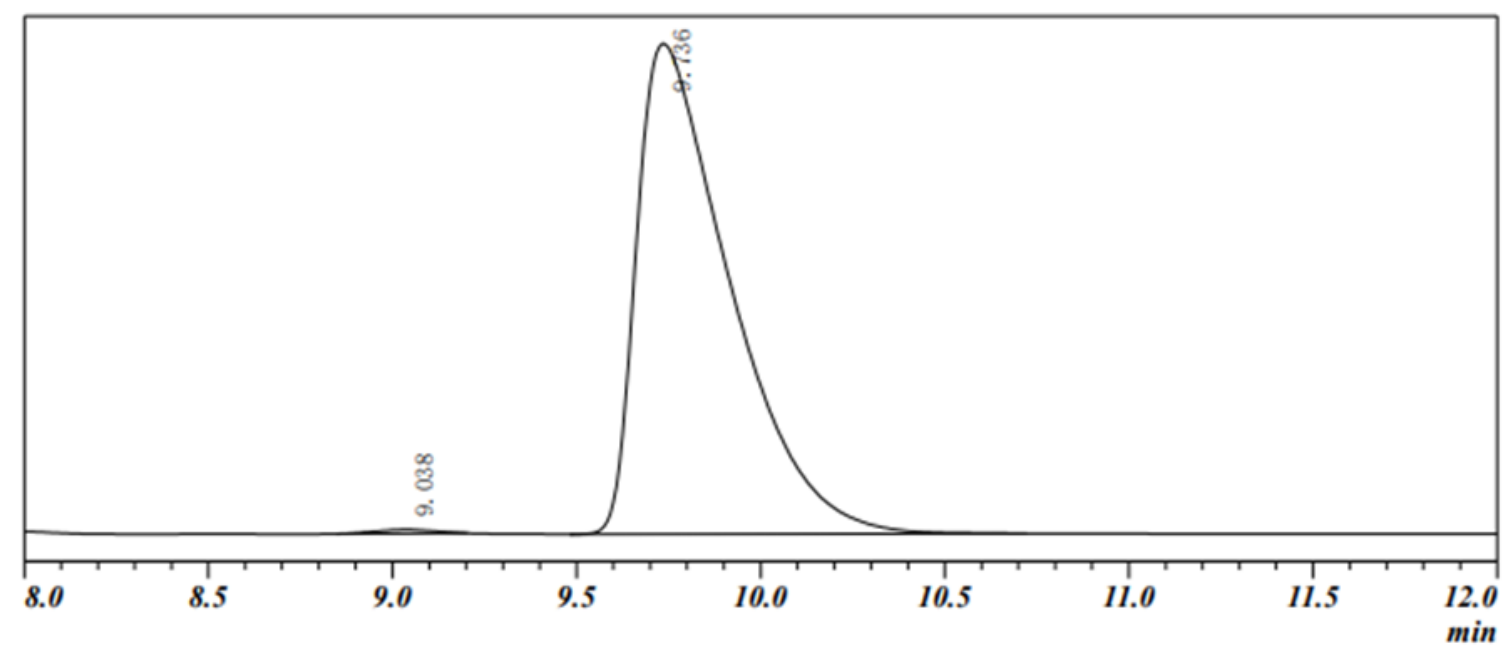

PEAK TABLE

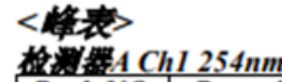

\begin{tabular}{|c|c|c|c|c|c|}
\hline Peak NO. & Retention time & Area & Area\% & Height & Height $\%$ \\
\hline$I$ & 9.038 & 5008 & 0.490 & 456 & 0.782 \\
\hline 2 & 9.736 & 1017316 & 99.510 & 57887 & 99.218 \\
\hline 总计 & & 1022325 & 100.000 & 58343 & 100.000 \\
\hline
\end{tabular}




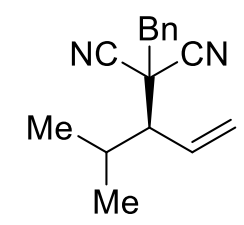

[3gb]

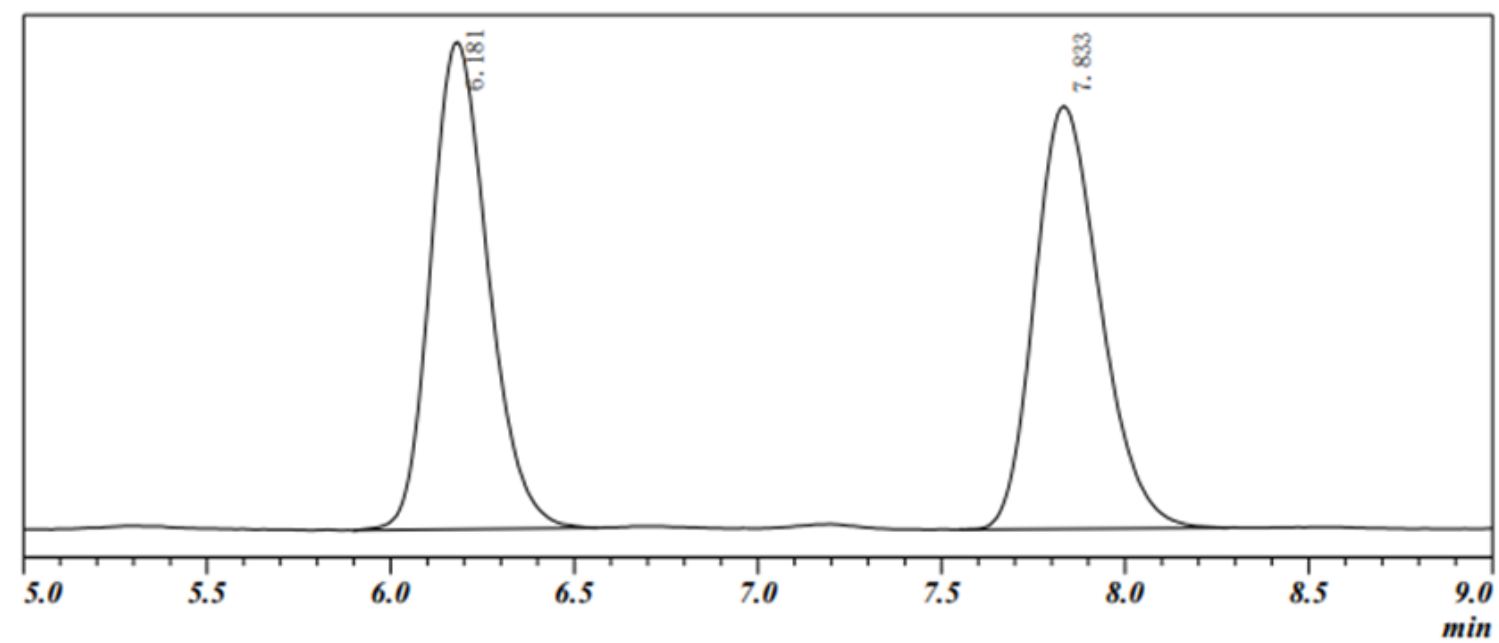

PEAK TABLE

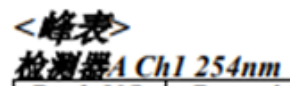

\begin{tabular}{|r|r|r|r|r|r|}
\hline Peak NO. & Retention time & \multicolumn{1}{c|}{ Area } & \multicolumn{1}{|c|}{ Area\% } & \multicolumn{1}{c|}{ Height } & \multicolumn{1}{|c|}{ Height\% } \\
\hline 1 & 6.181 & 98978 & 50.066 & 9126 & 53.522 \\
\hline 2 & 7.833 & 98717 & 49.934 & 7925 & 46.478 \\
\hline 总 & & 197695 & 100.000 & 17051 & 100.000 \\
\hline
\end{tabular}

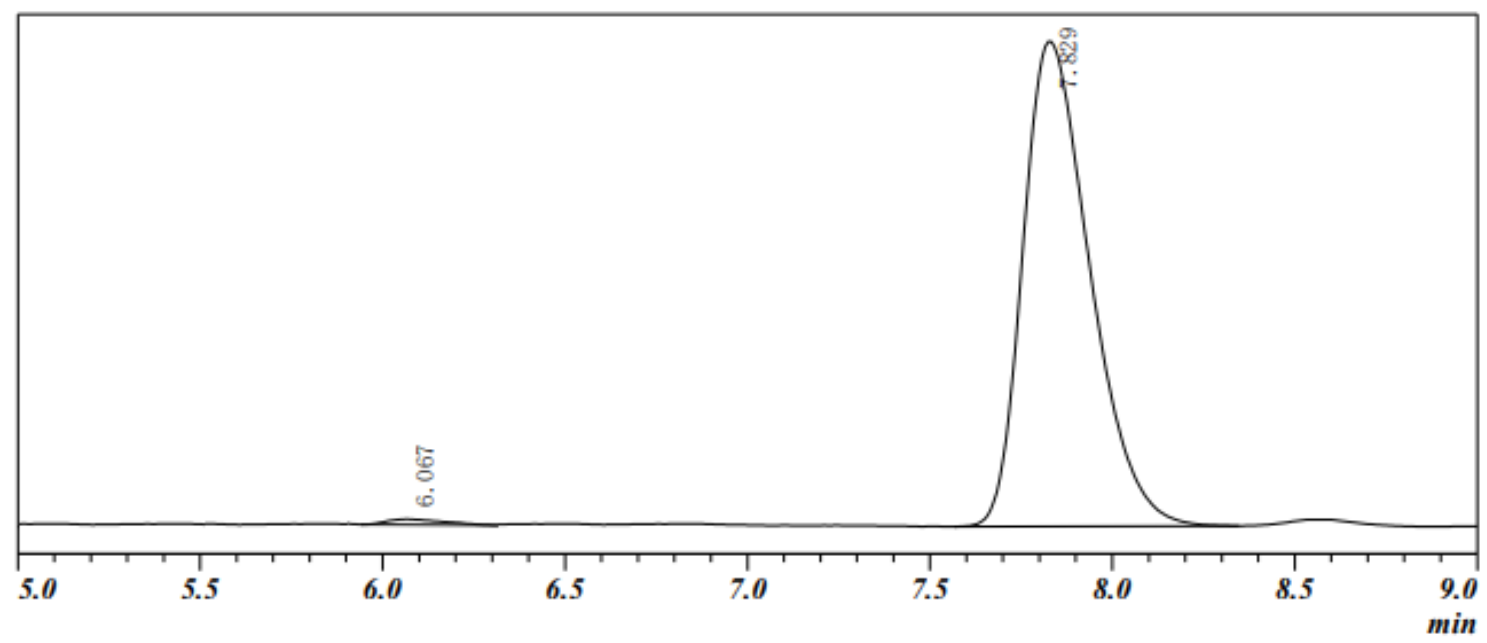

PEAK TABLE

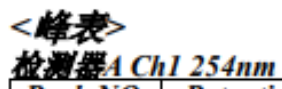

\begin{tabular}{|c|c|c|c|c|c|}
\hline Peak NO. & Retention time & Area & Area $\%$ & Height & Height $\%$ \\
\hline$I$ & 6.067 & 3004 & 0.983 & 278 & 1.159 \\
\hline 2 & 7.829 & 302511 & 99.017 & 23664 & 98.841 \\
\hline 意识 & & 305514 & 100.000 & 23942 & 100.000 \\
\hline
\end{tabular}




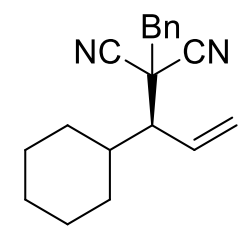

[3hb]

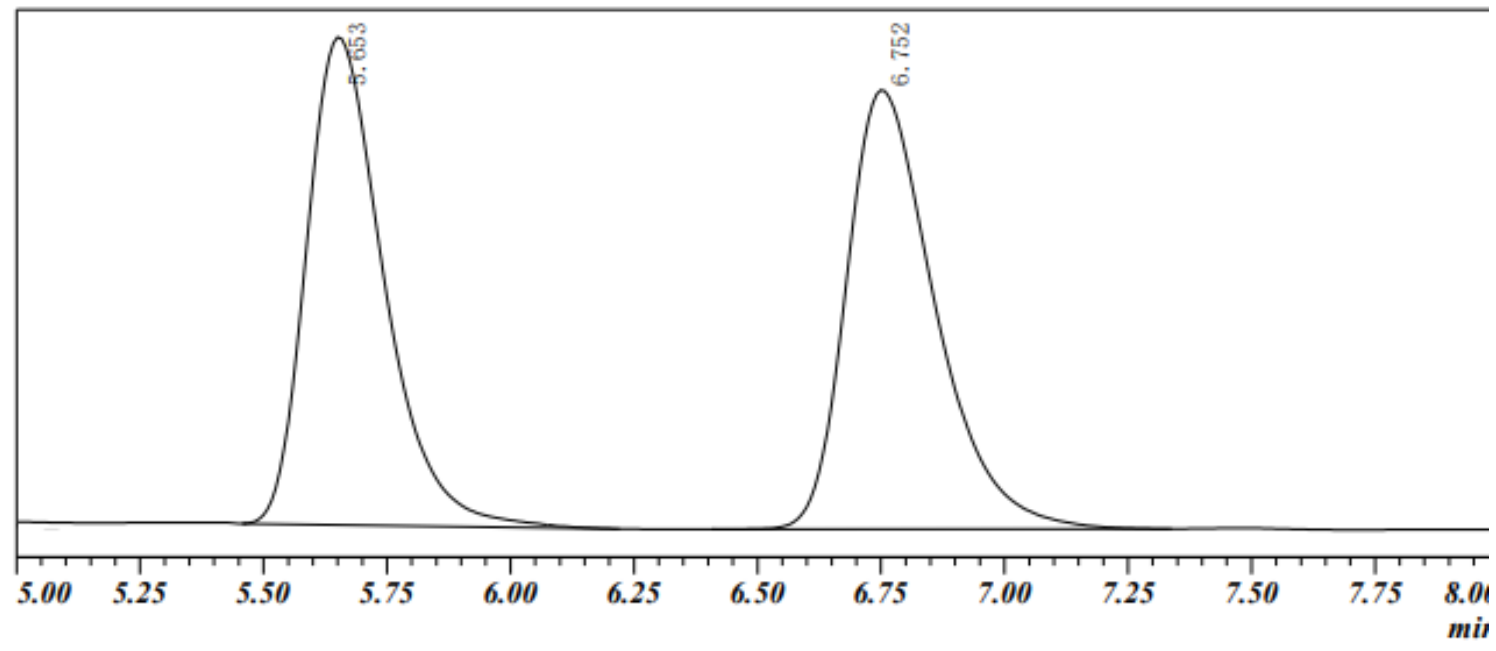

PEAK TABLE

<峰表

\begin{tabular}{|l|l|l|l|l|l|}
\hline Peak NO. & Retention time & Area & Area $\%$ & Height & Height $\%$ \\
\hline
\end{tabular}

\begin{tabular}{|c|c|c|c|c|c|}
\hline . & & & & & \\
\hline$\frac{1}{1}$ & 5.653 & 442411 & 49.206 & 40640 & 52.626 \\
\hline$\frac{2}{8}$ & 6.752 & 456690 & 50.794 & 36584 & 47.374 \\
\hline S试 & & 899100 & 100.000 & 77224 & 100.000 \\
\hline
\end{tabular}

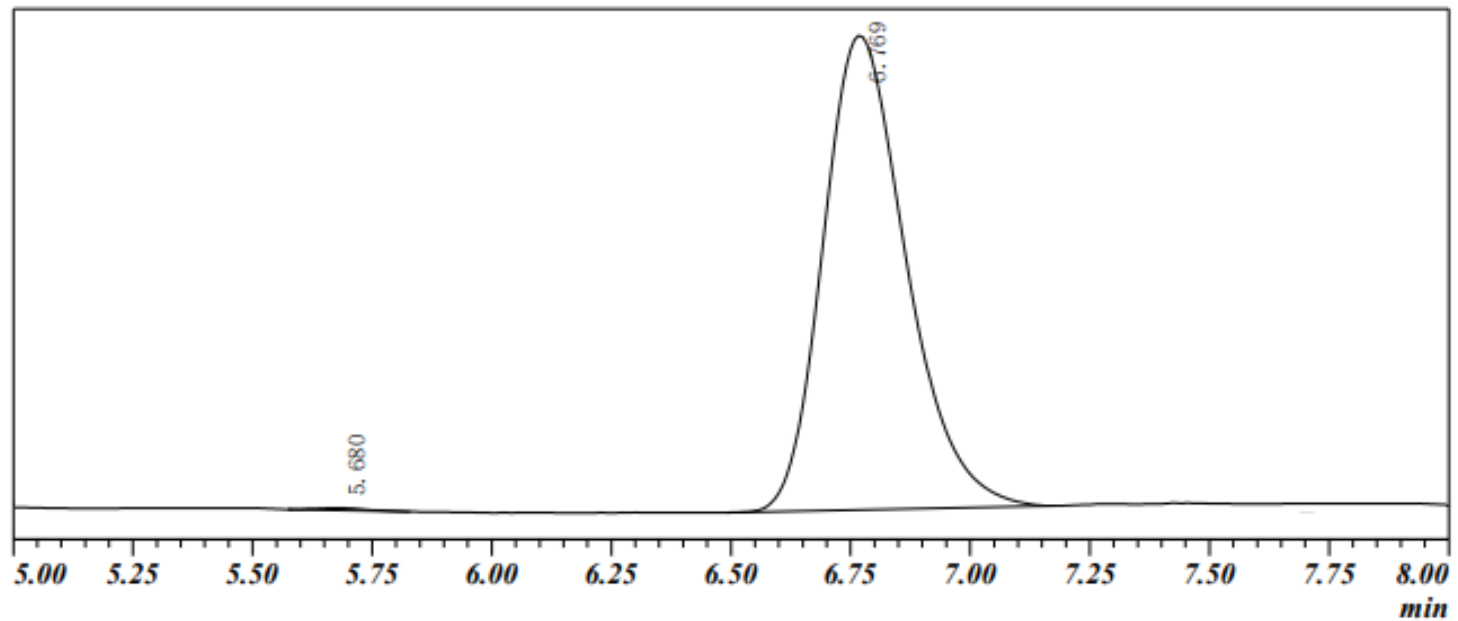

PEAK TABLE

\section{<数哀>}

\begin{tabular}{|r|r|r|r|r|r|}
\hline Peak NO. & Retention time & \multicolumn{1}{c|}{ Area } & \multicolumn{1}{c|}{ Area\% } & \multicolumn{1}{c|}{ Height } & \multicolumn{1}{c|}{ Height $\%$} \\
\hline 1 & 5.680 & 415 & 0.278 & 61 & 0.497 \\
\hline 2 & 6.769 & 149005 & 99.722 & 12294 & 99.503 \\
\hline 总 & & 149420 & 100.000 & 12355 & 100.000 \\
\hline
\end{tabular}




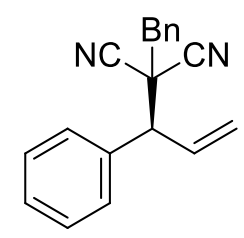

[3ib]

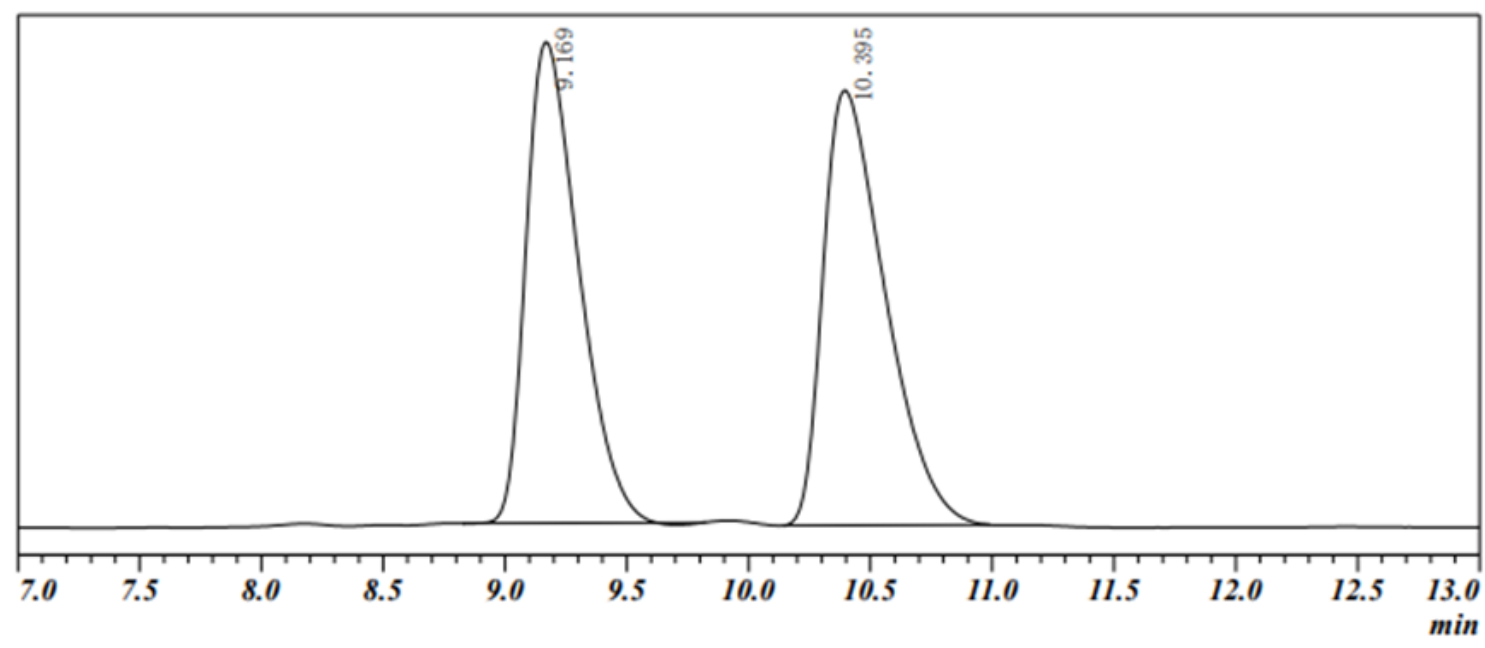

PEAK TABLE

\section{<都衣}

\begin{tabular}{|r|r|r|r|r|r|}
\hline Peak NO. & Retention time & \multicolumn{1}{c|}{ Area } & \multicolumn{1}{c|}{ Area\% } & \multicolumn{1}{c|}{ Height } & \multicolumn{1}{c|}{ Height $\%$} \\
\hline 1 & 9.169 & 1251278 & 48.382 & 83259 & 52.523 \\
\hline 2 & 10.395 & 1334946 & 51.618 & 75259 & 47.477 \\
\hline 总话 & & 2586224 & 100.000 & 158518 & 100.000 \\
\hline
\end{tabular}

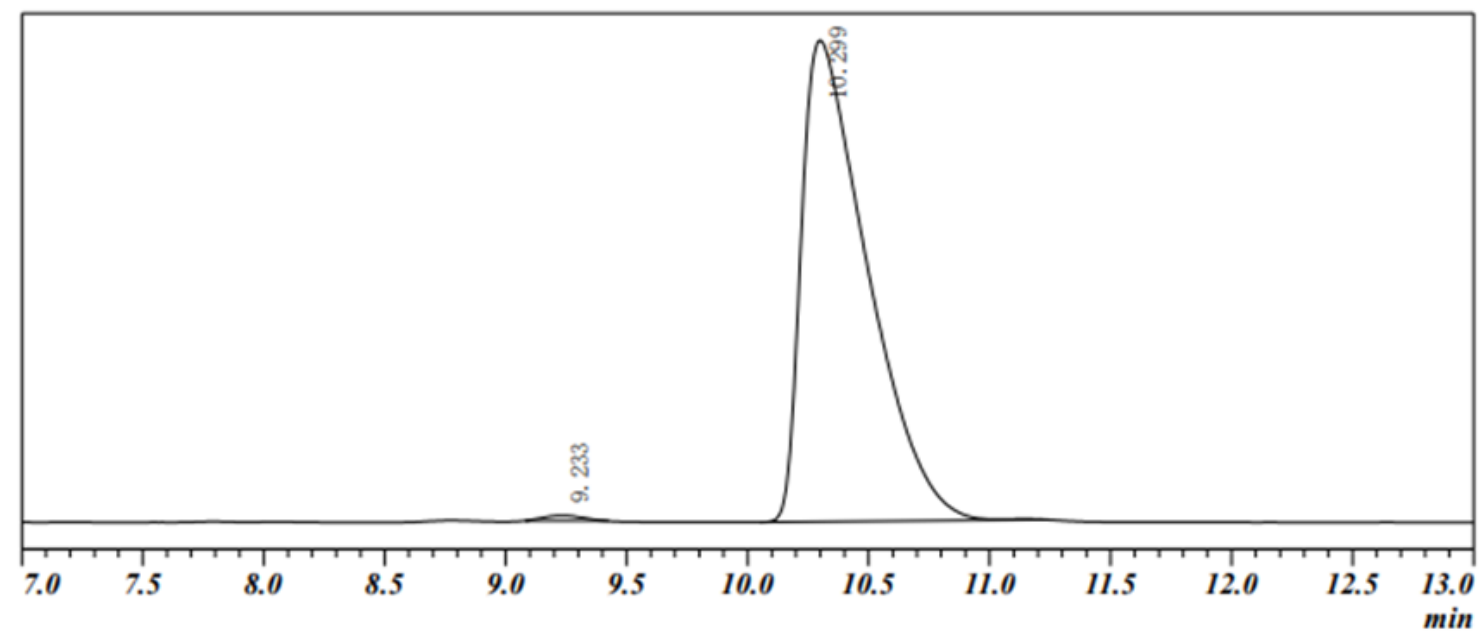

PEAK TABLE

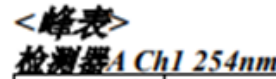

\begin{tabular}{|r|r|r|r|r|r|}
\hline Peak NO. & Retention time & \multicolumn{1}{c|}{ Area } & \multicolumn{1}{c|}{ Area\% } & \multicolumn{1}{c|}{ Height } & \multicolumn{1}{c|}{ Height\% } \\
\hline 1 & 9.233 & 17864 & 0.627 & 1628 & 1.079 \\
\hline 2 & 10.299 & 2832343 & 99.373 & 149195 & 98.921 \\
\hline 总话 & & 2850207 & 100.000 & 150823 & 100.000 \\
\hline
\end{tabular}




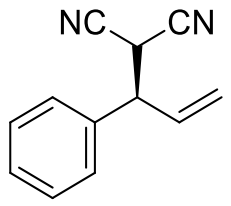

[3ja]

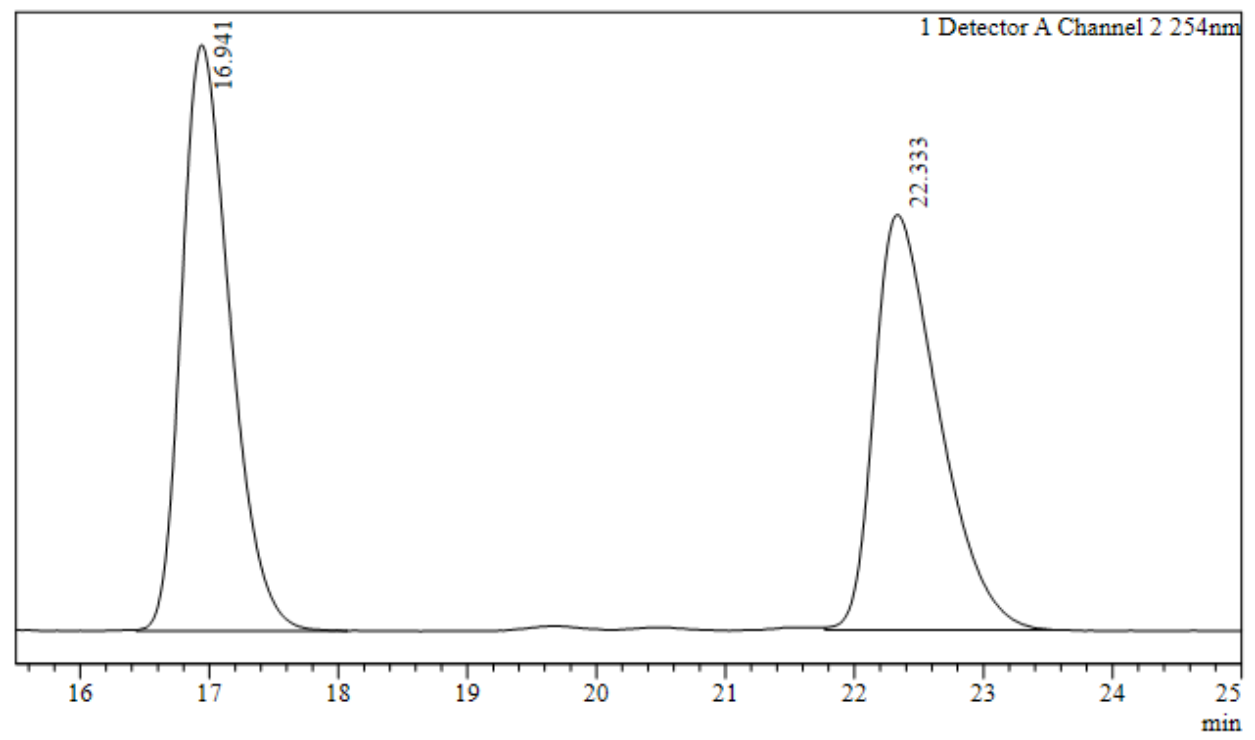

Peak Table
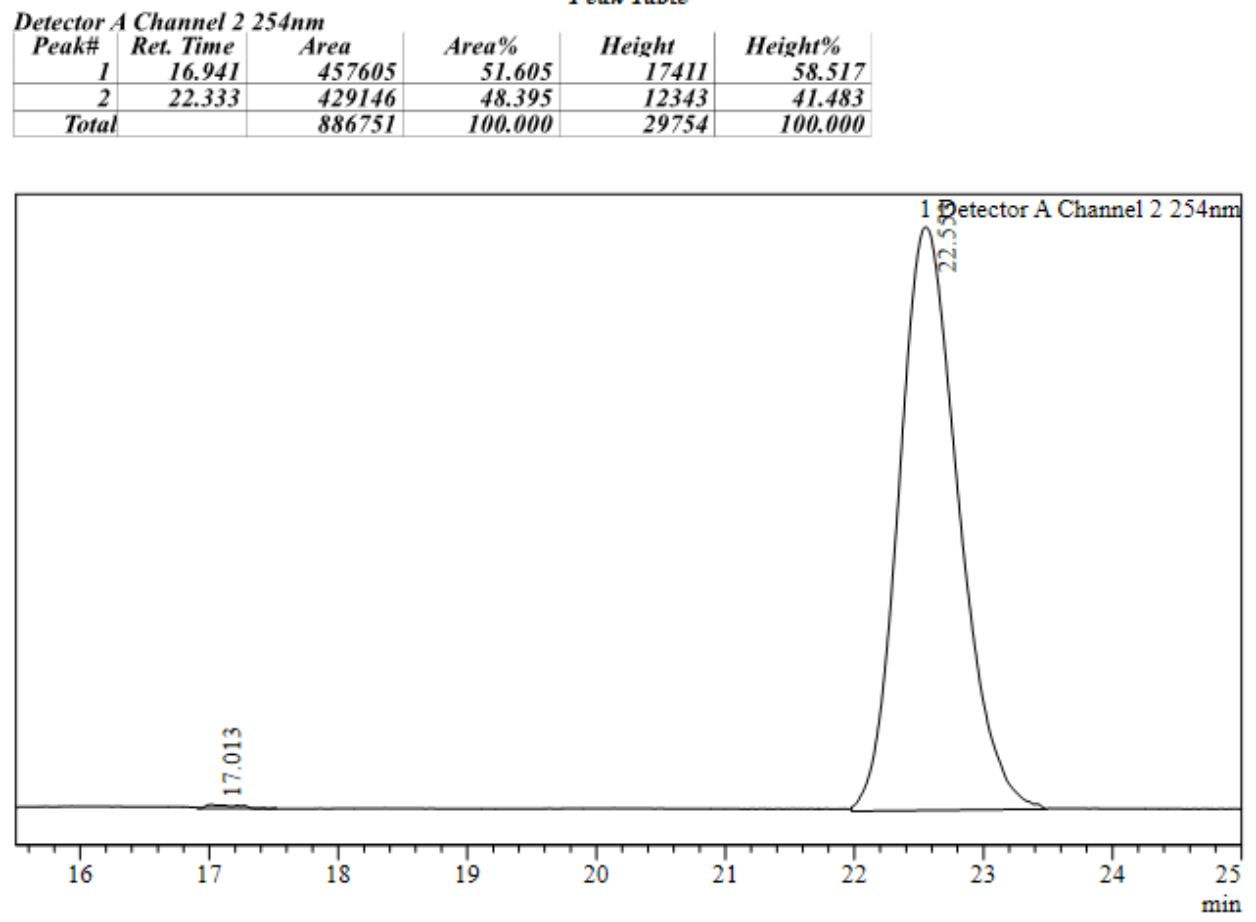

Peak Table

\begin{tabular}{|c|c|c|c|c|c|}
\hline \multicolumn{3}{|c|}{ Detector A Channel 2} & \multicolumn{3}{|c|}{ Peak To } \\
\hline Peak\# & Ret. Time & Area & Area $\%$ & Height & Height $\%$ \\
\hline$\frac{1}{2}$ & 17.013 & $\begin{array}{r}238 \\
70817\end{array}$ & $\begin{array}{r}0.297 \\
99.703\end{array}$ & 14 & 0.578 \\
\hline Total & 22.030 & 80055 & $\begin{array}{l}99.703 \\
100.000\end{array}$ & 2504 & 100.000 \\
\hline
\end{tabular}




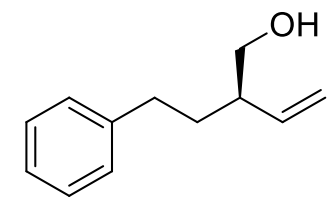

[4]

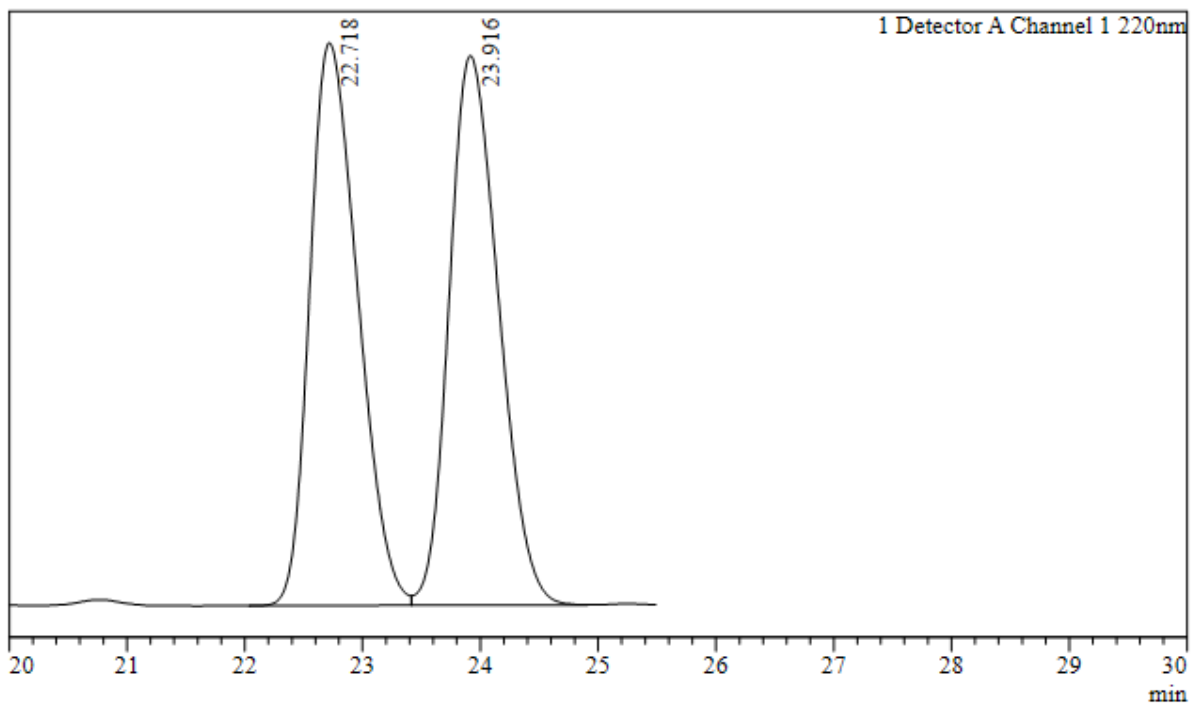

Peak Table

\begin{tabular}{|c|c|c|c|c|c|c|}
\hline Detector & Channel 1 & & & & & \\
\hline Peak\# & $\begin{array}{r}\text { Ret. Time } \\
22.718\end{array}$ & $\begin{array}{l}\text { Area } \\
2737301\end{array}$ & $\begin{array}{l}\text { Height } \\
96511\end{array}$ & $\begin{array}{l}\text { Area } \% \\
\quad 49.859\end{array}$ & $\begin{array}{l}\text { Conc. } \\
49.859\end{array}$ & Name \\
\hline 2 & 23.916 & 2752797 & 94237 & 50.141 & 50.141 & \\
\hline Total & & 5490097 & 190748 & 100.000 & & \\
\hline
\end{tabular}

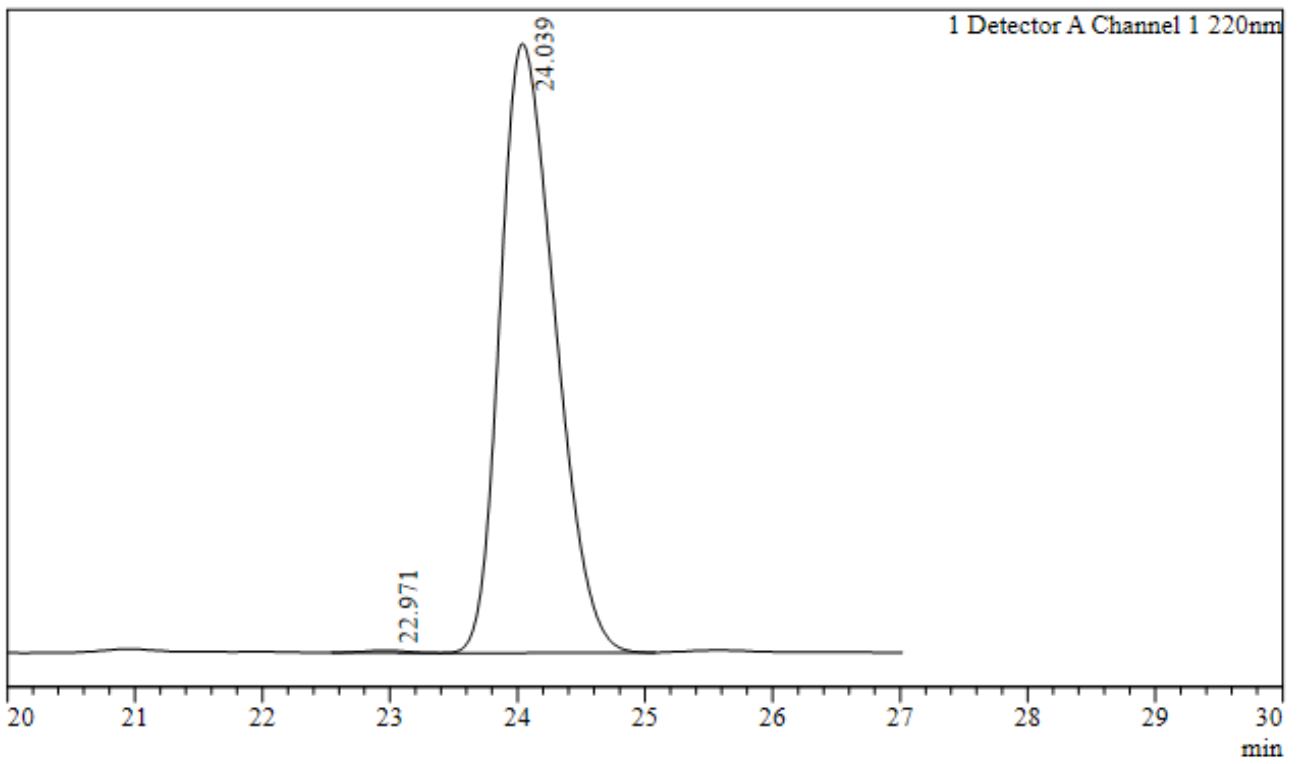

Peak Table

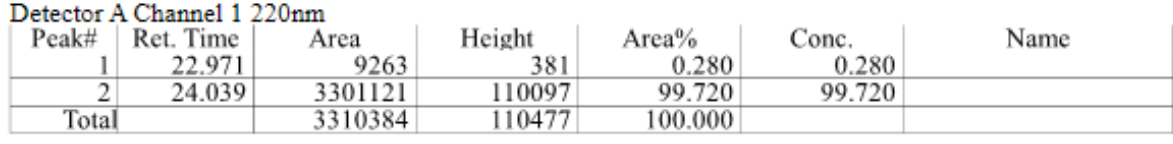




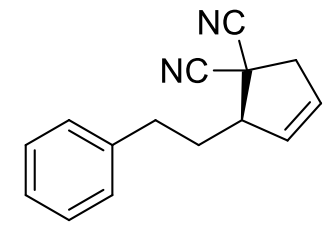

[5]

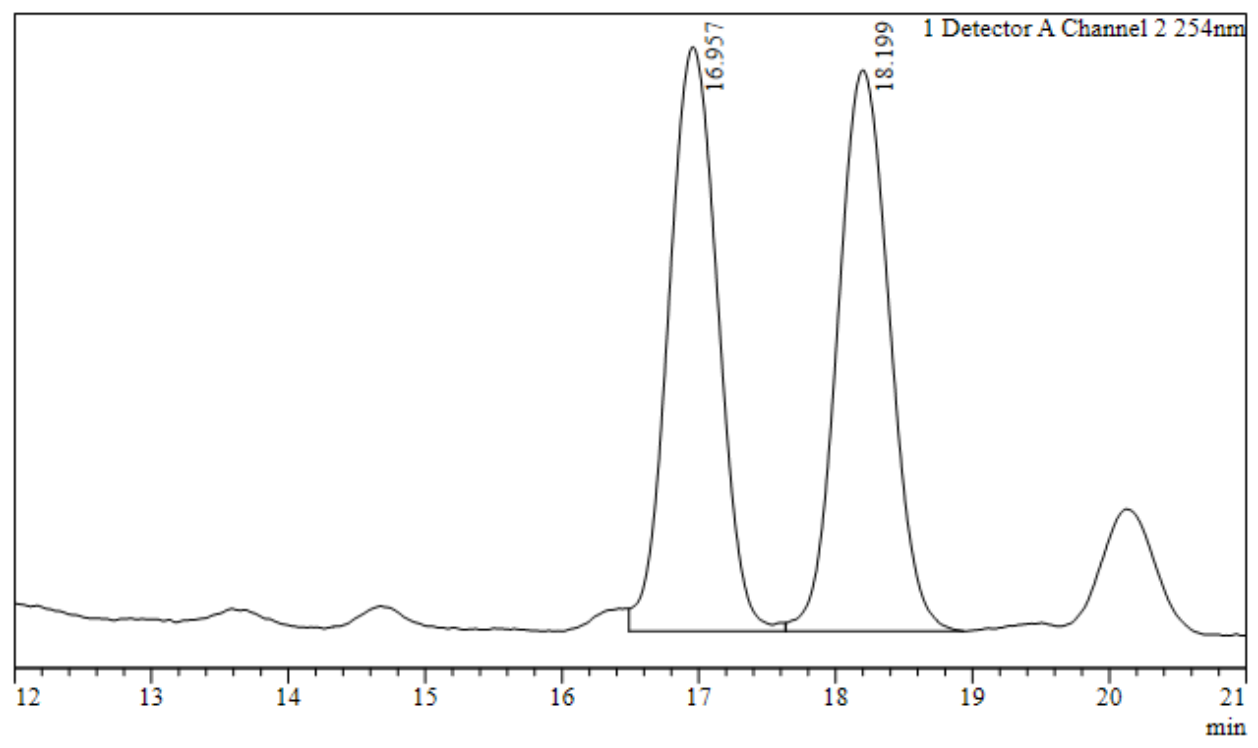

Peak Table
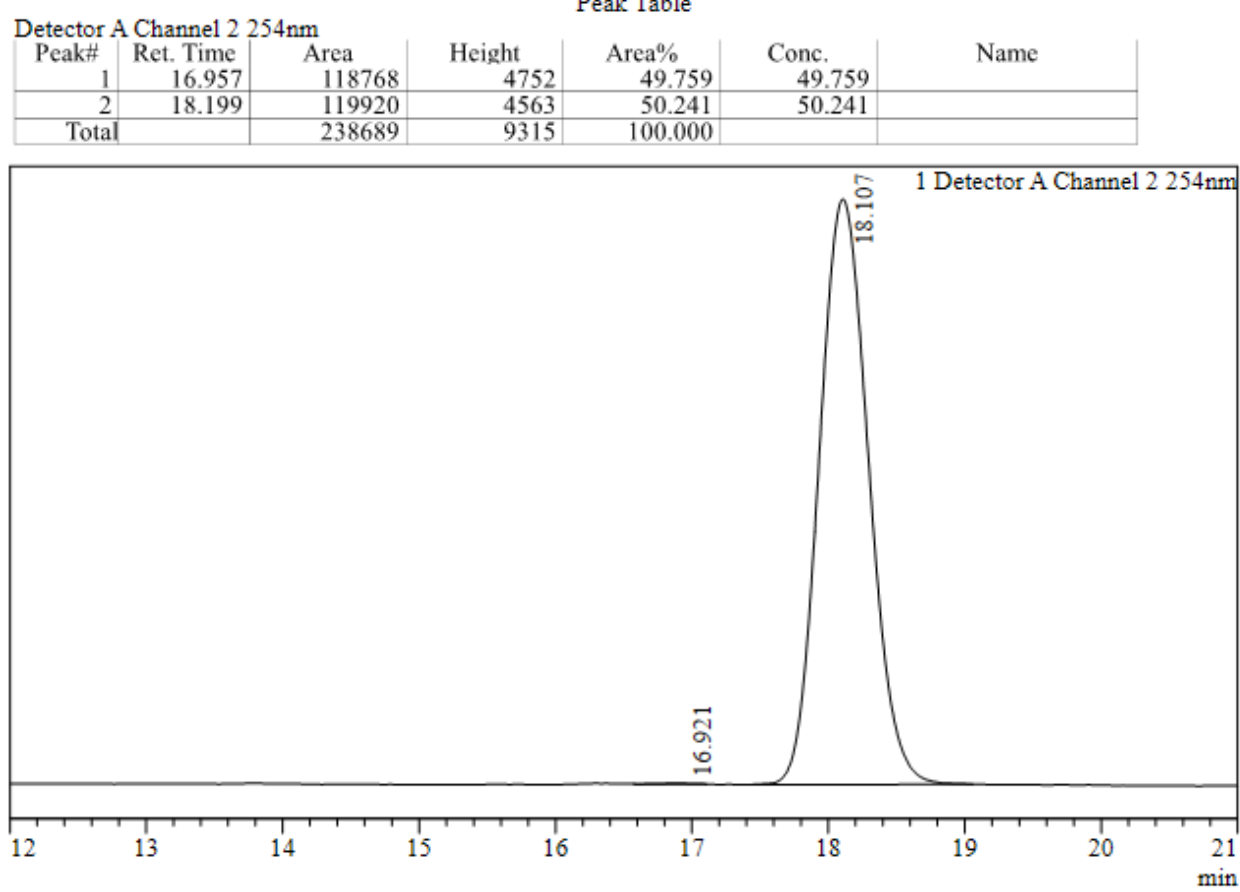

Peak Table

\begin{tabular}{|c|c|c|c|c|c|c|}
\hline \multicolumn{7}{|c|}{ Detector A Channel $2254 \mathrm{~nm}$} \\
\hline $\begin{array}{r}\text { Peak\# } \\
1\end{array}$ & $\begin{array}{r}\text { Ret. Time } \\
16.921 \\
\end{array}$ & $\begin{array}{c}\text { Area } \\
\end{array}$ & $\begin{array}{l}\text { Height } \\
\end{array}$ & $\begin{array}{l}\text { Area } \% \\
0.104\end{array}$ & $\begin{array}{l}\text { Conc. } \\
0.104\end{array}$ & Name \\
\hline 2 & 18.107 & 745205 & 29643 & 99.896 & 99.896 & \\
\hline Total & & 745978 & 29691 & 100.000 & & \\
\hline
\end{tabular}




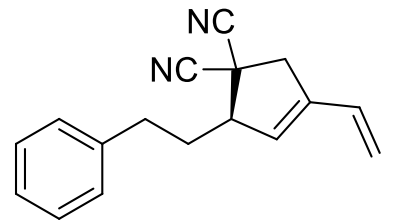

[6]

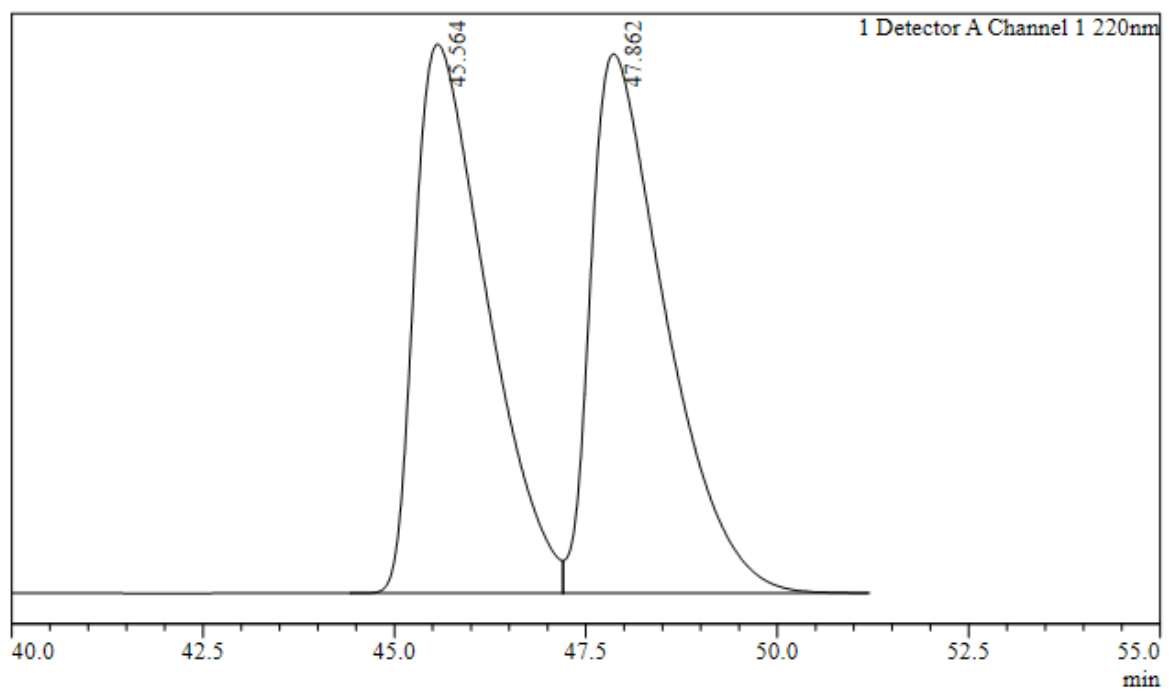

Peak Table

\begin{tabular}{|r|r|r|r|r|r|r|}
\multicolumn{2}{|c|}{ Detector A Channel 1 $220 \mathrm{~nm}$} & \multicolumn{3}{|c|}{ Peak Table } \\
Peak\# & Ret. Time & Area & Height & Area $\%$ & Conc. & Name \\
\hline 1 & 45.564 & 27416406 & 417666 & 49.538 & 49.538 & \\
\hline 2 & 47.862 & 27928078 & 410259 & 50.462 & 50.462 & \\
\hline Total & & 55344484 & 827924 & 100.000 & & \\
\hline
\end{tabular}

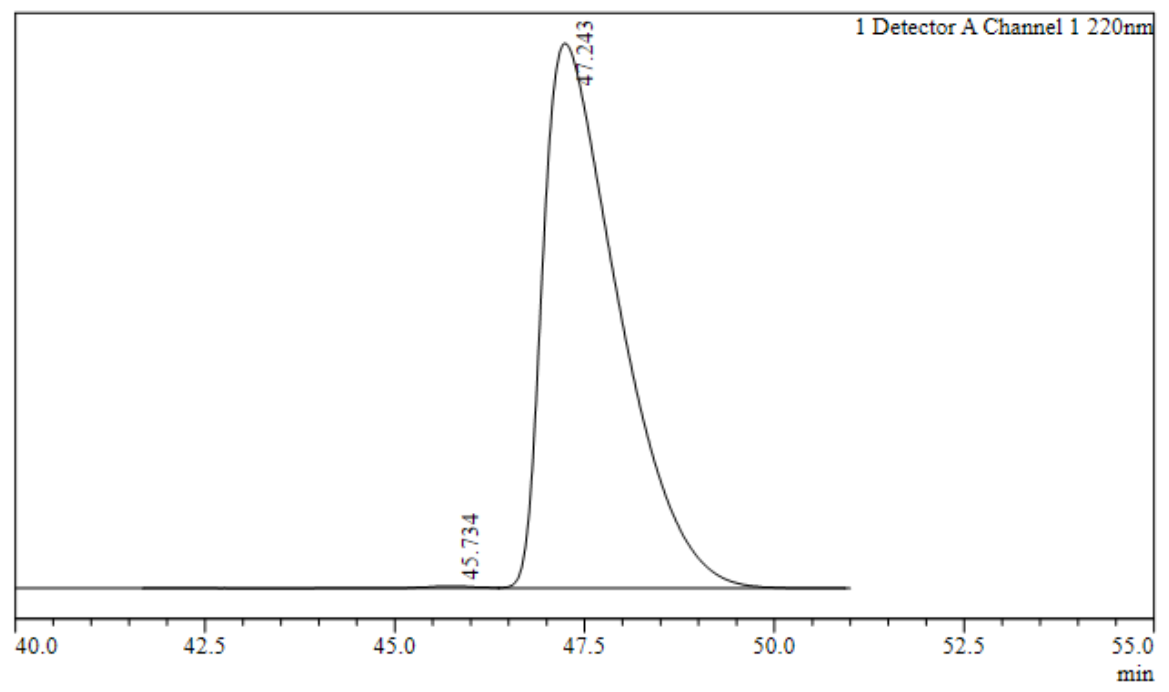

Peak Table

\begin{tabular}{|c|c|c|c|c|c|c|}
\hline \multicolumn{7}{|c|}{ or A Channel $1220 \mathrm{~nm}$} \\
\hline Peak\# & Ret. Time & $\begin{array}{l}\text { Area } \\
61625\end{array}$ & $\begin{array}{l}\text { Height } \\
1687\end{array}$ & $\begin{array}{l}\text { Area } \% \\
0.181\end{array}$ & $\begin{array}{l}\text { Conc. } \\
0.181\end{array}$ & Name \\
\hline 2 & 47.243 & 34049085 & 491251 & 99.819 & 99.819 & \\
\hline Tota & & 34110710 & 492938 & 100.000 & & \\
\hline
\end{tabular}




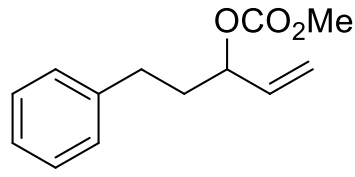

$1 a$

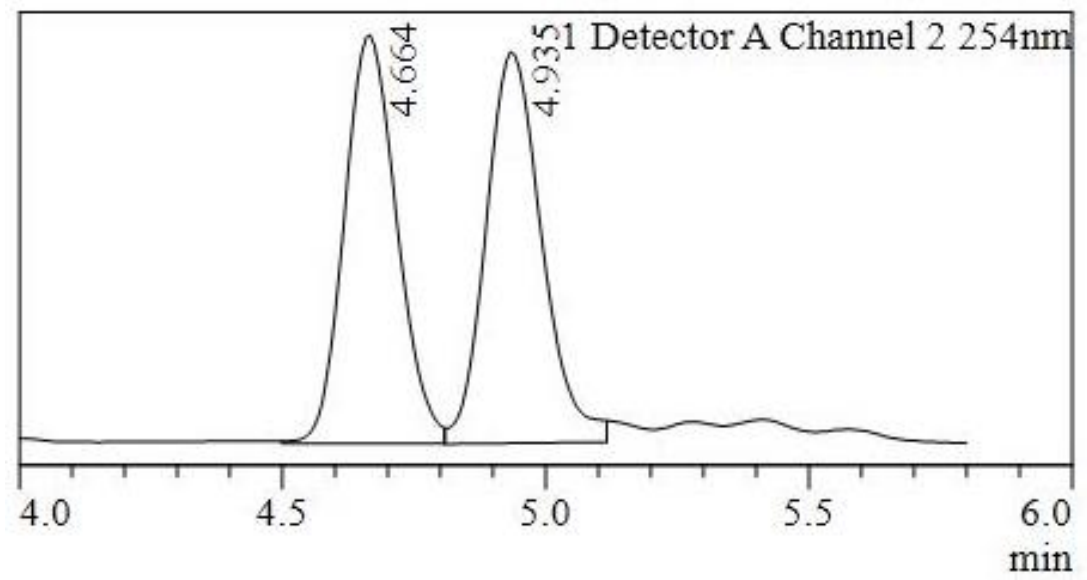

Peak Table

\begin{tabular}{|r|r|r|r|r|r|r|}
\multicolumn{8}{c|}{ Peak Table } \\
Detector A Channel 2 $254 \mathrm{~nm}$ \\
\hline Peak\# & Ret. Time & Area & Height & Area\% & Conc. & Name \\
\hline 1 & 4.664 & 62394 & 8991 & 49.549 & 49.549 & \\
\hline 2 & 4.935 & 63529 & 8603 & 50.451 & 50.451 & \\
\hline Total & & 125922 & 17595 & 100.000 & & \\
\hline
\end{tabular}
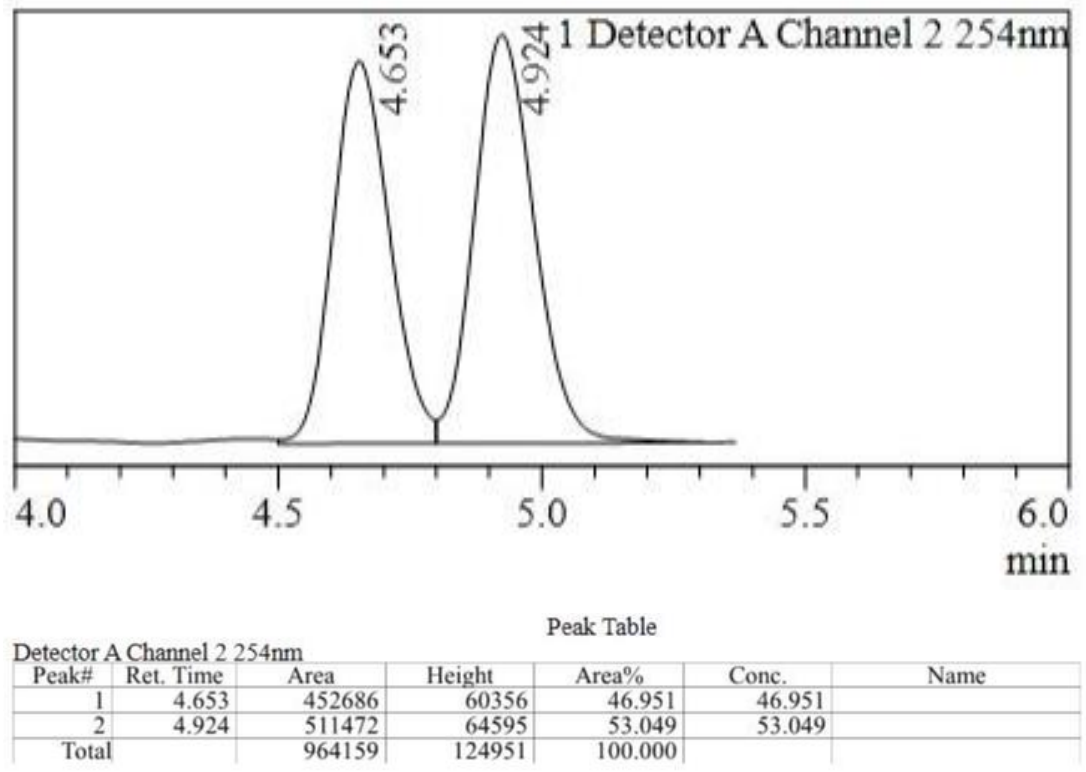


\section{References:}

1. Ghorai, S.; Chirke, S. S.; Xu, W.-B.; Chen, J.-F.; Li, C. Cobalt-Catalyzed Regio- and Enantioselective Allylic Amination. J. Am. Chem. Soc. 2019, 141, 11430-11434.

2. Takizawa, K.; Sekino, T.; Sato, S.; Yoshino, T.; Kojima, M.; Matsunaga, S. Cobalt-Catalyzed Allylic Alkylation Enabled by Organophotoredox Catalysis. Angew. Chem. Int. Ed. 2019, 58, 9199-9203.

3. Tayyari, F.; Wood, D. E.; Fanwick, P. E.; Sammelson, R. E. Monosubstituted Malononitriles: Efficient OnePot Reductive Alkylations of Malononitrile with Aromatic Aldehydes. Synthesis, 2008, 2, 279-285.

4. Ghorai, M. K.; Talukdar, R.; Tiwari, D. P. A Route to Highly Functionalized $\beta$-Enaminoesters via a Domino Ring-Opening Cyclization/Decarboxylative Tautomerization Sequence of Donor-Acceptor Cyclopropanes with Substituted Malononitriles. Org. Lett. 2014, 16, 2204-2207.

5. Grugel, C. P.; Breit, B. Rhodium-Catalyzed Asymmetric Allylation of Malononitriles as Masked Acyl Cyanide with Allenes: Efficient Access to $\beta, \gamma$-Unsaturated Carbonyls. Chem. Eur. J. 2018, 24, 15223-15226.

6. Stanley, L. M.; Bai, C.; Ueda, M.; Hartwig, J. F. Iridium-Catalyzed Kinetic Asymmetric Transformations of Racemic Allylic Benzoates. J. Am. Chem. Soc. 2010, 132, 8918-8920. 